\title{
CARDIFF
}

UNIVERSITY

PRIFYSGOL

CAERDY'

BINDING SERVICES

Tel +44 (0)29 20874949

Fax.+44 (0)29 20371921

E-Mail Bindery@Cardiff.ac.uk 



\section{A Novel Miniature Matrix Array Transducer System for Loudspeakers}

By

Razib Rashedin

A thesis submitted in partial fulfilment of the requirements for the degree of Doctor of Philosophy

$$
\text { April, } 2007
$$

Wolfson Centre for Magnetics

Cardiff School of Engineering

Cardiff University 


\section{INFORMATION TO ALL USERS}

The quality of this reproduction is dependent upon the quality of the copy submitted.

In the unlikely event that the author did not send a complete manuscript and there are missing pages, these will be noted. Also, if material had to be removed, a note will indicate the deletion.

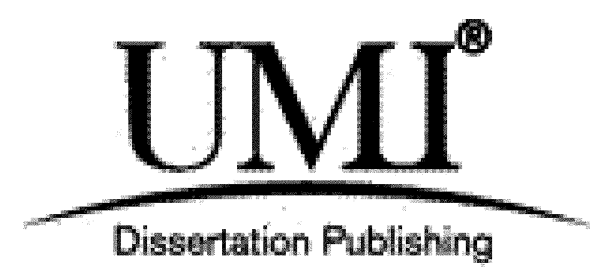

UMI U584947

Published by ProQuest LLC 2013. Copyright in the Dissertation held by the Author.

Microform Edition (c) ProQuest LLC.

All rights reserved. This work is protected against unauthorized copying under Title 17, United States Code.

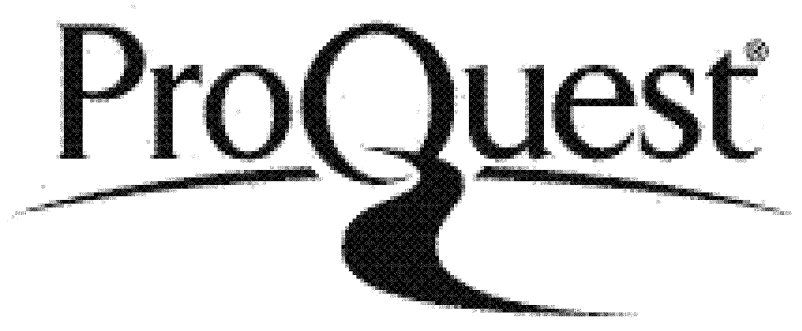

ProQuest LLC

789 East Eisenhower Parkway

P.O. Box 1346

Ann Arbor, MI 48106-1346 


\section{DECLARATION}

This work has not previously been accepted in substance for any degree and is not being concurrently submitted in candidature for any degree.

Signed.RAZIB...RASHEDIP ..........(candidate) Date..17.0.7.0.7....

\section{STATEMENT 1}

This thesis is being submitted in partial fulfilment of the requirements for the degree of $\mathrm{PhD}$

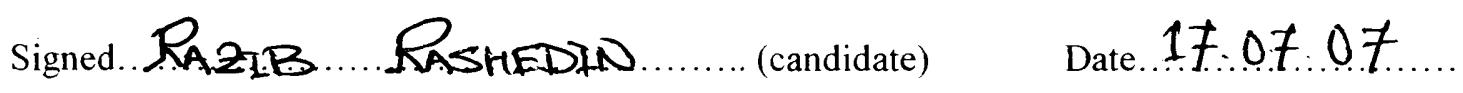

\section{STATEMENT 2}

This thesis is the result of my own investigations, except where otherwise stated.

Other sources are acknowledged by explicit references.

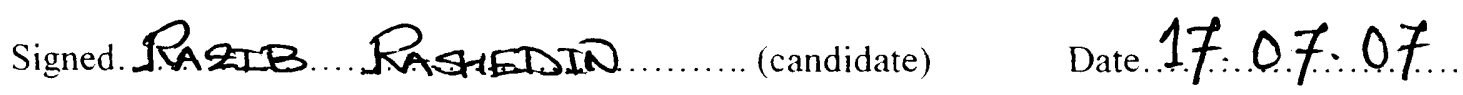

\section{STATEMENT 3}

I hereby give consent for my thesis. if accepted. to be available for photocopying and interlibrary loan, and for the title and summary to be available to outside organisation.

signed. Razib.... Rastiedir....... (candidate) Date.17.07..07

\section{STATEMENT 4-BAR ON ACCESS APPROVED}

I hereby give consent for my thesis. if accepted. to be available for photocopying and for inter-library loans after expiry of a bar on access approved by the Graduate Development Committee 
To my parents and my wife 


\section{ACKNOWLEDGEMENTS}

The work was carried out at Wolfson Centre for Magnetics, Cardiff School of Engineering, Cardiff University. First of all, I wish to express special thanks to Dr. T. Meydan who supervised this research project and gave advice and guidance that significantly contributed to the realisation of this work.

I would like to thank Prof. David Jiles and Dr. F. Borza for their valued support to this research project.

Many thanks are due to Eur Phys Paul Bartlett who devoted a good portion of his busy schedule to assist me in this work. He has answered many questions I have had and given encouragement throughout. Also thanks are offered to the academic members of Wolfson Centre, especially Dr F. I. Al Naemi for his helps throughout this project.

I should also like to thank my friends and colleagues in the Wolfson Centre for Magnetics and in the Biomedical Sciences Library for their help, support and understanding during this project work.

Finally, my greatest thanks go to my loving wife Rose who has been supportive of me throughout the project. I would like to thank her especially for her understanding and patience during all the time of my studies. 


\section{SUMMARY}

Conventional pistonic loudspeakers, by employing whole-body vibration of the diaphragm, can reproduce good quality sound at the low end of the audio spectrum. Flat panel speakers, on the other hand, are better at high frequency operation as the reproduced sound at high frequency from a flat panel speaker is not omni-directional as in the case of a conventional loudspeaker. Although flat-panel speakers are compact, small and have a better high frequency response; the poor reproduction of bass sound limits its performance severely. In addition, the flat panel speakers have a poor impulse response. The reason for such poor bass and impulse response is that, unlike the whole body movement of a conventional loudspeaker diaphragm, different parts of the panel in a flat panel loudspeaker vibrates independently.

A novel loudspeaker has been successfully designed, developed and operated using miniature electromagnetic transducers in a matrix array configuration. In this device, the whole body vibration of the panel reduces the poor bass and impulse response associated with present flat panel speakers. The multi-actuator approach combines the advantages of conventional whole body motion with that of modern flat panel speakers.

An innovative miniature electromagnetic transducer for the proposed loudspeaker has been designed, modelled and built for analysis. Frequency Responses show that this novel transducer is suitable for loudspeaker application because of its steady and consistent output over the whole audible frequency range and for various excitation currents. Measurements on various device configurations of this novel miniature electromagnetic transducer show that a moving coil transducer configuration having a magnetic diaphragm is best suited for loudspeaker applications. Finite element modeling has been used to examine single transducer operation and the magnetic interaction between neighbouring transducers in a matrix array format. Experimental results show the correct positioning of the transducers in a matrix configuration reduces the effects of interferences on the magnetic transducers. In addition, experimental results from the pressure response measurement show an improvement in bass response for the longer array speaker. 


\section{CONTENTS}

\section{ACKNOWLEDGEMENTS}

\section{SUMMARY}

CHAPTER 1 Aims of the Investigation.

\section{CHAPTER 2 Loudspeakers}

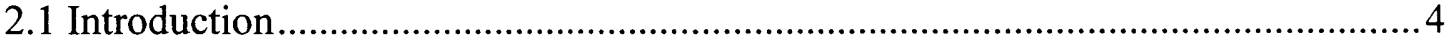

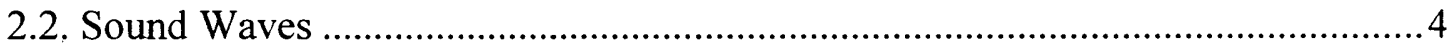

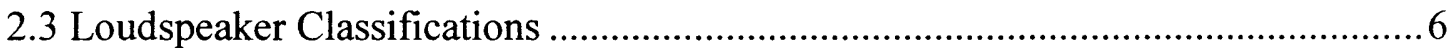

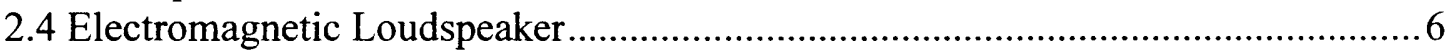

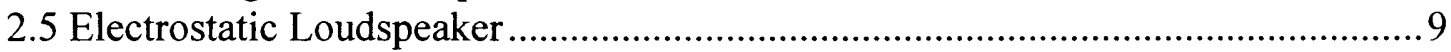

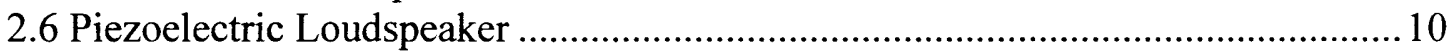

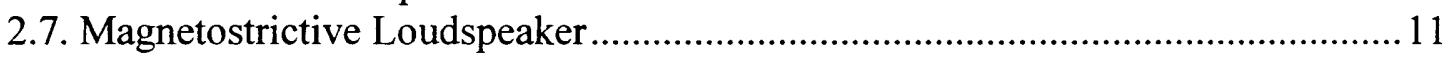

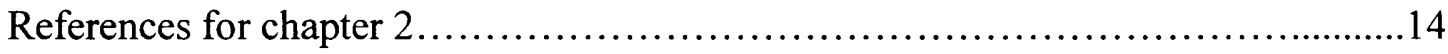

CHAPTER 3 Evolution of Loudspeakers

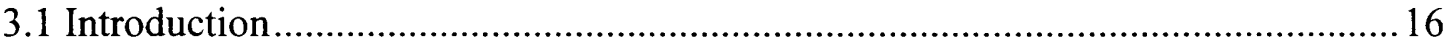

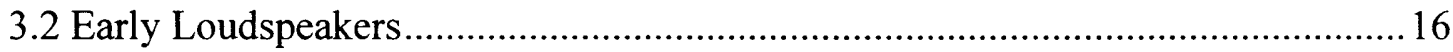

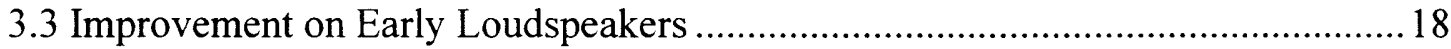

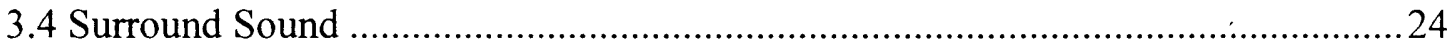

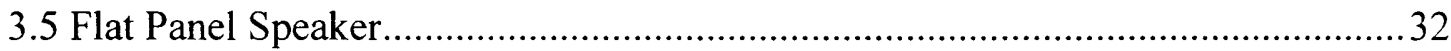

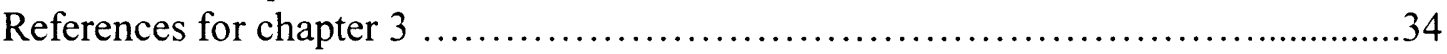

\section{CHAPTER 4 Conventional and Flat Panel Loudspeaker}

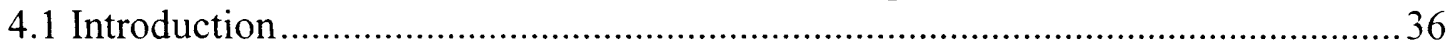

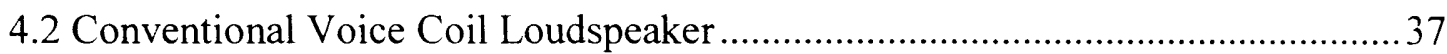

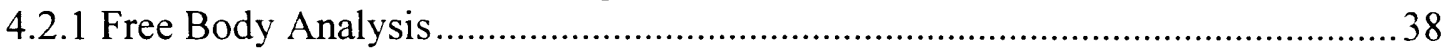

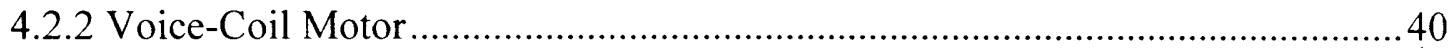

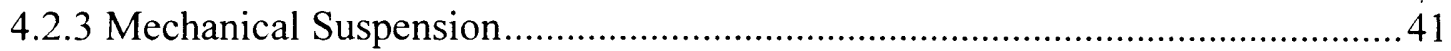

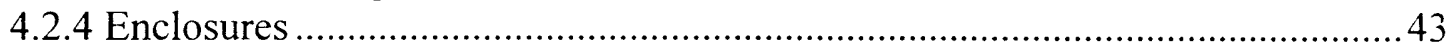

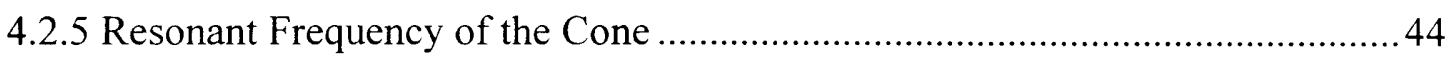

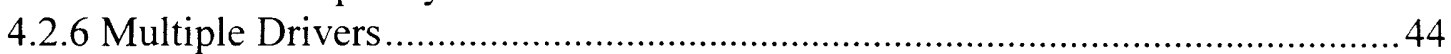

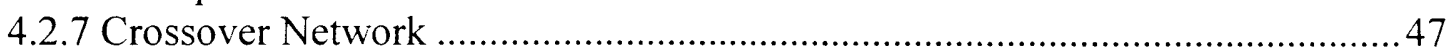

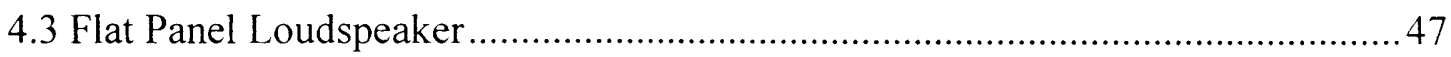

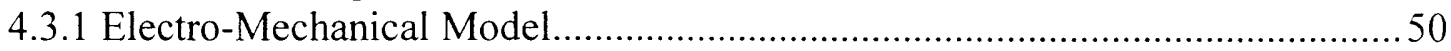

4.3.2 Advantages of Distributed-Mode Technology ................................................51

4.3.3 Disadvantages of Distributed-Mode Technology ............................................53

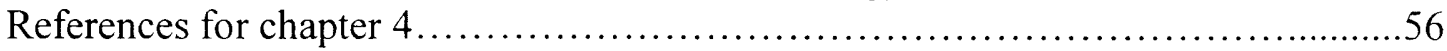

CHAPTER 5 Previous Research on Loudspeakers

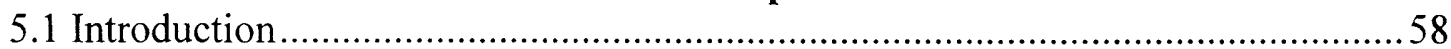

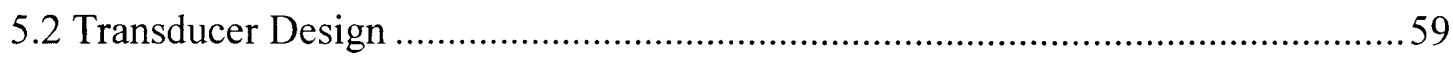

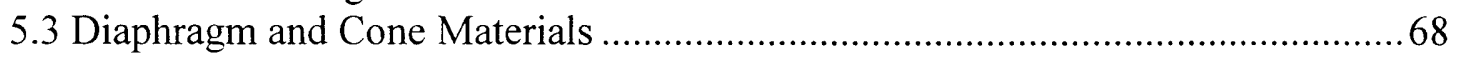




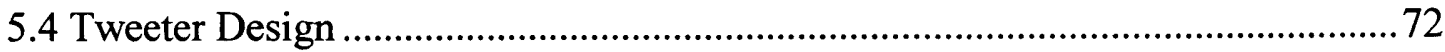

5.5 Distortion Analysis and Reduction ...................................................................73

5.6 Measurement..............................................................................................

5.7 Flat Panel Loudspeaker........................................................................................ 84

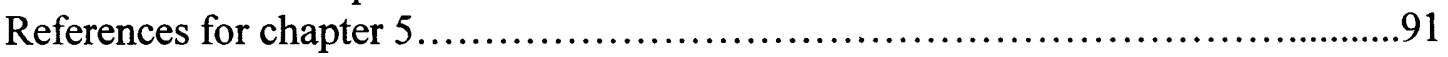

\section{CHAPTER 6 Design and Development}

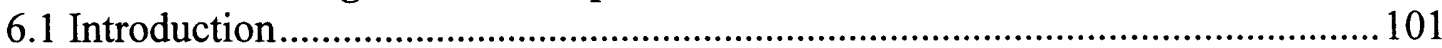

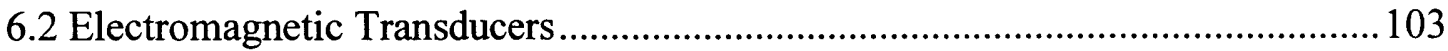

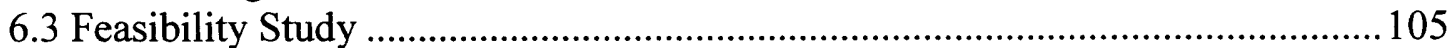

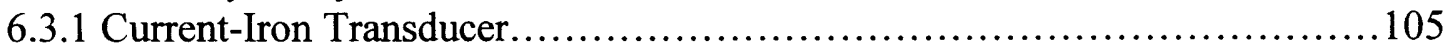

6.3.1.1 Experiment on a Commercial Solenoid Actuator............................106

6.3.1.2 FEM Simulation on Solenoid Actuator......................................110

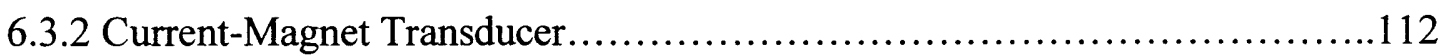

6.3.2.1 Experiment on a Commercial Voice Coil Actuator............................113

6.4 Design of the Novel Transducer Speaker......................................115

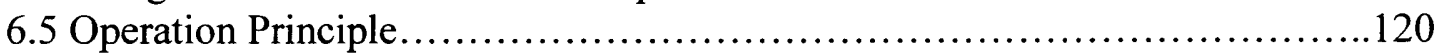

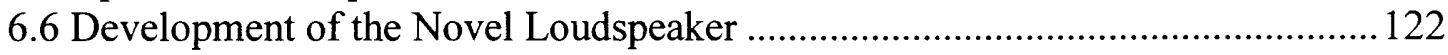

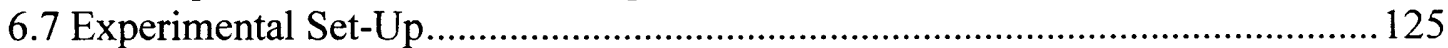

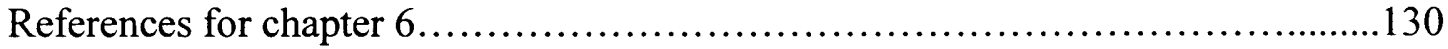

\section{CHAPTER 7 Experimental Results and Analysis}

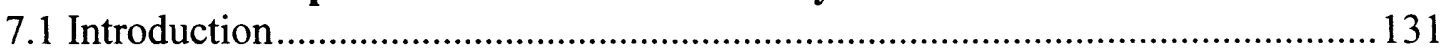

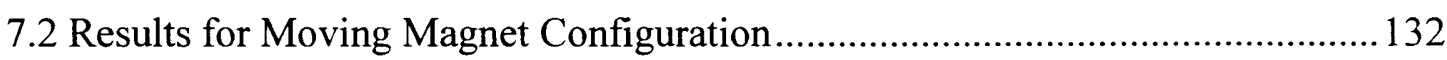

7.2.1 Displacement versus Frequency Responses...................................................132

7.2.2 Displacement versus Current Responses ......................................................135

7.2.3 Acoustic Intensity versus Frequency Response ………...................................138

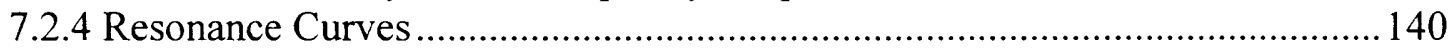

7.2.5 Acoustic Intensity versus Current Response.................................................... 142

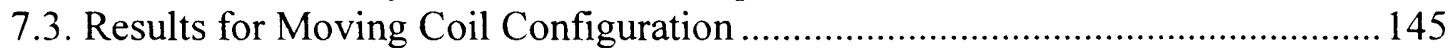

7.3.1 Displacement versus Frequency Responses................................................... 145

7.3.2 Displacement versus Current Responses .......................................................147

7.3.3 Acoustic Intensity versus Frequency Response ................................................150

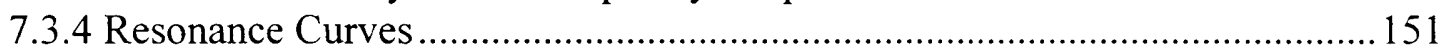

7.3.5 Acoustic Intensity versus Current Response.....................................................152

7.4 Results for Moving Magnet with a Non-Magnetic Bottom Layer........................155

7.4.1 Displacement versus Frequency Responses.....................................................156

7.4.2 Displacement versus Current Responses ....................................................... 158

7.4.3 Acoustic Intensity versus Frequency Response ................................................. 161

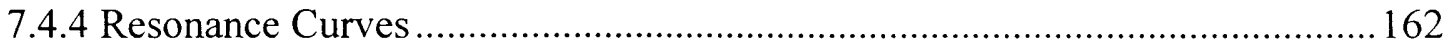

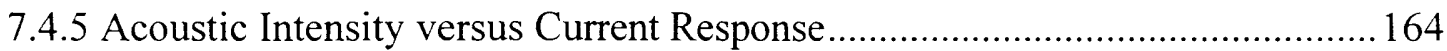

7.5 Results for Moving Coil with a Non-Magnetic Diaphragm .................................167

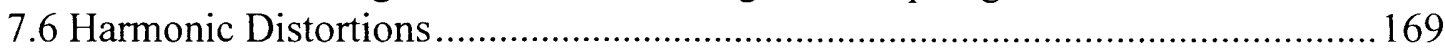

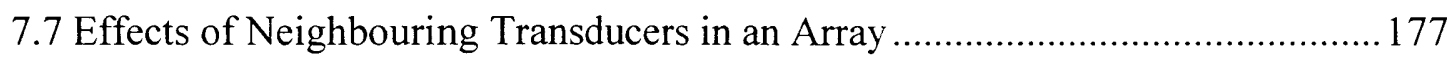

7.8 Displacement Profile of the Vibrating Panel ..................................................... 180

7.9 Pressure Response Curves from Transducers Arrays ………………................. 182

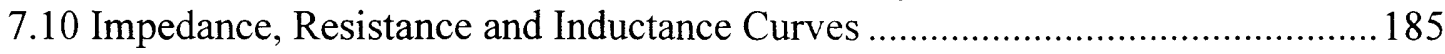

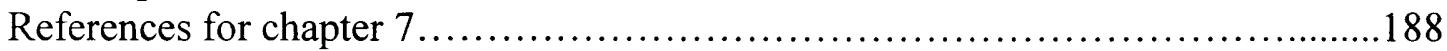


CHAPTER 8 Discussion

CHAPTER 9 Conclusion and Future Work

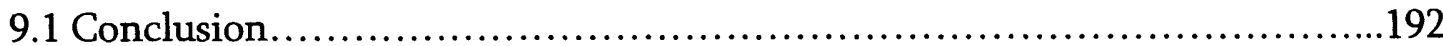

9.2 Future Work ................................................... 193

Appendix A: List of Publications

Appendix B: Publications 
For decades, the design concept of conventional loudspeaker has been centred on the principle of rigid piston. The common practice is to make the diaphragm of the loudspeaker as light and stiff as possible such that the loudspeaker behaves as a rigid piston. Furthermore, the surface is generally made conical to further increase rigidity as well as on-axis sensitivity at low frequency. Although the technology is well established, conventional loudspeakers suffer from a problem: the sound generated by conventional loudspeakers becomes increasingly directional for high frequencies. This "beaming" effect results in the drop of sound power at the high frequency region. Consequently, an audio system generally requires crossover circuits and multi-way loudspeakers to cover the audible frequency range, which makes the entire system unnecessarily large.

On the other hand, flat panel speakers are based on a philosophy contradicting conventional design. A panel loudspeaker primarily consists of a panel and an exciter which is essentially a voice-coil driver with the coil attached to the panel. In lieu of a rigid diaphragm as used in conventional loudspeakers, flexible panels are employed as the primary sound radiators. Resonance of flexural motion is encouraged such that the panel vibrates as randomly as possible. The sound field produced by this type of distributed mode loudspeaker (DML) is very diffuse at high frequency. As claimed by the supporters of panel speakers, DML provides advantages over the conventional counterpart such as compactness, linear on-axis, attenuation, insensitivity to room conditions, bi-polar radiation, good linearity, and so forth. Of particular interest is that the DML has a less pronounced beaming problem at high frequencies than conventional loudspeakers, which bypass the need for crossover circuits and multiway high frequency speakers. However, although the flat panel speaker technology has a number of advantages over conventional loudspeakers, it also suffers from a problem: the poor reproduction of bass sound limits its performance severely. Additionally, the flat-panel speakers have a poor impulse response. The reason for such poor bass and impulse response is that, unlike the whole-body movement of a 
conventional loudspeaker diaphragm, different parts of the panel in a flat-panel loudspeaker vibrate independently.

The aim of this project is therefore to introduce a novel loudspeaker design that will allow a flat panel loudspeaker to have the benefits of a conventional speaker technology without losing its own advantageous characteristics. Therefore, the new loudspeaker design aims to introduce whole-body motion in flat-panel speakers. Instead of a single exciter, the proposed miniature matrix array transducer system for the loudspeaker will employ numerous miniature transducers to vibrate coherently and produce sound effectively at the low and high range of the audio spectrum. The multi-actuator approach combines the advantages of conventional whole-body motion with that of modern flat-panel speakers. This new loudspeaker design will also provide improvement in impulse response.

The study comprises two main parts involving modelling, theory and feasibility analysis for the first part, and experimental analysis of the novel transducer speaker for the second one. The theory and the feasibility part of the project looks at the possibility of employing various forms of electromagnetic transduction mechanisms for the new loudspeaker design. Both the current-iron and a current-magnet combination of the electromagnetic transducers have been explored in this thesis. This part leads to understand how actuation behaviour and frequency response vary according to the magnetic properties of the surrounding material, the effects of eddy currents at high frequency and other factors that affect electromagnetic transduction mechanisms. After the feasibility analysis, this project concentrates on the design and development of a novel electromagnetic transducer speaker that can introduce pistonic motion in a flat panel speaker.

The thesis looks at the various possible configurations of the new transducer speaker in order to optimise the performance of the novel loudspeaker. The various configurations of the transducer speaker have been built and tested to observe the frequency response, linearity of the displacement curves, acoustic intensity, impedance characteristics and harmonic distortions. The observance of the performance criteria of the various device configurations using experimentation and modelling enables the choice of the most suitable transducer design for the miniature 
matrix array loudspeaker. This study also looks at the performance of the matrix array transducer speaker by experimenting and comparing two different configuration of the matrix array loudspeaker.

The development of a novel miniature transducer speaker utilizing electromagnetic transduction mechanism aims to introduce a new flat panel speaker technology that can overcome the limitations of the past loudspeaker designs and can provide a better and enhanced performance in the audio frequency spectrum. 


\subsection{Introduction}

The loudspeaker is one of a class of electroacoustical transducers that convert electrical signal to mechanical pressure waves. The loudspeaker is actuated by electrical signals to produce acoustical energy through the mechanical vibrations of a radiating element [1]. The waves, which lie within the audible frequency range which is approximately $20 \mathrm{~Hz}$ to $20 \mathrm{kHz}$, are perceived as sound.

The first patent on a loudspeaker was issued to Siemens in 1877 [2]. It was intended to be used in telephone equipment, and as outlined in the original patent application, bears a striking resemblance to present day units. The modern inertia controlled speaker was first described by Rice and Kellogg in 1925 [3].

\subsection{Sound Waves}

Sound is a form of wave motion and is created only when something moves or vibrates. The vibration of an object disturbs the air, the resulting air disturbance enters the air, and if the disturbance in air is in the audible frequency range, it agitates the ear drum, the auditory nerve is excited, and we experience the sensation of sound. Sound waves are known as longitudinal waves, since the vibration of the air particles takes place along the direction of travel of the wave.

In order that a body or medium vibrate it must possess two properties: (1) Inertia or Mass; (2) Elasticity, i.e. power to resist change of size or shape and to recover its original condition when disturbed [4]. Since air has mass and is also an elastic medium, the vibration of a loudspeaker diaphragm results in the vibration of air. The frequency of the air vibration matches the frequency of the vibrations of the source. 
The sound waves generated by the vibration of a loudspeaker diaphragm consist of a succession of pulses of compressed air or compressions, separated by regions of rarefied air or rarefactions (Fig. 2.1) [5].

When the diaphragm of the loudspeaker moves outwards, it compresses the air and this compression travels outwards. When the diaphragm moves inwards the air near to them moves back again, causing a rarefaction. All the air particles move back in turn, with the result that the rarefaction travels outwards. Again, the diaphragm moves outwards, and a second compression is sent out, and so on.

The distance between the centres of two adjacent compressions or rarefactions is called the wavelength of the sound. The sound wave travels a distance equal to the wavelength while the diaphragm makes one complete vibration.

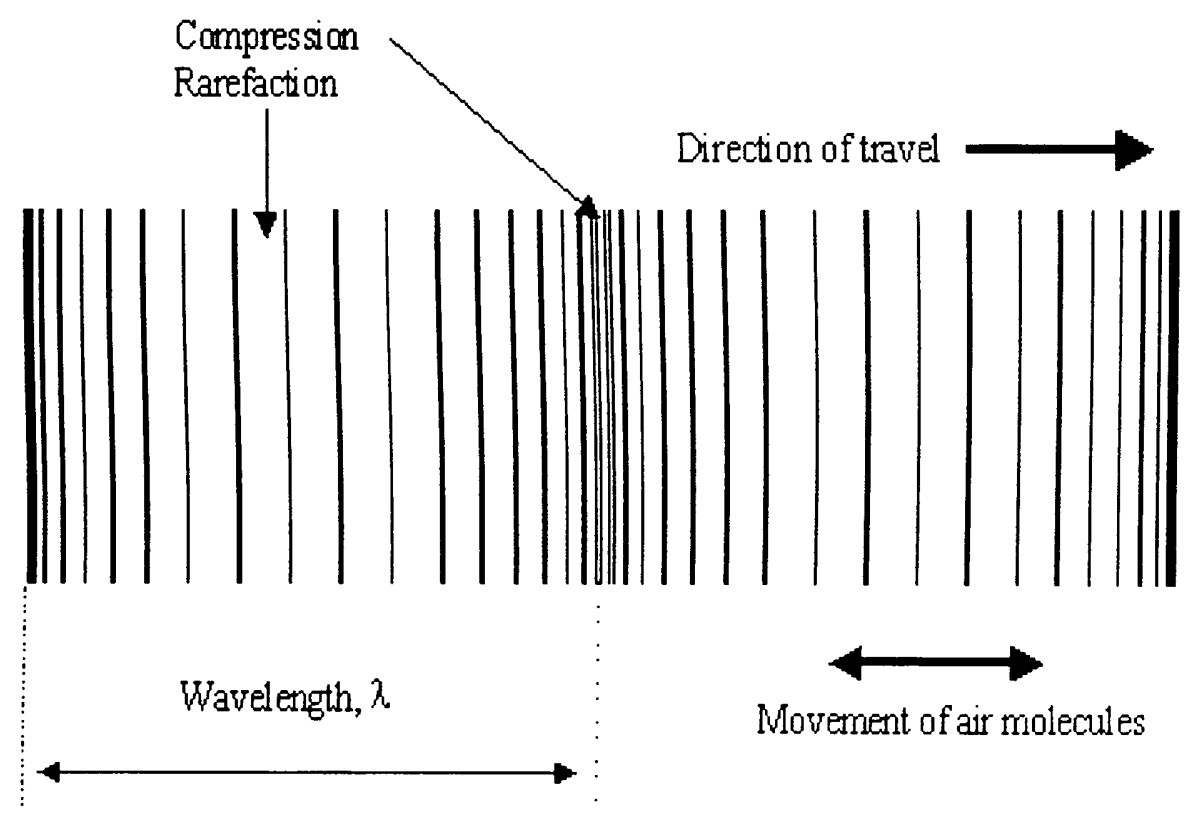

Figure 2.1: Sound Waves [5] 


\subsection{Loudspeaker Classifications}

The classification of loudspeakers is based on the transduction mechanism and the transducer itself. Sound is mainly produced by loudspeakers having the following mechanisms of transduction:

(1) Electro-magnetic

(2) Electrostatic

(3) Piezoelectric

(4) Magnetostrictive

Every speaker consists of two elements, the motor element that converts the electrical signal into a mechanical force, and the acoustic radiator that matches the mechanical output to the acoustic medium. The first is the interface between electrical and mechanical systems, the second between the mechanical system and the medium. The four different transduction mechanisms mentioned above will be discussed in detail in the following sections.

\subsection{Electromagnetic Loudspeaker}

Electromagnetic loudspeakers have been in use for several decades. They were originally invented by Kellogg and Rice (circa 1920) [1]. A cutaway view of an electromagnetic loudspeaker driver typical of modern designs is shown in figure 2.2 [6] and a cross-sectional diagram is shown in figure 2.3 [6]. The behaviour of a driver is governed by basic principles of physics. An alternating current is supplied to the leads of the driver. These leads are connected to a wire that wraps around a coil former, creating what is known as the voice coil. The coil has an electrical resistance and inductance associated with it. It is positioned within the gap created between a hollow cylindrical magnet (e.g., North Pole) and a solid cylindrical pole piece (e.g., South Pole). The latter is located within the hollow coil former. Current applied to the voice coil flows in a circular direction around the windings. The magnet structure provides magnetic flux through the coil with field lines running perpendicular to the direction of current flow. 
As is well known in the study of electromagnetism, if a current flows in the presence of a magnetic field, a Lorentz force is created. When applied to the geometry of a moving-coil loudspeaker driver, the orthogonally oriented Lorentz Force simplifies to the product of the effective magnetic flux density, the effective length of the coil in the field, and the current flowing in the coil. Since the applied current alternates, the Lorentz force likewise alternates, causing the voice coil (and anything attached to it) to oscillate in an analogous manner. The voice coil is attached to the former, which is attached to a cone or diaphragm. This diaphragm assembly is held in place by a suspension system that centres the voice coil in the magnet gap. Suspension systems typically consist of two separate flexible components: the surround and the spider. These spaced components serve to constrain the cone vibrations to motion along a single axis and supply a restoring force to return the cone to its rest position. The suspension system has a compliance and resistance associated with it. The cone, coil former, voice coil, parts of the suspension system and lead wires ideally move in phase as lumped elements with a certain effective mass. Oscillations of the cone produce fluctuations in air pressure that radiate away from the driver as sound waves.

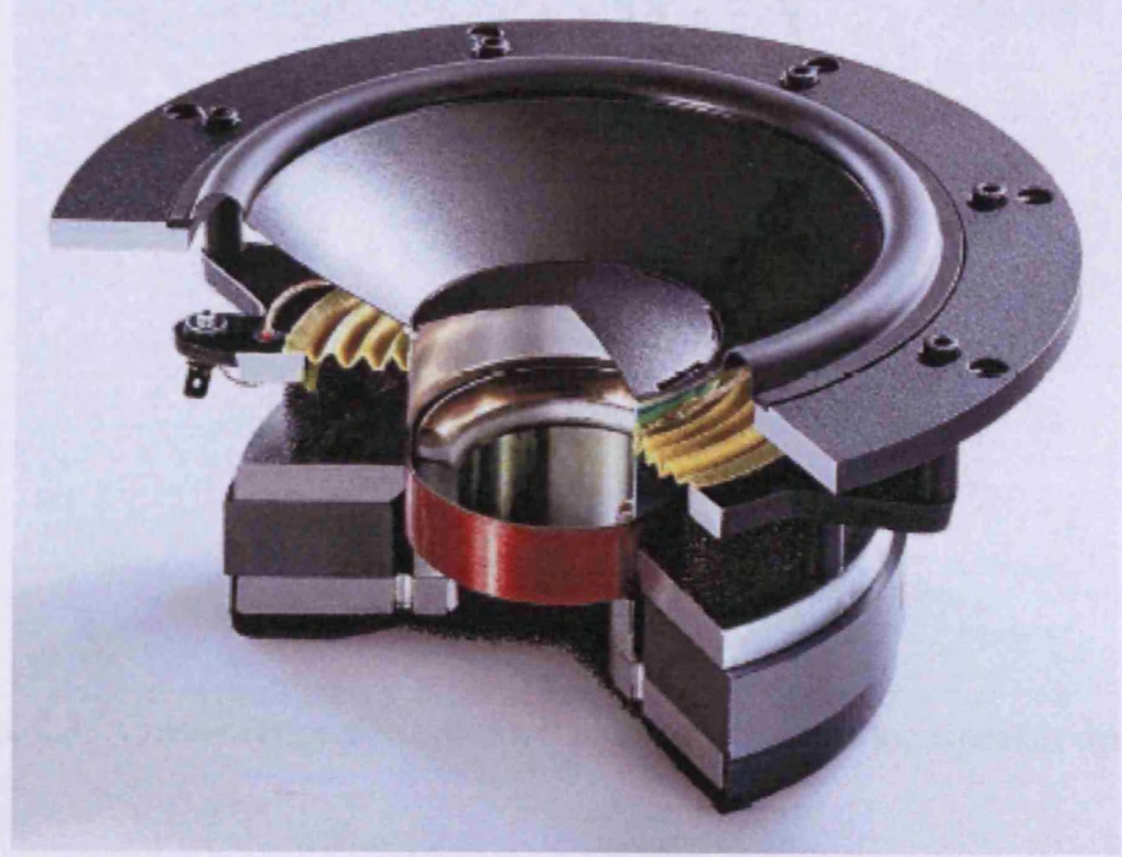

Figure 2.2: Cutaway view of a typical moving-coil loudspeaker driver [6] 


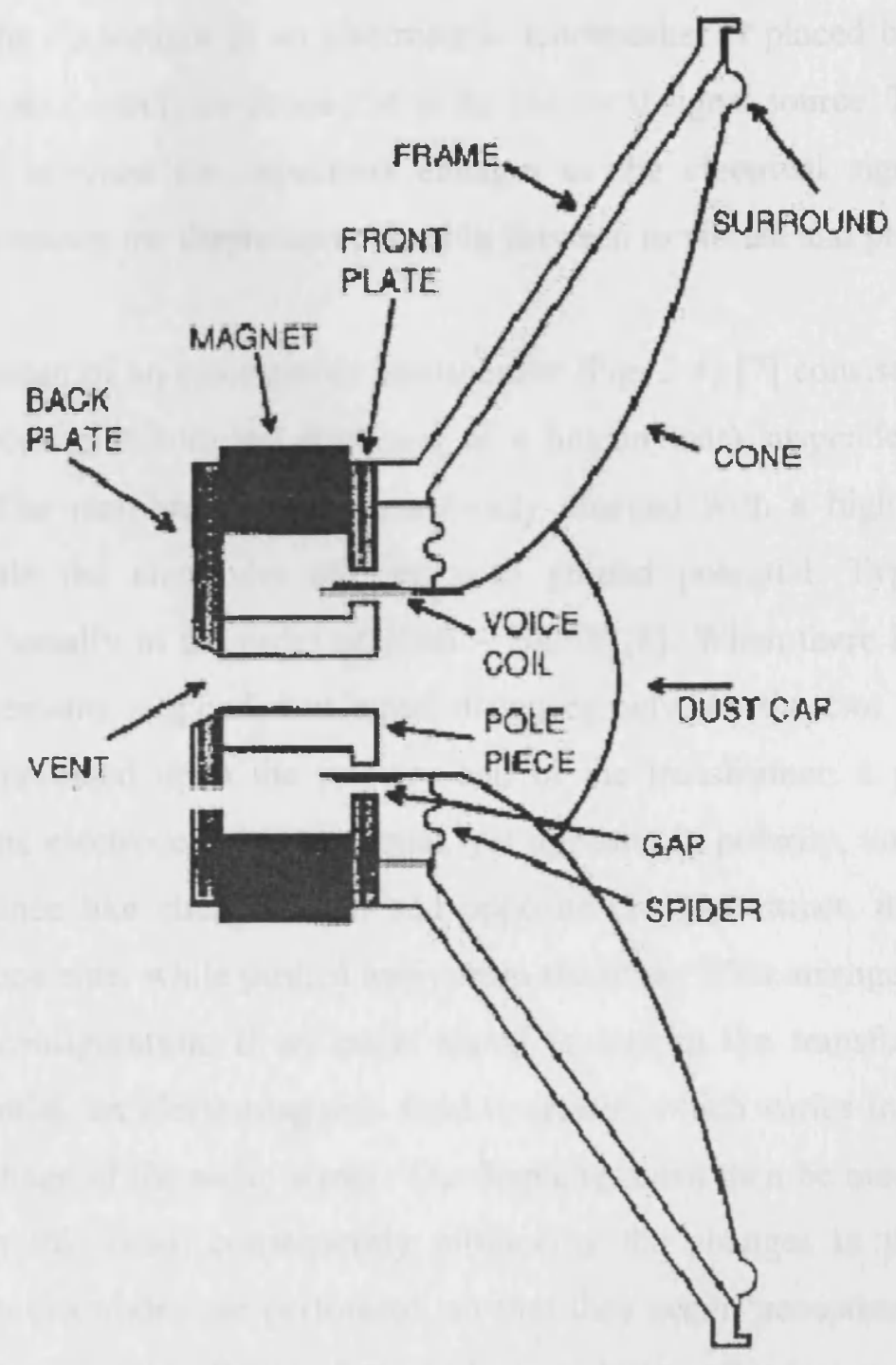

Figure 2.3: Cross-sectional view of a typical moving-coil loudspeaker driver [6] 


\subsection{Electrostatic Loudspeaker}

The operation of an electrostatic loudspeaker is based on the principle of electrostatic induction. The diaphragm in an electrostatic loudspeaker is placed between the two conductive plates which are connected to the electrical signal source. The electrostatic field pattern between the capacitors changes as the electrical signal changes its polarity; this causes the diaphragm placed in between to vibrate and produce sound.

The basic design of an electrostatic loudspeaker (Fig. 2.4) [7] consists of a very thin plastic membrane (1/10th the thickness of a human hair) suspended between two electrodes. The membrane is electrostatically charged with a high DC polarizing voltage, while the electrodes are fed with ground potential. Typical polarizing voltages are usually in the order of $2000-3000 \mathrm{~V}$ [8]. When there is no signal, the diaphragm remains suspended at equal distances between the two electrodes. If a voltage is impressed upon the primary coil of the transformer, a positive voltage appears at one electrode, while an equal, yet opposite in polarity, voltage appears at the other. Since like charges repel and opposite charges attract, the diaphragm is attracted to one side, while pushed away from the other. This arrangement is called a 'push-pull' configuration. If an audio signal is sent to the transformer instead of ground potential, an electromagnetic field is created which varies in response to the changing voltage of the audio signal. The diaphragm can then be made to move back and forth in this field, consequently mimicking the changes in the input signal. Finally, both electrodes are perforated, so that they seem 'acoustically transparent,' thus avoiding pressure effects of trapped air and also allowing acoustic energy to move away from the diaphragm [8].

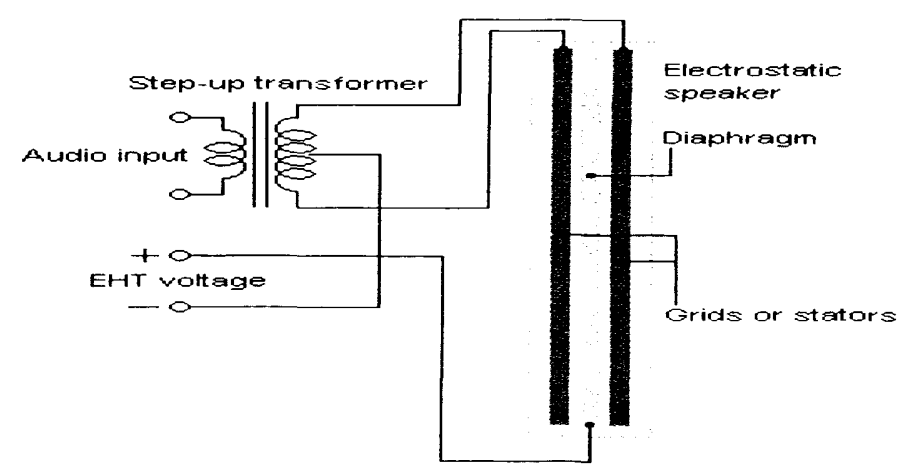

Figure 2.4: Schematic of an electrostatic speaker set-up [7] 
Two methods of constructing electrostatic speakers have emerged. The first involves stretching the diaphragm over a frame, supporting it at its edges, and leaving the middle unattached and free to vibrate. The second method, which is much less common today than it was in the 1950's and 60's, uses an "inert diaphragm" supported by several tiny elements equally spaced across its surface. These spacers hold the diaphragm in the centre between the electrodes, yet more importantly allow the diaphragm to be curved without seriously impeding its ability to vibrate [8]. This capability of curving the diaphragm is an important tool in controlling the directionality of radiated sound.

\subsection{Piezoelectric Loudspeaker}

The operation principle of piezoelectric loudspeakers is based on the property of piezoelectric materials. Piezoelectric materials are materials that expand/contract when an electric field is applied to them (Fig. 2.5) [9]. They also will produce an electric field across themselves if a mechanical force is applied to them. This exclusive property of the piezoelectric materials has been used for the actuation purpose in loudspeakers. The deformation of the material under A.C. signal causes vibration in air and hence produces sound.

The piezoelectric effect happens in materials with an asymmetric crystal structure. When an external force is applied, the charge centres of the crystal structure separate creating electric charges on the surface of the crystal. This process is also reversible. Electric charges on the crystal cause a mechanical deformation in the piezoelectric material. Quartz, Turmalin, and Seignette are common natural piezoelectrics [10].

Piezoelectrics deform linearly with applied electric field. Conventional piezoelectric materials only deform up to $0.1 \%[10]$ and therefore to create significant deformation in a piezoelectric material, a very high voltage is required. 


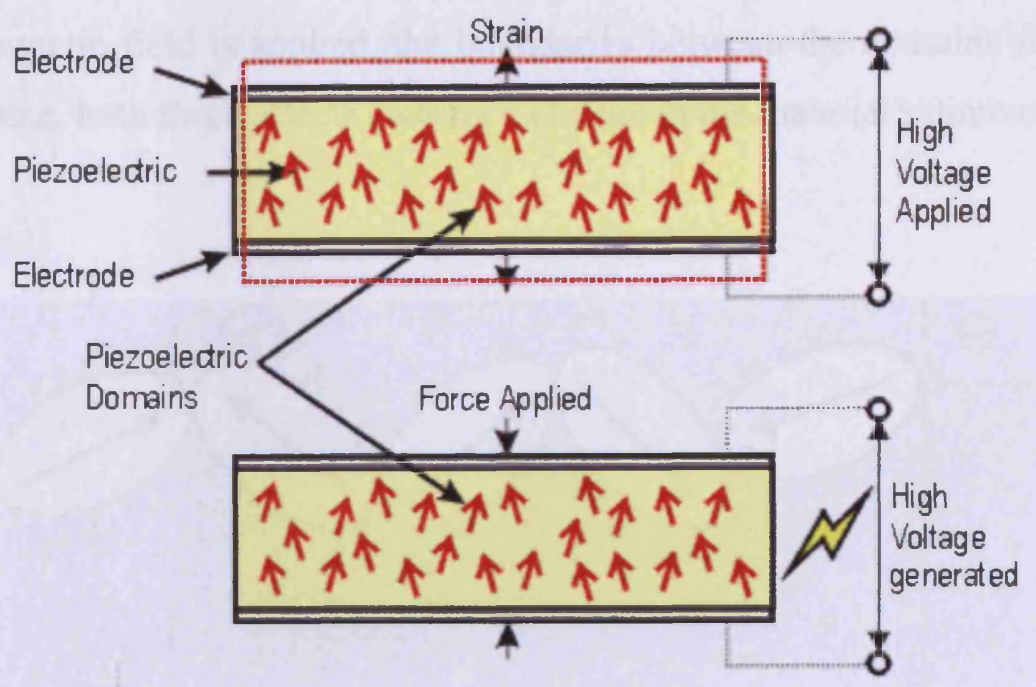

Figure 2.5: Schematic diagram of the property of a piezoelectric material [9]

\subsection{Magnetostrictive Loudspeaker}

When some materials are placed in a magnetic field (which alters the materials magnetic state), a change in the physical dimensions of these materials occurs. This effect is called magnetostriction (Fig. 2.6) [11], and this phenomenon have been utilised to build magnetostrictive loudspeaker because it represents an avenue for converting magnetic energy into physical motion for transducer applications.

Magnetostriction occurs in the most ferromagnetic materials and leads to many effects $[12,13]$. The most useful one to refer to is the Joule effect. The Joule effect states 'the Magnetostriction of a Magnetostrictive material is proportional to the magnitude of an applied field' [14]. It was discovered by James Joule in 1842 when he noticed a change in length of a piece of nickel when it was magnetised. The Joule effect is responsible for the expansion (positive magnetostriction) or the contraction (negative) of a rod subjected to a longitudinal static magnetic field. In a given material this magnetostrain is quadratic and occurs always in the same direction whatever is the fields' direction. Internally, ferromagnetic materials have a crystal structure that is divided into domains, each of which is a region of uniform magnetic polarisation. 
When a magnetic field is applied, the boundaries between the domains shift and the domains rotate, both these effects causing a change in the material's dimensions.

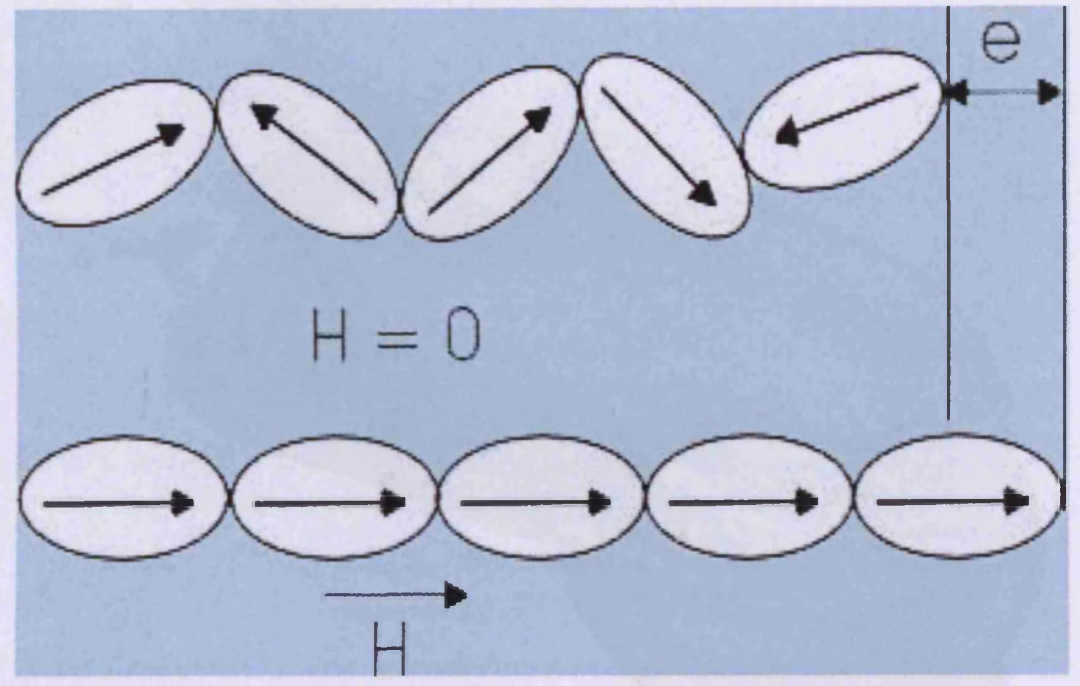

Figure 2.6: The Magnetostriction phenomena [11]

Iron, nickel and cobalt and also alloys of these materials are the simplest form of magnetostrictive materials. Recently discovered rare earth-iron "giant" magnetostrictive materials (GMM) feature magnetostrains which are two orders of magnitude larger than nickel. Among them, Terfenol-D presents the best compromise between a large magnetostrain and a low magnetic field. The name Terfenol-D comes from TERbium, FE (Iron) and Dysprosium, the three metals used in its construction. NOL stands for Naval Ordiance Labs where the material was originally created for use in high quality sonar devices for use in naval submarines. The linearity in a Terfenol-D material is obtained by applying a magnetic bias and a mechanical prestress in the active material [14].

A magnetostrictive loudspeaker (Fig. 2.7) [15] can be developed using the giant magnetostrictive property of the Terfenol-D material. In a magnetostrictive loudspeaker, Terfenol-D is placed within an aluminium case, around which is wrapped a coil. . When activated by a magnetic field, the Terfenol-D expands and contracts at very high frequency and with dramatic force. The magnetostrictive 
loudspeaker harnesses this force and transfers it to the surface to which it is attached, creating vibrations and effectively turning that surface into a sounding board.

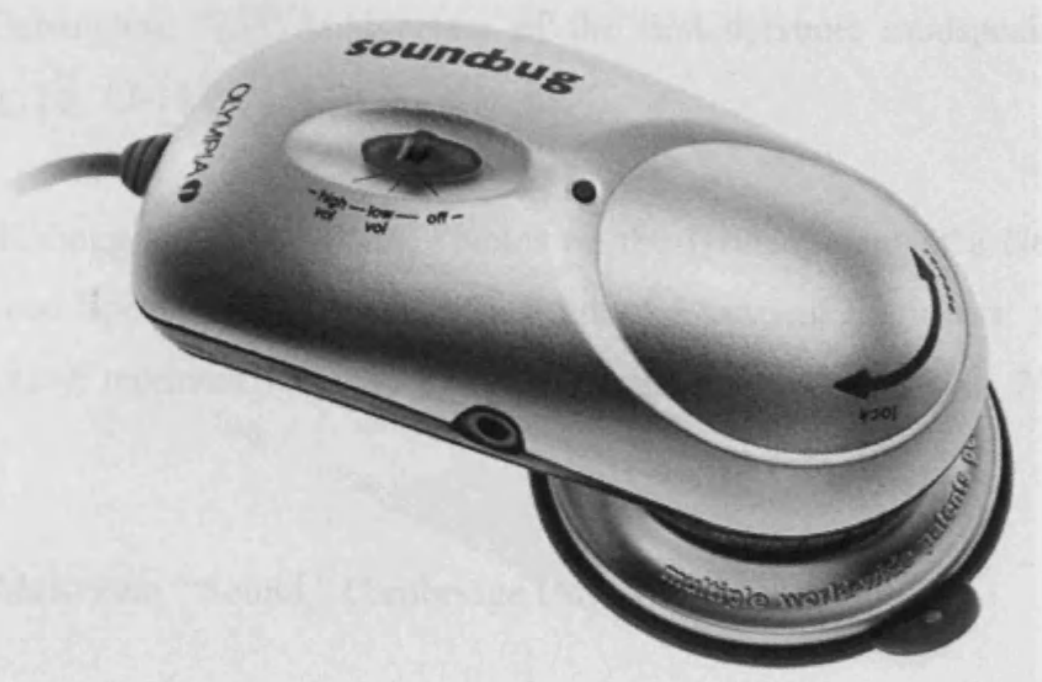

Figure 2.7: Soundbug developed by Newland Scientific uses magnetostriction to produce sound [15] 


\section{References:}

[1] J. Chernof, "Principles of loudspeaker design and operation," IRE Transactions on Audio, vol. 5, Issue 5, pp. 117-127 (1957).

[2] M.S. Corrington, " $75^{\text {th }}$ Anniversary of the first dynamic loudspeaker," Audio Engineering, pp. 12-13 (1953).

[3] E. W. Kellogg and C. W. Rice, "Notes on the Development of a New Type of Hornless Loud Speaker," J. American Institute of Electrical Engineers, vol. 44, pp. 982-991 (1925); reprinted J. Audio Engineering Society, vol. 30, Issue 7/8, pp. 512521 (1982).

[4] A.E.E. McKenzie, "Sound," Cambridge University Press, 1948

[5] Image of Sound Waves -

http://ww.antonine-education.co.uk/Physics A2/Module 4/Topic 4/wav 9.gif

[6] B.E. Anderson, "Derivation of Moving-Coil Loudspeaker Parameters using Plane Wave Tube Techniques," M.S. Thesis, Brigham Young University, Chap. 1 (2003).

[7] Image of Electrostatic Speaker Set-up -

http://en.wikipedia.org/wiki/Image:Es_spk.gif

[8] P. J. Baxandall, "Electrostatic Loudspeakers," Loudspeaker and Headphone Handbook. Reed Educational and Professional Publishing Ltd, 1998

[9] Image of Piezoelectric Material http:/wwwazom.com/work W3RUE3K9c3NUAjqa9j78 files/image002.gif

[10] J. C. Tucker, "Piezoelectric Linear Actuators," in Actuation for Mobile MicroRobotics, North Carolina State University Website 
[11] Image of Magnetostriction Phenomena -

http://www.esanet.it/chez_basilio/immagini/magnetostriction.jpg

[12] R.M. Bozorth, "Ferromagnetism," Van Nostrand, 1951

[13] E. D. Lacheisserie, "Magnetostriction: Theory and Applications," CRC Press, 1993

[14] Y. Yamamoto, T. Makino, H. Matsui, "Micro Positioning and Actuation Devices Using Giant Magnetostriction Materials," Proc. IEEE Intl. conf. on Robotics and Automation, vol. 4, pp. 3635-3640 (2000)

[15] Image of SoundBug - http://www.gadgets.dk/bigfiles/Soundbug.JPG 


\subsection{Introduction}

The nature of sound was appreciated in very early times. Aristotle was satisfied that the sensation of sound is conveyed to the ear as disturbances of the air, and it had been shown that any body that was emitting sound was vibrating. The beginning of the evolution of loudspeakers stretches back to early part of the nineteenth century. The basis for the modern electrodynamic loudspeaker originally arose from the works of Oersted in connection with the discovery of the magnetic effect of an electric current in 1820 and of Arago (1820) and Davy (1821) who discovered that magnetism could be induced in a piece of iron by action of electric current.

\subsection{Early Loudspeakers}

In 1874 Ernst W. Siemens was the first to describe the "dynamic" or moving-coil transducer, with a circular coil of wire in a magnetic field and supported so that it could move axially. He obtained an U. S. patent for a "magneto-electric apparatus" for "obtaining the mechanical movement of an electrical coil from electrical currents transmitted through it"[1]. However, he did not use his device for audible transmission. as did Alexander G. Bell who patented the telephone in 1876. In 1877 [2]. Siemens invented a nonmagnetic parchment diaphragm as the sound radiator of a moving-coil transducer. The diaphragm could take the form of a cone, with an exponentially flaring "morning glory" trumpet form. This was the first patent for the loudspeaker horn that would be used on most phonographs players in the acoustic era [1].

In 1915. the foundations for the first commercial good-sounding loudspeaker were laid by Pridham and Jensen of Magnavox. The first versions of their loud-speaker consisted simply of a straight piece of copper wire placed between the poles of an electromagnet. From the centre of this wire a short wooden connecting rod was glued to a diaphragm [2]. At first, sound was brought to the ears of a listener using listeningear tubes of the stethophone type which were connected to the airspace in front of the 
diaphragm. Then, quite by accident, a phonograph horn was inserted into the sound box, the results were amazing to the experimenters; clarity with volume resulted. The most successful version of the Magnavox loudspeaker was the R-3, introduced in 1921 or 1922 and shown in figure 3.1 and 3.2 [1].

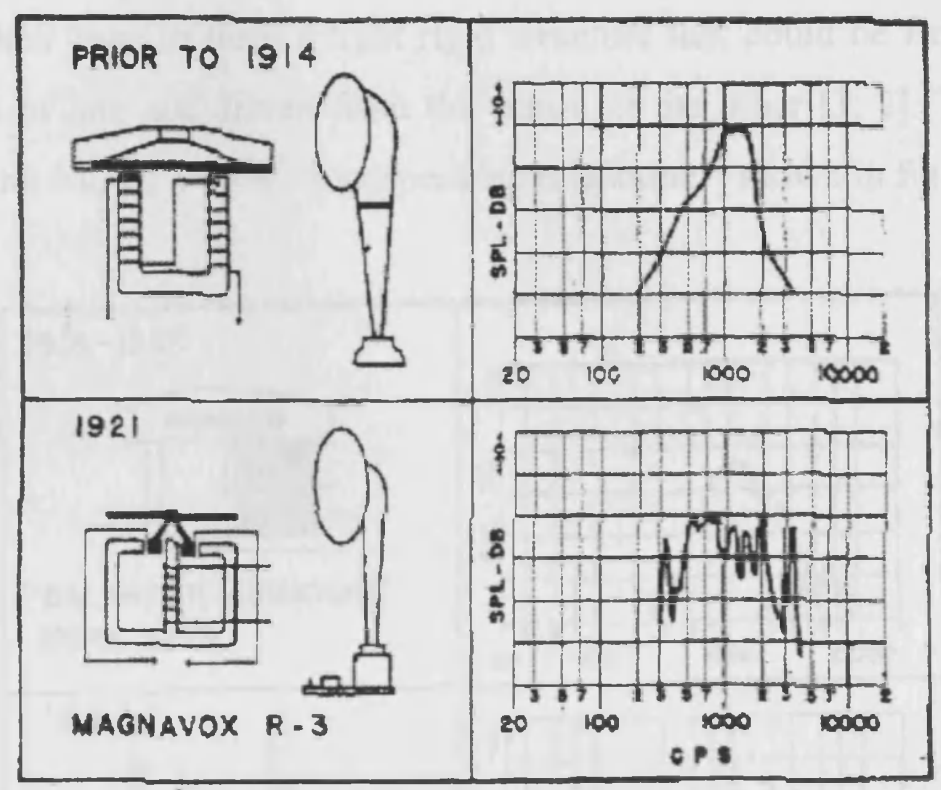

Figure 3.1: Two different loudspeaker designs during the early part of the twentieth century [1]

An improvement on the early rocking-armature transducer was the 4 air-gap balancedarmature type by Egerton (1918) that permitted greater amplitude of movement of the moving-iron type of diaphragm without distortion [1].

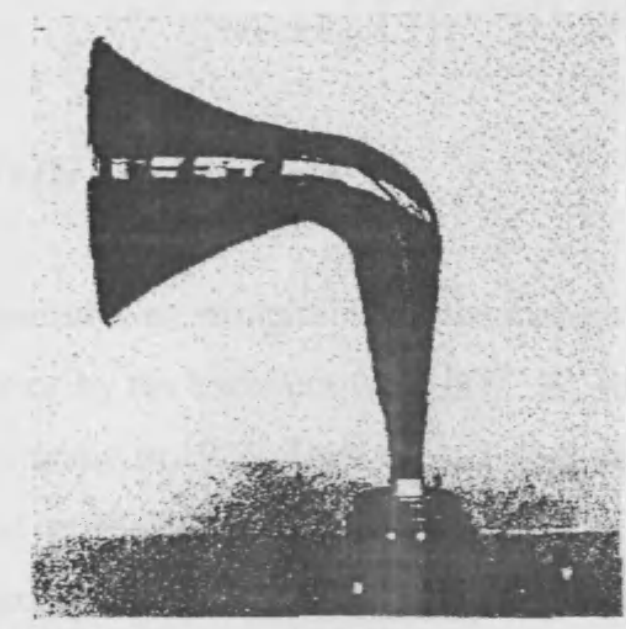

Figure 3.2: Magnavox R-3 loudspeaker (Pridham and Jensen, 1921) [1] 
After the success of the balanced armature type loudspeaker, improvements in sound reproduction machine followed by using a large radiating diaphragms instead of horns. Between 1900 and 1924, important developments were made in the choice of materials, dimensions, and methods of manufacture of sound-radiating diaphragms. It was Ricker of Western Electric who described the use of two large wide-angle cones cemented at their base to form a light rigid structure that could be freely suspended from the apex of one and driven from the centre of the other [1,2]. This led to the Western Electric Model 540-W "loudspeaking telephone," shown in figure 3.3 [1].

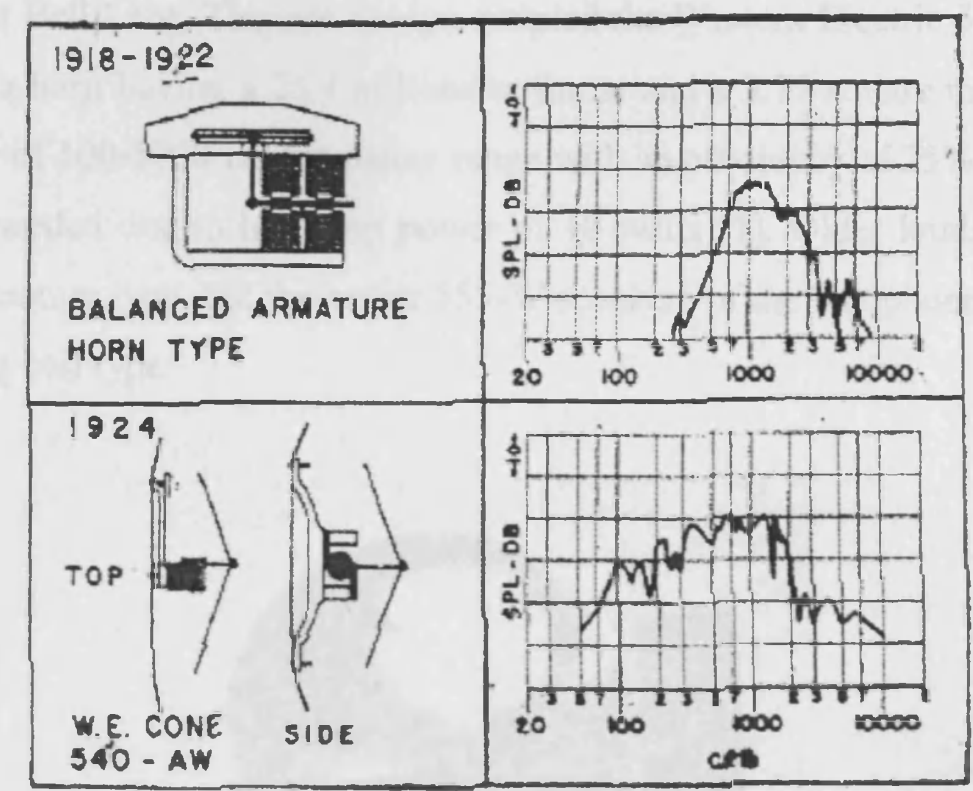

Figure 3.3: Upper: Balanced-armature transducer connected to short exponential horn. Lower: Balanced armature type unit utilizing two large paper cones joined at outer edges. [1]

\subsection{Improvement on Early Loudspeakers}

The present-day loudspeaker was brought out of the category of being a loud device into being a faithful device by the excellent work of C. W. Rice and E.W. Kellogg of the General Electric Company in 1925. They made a very careful and thorough study of direct radiation, and capitalized on the importance of locating the resonance frequency of the diaphragm at the bottom of the frequency spectrum so that the diaphragm vibrates as a mass-controlled device [1,2, and 3]. Under these conditions, a flat response was obtained in the frequency region above the resonance frequency. 
Rice and Kellogg assured themselves of a market for their loudspeaker by developing a power amplifier yielding one-watt of available audio power. The combination loudspeaker-amplifier-cabinet was sold, in 1926, under the trade name of "Radiola Loudspeaker Model 104". Other companies soon came forth with direct-radiator loudspeakers. Magnavox put one on the market in 1927, and Jensen claims the first high-efficiency auditorium loudspeaker in 1928.

In the similar period, Vitaphone sound system for motion pictures used a new speaker developed at Bell Labs. The new design coupled the Western Electric 555-W speaker driver with a horn having a 25.4 millimetre throat and a 3.72 square metre mouth. It was capable of $100-5000 \mathrm{~Hz}$ frequency range with an efficiency of $25 \%$ (compared to $1 \%$ today) needed due to low amp power of 10 watts [1]. Older loudspeakers were balanced armature type, but the newer 555-W speakers of the Vitaphone (Fig. 3.4) [1] were moving coil type.

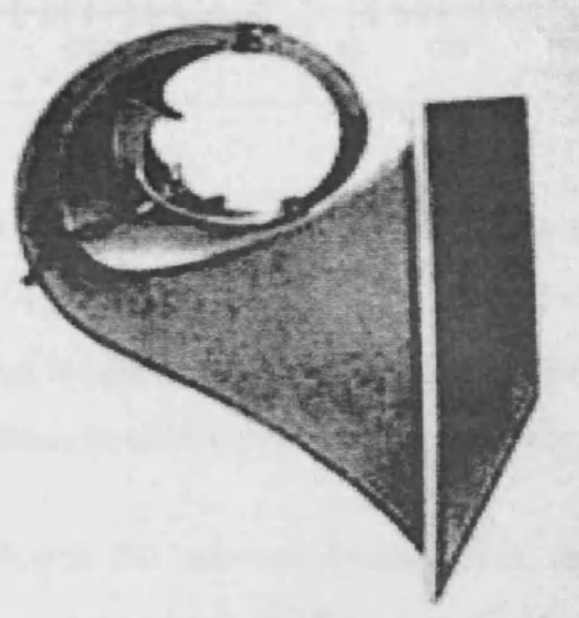

Figure 3.4: Vitaphone Speaker [1]

Coaxial speakers also came into existence by Herman J. Fanger in 1928. The new loudspeaker design composed of a small high frequency horn with its own diaphragm nested inside or in front of a large cone loudspeaker, based on the variable-area principle that made the centre cone light and stiff for high frequencies and the outer cone flexible and highly damped for lower frequencies. 
In 1931 permanent-magnetic loudspeakers were first commercially available from the Jensen Manufacturing Company. Many other developments then followed in loudspeaker design. The loudspeakers developed at this time were mostly of multicoil, multi-diaphragm type. One of these, the double voice coil by Olson in 1934 is shown in figure $3.5[1]$.

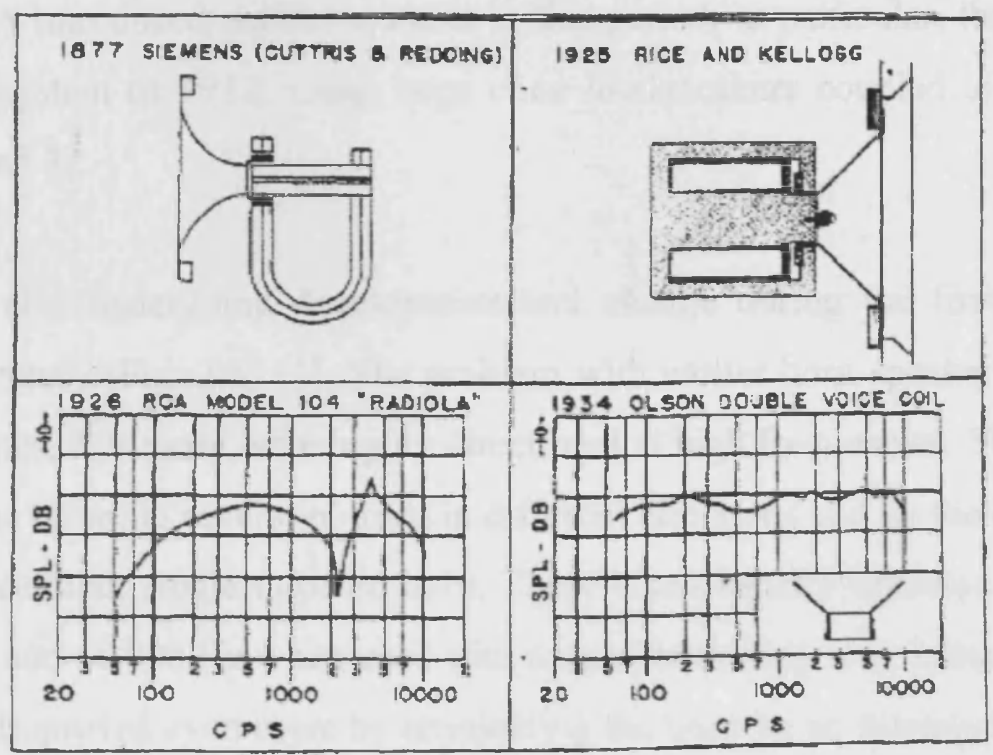

Figure 3.5: Upper left: Design of direct-radiator, moving-coil from Siemens' patent application. Upper right: Design of Rice and Kellogg of 1925 left: Response of 1926 version of Rice-Kellogg loudspeaker. Lower right: Response and construction of Olson double-voice coil loudspeaker [1]

In 1931, Bell Labs developed the two-way loudspeaker, called "divided range". The high frequencies were reproduced by a small horn with a frequency response of 3000$13,000 \mathrm{~Hz}$, and the low frequencies by a 3048 millimetre dynamic cone direct-radiator unit with a frequency response within $5 \mathrm{db}$ from $50-10,000 \mathrm{~Hz}$. By 1933 , a triple-range speaker had been developed adding Western Electric No. 555 driver units as the midrange speaker. For the low frequency range $40-300 \mathrm{~Hz}$, a large moving coil-driven cone diaphragm was employed in a large baffle expanding from a 3048 millimetre throat to a 15240 millimetre mouth over a total length of 30480 millimetre. This 3way system was introduced in motion picture theatres as "Wide Range" reproduction [1]. 
In 1933, the Jensen Company brought out the first commercially available tweeter units. That same year, the Western Electric Company also introduced the Bostwick Type 596 tweeter unit. These units extended the frequency range of reproduced sound up to better than $12,000 \mathrm{~Hz}$, and brought about the widespread use of two-way systems. The low-frequency portion of the spectrum was extended below the cut-off frequency of the coiled horn through the development of high-efficiency directradiator units such as the Jensen DA-4, thus bringing into existence the first three-way systems. RCA introduced similar systems in this period, in particular, the Radio City Music Hall System of 1932, using large cone loudspeakers coupled to exponential horns [1, 2 and 3].

Horns were also undergoing development and change during the first part of the nineteenth century (Fig. 3.6) [1]. The problem with earlier horn speakers, with large mouth openings, that those were highly directional at high frequencies. This difficulty was overcome by using several pointed in different directions and by the development of the multi-cellular, single throated horn. These horns usually operated in the range between 400 and $10,000 \mathrm{~Hz}$ when used with a suitable driving unit. Later on, the horn speaker was improved even more by terminating the horn by an acoustic lens that has more uniform radiation pattern than the multi-cellular horn. This type of horn was first described by Kock and Harveyo of Bell Telephone Laboratories in 1949 [1, 2].

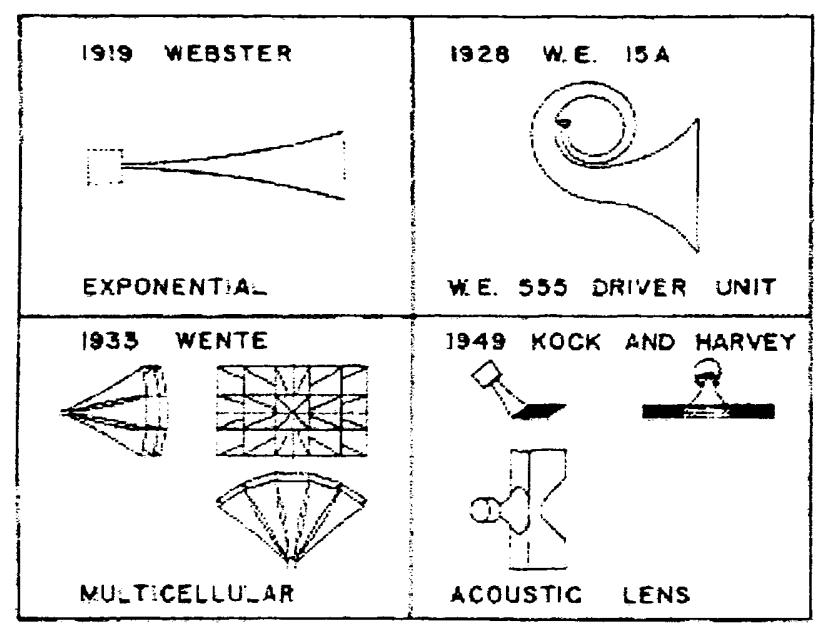

Figure 3.6: The evolution of horn type loudspeakers [1]

In the same time when horn type speakers were getting improved, development was also in progress in the field of direct-radiating loudspeakers. The developments of baffles for direct radiator loudspeakers are shown in figure 3.7 [1]. 

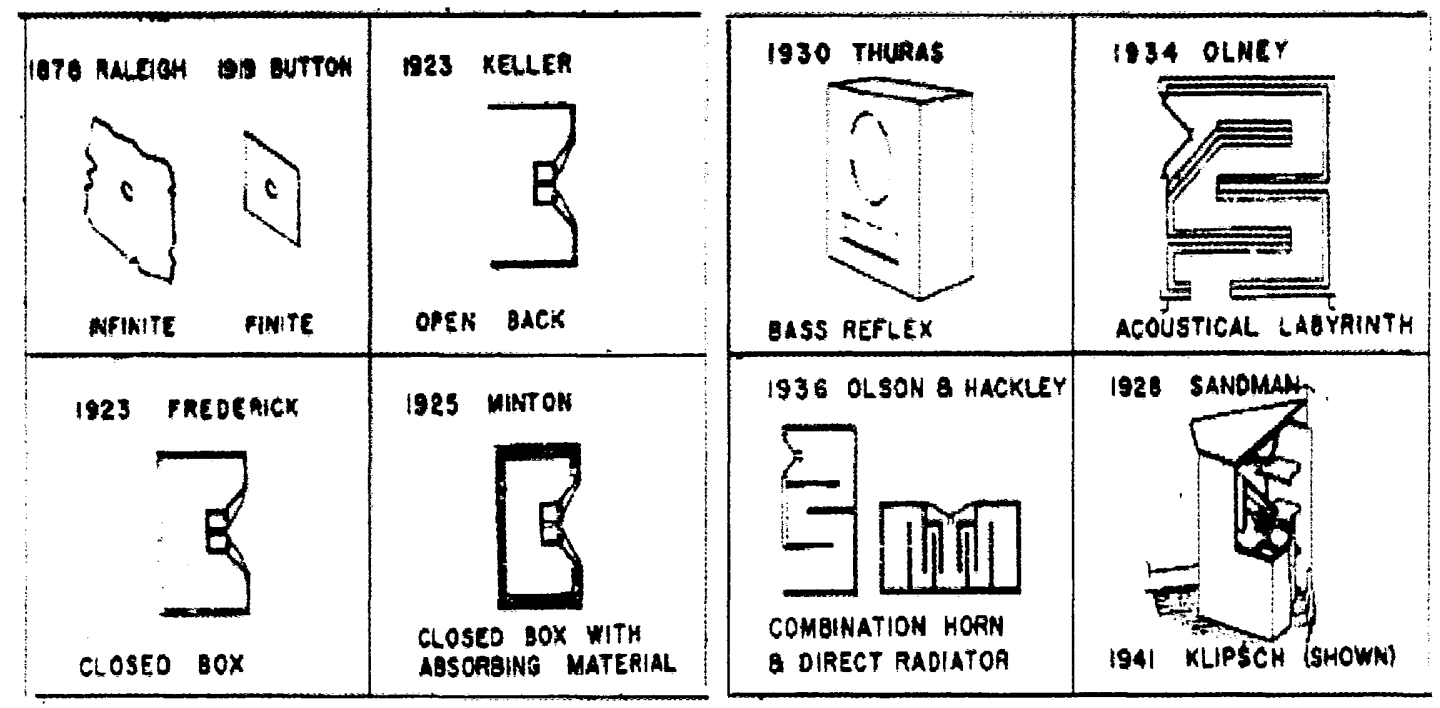

Figure 3.7: Improvement of baffles in the early nineteenth century [1]

The talking motion picture was mainly responsible for the development of efficient high-fidelity, higher-power loudspeaker systems. There were two contributors who dominated the sound motion picture scene prior to 1938, namely the Western Electric Company and the Radio Corporation of America. The first RCA systems consisted of a vertical column of Rice-Kellogg direct-radiator loudspeakers located on either side of the screen. Although these units sounded very good, their efficiency was very low. In 1926-1927 the Western Electric Company came out with the Type 555W receiver. This horn driver unit, when used with a 30480 millimetre to 45720 millimetre long exponential horn, was very efficient in large theatres in the frequency range of 100 to $5.000 \mathrm{~Hz}$. Its electrical power handling capacity was 30 watts, and its efficiency was about 50 per cent throughout the frequency range between 150 to $4,500 \mathrm{~Hz}$ [2].

A milestone in loudspeaker design was the Altec-Lansing Model 604 duplex radiator (Fig. 3.8) [3] that was introduced in the early 1940s and described by James B. Lansing in a paper published in 1943 [4]. The 604 combined a highly efficient 3810 millimetre woofer (a loudspeaker for producing low frequency sounds) with a highfrequency compression driver and horn. The high-frequency driver was planted onto the back of the woofer magnet and the horn throat travelled through the woofer's centre pole piece. When it was introduced the 604 was intended primarily as a broadcast and recording studio monitor speaker, in which capacity it became an established standard [5]. 


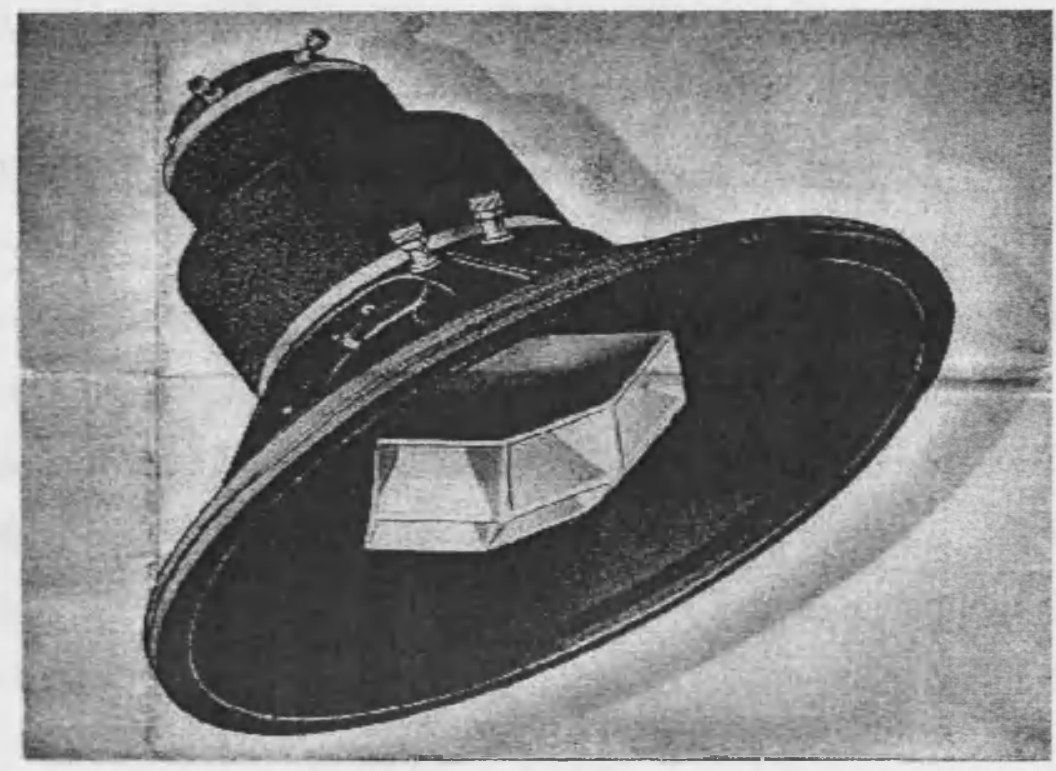

Figure 3.8: Altec-Lansing Model 604 duplex radiator Loudspeaker [3]

Another milestone in loudspeaker design was the Klipsch corner horn loudspeaker. This loudspeaker, invented in 1946, was described as "A high quality loudspeaker of small dimensions" by its inventor [6]. It was an overnight success that remains a contemporary standard. The design was a beautiful example of good theory combined with sudden insight.

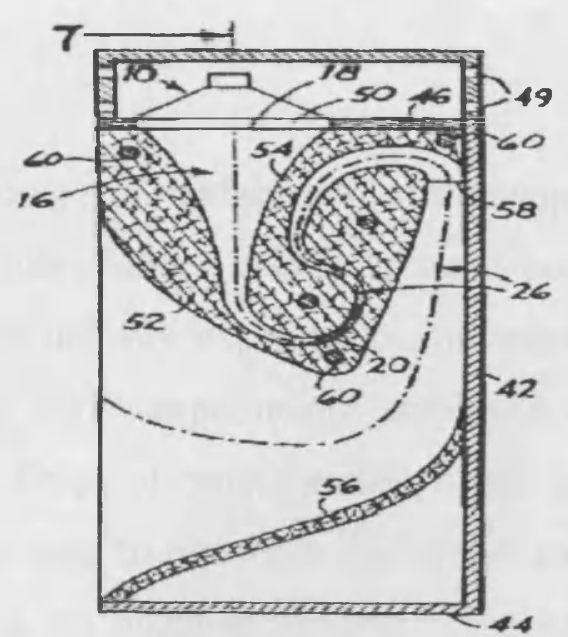

Figure 3.9: A patented folded horn featuring a gradually decreasing then increasing cross-sectional area. [4]

Paul Klipsch, the inventor, realized that the room boundaries themselves would serve as a horn mouth; all that needed to be done was to design a suitable folded throat section. In contrast to the large theatre horns of the time, the Klipsc horn was about a 
tenth the size and had powerful low frequency response that extended about an octave lower. Audiophiles were thrilled to discover that there was music below $55 \mathrm{~Hz}$.The Klipsch horn inspired countless other folded horn designs that still keep evolving. Figure 3.9 [4] illustrates one of the scores of patented variations.

An important invention in the early 1940's was the "bass reflex" loudspeaker system. The new loudspeaker employed vented box enclosure to enhance bass response. Patented variants of the vented loudspeaker enclosure pushed the limits of man's imagination to new frontiers. Because of its simple geometry the vented box became a favourite of home constructors, but results were often disappointing. This was mainly because the interaction between loudspeaker-box-vent was not well understood.

However, primarily through the use of electrical analogue circuits, the behaviour of vented systems became better understood. Locanthi's 1952 paper [7] on electrical analogies was used by at least two manufacturers to design better vented systems. In 1951, specific loudspeaker driver parameters and appropriate enclosure "alignments" were described by Neville Thiele [8] and later refined by Richard H. Small. Today, Thiele-Small parameters are routinely published by loudspeaker manufacturers and used by consumers to design systems with predictable low-frequency characteristics.

\subsection{Surround Sound}

For decades, music recording and reproduction was monophonic. However, there was a real desire for a multiple-channel system to more accurately represent the live musical experience. Initial industry experiments with stereo recording took place in the early 1930s. These early experiments employed an "infinite" number of microphones deployed in a type of curtain in front of the recording musicians, and an equal number of speakers used to reproduce the sound. Later, loudspeaker designers discovered that they could use phantom imaging to reproduce the stereo soundstage with just two speakers.

The idea of stereo sound came into existence in the 1930's. Researchers in loudspeaker industries developed this idea after noting that people listened to sound through one speaker but had two ears. Therefore a new loudspeaker system called 
'stereophonic' was introduced because of its ability to give a spatial sense corresponding to stereophonic vision.

Stereophonic sound, commonly called stereo, is the reproduction of sound, using two independent audio channels, through a pair of widely separated speaker systems, in such a way as to create a pleasant and natural impression of sound heard from various directions as in natural hearing. It is often opposed to mono, where audio is in the form of one channel, often centered in the sound field. The stereo sound arrangement inspired better music experience for many people especially in movie theatres.

Stereo sound reproduction has its origins in the U.K. where Blumlein developed a system in 1931 that could place a sound in the horizontal (azimuth) plane by using an appropriate combination of delay and level differences [9]. His work focused on the development of microphone techniques that would allow the recording of the amplitude and phase differences necessary for stereo reproduction. A few years later, Fletcher, Steinberg, and Snow, at Bell Laboratories in the U.S., discussed a 'wall of sound," and concluded that an infinite number of microphones would be necessary to capture a sound scene [10,11 and 12]. At the reproduction end, an infinite number of loudspeakers would be required to reconstruct the sound field in a manner similar to the 'Huygens principle of secondary wavelets' [13]. The Bell Labs researchers proposed a practical implementation based on a three-channel system consisting of left, centre, and right channels in the azimuth plane. Such a system could represent the lateralization and depth of the desired sound field with acceptable accuracy. The first such stereophonic three-channel system was demonstrated in 1934, with the Philadelphia Orchestra performing remotely for an audience in Washington, D.C., over wideband telephone lines.

From 1940 to 1970 , the progress of stereophonic sound was paced by the technical difficulties of recording and reproducing two (or more) channels in synchronization, and by the economic and marketing issues of introducing new audio media and equipment. To a rough approximation, a stereo system cost twice as much as a monophonic system, since a stereo system had to be assembled by buying two preamplifiers, two amplifiers, and two speaker system. Additionally, the surround 
sound experience was missing from the stereo sound system becasuse all the sound was radiating from in front and between the spakers.

Taking a more realistic and affordable approach to surround sound reproduction, quad, as it was called, was born, which used four speakers: left and right speakers in front of the listener as in conventional stereo and left and right speakers behind the listener to create the sensation of being "surrounded by sound".

The desire to reproduce a 360-degree sound field led to the development of fourchannel or quadraphonic recording. Quadraphonic sound officially launched in 1969 with the release of the first consumer-level four-channel reel-to-reel tape deck. Soon the quadraphonic process was being applied to both eight-track tapes and vinyl records.

By the early 1970s multiple quadraphonic technologies were competing in the marketplace. JVC's CD-4 system, introduced in 1971 for vinyl records, employed four discrete channels of audio information - front left, front right, rear left, and rear right. The $S Q$ and $Q S$ systems, introduced in 1972 by CBS and Sansui, respectively, were both matrix technologies for vinyl records, in which the rear channel information was matrixed into the two front channels and then separated out by a surround decoder. RCA's Quad-8 format, introduced in 1970, was a discrete format designed specifically for eight-track tape players [14].

The confusion generated by these competing technologies, along with the high cost of four-channel amplifiers and additional rear speakers, led to the abandonment of quadraphonic sound by the end of the decade.

Around 1977 the movie industry was searching to see if there is any way to deliver something a little more exciting than the simple stereo then standard in movie theatres. Dolby, who had licensed some of the original quad patents, turned the speaker configuration into a diamond shape rather than a rectangle. Left, Right, Surround Left, and Surround Right became Left, Centre, Right, and Surround.

By providing a special encoder, it was possible to merge, or encode, four source channels into the more easily delivered two-channel recording formats, and then play 
them back in the theatre with a decoder that derived four channels again. Thus was born Dolby Stereo. The configuration made it possible to isolate dialog to the centre speaker, but the surround channel was monaural instead of stereo, unlike the original quad.

During the 1970's and 1980's, Dolby surround sound technology dominated the consumer market. However, despite the huge success of Dolby stereo, it was suffering from limitations like that of its predecessor - stereo and quad. The main limitation was the positioning of the speaker and its effect on 'sweet spot'. A small change in the positioning of the speaker or the listener could greatly distort the surround sound experience.

Later, in the 1990s, when digital penetrated the consumer market in DVD players and digital receivers, Dolby released Dolby Digital, offering high-quality 5.1 channel performance. Both systems provided, for the first time, five discrete high-quality audio channels (Left, Centre, Right, Left Surround, and Right Surround) plus the .1 channel. This bandwidth-limited channel was dubbed LFE for low-frequency effects (like explosions, bombs, and jet takeoffs) and in a typical home theatre system is connected to a subwoofer. Each of the five speakers had to be positioned properly, wired to the main amplifier or receiver, and balanced. However, the problems associated with correct speaker positioning, listening environment and sweet spot location remained unchanged and therefore, for many people this was the beginning and the end of their surround sound experience.

In the meantime, some researchers, mainly those involved with high-end audio, were wondering why stereo, and even surround sound, fell short of sounding like the real thing. What came out from their research is that people perceive sound as arising in three-dimensional space, not just in a flat horizontal plane between speakers. People can distinguish if a sound is above, below, in front, to the side, or behind them. In fact, many people can resolve the location of a sound within one degree of horizontal azimuth. 
It turns out that what the early designers of stereo and quad systems had failed to understand is that locating a sound in space involves more than amplitude, time, and phase differences (the traditional factors that account for stereo localization).

The ear-brain system can determine the direction of a sound source, because of its uncanny ability to utilize changes in the frequency distribution of a complex sound as it arrives at the ear canal. This distribution changes as the sound source changes position relative to the ear, primarily as a result of the outer ear which acts as a baffle and resonator specifically to create the effect [14].

The brain interprets these subtle variations in frequency distribution as directional clues, rather than as changes in the timbre of the frequency of the sound source. This permits the ear/brain system to resolve the direction of a sound source in threedimensional space. Without this capability there could be no surround sound. People would be unable to distinguish between sounds coming from the front and the rear speakers.

These direction-based changes in frequency distribution caused by the pinna, plus others caused by head shadowing and reflections, are now collectively referred to as head related transfer functions (HRTFs) (Fig. 3.10) [14].

The realism of stereo reproduction was much enhanced by using these curves and filtering the difference portion of a stereo signal, which contains directional and ambient information representing the acoustic space of the original recording. This proprietary process, called SRS (Sound Retrieval System), was the first of the HRTF based 3-D sound enhancement systems. When SRS is activated, the listener perceives a sound field that extends well beyond the horizontal position of the speakers and curves around the head to an angle of about 180 degrees [14]. With multichannel virtualizers, only two speakers do the job of multispeaker surround systems. 


\section{Tricking the Brain}

Virtual surround systems use a frequency response curve like this one,

synthesized from measured front and side head-related transfer

functions (HRTFS). The curve is used to modify the energy distribution

so that sound from the speakers in front is perceived as coming also

from the listener's side.

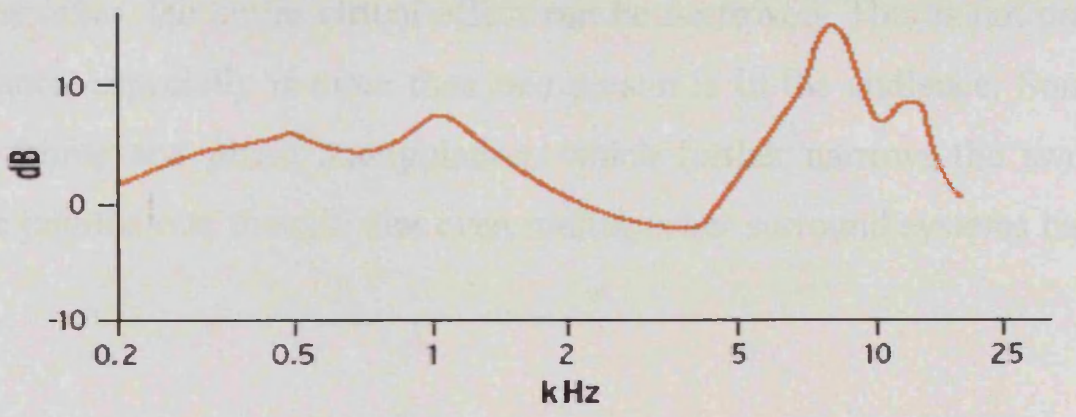

Figure 3.10: Frequency Response Curve from HRTFs [14]

Both Dolby digital and DTS quickly adopted the HRTF techniques similar to SRS to map the 5.1 discrete channels of sound (Left, Centre, Right, Left Surround, Right Surround, and low-frequency effects) into virtual space, creating the perception of sound sources to the sides and behind a listener with no physical speakers there. Different techniques were used by other systems as well, but the goal of all the systems was the same- to reproduce a full surround sound field from the two-speaker systems that people commonly had in their homes.

Using the virtual loudspeaker technology, some innovative loudspeakers have been invented. The system designed by the Cooper-Bauck Corp. and now marketed by Harman International Industries Inc. as VMAx, can persuade listeners in the sweet spot that they have entered another acoustic space entirely. Other systems, such as the 3-D positioning of QSurround from Qsound Labs Inc. can literally make the listener feel someone is creeping up from behind, though this effect is limited to a very small sweet spot.

However, as with anything, there are limitations. There are basically two issues: sound quality and the sweet spot. In addition to HRTF processing, virtualization systems use a technique called interaural crosstalk cancellation (ICC). ICC attempts to cancel the crosstalk from the right speaker to the left ear and vice versa, by isolating 
the sounds from the left speaker to the left ear and the right to the right ear. While this can be effective, it normally results in a very narrow sweet spot (this is the listening location relative to the speakers where the virtualization effect can be properly heard).

In many systems, if listeners simply turn their heads, or move as little as $3 \mathrm{~cm}$ to one side or the other, the entire virtual effect can be destroyed. This is not practical in a home theatre, especially if more than one person is in the audience. Some systems also use timing and phase manipulation, which further narrows the sweet spot. It should be pointed out, though, that even multispeaker surround systems have a sweet spot.

The evolution of home audio, from stereo loudspeaker to virtual surround sound speaker, is shown in figure 3.11 [14]. 


\section{Evolution of Home Audio}

Stereo in the late 1920 s and early 1930 s relied on two speakers [top]. Ouad, in the 1970s, was the first atternpt to surround a listener with sound. Today's 5.1 home-surround systems are like those used in movie theaters, delivering five audio channels through traditional speakers plus a bandwidth-limited channel through a subwoofer. Virtual surround systems [bottom] revert to two speakers but add signal processing to create phantom speakers.

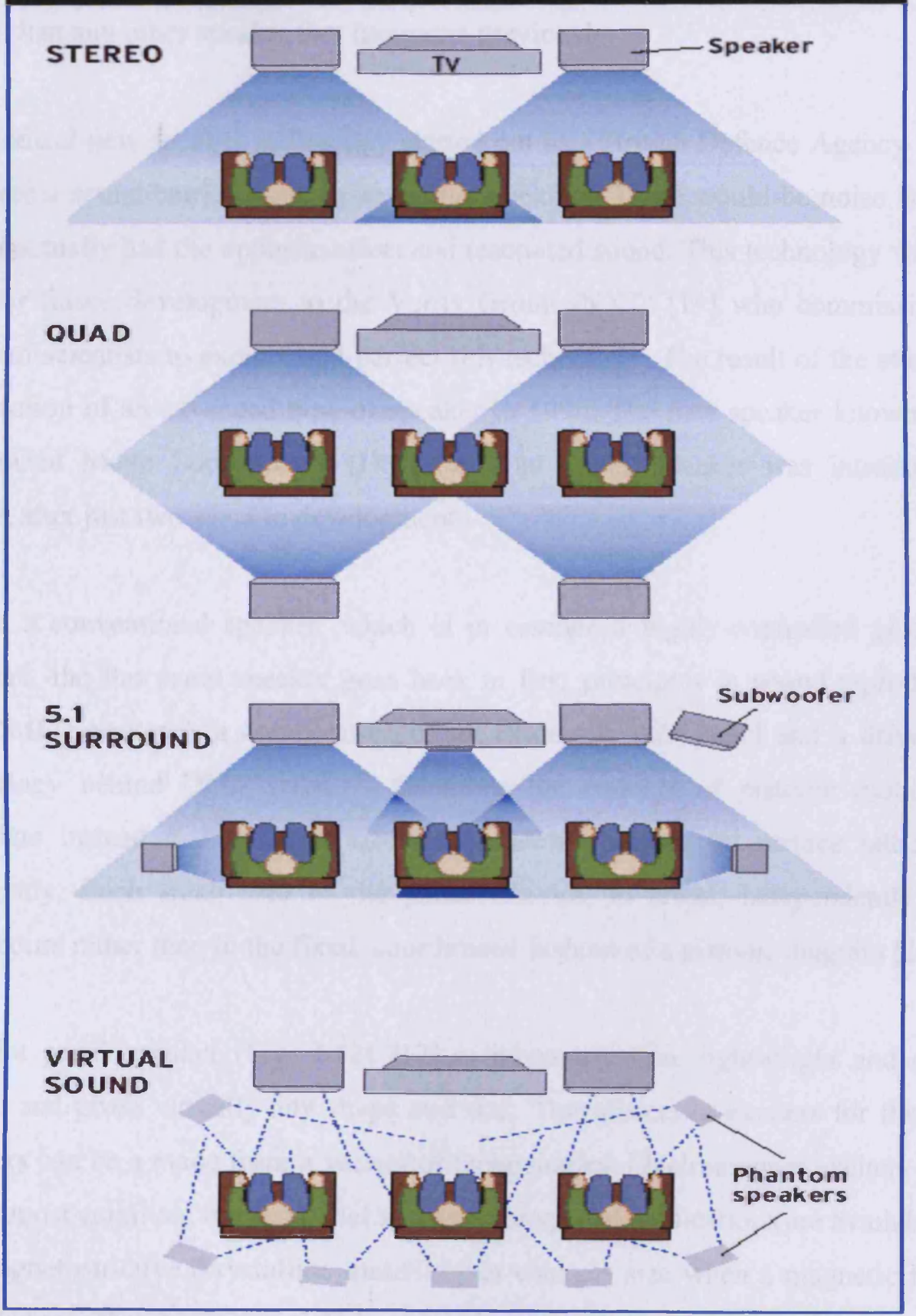

Figure 3.11: Evolution of Home Audio [14] 


\subsection{Flat Panel Speaker}

The newest and possibly the most revolutionary advance in speaker technology since the advent of the moving coil speaker has been designed, perfected and licensed for world-wide manufacturing by a company named NXT plc (New Transducers Ltd) [15]. This flat panel speaker design relies on an entirely different theory to create sound than any other speaker that has come previously.

This radical new speaker technology started out as a British Defence Agency project to create a sound barrier panel in jet fighter cockpits. These would-be noise isolation panels actually had the opposite effect and resonated sound. This technology was later sold for future development to the Verity Group (NXT) [15] who commissioned a group of scientists to explore and perfect this technology. The result of the study was the creation of an advanced type of speaker in 1996. The new speaker known as the Distributed Mode Loudspeaker (DML) or Flat Panel Speaker was introduced to market after just two years in development.

Unlike a conventional speaker, which is in essence a highly-controlled piston-like structure, the flat panel speaker goes back to first principles in sound reproduction. The DML Speaker is a combination of an extremely stiff panel and a driver. The technology behind DML speakers abandons the concept of pistonic motion and considers instead a diaphragm vibrating randomly across its surface rather than coherently. Each small area of the panel vibrates, in effect, independently of its neighbours rather than in the fixed, coordinated fashion of a pistonic diagram [16].

The flat panel speaker (Fig. 3.12) [17] is inherently thin, lightweight and can be curved and given virtually any shape and size. Transducers or exciters for flat panel speakers can be a made from a variety of technologies. Electromagnet exciters are by far the most common, but flat panel speakers for special applications are available that use magnetostrictive (crystalline material that changes size when a magnetic field is passed across them) exciters and piezoelectric (crystalline material that changes shape when a electric field is passed across them) exciters. 
Unlike the conventional loudspeaker, flat panel speaker generates sound in all directions. A non-directional device allows both speakers positioning and listener positions to be much less critical. In addition, because the flat Panel Speaker behaves in effect like thousands of separate point sources, the sound radiation surface is quite large and hence it creates an even sound field in a given space. More details on the flat panel construction, advantages and limitations are discussed in chapter 4 .

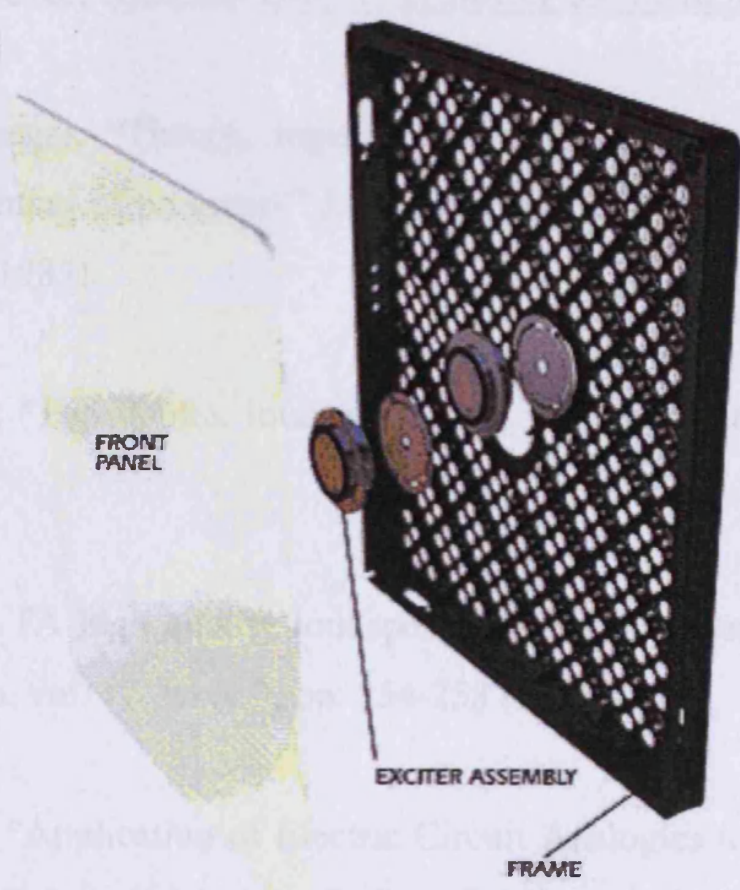

Figure 3.12: Inside the flat panel loudspeaker [17] 


\section{References:}

[1] Loudspeaker History - http://history.acusd.edu/gen/recording/loudspeaker.html

[2] L. Beranek, "Loudspeakers and Microphones," IRE Transactions on Audio, vol. 3, Issue 1, pp. 13-20 (1955).

[3] Duplex Loudspeaker Systems- http://alteclansingunofficial.nlenet.net/Duplex.html

[4] G. L. Augspurger, "Theory, ingenuity, and wishful wizardry in loudspeaker design-A half-century of progress?" J. Acoustical Society of America, vol. 77, Issue 4, pp. 1303-1308 (1985).

[5] J. B. Lansing, "The duplex loudspeaker," J. Communications, vol. 23, pp. 22 (1943).

[6] P. W. Klipsch, "A high quality loudspeaker of small dimensions," J. Acoustical Society of America, vol. 17, Issue 3, pp. 254-258 (1946).

[7] B.N. Locanthi, "Application of Electric Circuit Analogies to Loudspeaker Design Problems," IRE Transactions on Audio, PGA-6 (1952), reprinted J. Audio Engineering. Society, vol. 19, Issue 9, pp. 775-785 (1971).

[8] A. N. Thiele, "Loudspeakers in Vented Boxes," Proc. IRE Australia, vol. 22, pp. 487-508 (1961); reprinted J. Audio Engineering Society, vol. 19, Issue 5, pp. 382-392 (1971).

[9] A.D. Blumlein, "Improvements in and relating to sound-transmission, soundrecording and sound-reproducing systems," U.K. Patent no.394325 (1931).

[10] H. Fletcher, "Auditory perspective-Basic requirements," J. Electrical Engineering., vol. 53, pp. 9-11 (1934). 
[11] J.C. Steinberg and W. B. Snow, "Physical Factors," J. Bell Systems Technology, vol. 13, pp. 245-258 (1934).

[12] W.B. Snow, "Basic principles of stereophonic sound," IRE Transactions on Audio, vol. 3, Issue 2, pp. $42-53$ (1955).

[13] S. Y. Nikitin, S. A. Ahmanov, "Physical Optics," Oxford University Press, 1997

[14] A. Kraemer, "Two speakers are better than 5.1," IEEE Spectrum, vol. 38, Issue 5, pp. 70-74 (2001)

[15] NXT plc (New Transducers Ltd), Cygnet House, Kingfisher Way, Huntingdon, PE29 6FW, UK

[16] H. Azima, "NXT Technology," NXT technical paper, 1996.

[17] Image of Flat Panel Speaker-

http://media.popularmechanics.com/images/tb 9809EFSTE.jpg 


\subsection{Introduction}

Conventional designs and flat panels are the two major areas of interest in the loudspeaker industry. Until recently, conventional loudspeakers were the dominant technology in loudspeaker market. The highly efficient pistonic motion of the conventional diaphragm, especially in the low frequency region, made it a huge success since its invention in the 1920 's. Countless variations of the early design, innovative enclosures, cross-over network, improved amplifiers and surround sound technology have all enriched the conventional loudspeaker technology over the years. In this chapter, the electrical and physical parameters of the conventional loudspeakers are discussed and the advantages and the limitations of using such loudspeakers are also explained in this chapter.

The introduction of flat panel technology in the late 1990's was a major invention as far as the loudspeaker industry is concerned. The conventional sound reproduction mechanism has been replaced by a completely new idea that, although new, is based on the basic concept of sound propagation. It was an alternative way of reproducing sound to the traditional 'pistonic' loudspeaker. Instead of a coherent movement of the loudspeaker diaphragm in a conventional loudspeaker, the random vibration of a panel in a flat panel speaker could create high quality non-directional sound especially at high frequency.

In a conventional loudspeaker, the diaphragm - whatever its shape and whatever its means of motivation - is designed to act as a piston, at least over much of its operating range. It is intended to move as a rigid, coordinated whole to displace the air at its surface. Flat panels operate in an entirely different way, by initiating and exploiting multiple, organised bending resonances in the panel. This distributed-mode behaviour results in complex vibration, which in turn creates the sound.

The distributed mode loudspeaker was trendy, slim and hence required less space. At the same time, the efficiency of high frequency sound reproduction made flat panel 
speakers a serious contender to conventional loudspeakers. However, the flat panel loudspeaker, itself, suffers from various limitations. This chapter discusses the advantages of using flat panel loudspeakers over conventional loudspeakers and also the limitations and disadvantages of distributed mode loudspeakers.

It should be noted that for the comparative analysis and description of conventional and flat panel loudspeakers, only the electromagnetic transduction mechanism has been considered. Since electromagnetic transducers have been the most efficient in terms of power transfer and also the most popular transduction mechanism for loudspeakers, the electromagnetic approach in loudspeaker design have been analyzed for both forms of the speaker. In addition, the advantages and limitations of the two types of loudspeakers are independent of the form of transducers used in them. Therefore the comparative analysis remains valid for all the different transduction mechanisms.

\subsection{Conventional Voice Coil Loudspeaker}

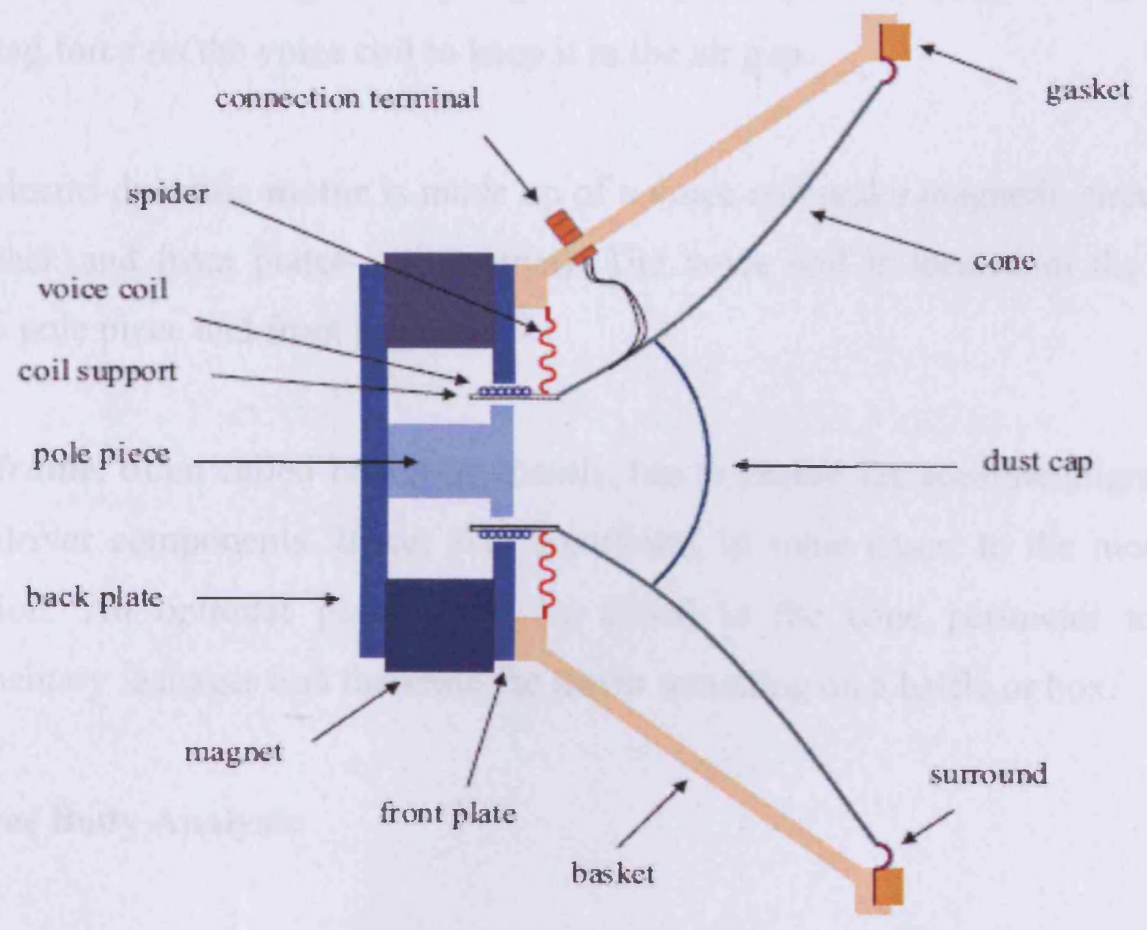

Figure 4.1: Drawing of a conventional voice coil loudspeaker [1] 
A typical voice-coil loudspeaker is illustrated in Figure 4.1 [1]. A time varying current from an amplifier drives a voice-coil motor attached to a rigid cone. The cone motion is constrained to move predominantly in the axial direction by the spider and surround (together forming the mechanical suspension), which are rigidly attached to the loudspeaker's basket. This attachment method also introduces mechanical stiffness and damping which forces the cone to rest in a nominal position when no current is fed into the voice-coil.

As shown in the figure 4.1 [1], an electro-dynamic driver comprises four parts $[1,2]$ according to their functions:

1. Made up of a cone and a dust cap, the diaphragm is the radiating component. The dust cap, in the shape of a dome or inverted dome, is used to avoid any dust or foreign particles getting into the motor.

2. The suspensions are the spider (inner part) and the surround or hinge (outer part). They play a key role in the smooth functioning of the whole system. Their main purpose consists in holding the diaphragm while guiding it axially, as well as exerting a restoring force on the voice coil to keep it in the air gap.

3. The electro-dynamic motor is made up of a voice coil and a magnetic circuit (pole piece, back and front plates and magnet). The voice coil is located in the air gap between pole piece and front plate.

4. The frame, often called basket or chassis, has to ensure the accurate alignment of all the driver components. It can also contribute, in some cases, to the motor heat dissipation. An optional gasket may be added to the cone perimeter to avoid supplementary leakages and facilitate the driver mounting on a baffle or box.

\subsubsection{Free Body Analysis}

A one-dimensional free body diagram of the loudspeaker is sketched in Figure 4.2 [3]. The cone is viewed as a rigid piston that is free to move axially. 
A z-coordinate force balance results in the governing differential equation [3]

$$
F-F_{k}-F_{R m}+F_{b}-F_{H}=m \ddot{z}
$$

where $m$ is the loudspeaker's moving mass, $\ddot{z}$ is the cone's acceleration $\left(\frac{d^{2} z}{d t^{2}}\right)$, and the forces are due to

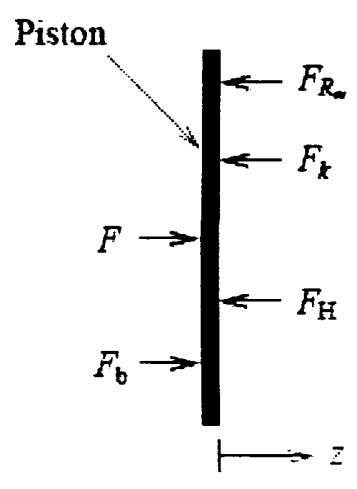

Figure 4.2: Loudspeaker Free Body [3]

$F$ : voice-coil motor,

$F_{k}, F_{R m}$ :mechanical stiffness and damping introduced by the mechanical suspension, respectively, and

$F_{H} F_{b}$ : acoustic environments the cone faces are exposed to. 


\subsubsection{Voice-Coil Motor}

Figure 4.3 [3] shows the electrical circuit diagram of the voice-coil motor where $u$ is the voltage applied across the voice-coil, and $i, L_{E}$ and $R_{e}$ are the voice-coil current, inductance, and resistance, respectively [4]. The voice-coil motion generates the backemf term $(B l(z) \dot{z})$, where $z, \dot{z}$ and $B l(z)$ are the motor's position, velocity $\left(\frac{d z}{d t}\right)$, and force factor, respectively. The force factor is the product of the magnetic flux density $(B)$ and the length of the voice-coil conductor immersed in the magnetic field $(l)$.

Assuming that $L_{e}$ and $R_{e}$ are constant while applying Kirchhoff's voltage law to the circuit yields the governing differential equation [3, 4 and 5]

$$
L_{e} \frac{d i}{d t}=u-R_{e} i-B l(z) z
$$

The force generated by the motor is proportional to the voice-coil current and is described as

$$
F(i, z)=B l(z)=F
$$

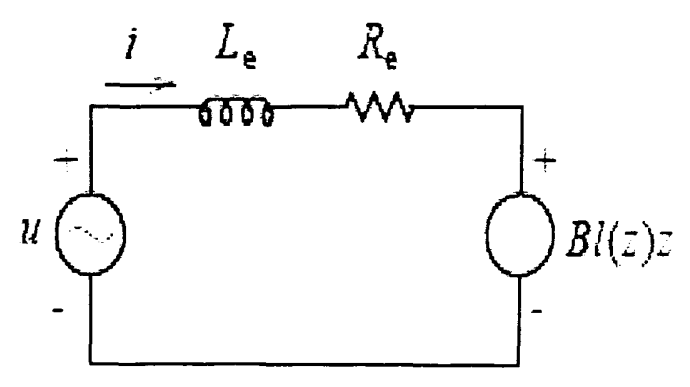

Figure 4.3: Voice Coil Motor's Electric Circuit [3]

Typically, the motor's magnetic structure produces a non-uniform magnetic field that the voice-coil moves in, such as figure 4.4 [3] illustrates. 


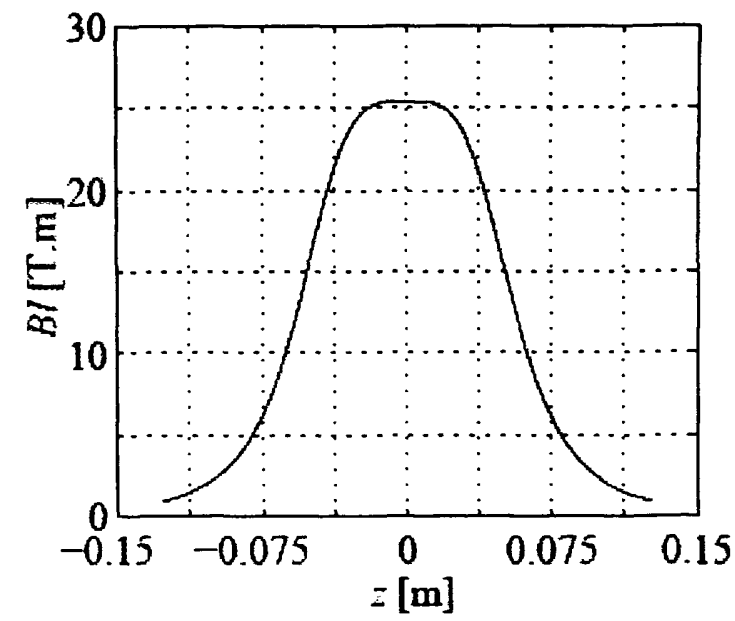

Figure 4.4: Voice Coil Motor's Force Factor [3]

Assuming a symmetric magnetic field, the nonlinearity can be parameterized by [3]

$$
B l(z)=\frac{B l_{o}}{1+B l_{1} z^{k}}
$$

where $B l_{o}$ and $B l_{1}$ are linear and non-linear force factors, respectively, and $\mathrm{k}$ is a positive, even integer $\left(B l_{o}=25.4\right.$ T.m, $B l_{1}=10^{5} \mathrm{~m}^{-4}$ and $k=4$ for figure $\left.4.4[3]\right)$.

\subsubsection{Mechanical Suspension}

The mechanical suspension comprised of the spider and surround can be modelled as a nonlinear spring and a linear damper connected in parallel. Figure 4.5 [3] shows a plot of force versus displacement for a typical stiffening spring.

This nonlinear map can be well described by the polynomial [3]:

$$
F_{k}(z)=k_{o} z+k_{1} z^{2}+k_{2} z^{3}=F_{k}
$$


where $k_{o}$ is the linear spring constant, $k_{1}$ and $k_{2}$ are the quadratic and cubic coefficients, respectively. Note that for a symmetric curve, $k_{1}=0$ is assumed $\left(k_{o}=\right.$ $3922 \mathrm{~N} / \mathrm{m}, k_{1}=0$, and $k_{2}=107 \mathrm{~N} / \mathrm{m} 3$ for Figure 4.5 [3]).

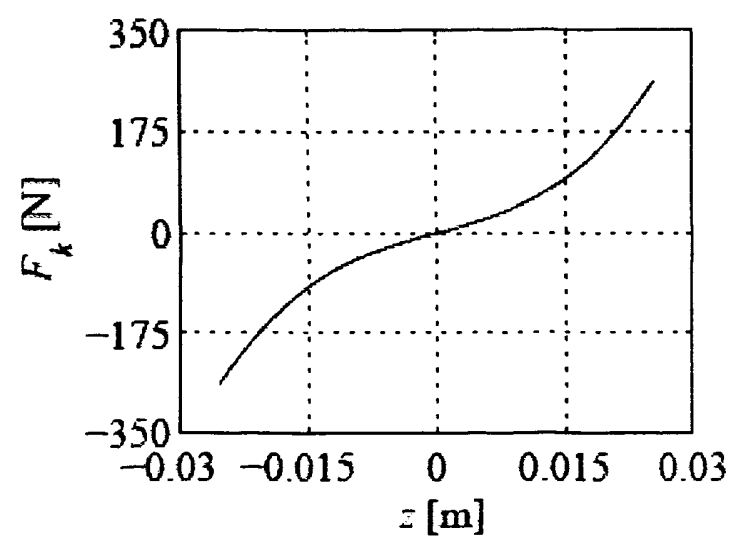

Figure 4.5: Stiffening Spring Characteristics [3]

Finally, the mechanical damping is modelled as [3]

$$
F_{R m}(z)=R_{m} z=F_{R m}
$$

where $R_{m}$ is the mechanical damping factor.

Substituting (4.3), (4.4), (4.5), and (4.6) into (4.1) and combining with (4.2) yields the nonlinear voice-coil loudspeaker equations [3]

$$
\begin{gathered}
\frac{d^{2} z}{d t^{2}}=\frac{1}{m}\left[B l(z) i-F_{k}(z)-R_{m} \frac{d z}{d t}+F_{b}-F_{H}\right], \\
\frac{d i}{d t}=\frac{1}{L_{e}}\left[u-R_{e} i-B l(z) \cdot \frac{d z}{d t}\right]
\end{gathered}
$$




\subsubsection{Enclosures}

The design of enclosures for a typical voice coil loudspeaker has been a matter of great importance since the beginning of loudspeaker industry. In a conventional loudspeaker, the front surface of the cone pushes forward to create a sound wave by increasing air pressure while the back surface of the cone lowers the air pressure. Since the wavelengths of low frequency sound are large compared to the size of the speaker, and since those low frequencies readily diffract around the speaker cone, the sound wave from the back of the cone tends to cancel that from the front of the cone (Fig. 4.6).

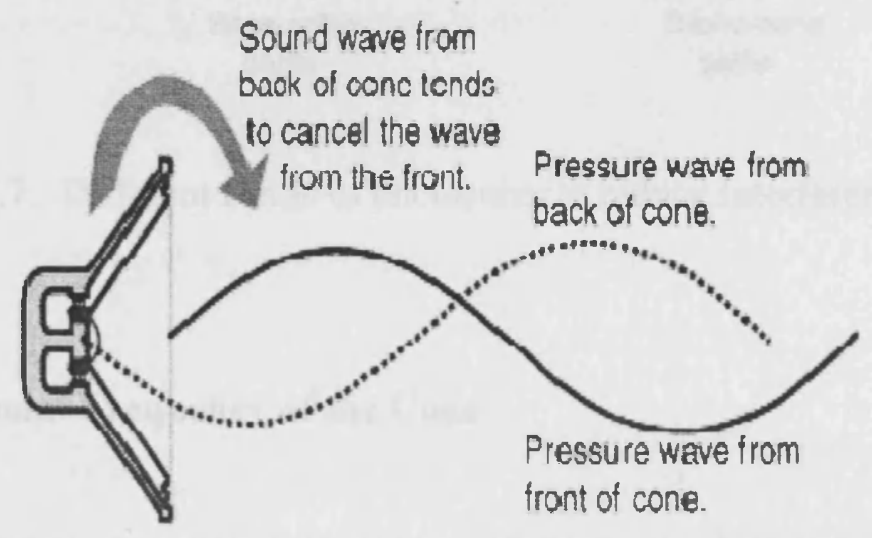

Figure 4.6: The back to front cancellation in loudspeakers [6]

For most bass frequencies, the wavelength is so much longer than the speaker diameter that the phase difference approaches $180^{\circ}$, so there is severe loss of bass from this back-to-front cancellation. This is the reason why cone-type loudspeakers employ different forms of enclosures (Fig. 4.7) to absorb the waves at back in order to produce good sound. 


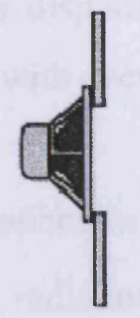

Flat baffle

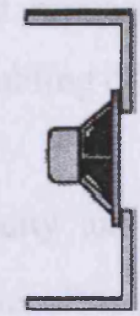

Open back baffle

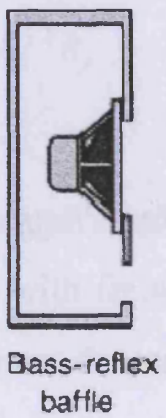

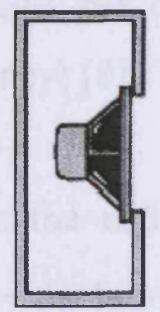

Closed

baffle

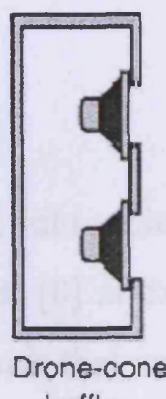

bafile

Figure 4.7: Different forms of enclosures to reduce interference of waves [6]

\subsubsection{Resonant Frequency of the Cone}

There is an inherent resonant frequency of every speaker cone assembly. This free cone resonant frequency distorts the sound by responding more strongly to signals near its natural vibration frequency. This non-uniform response changes the frequency content in terms of the relative intensities of the harmonics and thus changes the timbre of the sound. Since the cone is undamped, it tends to produce "ringing" or "hangover" with frequencies near resonance. If the resonance is in the bass range, the bass becomes "boomy". The solution is to select a cone material that has resonant frequency outside the audible frequency range.

\subsubsection{Multiple Drivers}

In acoustic terms, conventional loudspeakers are 'mass controlled' over most of their passband. The motor provides a force that is constant with frequency, the diaphragm resists with a mass (its own moving mass plus that of the air load), and so by Newton's second law of motion the diaphragm undergoes constant acceleration with frequency and the diaphragm velocity becomes inversely proportional to frequency 
[7]. As a corollary, its displacement reduces at $12 \mathrm{~dB}$ per octave with increasing frequency (i.e. quarters with every doubling of frequency) [8].

Defining $U$ as the diaphragm velocity and $R_{r}$ as the real part of the radiation impedance, the acoustic radiation power $P$ is given by the following equation [7]:

$$
P=\frac{1}{2} U^{2} R_{r}
$$

The real part of the diaphragm's radiation resistance, into which the driver dissipates acoustic power, increases with frequency (Figure 4.8) [8] at exactly the same rate as the diaphragm's displacement decreases, with the result that acoustic power output is constant [8].

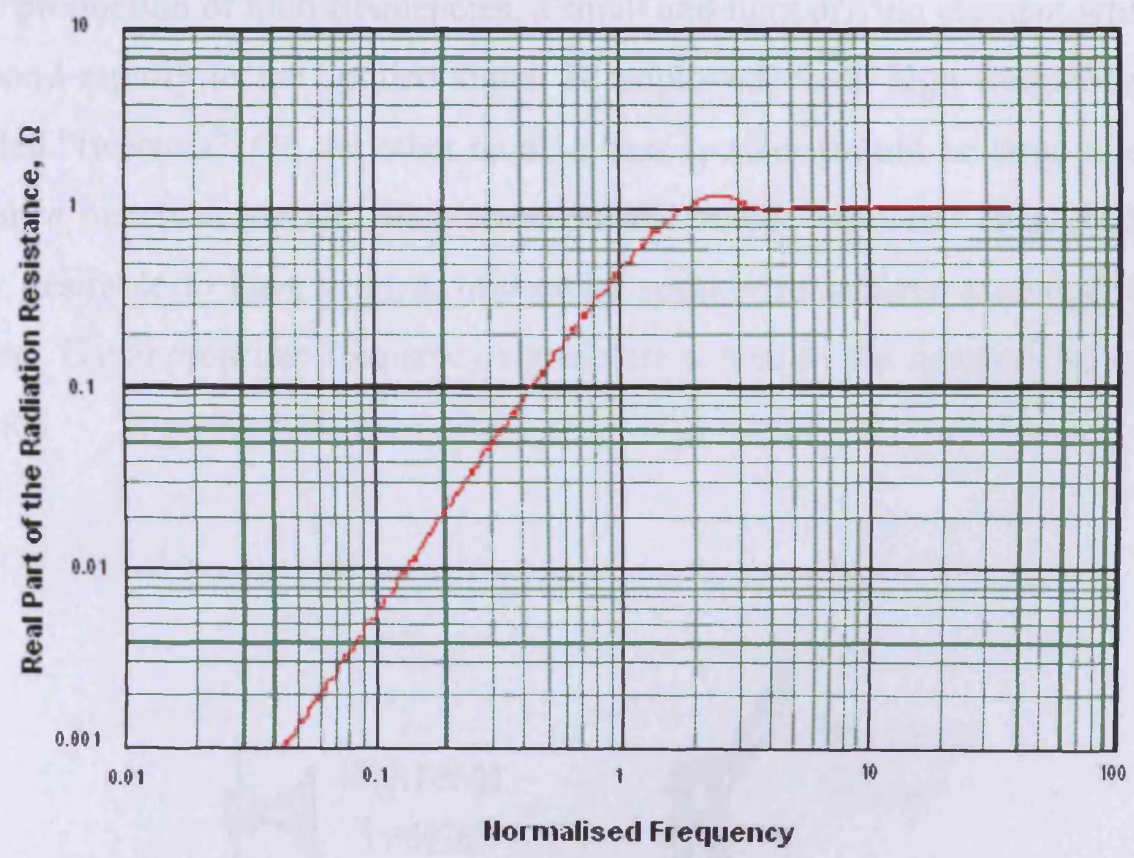

Figure 4.8: Real part of the radiation resistance versus normalised frequency [8]

As frequency continues to rise, though, and the wavelength in air reduces to the point where it becomes comparable with the diaphragm dimensions, a major change occurs. Instead of continuing to rise, the real part of the radiation resistance reaches a limiting value and essentially becomes a constant for all higher frequencies. Because of this the diaphragm's acoustic power output now begins to fall at a rate of $12 \mathrm{~dB}$ per octave 
[8]. This doesn't mean that the on-axis pressure response falls away: what generally happens is that the diaphragm's acoustic output becomes restricted to progressively narrower solid angles. In other words, it becomes directional; it begins to beam. The beaming maintains an on-axis pressure proportional to the acceleration and accounts for the loss of acoustic power. Consequently, the pressure response of a typical cone loudspeaker is limited at high frequency.

An obvious solution is to use a very small diaphragm, but such a diaphragm would have to undergo enormous, impractical excursions to produce the volume displacements necessary at lower frequencies. So loudspeaker designers use two diaphragms: large diaphragms provide the volume displacement necessary to reproduce low frequencies; small diaphragm take over at higher frequencies before the output of the larger units becomes too directional.

For the production of high frequencies, a small and light driving element which is able to respond rapidly to the applied signal is employed. Such high frequency speakers are called "tweeters". On the other hand, a bass speaker should be large to efficiently impedance match to the air. Such speakers are called "woofers" (Fig. 4.9) [6]. It is usually desirable to have a third, mid-range speaker to achieve a smooth frequency response. The appropriate frequency signals are routed to the speakers by a crossover network.
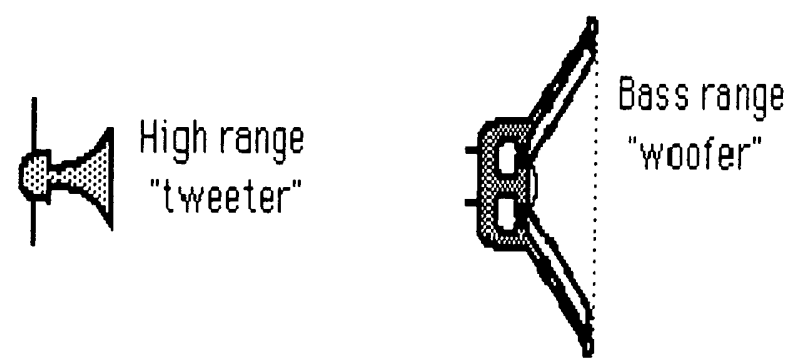

Figure 4.9: Low and high frequency loudspeakers [6] 


\subsubsection{Crossover Network}

Combinations of capacitors, inductors, and resistors direct high frequencies to the tweeter and low frequencies to the woofer. This amounts to filter action. A two-way crossover network divides the frequency range between two speakers whereas a threeway crossover network divides the frequency range between three speakers (Fig. 4.10) [6].

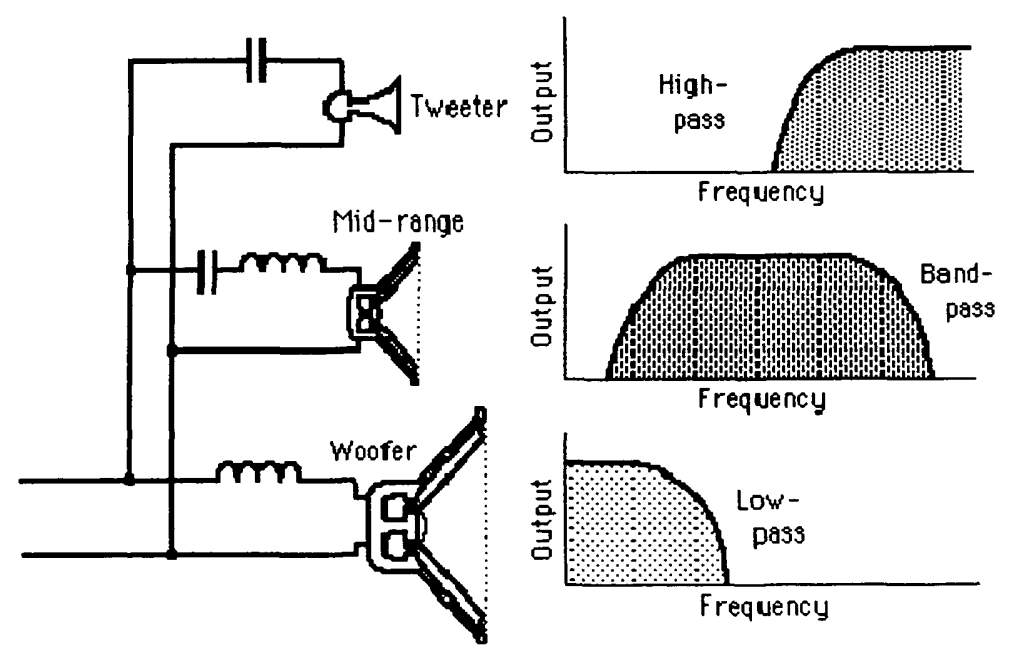

Figure 4.10: Cross-over network for a three-way loudspeaker [6]

\subsection{Flat Panel Loudspeaker}

The operation principle of a conventional loudspeaker is based on the principle of a rigid piston. A stiff and light diaphragm is usually employed to achieve rigid pistonic motion in a conventional loudspeaker. The whole body motion in a conventional loudspeaker has various advantages and is considered to be the most suitable for bass frequency sound reproduction. However, as a result of the same pistonic motion, the sound generated by the conventional loudspeakers becomes increasingly directional for high frequencies. Therefore, in a conventional loudspeaker, cross over circuits and multi-way loudspeakers are usually employed to cover the whole audio frequency range. 
Panel speakers are based on a philosophy contradicting conventional design [8]. The flat panel technology abandons the concept of pistonic motion and considers instead a diaphragm vibrating randomly across its surface rather than coherently. Each small area of the panel vibrates, in effect, independently of its neighbours, rather than in the fixed, coordinated fashion of a pistonic diaphragm. Such a randomly vibrating diaphragm behaves quite differently because power is delivered to the mechanical resistance of the panel, which is constant with frequency [8]. In the case of a flat panel, the radiation resistance is insignificant since the air close to the panel also moves in a random fashion, reducing the effective air load. This means that diaphragm dimensions no longer control directivity and the high frequency output does not become confined to a narrow solid angle about the forward axis.

The technology behind flat panel speakers encourages the diaphragm to produce the maximum number of bending resonances, evenly distributed in frequency. The resulting vibration is so complex that it approximates random motion [8]. Figure 4.11 [8] shows a snapshot of the random diaphragm movement in a flat panel loudspeaker. The random motion of the panel reduces the directivity related problems [8].

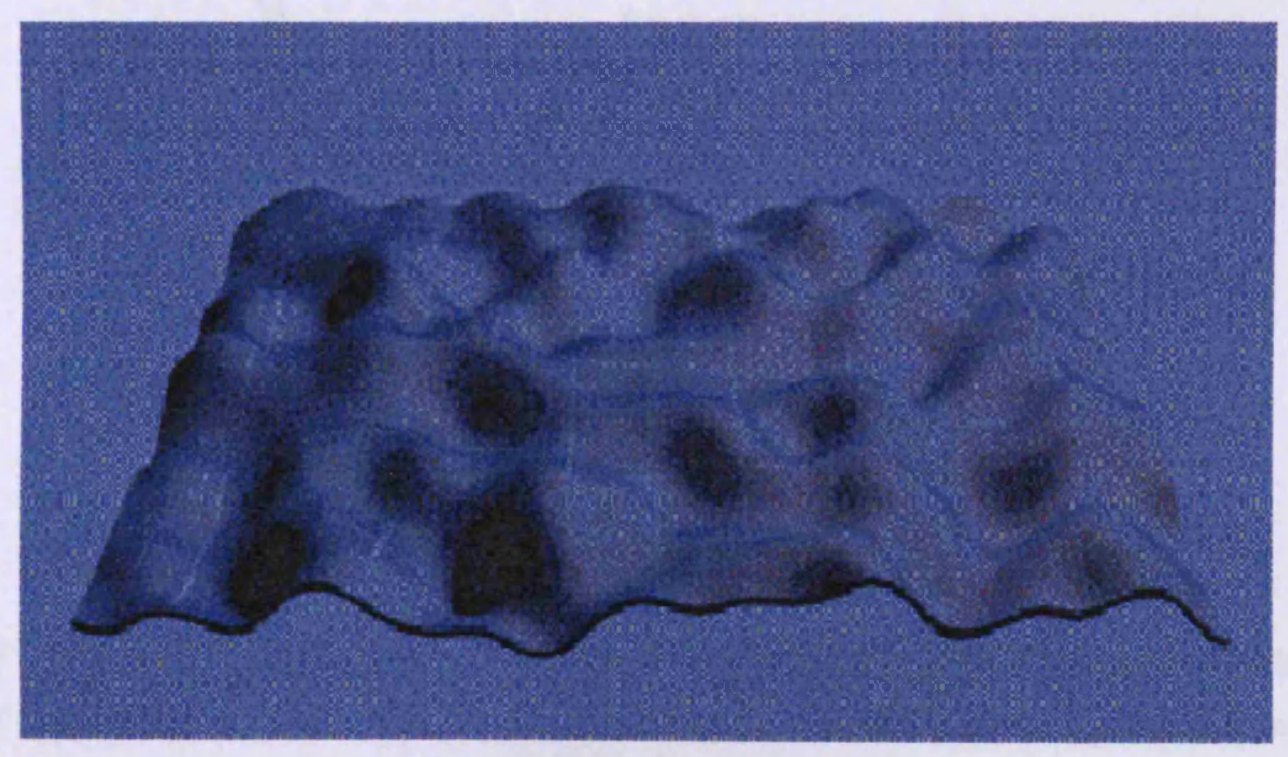

Figure 4.11: A snapshot of the flat panel motion [8] 
A schematic diagram (Fig. 4.12) $[9,10]$ of the flat panel loudspeaker shows the different parts of the panel speaker in details.

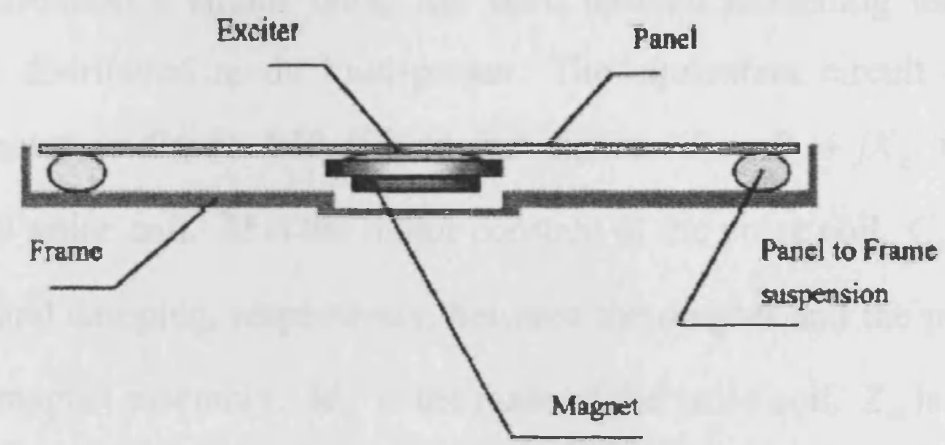

(a)
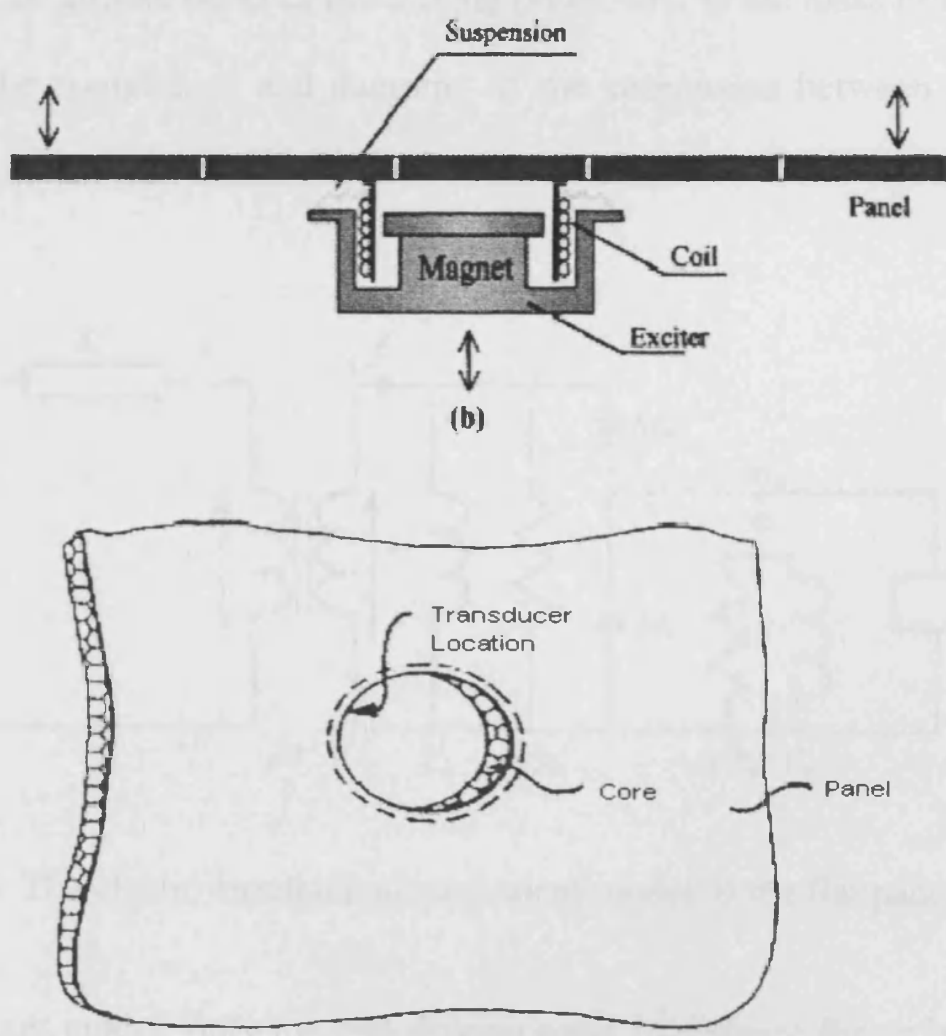

(c)

Figure 4.12: Schematic of a flat panel speaker (a) The panel loudspeaker consisting of a panel and an exciter [9] (b) Details of the inertia exciter [9] (c) The panel is formed from a sandwich material made of two skins and a core material [10]. 


\subsubsection{Electro-Mechanical Model}

An electro-mechanical equivalent circuit, based on Newton's second law, Lorentz force and Kirchhoff's circuit laws, has been derived modelling the panel exciter system of a distributed mode loudspeaker. The equivalent circuit of a flat panel system is shown in figure 4.13 [9]. In this figure, $Z_{c}=R_{c}+j X_{c}$ is the electrical impedance of voice coil. $B l$ is the motor constant of the voice coil. $C_{s}$ and $R_{s}$ are the compliance and damping, respectively, between the magnet and the panel. $M_{m}$ is the mass of the magnet assembly. $M_{c}$ is the mass of the voice coil. $Z_{m}$ is the mechanical impedance of an infinite panel at the driving point. $M_{f}$ is the mass of the frame. $C_{p}$ and $R_{p}$ are the compliance and damping of the suspension between the panel and frame [9].

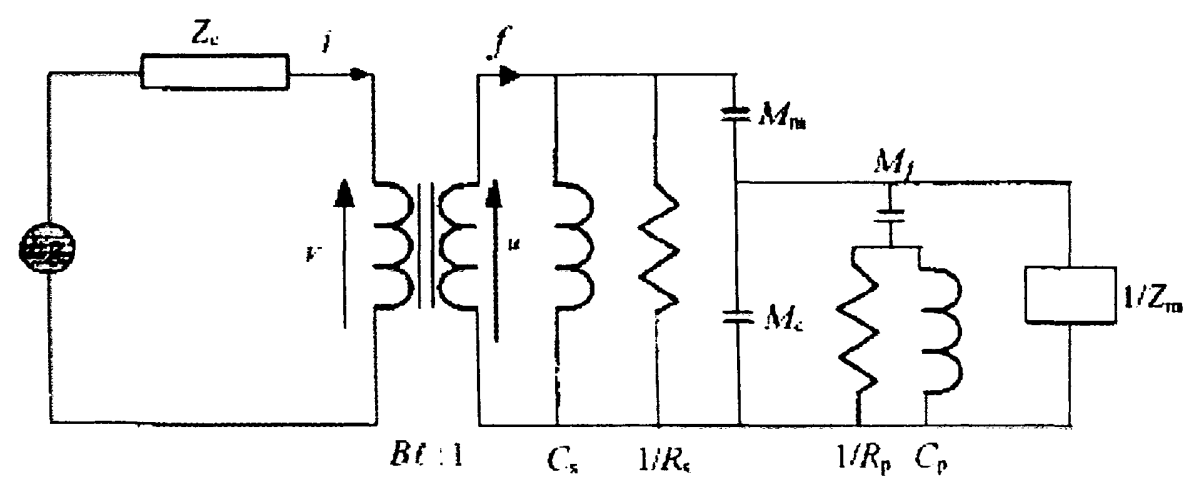

Figure 4.13: The electro-mechanical equivalent model of the flat panel speaker [9]

In the equivalent model, only the real driving point impedance for an infinite plate is used and the radiation loading is neglected. The equivalent circuit can be simplified into a Thevenin equivalent circuit of Fig. 4.14 [9], where $V_{S}$ is the voltage source, $Z_{T}$ is the source impedance reflected to the mechanical side, and $Z_{L}$ is the mechanical impedance of the load including the panel and the exciter assembly. The force is determined with the attached driver assembly taken into account. 


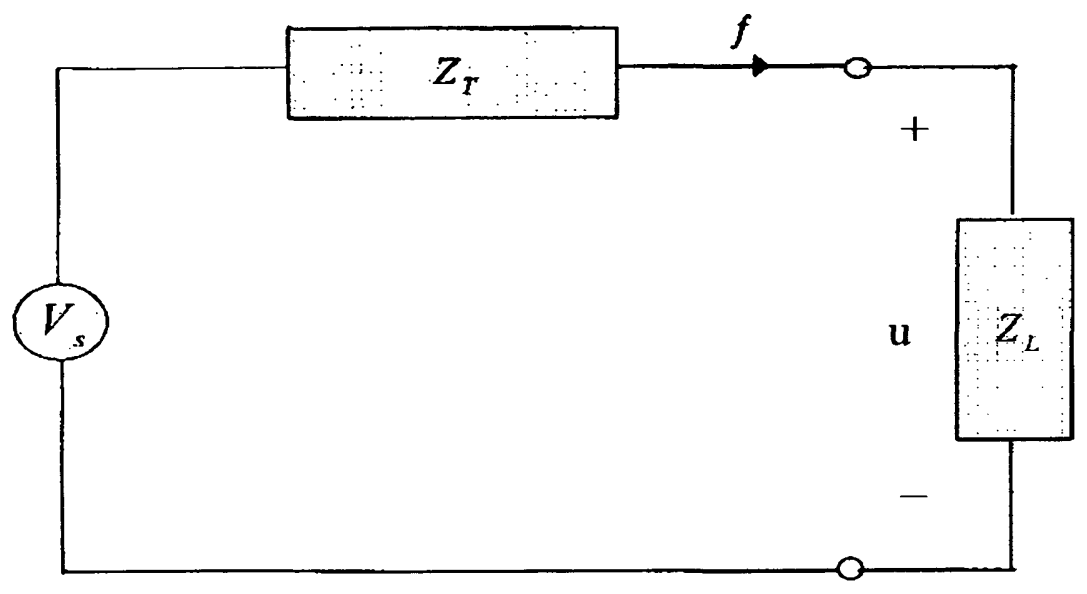

Figure 4.14: The simplified electro-mechanical-acoustical model of the circuit [9]

The power delivered to the load $Z_{L}\left(=R_{L}+j X_{L}\right)$ can be calculated as [9]

$$
W_{L}=\frac{\left|V_{S}\right|^{2} R_{L}}{\left(R_{S}+R_{L}\right)^{2}+\left(X_{S}+X_{L}\right)^{2}}
$$

\subsubsection{Advantages of Distributed-Mode Technology}

The diaphragm of a Distributed-Mode Loudspeaker (DML) vibrates in a complex pattern over its entire surface. The sound field created by this complex pattern of vibration is also complex but a short distance away it takes on the far-field characteristics of the DML radiation. Even when the diaphragm is quiet large relative to the radiated wavelength, the DML approaches omni directionality as it shows directivity of a true point source [10].

At radiated wavelengths, in a conventional loudspeaker, that are small relative to the diaphragm dimensions, interference takes place between the radiation from different regions of the diaphragm. The interference increases the off-axis severity. Therefore, the characteristic radiation pattern exhibits strong beaming (Fig. 4.15). At the other extreme, in a randomly vibration panel, there is a random distribution of diaphragm velocity with respect to magnitude and phase. The disparity in path-length between different areas of the diaphragm and the receiving point is still present, but because there is now no correlation between the source points' output, there is no global 
interference (Fig 4.15) [8]. Hence the radiated sound is dispersed evenly in all directions. Diffuse radiation of high order becomes omnidirectional in the far field [8]. This particular advantage of flat panel sound radiation mechanism bypasses the need for crossover circuits and multi-way high frequency speakers.

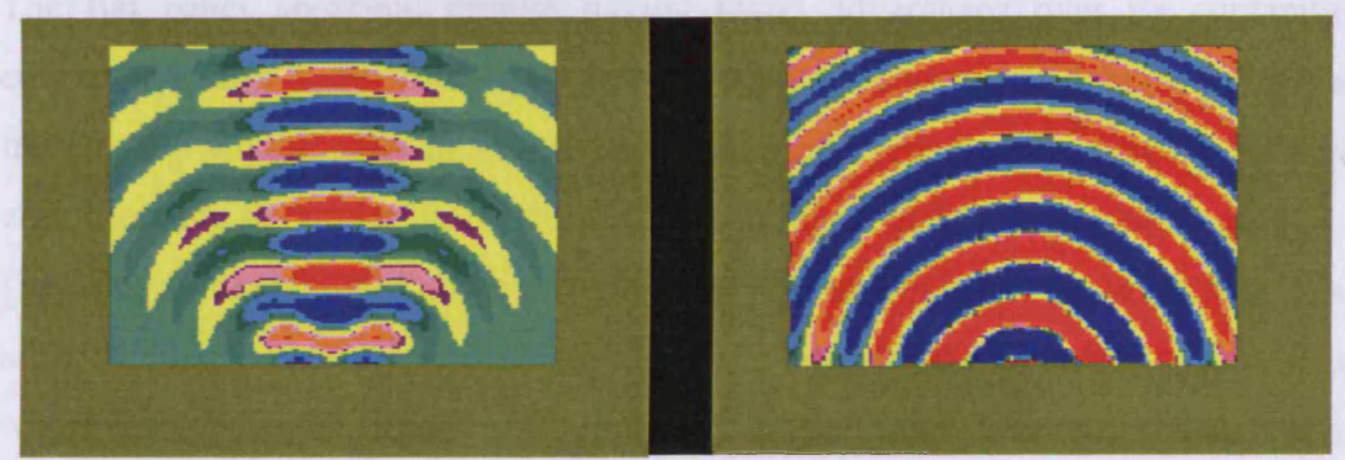

(a)

(b)

Figure 4.15: FE-simulated sound field of (a) an ideal piston loudspeaker (b) a randomly vibrating panel [8].

In a conventional diaphragm, moving mass determines the upper limit of the frequency response. With a flat panel loudspeaker panel there is no equivalent restriction, and therefore the technology is scaleable. Moreover, as claimed by its innovators [8], the panel can be large without directivity or suffering treble response. Increase in panel size results in the frequency of the fundamental bending resonance being lowered, which not only extends the bass response, but also increases modal density in the mid and high frequencies.

Another important advantage of using the flat panel technology is that it omits the need for a special enclosure design. The acoustic output from both sides of a flat panel is useful [8]. The power radiated from the back face sums up constructively with radiated power from the front face of the panel. This is due to the complexity of distributed-mode radiation and the uncorrelated phase of the individual radiating elements as seen from the far-field point of view. 
The other important advantages, the DML speaker provides, over to its conventional counterpart are compactness, linear on-axis, attenuation, insensitivity to room conditions, bi-polar radiation, good linearity, and so forth [8].

\subsubsection{Disadvantages of Distributed-Mode Technology}

The flat panel speakers, despite having many advantages over its conventional counterpart, also suffer from few disadvantages. In a randomly vibrating flat panel, there exists vibrationally most active sub-areas and vibrationally inactive sub-areas, corresponding to "nodes" and "anti-nodes" (or "dead-spots"), respectively (Fig. 4.16) [10]. The combination of nodes and anti-nodes by superposition and clustering at subareas forming regions of substantially more and less vibrational bending wave activity which can be considered as "combines nodes" and "combines dead spots", respectively. Poor acoustic performance in flat panels is influenced by the presence and distribution of the dead-spots and combined dead-spots. Inherently better acoustical performance or action arises from care taken to reduce, preferably as near as practicably eliminate, occurrence of these combined dead-spots [10].

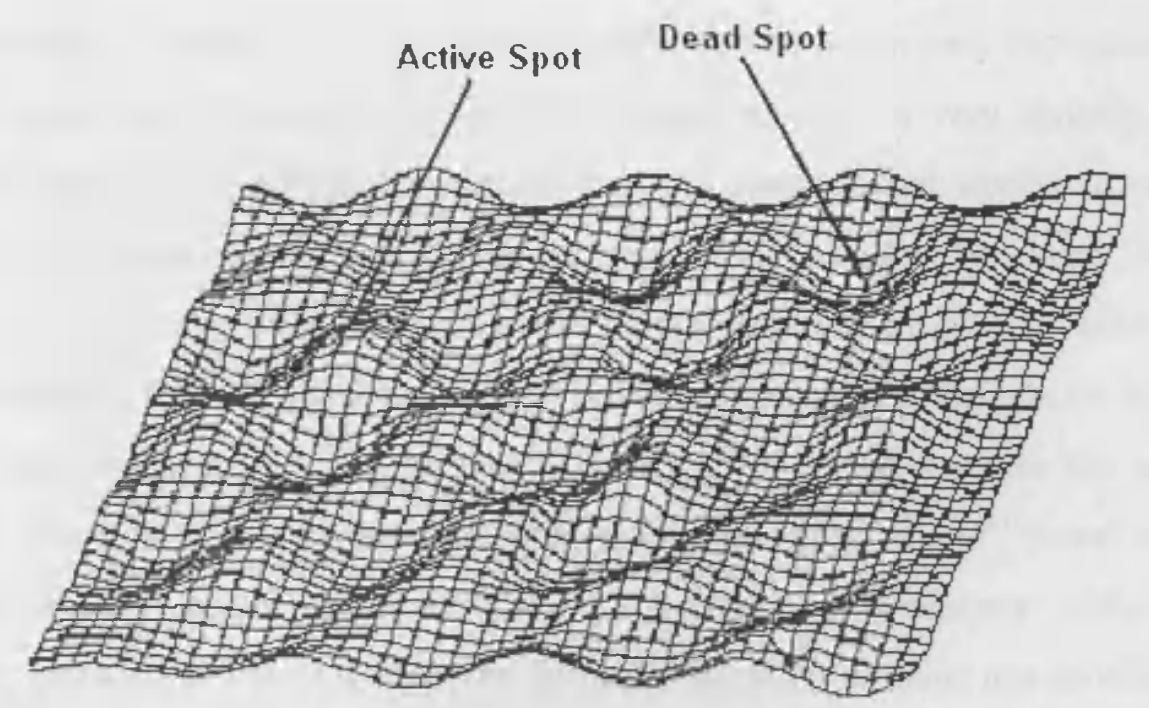

Figure 4.16: "Nodes" and "Anti-Nodes" in the flat panel motion [10]

One of the main limitations of flat panel technology is its impulse response. Because of the independent random vibration of the different parts of the panel, the impulse response of a distributed mode loudspeaker displays a long resonant tail (Fig. 4.17) 
[8]. This feature is particularly a limiting factor for quality sound reproduction in flat panel speaker. The long resonant tail from an impulse response shows that a random vibration, although providing some advantages over its conventional counter part, can distort the quality of a reproduced sound.

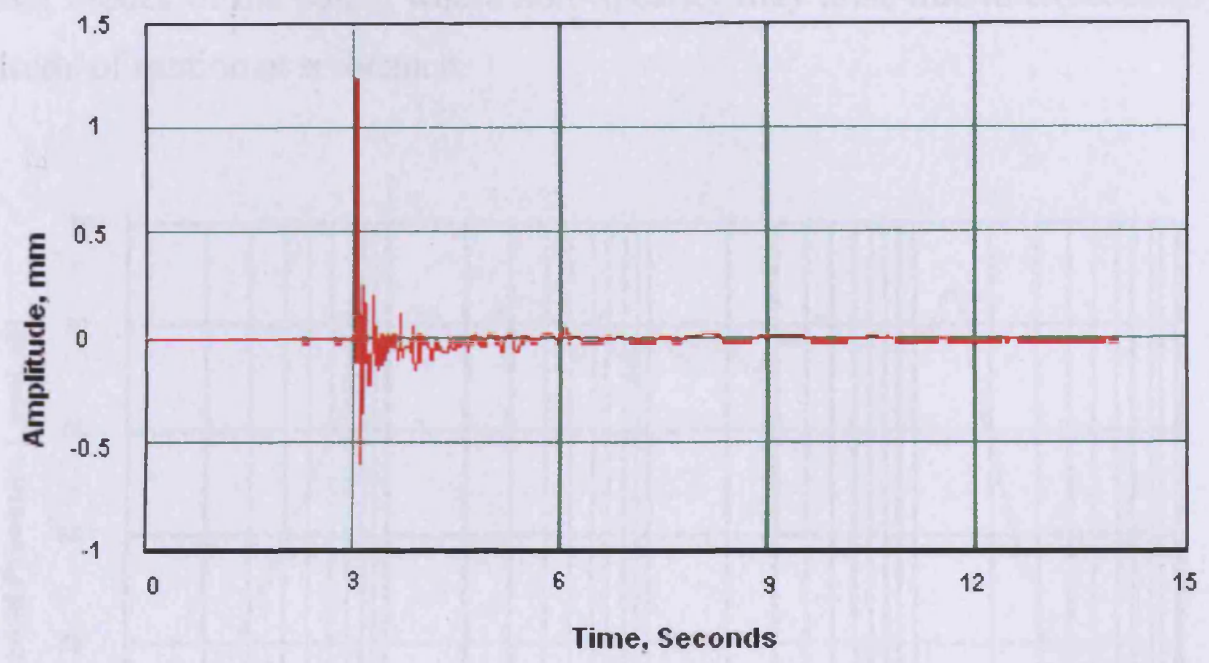

Figure 4.17: Impulse response of a typical flat panel speaker [8]

Another major limitation of a flat panel speaker is that it can only reproduce sound effectively at high frequencies where the resonant modes are very densely packed. The flat panel often needs to be combined with a conventional woofer to cover the lowest two or three octaves in high quality applications. The low frequency limitation (Fig. 4.18) [8] in flat panel speakers could be due to the hydrodynamic short circuit phenomenon: a flexible infinite panel has no acoustic output at frequencies below the coincidence frequency $[9,11]$ at which the speed of sound matches the speed of bending wave in a panel. However, this is not true for a "finite" panel and it is possible to have sound radiation below the coincidence frequency; although the acoustic radiation at low frequency in flat panel speakers remains not as efficient as rigid pistons because of cancellations of volume velocity on the surface [9].

Comparative studies [9] show that the distributed mode loudspeakers have a problem of sensitivity and efficiency in comparison with the conventional speakers. Poor 
radiation efficiency below the coincidence frequency is a physical constraint of flexible panels.

The distributed mode loudspeakers appear to have higher harmonic distortions than the conventional speaker does [9]. A possible explanation is that the DML relies on resonant modes of the panel, where non-linearity may arise due to exceedingly large amplitude of motion at resonance.

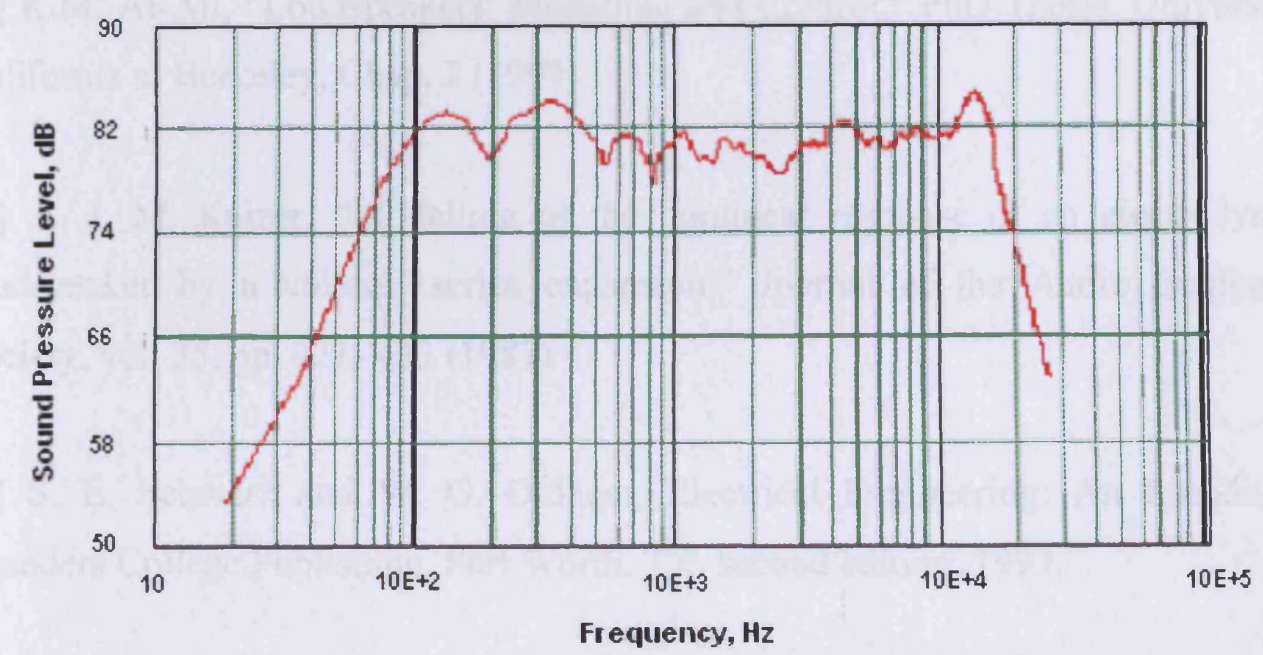

Figure 4.18: Frequency response of a flat panel speaker showing limitation of bass response [8] 


\section{References:}

[1] V. Adam, "Loudspeaker Behaviour Under Incident Sound Fields," PhD Thesis, Federal Polytechnic school of Lausanne, Chap. 1 (2002)

[2] B.E. Anderson, "Derivation of Moving-Coil Loudspeaker Parameters using Plane Wave Tube Techniques," M.S. Thesis, Brigham Young University, Chap. 1 (2003).

[3] K.M. Al-Ali, "Loudspeakers: Modelling and Control," PhD Thesis, University of California at Berkeley, Chap. 2 (1999).

[4] A. J. M. Kaizer, "Modelling of the nonlinear response of an electrodynamic loudspeaker by a volterra series expansion," Journal of the Audio Engineering Society, vol. 35, pp. $421-433$ (1987)

[5] S. E. Schwarz and W. G. Oldham, Electrical Engineering: An Introduction, Saunders College Publishing, Fort Worth, TX, second edition, 1993.

[6] Loudspeaker Hyperphysics -

http://hyperphysics.phy-astr.gsu.edu/Hbase/audio/spk.html

[7] N.J. Harris, M.O.J. Hawksford, " Introduction to distributed mode loudspeakers (DML) with first-order behavioural modelling," IEE Proceedings on Circuits, Devices and Systems, vol. 147, Issue 3, pp. 153-157 (2000).

[8] H. Azima, "NXT Technology," NXT technical paper, 1996.

[9] M. R. Bai, T. Huang " Development of panel loudspeaker system: Design, evaluation and enhancement," J. Acoustical Society of America, vol. 109, Issue 6, pp. 2751-2761 (2001).

[10] New Transducers Ltd., “Acoustic Devices,” patent W0 97/09842, 1997 
[11] M. Heckl, B. A. T. Petersson, L. Cremer, "Structure- Borne Sound: Structural Vibrations and Sound Radiation at Audio Frequencies," Springer, 2005 


\subsection{Introduction}

The loudspeaker industry has accomplished a lot since the first invention took place in terms of sound reproduction techniques and the overall structural details. During the last few decades research has been carried out in both industries and in the academia for the design and development of efficient loudspeakers.

Contributions to the art of loudspeaker design are well documented in the technical literature, but for the most part, only if intended for professional or commercial application. More than eighty years of high fidelity loudspeaker development are known mainly by product reviews in consumer publications and a few landmark products that serve as continuing standards. Countless others have disappeared. The following presents a brief review of what took place in loudspeaker research from the 1920 s to the present.

The basic principle of a dynamic speaker has changed little since it was patented by Ernst Siemens in 1874 (US Patent No. 149,797). Siemens described his invention as a means for obtaining mechanical movement of an electrical coil from electric currents that flowed through it. The original intent of his invention was to move a telegraph arm. Alexander Graham Bell applied the principles of Siemens device to the telephone two years later. Thomas Edison is credited with inventing the loudspeaker as it is known today. It consisted of a flexible diaphragm (cone) attached to the throat of an acoustical horn.

Over the past 80 years the variety of device sold as high fidelity loudspeakers is truly amazing. At present, loudspeakers are used in almost all the places such as cars, houses, halls, educational institutions etc. Since the geometry of each place is different, loudspeakers need to be designed specifically for a given location to meet the optimum performance level. 
There are various research areas in loudspeaker design and development. Developing cheap and powerful magnets, re-design of the electronic circuit, various mathematical modelling methods are to name a few to indicate the extent of which research is going on at the present in the loudspeaker development arena.

The following paragraphs present the extent of various research areas in loudspeaker design and development based on specific subjects and mechanisms. This overview of loudspeaker research has taken into account the interesting designs than have emerged over the last few decades and changed the loudspeaker industry. The review of loudspeaker research gives an insight into the rapid changes and proliferation of new ideas that characterized consumer loudspeaker development from the beginning of loudspeaker development to the present. Researchers over the years, with a profusion of different approaches, have all tried to achieve an accurate sound reproduction. But the perfect loudspeaker, till today, remains a challenge and the theorists, engineers, and loudspeaker researchers continue to pursue that goal.

\subsection{Transducer Design}

Rice and Kellogg [1] described the first fully realized moving-coil direct-radiator electrodynamic loudspeaker (Fig. 5.1) [1] in 1925. The paper described a series of tests directed to the evolution of a loudspeaker, free from resonance. Rice and Kellogg claimed that it is possible to make an ideal sound reproducer on the principle of a small and light diaphragm. It was shown on theoretical grounds that a small diaphragm, the motion of which is controlled by inertia only, and located in an opening in a large flat wall, would give an output sound pressure proportional to the actuating force, independent of frequency. The best acoustic responses were obtained with diaphragms that are so flexible that their resonance was below the lowest important acoustic frequency. The novel loudspeaker developed by Rice and Kellogg, as compared with ordinary loudspeakers, radiated much more of the low tones and more of the very high frequencies which makes for clearer articulation. However, the extension of the range of response of the loudspeaker to higher and lower frequencies introduced defects in the remainder of the system more noticeable, particularly roughness and blasting due to overworked amplifiers. Therefore, Rice and Kellogg 
suggested that it is important that the amplifier used with the new loudspeaker be designed to have ample capacity.

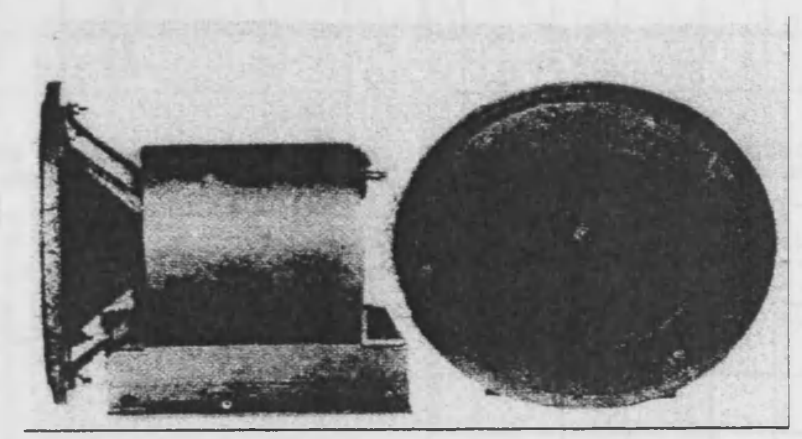

Figure 5.1: First practical commercial moving-coil direct-radiator loudspeaker design, utilizing a conical paper diaphragm [1]

Olson [2] in 1938 made an important observation after experimenting with various types of loudspeakers: the double coil, single cone loudspeaker; the single coil, multiple cone loudspeaker, the double coil, double cone loudspeaker and the multiple coils, multiple cone loudspeaker. He observed that, for a loudspeaker to obtain uniform response, relatively high efficiency and adequate power handling capacity over a wide range requires a large diameter, rugged diaphragm and heavy coil at the lower frequencies and a relatively light weight vibrating system at the higher frequencies. This is a very important observation as far as loudspeaker response is concerned. The idea of different types of vibration systems for effective reproduction of various frequencies eventually led to the evolution of cross-over systems.

Olson, Preston and May [3] reported on the developments in direct radiator highfidelity loudspeakers in 1954. These included methods of improving loudspeaker response through adjustments to the cone, surround, enclosure shape and mounting. Practical realizations included the famous LC1A 3810 millimetre "duo-cone" coaxial loudspeaker, which utilized small conical domes attached to the main low frequency cone to improve dispersion (Fig. 5.2) [3]. The effect of these domes was analyzed using a ray-acoustics graphical analysis method described as "reflected, diffracted, and radiated pencils of sound." 

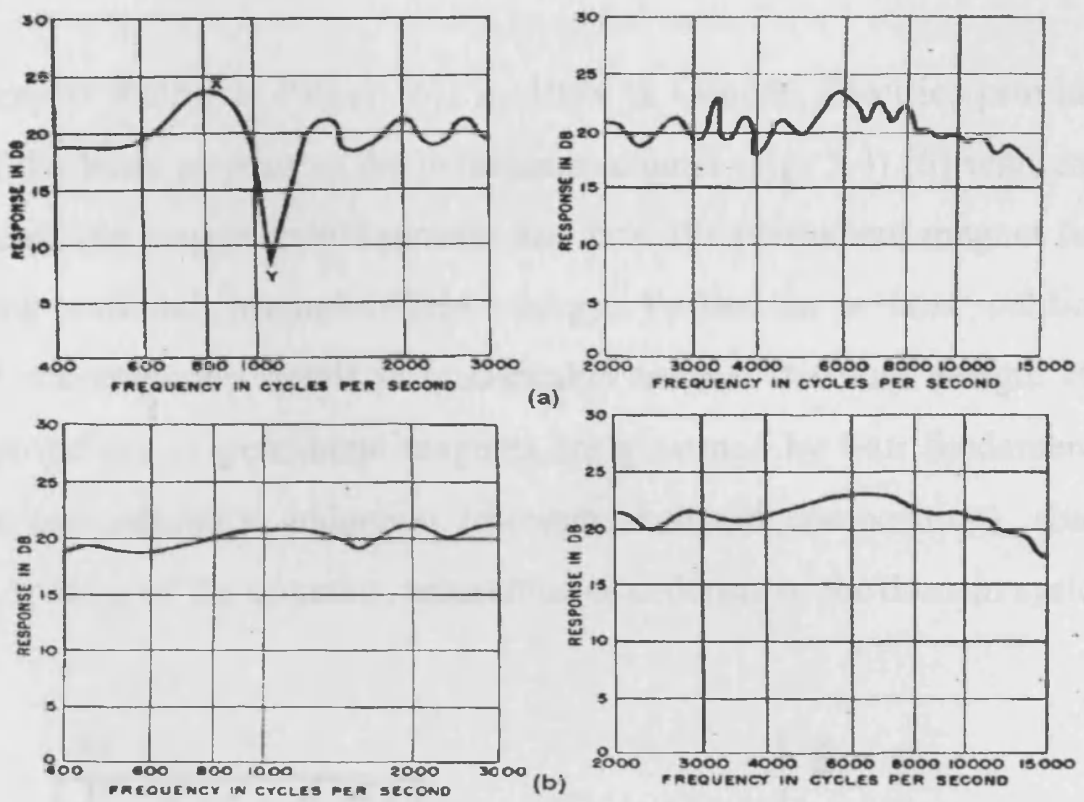

Figure 5.2: Mid and high frequency response of a "duo-cone" coaxial loudspeaker (a) with a conventional suspension system (b) with a suspension system equipped with a rubber damper [3]

Cohen [4] and Fiala [5] described the basic mechanics of loudspeakers, including cone performance requirements and moving system practicalities (Fig. 5.3) [4]. Cohen discussed the unusual stresses to which the diaphragm of a loudspeaker is subjected to while reproducing sound and the need for a precision built diaphragm for optimum mechanical strength. He concluded that good loudspeaker design was very much a matter of good mechanical design. Fiala, on the other hand, discussed the low frequency loudspeaker design parameters. Fiala suggested that, in order to achieve low frequency response, the cone had to move with large amplitudes proportional to the driving current in the voice coil. This can be expressed simply by saying that the whole system should have a linear displacement versus current relationship. In detail this requires that the voice coil stay in a uniform magnetic field for the whole length of travel and that the suspension system be linear and symmetric for the maximum amplitude.

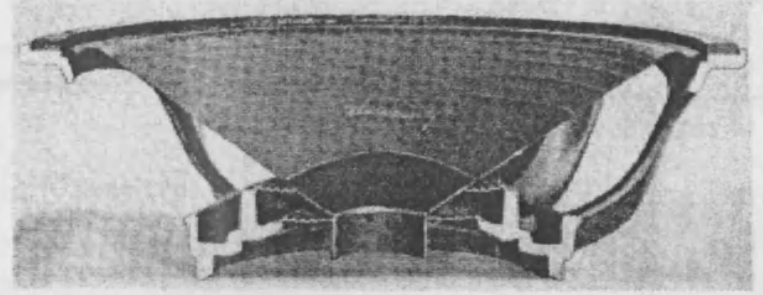

Figure 5.3: Girder basket construction and double spider arrangement [4] 
Magnet expert Rollin J. Parker [6], in 1958 at General Electric, provided a brief review of the basic physics of the permanent magnet (Fig. 5.4) [6] with emphasis on the nature of the magnetization process and how the permanent magnet functions in establishing external magnetic field energy. Parker, in a later publication [7], described contemporary trends in loudspeaker magnet structure design. He claimed that the properties of permanent magnets are governed by four fundamental factors and these are saturation induction (through chemical composition), shape of the domains, spacing of the domains, orientation or ordering of the domain system.

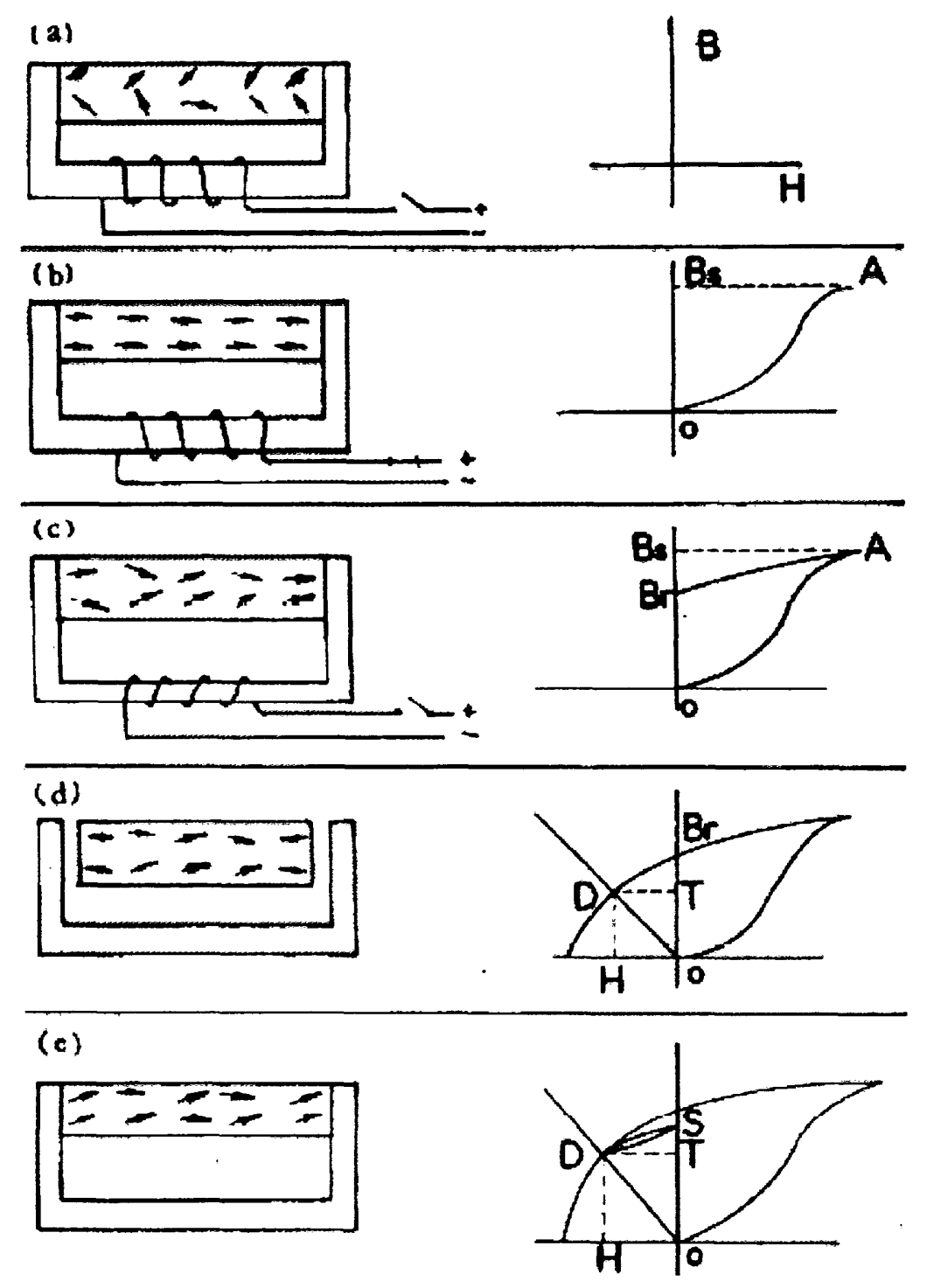

Figure 5.4: Pictorial Description of the Basics Physics of the Permanent magnet [6] 
King [8], in 1969, discussed the current trends in loudspeaker voice coils. He described in detail the moving coil loudspeaker design parameters: matching and control of impedance, efficiency calculation, coil length, coil materials, power handling, demagnetization effects and others. The discussion in this paper showed that the design of a loudspeaker voice coil involved many considerations, and that many of the design problems were best approached on an empirical basis. King suggested that computer programming of basic design criteria and empirical data would enable a more systematic approach to design requirements.

In 1977 Gilliom et al. [9] presented a broad discussion of the design problems in highlevel cone loudspeakers. The paper considered the problem associated with high acoustic power and the consequent reduction in efficiency. High acoustic power has usually been achieved through the use of heavy voice coils, cones, and suspension parts and through large air-gap clearances but all these measures have resulted in reduced efficiency. Gilliom proved, by using mathematical relations, loudspeaker designs which require a large moving mass can be made to have high reference efficiency by increasing the $B l$ product as much as necessary, but bandwidth will be reduced at both ends of the frequency range. Conversely, maintaining a given lowfrequency response forces a reduction of reference efficiency as mass is increased (Fig. 5.5) [9].
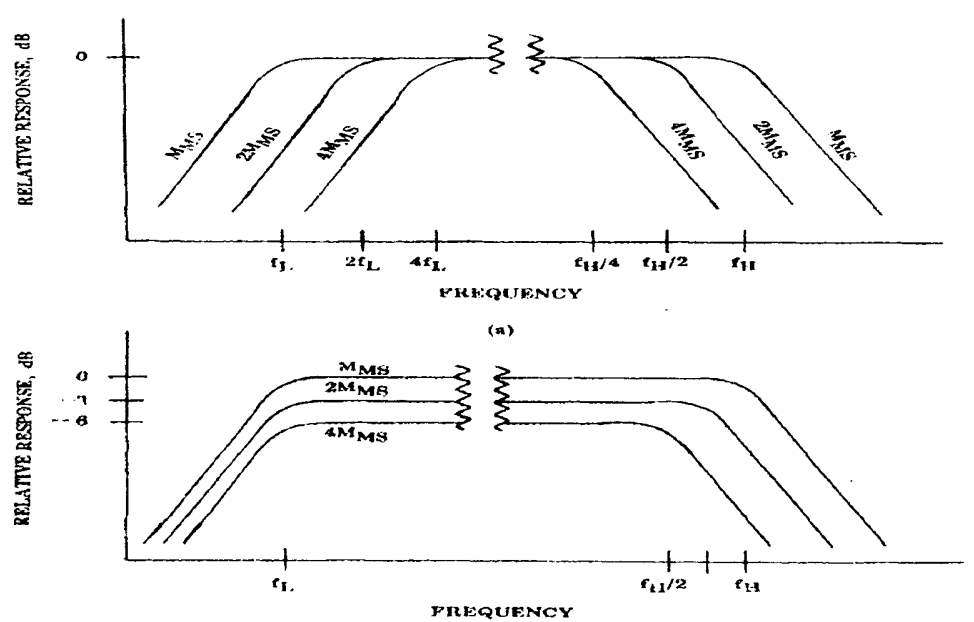

(b)

Figure 5.5: Relative response versus frequency for families of idealized loudspeakers,

(a) Reference efficiency is held constant while moving mass is varied, (b) Lower corner frequency is held constant while mass is varied. In each case the value of the $B l$ product was adjusted to provide a uniform low-frequency [9] 
Bottenberg, Melillo, and Raj [10] in 1980 discussed the dependence of loudspeaker parameters on the properties of magnetic fluids. Magnetic fluids (ferrofluids) have been employed since 1974 as an integral component in loudspeaker production. The intense magnetic field in the air gap retains the fluid in intimate contact with the poles and voice coil such that it increases heat transfer from the voice coil, aids in the assembly of the voice-coil structure into the driver by providing centering forces and contributes fluid-mechanical damping. This paper presented experimental data which demonstrated the ability of ferrofluid to effectively conduct heat from the voice coil to the surrounding pole structure. Voice coils immersed in ferrofluid were shown to have significantly lower temperatures for a given applied power input for a specific time, as compared to the identical coil in a simple air gap. It was also demonstrated that the time required for the voice coil to return to the heat sink temperature was significantly lower when ferrofluid was present.

The mass and the specific geometry of the magnet structures are significant in the application of loudspeaker or driver. Newman and Fidlin [11] at Electro-voice reported an exciting new magnetic material for loudspeaker development (Fig. 5.6) [11] in 1989. This paper proposed the use of a neodymium based magnet structure for high-performance compression driver. The goal of their experiment was to replace the ferrite magnetic structure of a state-of-the-art compression driver with a $\mathrm{NdFeB}$ structure. The results from the experiment indicated that the most important advantage of using a high energy magnetic material such as Neodymium-Iron-Boron is probably the reduction in weight. The introduction of a high energy product $(\mathrm{NdFeB})$ in the magnetic structure of a compression driver resulted in the reduction of weight by a factor of over 3 while maintaining the same level of acoustic efficiency.

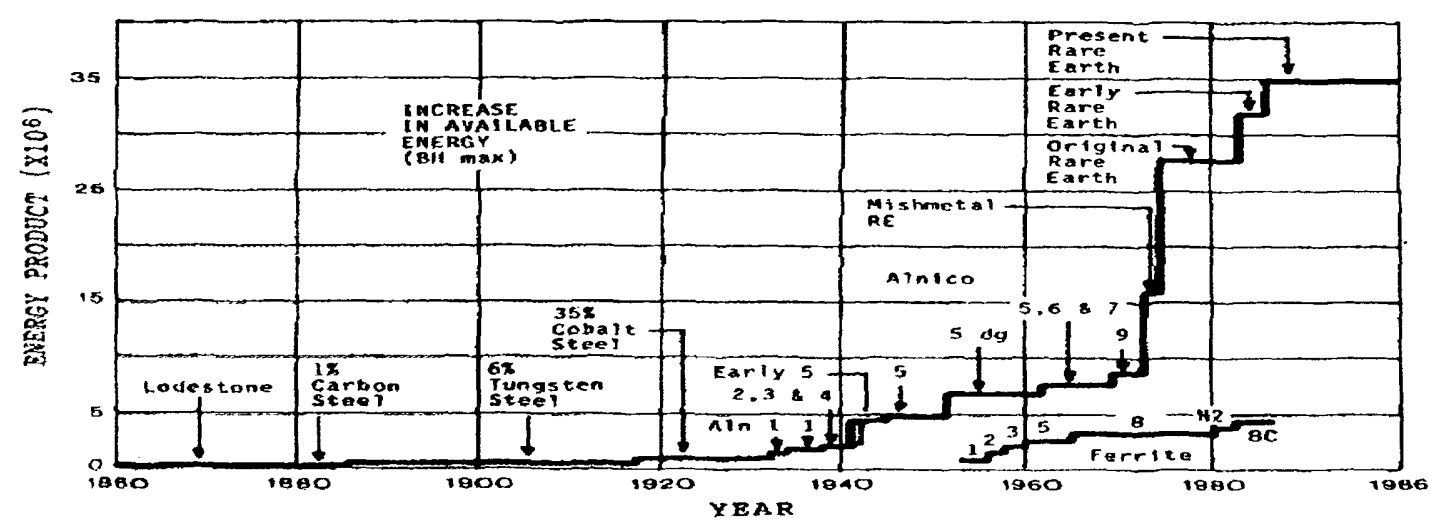

Figure 5.6: Comparison chart for various magnetic materials [11] 
In 1998 Button and Gander [12] described new motor structures and magnetic assemblies for improved loudspeaker performance. This paper described different design options in magnetic materials, magnetic circuit geometries, and voice coil topologies. The novel design proposed in this paper involved two coils that are opposite in phase and reside in oppositely polarized magnetic gaps, thus providing a Lorentz force in the same axial direction. Experimental data showed that the new design delivered more than $3 \mathrm{~dB}$ greater maximum acoustic output over a single gap design. Moreover, when realized using neodymium as a magnet material and properly nesting the structure in a well designed heat sink, the design could yield a significant reduction in weight, lower distortion, lower power compression, and lower inductance than a traditional single gap design and yet maintain cost competitiveness.

Wright $[13,14]$ generated an empirical model for loudspeaker motor impedance. For the idealized loudspeaker, the motor impedance would be a simple inductor. In this paper, Wright showed that the flow of eddy currents in the proximity of the voice coil and in the pole structure causes a significant deviation from the ideal. The general effect of these eddy currents is to increase the motor resistance of a loudspeaker with increasing frequency and to cause non-ideal behaviour of the inductor formed by the voice coil (that is, the interaction of windings) and the pole structure. The pole effects are dominant but the eddy currents within the coil itself are significant as shown by figure 5.7 and $5.8[13]$.
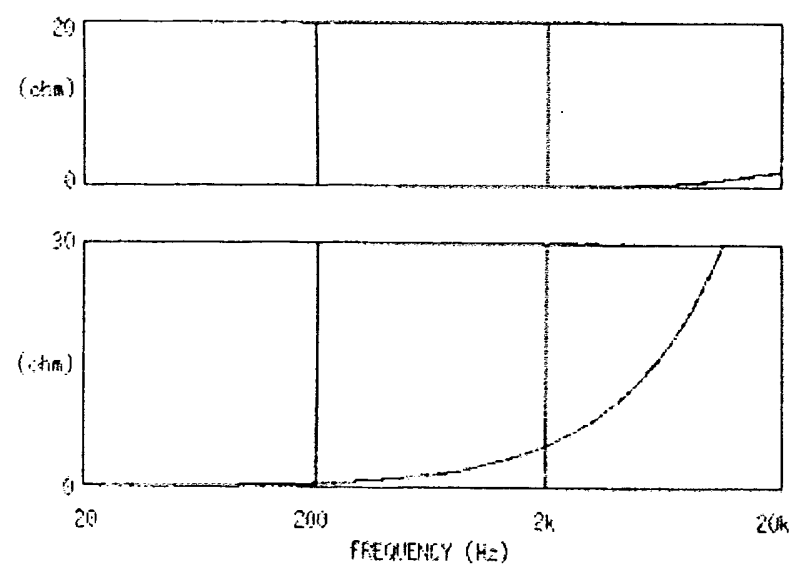

Figure 5.7: Motor resistance (top) and reactance (bottom) curves for air-cored voice coil [13] 

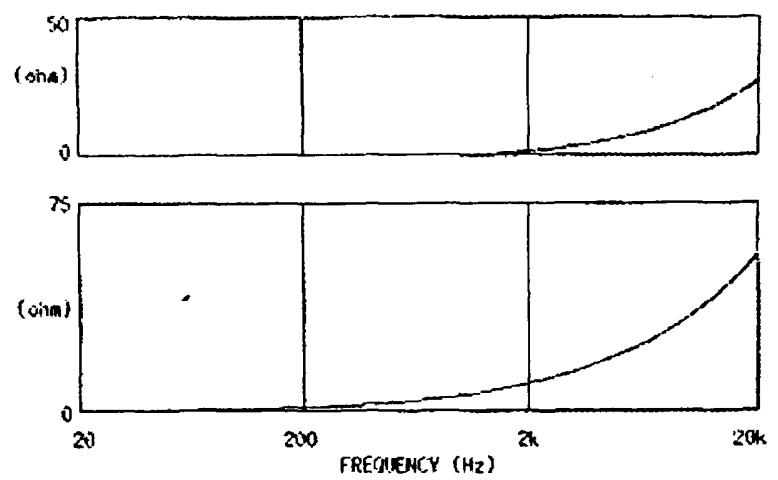

Figure 5.8: Motor resistance (top) and reactance (bottom) curves for coil of Fig. 5.7 located on its pole piece [13]

In 1997 Zuccatti [15] considered optimizing the voice coil airgap geometry for maximum loudspeaker motor strength. The paper proved that a moderately overhung coil, although increased voice-coil resistance more than the $B l$ product, gave a better motor strength than an equal height coil-gap geometry.

The force factor $B l$ plays a very important role in loudspeaker design. It determines the efficiency, the impedance, the SPL response, the temporal response, the weight and the cost. Vanderkooy [16] showed the consequences due to a dramatic increase in the motor strength $B l$ of a loudspeaker driver. High $B l$ values greatly increases the efficiency of the loudspeaker and amplifier and also have a positive influence on other aspects of loudspeaker systems. Box volume can be reduced significantly and other parameters can be altered. Vanderkooy studied prototype driver unit which performed well in a small sealed box. Vented systems, however, do not benefit as much from high Bl. R.M. Aarts [17], in 2005, described a new low-Bl driver that has been developed which, together with some additional electronics, yields a low-cost, lightweight, flat, and very high-efficiency loudspeaker system for low frequency sound reproduction.

Thermal effects in transducers have drawn more attention and understanding as the race for higher power handling and acoustic output continues. In 1986, Gander [18] described dynamic linearity and power compression in moving-coil loudspeakers. At higher input levels, loudspeakers suffer from loss of power efficiency due to rise in voice coil resistance. Gander explored the linearity of power transfer by increasing the 
excitation level at various frequencies. He suggested that better power transfer, both acoustically and thermally, can reduce the linearity problem. Henricksen [19] presented the analysis, measurement, and design of heat-transfer mechanisms in loudspeaker (Fig. 5.9) [19]. He described the need for a specially designed magnetic assembly in which the voice coil can also transfer the heat to the ambient air through the magnet. Therefore the transducer design should consider effective heat transfer mechanism in order to obtain a linear power transfer characteristics.

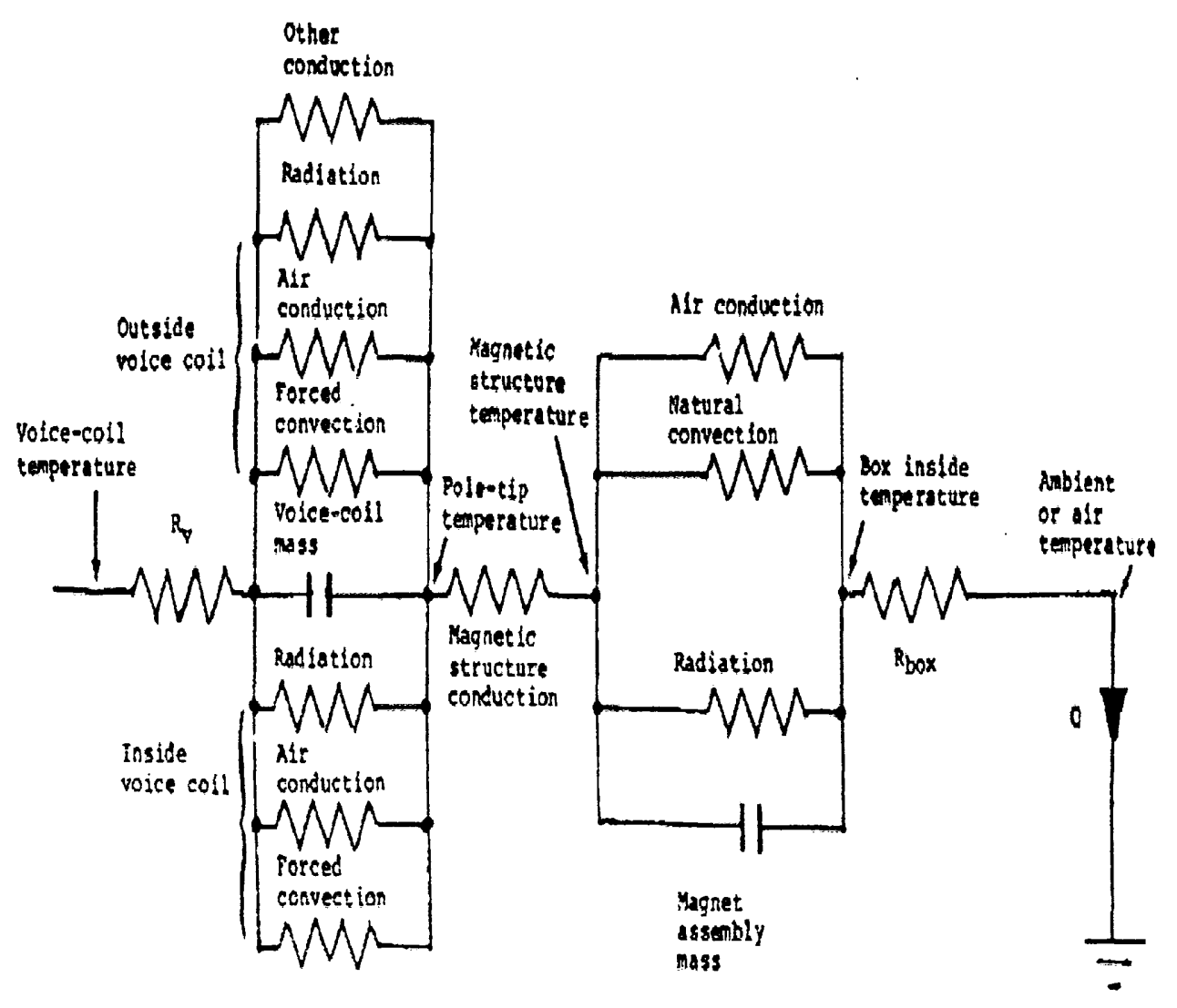

Figure 5.9: Heat transfer mechanism in loudspeaker transducer [19]

Button [20] continued the work on analyzing heat dissipation and power compression in loudspeakers. His paper described the popular voice coil magnetic gap configuration (Fig. 5.10) [20] and suggested the most useful designs that can help dissipate heat effectively. Button concluded that the most effective solution to power compression at higher input is the development of transducers with stationary coils that are directly heat sinked. However, this solution is not cost effective and loudspeakers will also exhibit power compression at the limits of their power 
capacity. Therefore a properly heat sinked loudspeaker driven well below its power capacity will deliver the desired performance.
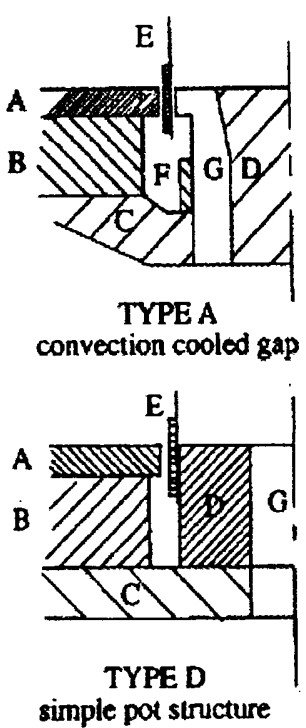

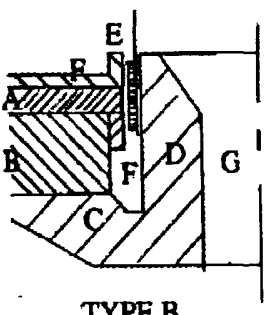

TYPE B
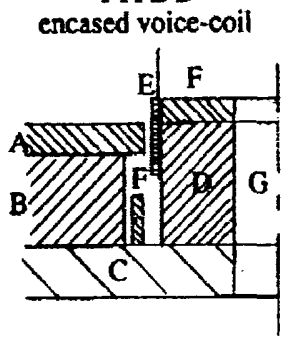

Modified TYPE D lower distortion version
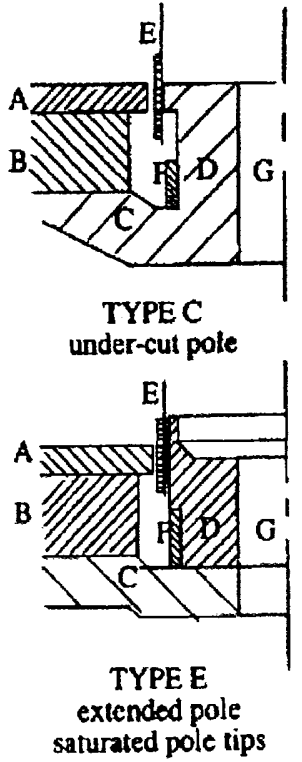

Figure 5.10: Voice-coil magnetic gap configurations. A--top plate, steel; B--magnet, ceramic; C--backplate, steel; D--pole piece, steel; E--voice-coil, aluminium or copper; F--bucking rings for flux modulation reduction and inductance control, aluminium; G-vent (type A has three at periphery of pole piece) [20]

\subsection{Diaphragm and Cone Materials}

From the invention of the first telephone receiving transducer units and cone loudspeakers, the search has continued for the optimum materials to employ as diaphragms to vibrate the air. Barlow [21], in 1970, described a sandwich-construction diaphragm. The diaphragm of the conventional moving-coil loudspeaker was usually made of moulded paper. It was well known that under normal conditions of use, the moulded paper diaphragm was far from rigid, and only behaved as a rigid piston at low frequencies. Therefore Barlow suggested a sandwich construction diaphragm of intense rigidity that produced pistonic motion over a wide range of audio frequency. However, although a large rigid diaphragm did serve effectively at the low frequencies in Barlow's investigation, it probably 
became too directional for high frequencies. Therefore his theory was only valid for a bass range speaker.

In 1978, Frankort [22] presented a summary of his extensive work on the vibration patterns and radiation behaviour of cones, which took advantage of both computer modelling and holographic observation (Fig. 5.11) [22]. In the ideal case the sound radiation from a loudspeaker would have the same amplitude at all frequencies, and the frequency response would be linear. But in reality the overall frequency response is not linear since the loudspeaker cone vibrates as a rigid body only at low frequencies and it is not stiff enough to withstand the inertial forces that occur at higher frequencies and therefore it starts to vibrate in parts and the cone is said to "break up." At higher frequencies, where the depth of the cone is no longer negligible compared with the wavelength, or may even be greater than the wavelength, the radiation deviates from that of the flat piston. The radiation from different parts of the cone then arrives at the point of observation with appreciably different phases, even when the point is on the axis of the loudspeaker. This results in a lower sound pressure at that point.

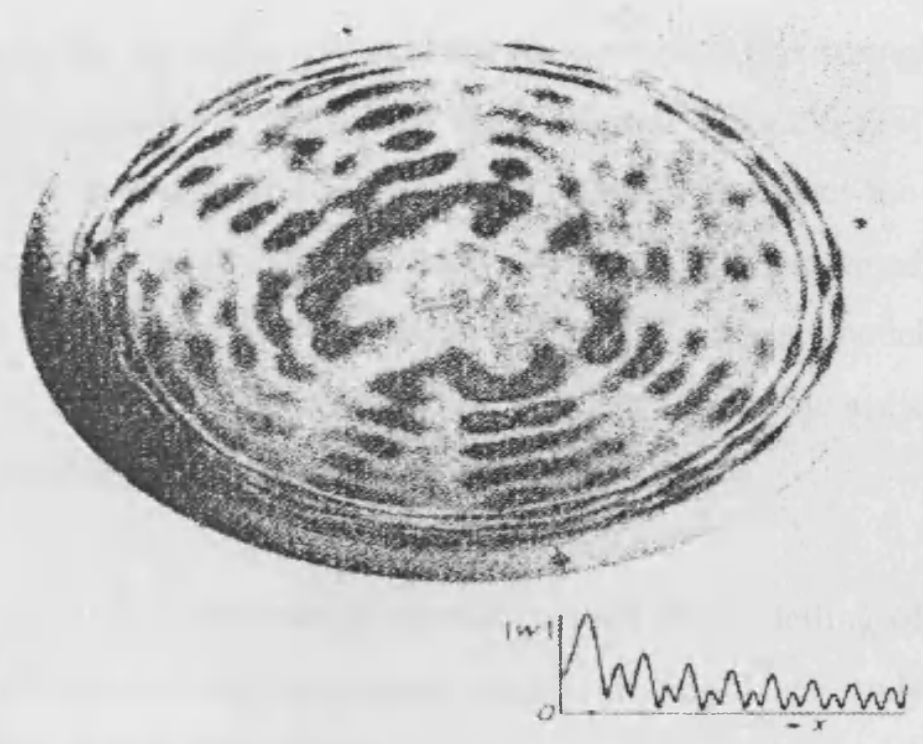

Figure 5.11: Vibration pattern of a loudspeaker with paper cone, made visible by holography at $5929 \mathrm{~Hz}$. The calculated amplitude curve is also shown. ( $\mathrm{x}=0$ at the inner edge of the cone) [22] 
Traditionally, loudspeaker cones were designed in a trial-and-error fashion. Because of complex difficulties encountered in loudspeaker cone design, no mathematical approach had been thought useful until the introduction of the finite-element method. In the 80's, K. Suzuki and Nomoto [23] and Kaizer and Leeuwestein [24] utilized finite-element modelling method to design loudspeakers. This method has been applied to the simulation of cone vibrations and to the sound radiation from loudspeaker cones. The finite-element method permitted the observation of vibration modes, sound-pressure level, sound power, strain and stress of a loudspeaker cone. Struck [25] presented an experimental method called modal analysis as an alternative to finite-element method. The modal analysis allowed a model to be developed from actual measurements. In this investigation, previous problems in the measurement technique were overcome by the use of a non-contacting laser transducer. Using the modal model, Struck simulated structural modifications and studied the dynamic system response. Special application software was used for the measurement, analysis and simulation.

Shindo et al. [26] used finite element method along with experimental results to explore the effect of the voice coil and surround on cone vibration and response. The results showed that the convex cone was strongly affected by the surround, yet virtually unaffected by the voice coil, and the concave cone was strongly affected by the voice coil, but virtually unaffected by the surround. Dobrucki [27] extended the Shindo work by presenting a graphical method of investigation of the surround and voice-coil influence on cones. Shepherd and Alfredson [28] presented an improved computer model of direct-radiator loudspeakers focusing on cone modelling, utilizing the finite-element method coupled to analytical models of the acoustic environment and electromechanical voice coil.

H. Suzuki and Tichy [29] performed extensive computer modelling of the radiation and diffraction effects by both convex and concave domes. Their study showed that, although both the concave and convex loudspeakers vibrate like a piston, the concave dome has a wider peak due to the cavity resonance resulting in higher radiation efficiency. The convex dome, on the other hand, has lower on-axis pressure response in the same region due to the dispersion of energy to the off-axis direction. 
Barlow et al. [30] further described and modelled resonances for various types of loudspeaker diaphragms. He suggested a sandwich construction for increased stiffness and reported torsional resonance not previously suspected in loudspeaker diaphragms. It is not clear; however, from this paper whether the torsional resonance reported here does have any effect on the frequency response or the transient response of a loudspeaker.

In 1978, K. Ishiwatari, N. Sakamoto et al. [31] described the use of boron for highfrequency domes. Sakamoto et al. at a later publication [32] described the construction of a honeycomb disk diaphragm. The investigation employed finite element modelling to model the resonance behaviour of the new diaphragm. This paper claimed that a honeycomb disk diaphragm acts as a rigid plate with piston motion and achieves fiat response and very low distortion. In the same year as the last publication, Niguchi et al. [33] described a reinforced olefin polymer diaphragm that could offer flexural rigidity three times as great compared to conventional cone paper diaphragm (Fig. 5.12) [33]. This paper also claimed that with the new material, it was possible to mould loudspeakers from woofer diaphragms to tweeter diaphragms quickly and accurately and achieve a wide frequency response and low distortion.

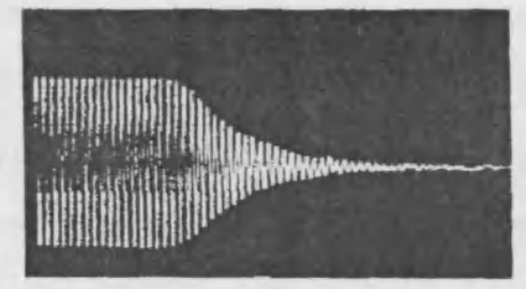

(a)

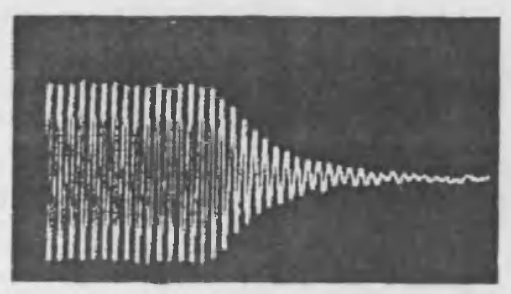

(c)

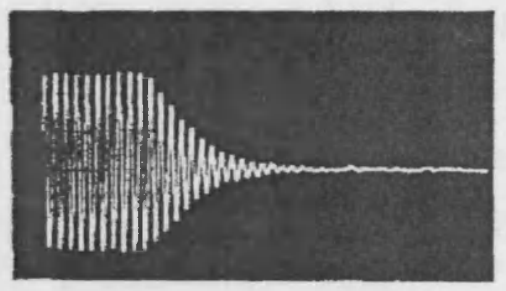

(b)

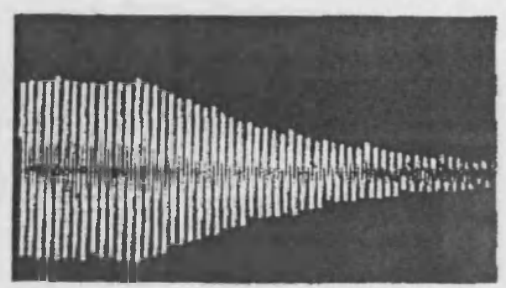

(d)

Figure 5.12: Decay patterns of free vibration. (a) Reinforced olefin polymer diaphragm. (b) Olefin TC diaphragm. (c) Paper cone diaphragm. (d) Aluminium diaphragm [33] 
In 1980, Yamamoto et al. [34] reported on their development of boronized titanium diaphragms and Tsukagoshi et al. [35] at a year later reported on a novel polymergraphite diaphragm. Takahashi et al. [36] reported their development of glass-fiber and graphite-flake reinforced polyimide composite diaphragms and Taguchi et al. in 1986 [37] described a sandwich-construction diaphragm with foamed high-polymer and carbon fiber.

\subsection{Tweeter Design}

The special requirements of transducers designed to reproduce the shorter highfrequency wavelengths were discussed in detail by Sioles [38] in 1956. This paper covered the general constructional and design characteristics of various types of tweeters and elaborated on the fundamental design problems of the moving-coil, hornloaded tweeter. Performance, reliability, and cost considerations were discussed for different types of tweeters. The paper concluded that a horn tweeter performs better than a direct radiator one because of its high efficiency and high output-power capabilities.

Nakajima et al. [39] reported a novel tweeter that has new magnetic circuit composed of small ferrite magnet and magnetic material. The width of the diaphragm was not restricted by magnetic gap and the driving force was constructed so that all surfaces on the diaphragm vibrate in phase. This tweeter had a flat and wide frequency characteristic which covered from $2 \mathrm{kHz}$ to a frequency higher than $50 \mathrm{kHz}$ (Fig. 5.13) [39].

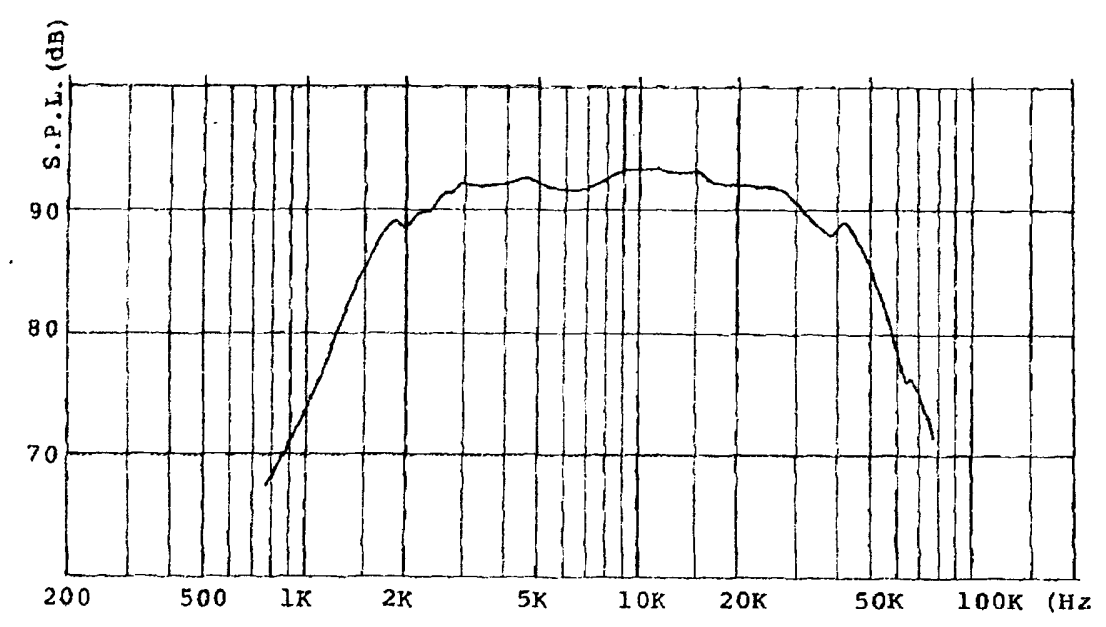

Figure 5.13: The Frequency Response of the novel direct drive ribbon tweeter [39] 
Nieuwendijk [40] in his 1988 paper described in detail the characteristic properties of ribbon tweeters. In this paper, the differences between ribbon loudspeakers and conventional loudspeakers were outlined. Also the paper reported a compact midrange tweeter (Fig. 5.14) [40] driver having an extended frequency range compared with earlier ribbon tweeters. Measurements on the novel midrange tweeter units showed a frequency range of about $800-30000 \mathrm{~Hz}$, low distortion, good sensitivity, and good transient response.

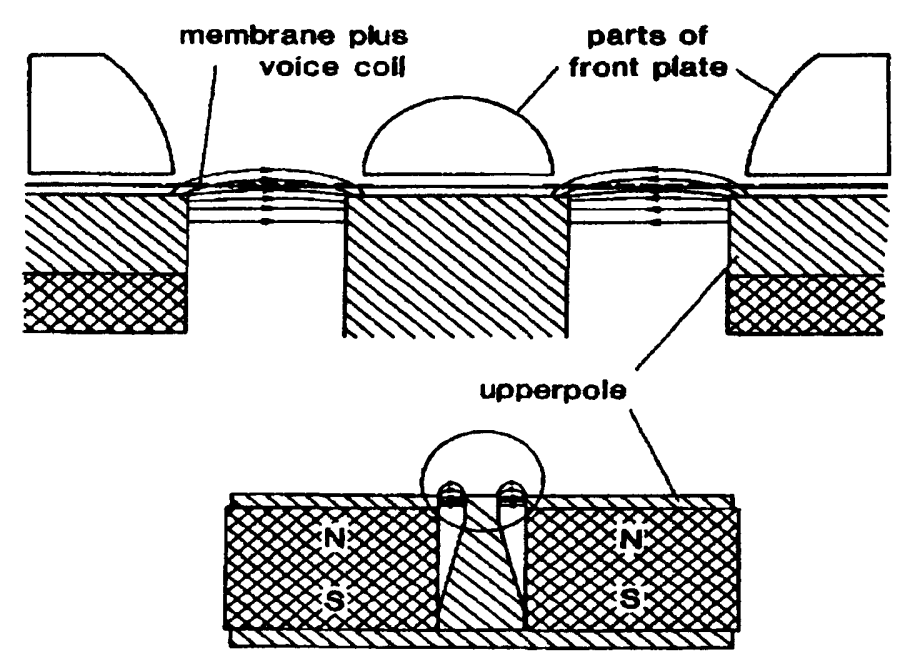

Figure 5.14: Cross-sectional view of the midrange ribbon tweeter [40]

Hayakawa et al. [41] presented improvements in dome loudspeaker characteristics by using a spherical wave-front horn baffle. Conventionally a dome loudspeaker is mounted on a flat baffle such that the sound waves radiating from the diaphragm are subject to reflection, interference, and diffraction, which results in irregular response characteristics. By introducing a horn baffle, the paper claimed that it was possible to spread the wave front without distorting it.

\subsection{Distortion Analysis and Reduction}

In 1944, Harry F. Olson [42] theoretically and experimentally described the effect of a non- linear cone suspension system on loudspeaker performance. The paper showed that most of the unusual phenomena exhibited by the direct radiator loudspeaker at the lower frequencies were due to the non-linear characteristics of the suspension system. One of the effects of a non-linear cones suspension system was a jump phenomenon 
in the response characteristic. Another effect was the production of harmonics and subharmonics due to the non-linear cone suspension system. The distortions due to non-linear suspension system at lower frequencies were significant because of the large amplitude or excursion of the cone at those frequencies. The discussion by Olson was based on an idealized system which had only one resonant frequency, in the case when the displacement was so small that the non-linear effects were negligible. An actual system, of course, has more than one resonant frequency. R.V. L Hartley, in 1944, described in his paper [43] that in multi-resonant non-linear systems, in addition to the harmonic frequencies, inharmonic subfrequencies may also be produced.

W.J. Cunnigham in his 1949 paper [44] discussed the non-linear distortions in loudspeakers due to magnetic effects. The first type of distortion arises because of a force of attraction between the voice coil, carrying a current, and the iron of the field structure. This force varies as the square of the current and produces second harmonic distortion. The force may be related to the space rate of change of self-inductance of the voice coil as it moves in the air gap. The magnitude of the distortion is greater for low frequencies and large currents. Cunningham suggested the distortion may be reduced by proper proportioning of the voice coil and field structure and by using a short-circuited winding on the field structure. The second type of distortion arises due to non-uniformity of the magnetic field in which the voice coil moves. This distortion may be reduced by proportioning the voice coil and field structure so that the mean field in which the coil moves remains as constant as possible.

In 1961, Larson [45] described the effect of transient response on loudspeaker distortion. Larson observed that there was a little correlation between the transient performance of a loudspeaker and musical listening tests. The reason being the psychoacoustic performance of the ear tends to make it insensitive to the shape of the wave envelope of a tone burst and also the echoes in the usual listening room tend to mask the hangover transient of the loudspeaker.

Raichel [46], in 1977, discussed the theoretical minimum levels of harmonic distortion that can be expected in loudspeakers based on the fundamentals of air nonlinearity. The inherent nonlinearity in acoustic propagation generates harmonic 
distortions in loudspeakers. Therefore a minimum amount of harmonic distortion is present even in an ideal loudspeaker. This paper employed a numerical method to derive the distortion curves for an ideal loudspeaker. Real loudspeakers may approach the absolute minima in harmonic distortion, indicated by the distortion curves, but can never excel as claimed by this investigation.

Greiner and Sims [47], in their 1983 paper, described nonlinear distortions and frequency response aberrations in low-frequency loudspeaker systems. A principal nonlinearity in loudspeakers, associated with the magnetic structure, is non-constant $B l$ product versus cone excursion. Another is a voice coil that is not centred, front to back, under zero signal conditions. In addition, nonlinearities in the compliance of the spider and surround are significant. This paper proposed a multiple-loop feedback system to reduce the non-linearity present in low frequency drivers. Experimental results from loudspeaker systems using the proposed feedback system were presented and confirmed that a substantial increase in low-frequency loudspeaker system performance was possible using a very simple hardware implementation.

Richard E. Warner described the effect of negative impedance source on loudspeaker performance [48]. A direct radiator moving coil loudspeaker driven by an amplifier whose output impedance approaches the negative of the blocked voice-coil impedance can be made to exhibit extended low-frequency response with reduced distortion (Fig. 5.15) [48]. The effect of the system is in some ways analogous to a many fold increase in loudspeaker efficiency. In a typical case, neutralization of $70 \%$ of the blocked voice-coil impedance completely damps the cone resonance, as well as substantially reducing the nonlinear distortion below resonance. According to Warner, when the amplifier is compensated for the falling radiation resistance at low frequencies, uniform output can be obtained to any arbitrary low frequency, limited only by the ultimate power-handling capability of the amplifier and speaker. In this system, no additional amplifier power is required at frequencies down to the speaker resonance; additional power is required below that point. 


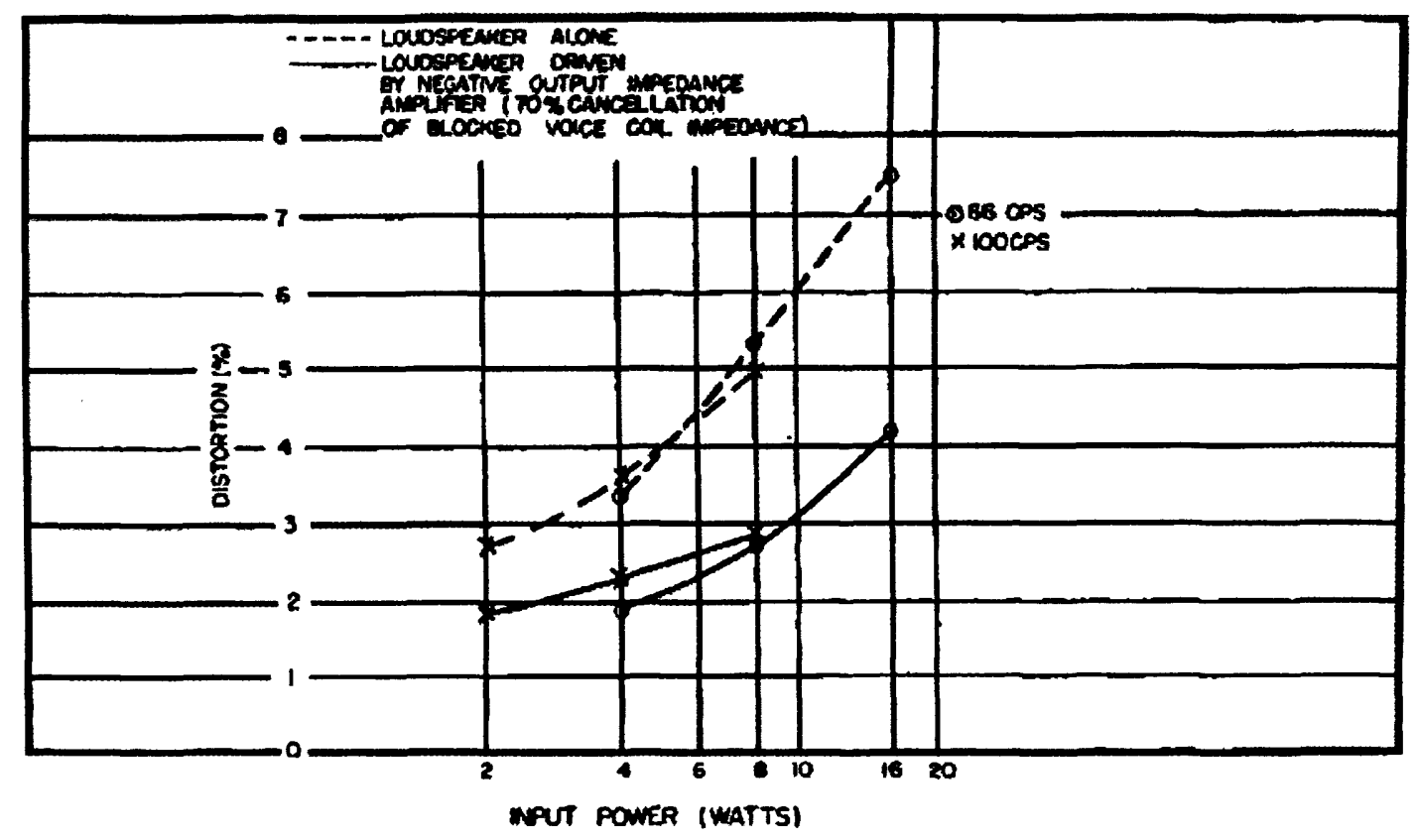

Figure 5.15: The difference in harmonic distortion with and without the negative impedance [48]

Modulation distortion, or the introduction of inharmonic frequencies resulting from mixing two or more input signals, is recognized to exist in amplifiers as amplitudemodulation distortion popularly called intermodulation distortion. In loudspeakers where mechanical motion is also involved, distortion caused by frequency modulation arises due to the Doppler Effect. Beers and Belar [49] were the first to describe frequency modulation distortion in loud speakers in their landmark 1943 paper. Mathematical analysis and measurements presented in this paper indicated the possibility of frequency-modulation distortion in loudspeakers when reproducing a complex sound. Since this distortion increases with frequency (Fig. 5.16) [49], its effects are most pronounced in high-fidelity reproducing systems. Beers and Belar suggested some simple methods for keeping FM distortion products below the level of audibility, such as dividing the spectrum among at least two drivers. In 1982, Allison and Villchur [50] discussed experiments on the magnitude and audibility of frequency modulation (FM) distortion based on the earlier work by Beers and Belar. They also suggested multiple driver system for minimizing Doppler distortion in loudspeakers. 


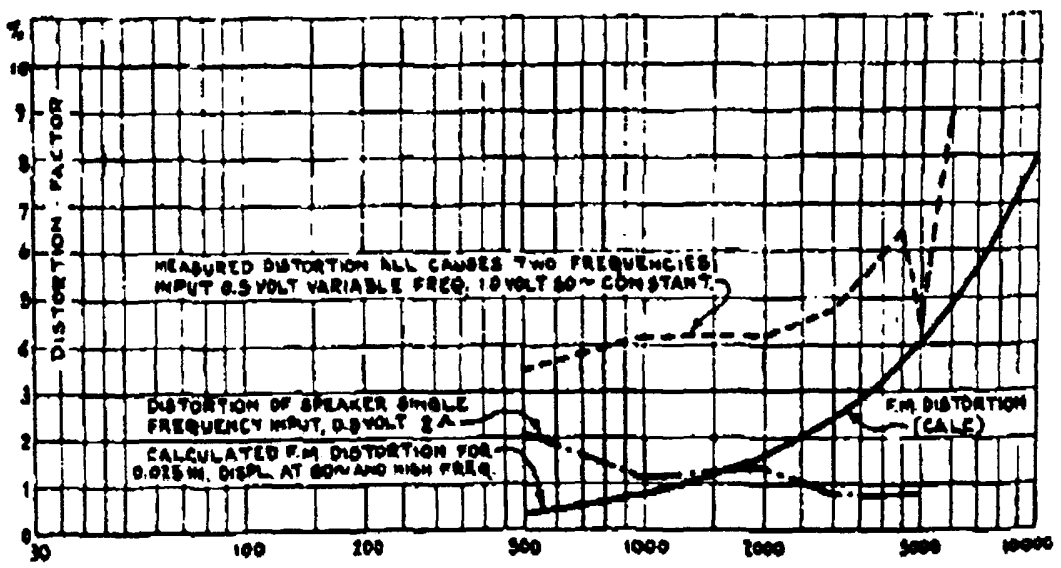

Figure 5.16: Harmonic cross-modulation and frequency modulation distortion of permanent-magnet field loudspeaker [49]

Klipsch [51], in 1968, made a major study of modulation distortion in loudspeakers, basing much of his fervent advocacy of horns on their benefits in its reduction. Modulation distortion in loudspeakers consists of amplitude modulation distortion (AMD)) and frequency modulation distortion (FMD) and the effective sum of these is the total modulation distortion (TMD). Klipsch showed that the first-order side frequencies are due to frequency modulation and the second-order sideband frequencies are due to amplitude modulation. Small direct-radiator loudspeakers typically display large AMD and relatively less FMD, while horn loudspeakers display small FMD and negligible AMD. Klipsch in a later publication [52] suggested that since modulation distortion is usually several times the magnitude of harmonic distortion, and consists of frequencies which are not harmonically related to the input frequencies, modulation distortion is much more important than harmonic distortion. He also mentioned that the horn, vented box, and total enclosure type speakers display increasing amounts of distortion in the given order. Small [53] also discussed crossover networks and modulation distortion. Small concluded that the larger driver will require less diaphragm displacement and will thus produce less modulation distortion.

Digital signal processing has brought about the capability to implement complex mathematical modelling of nonlinearities and the potential to implement correction algorithms in real time. In 1987, Kaizer [54] employed the technique of Volterra series expansion to model the nonlinear response of electrodynamic loudspeakers. 
Using this Volterra series expansion, Kaizer calculated the harmonic and intermodulation distortion products of an actual loudspeaker. The distortion curves predicted by the model and the measured distortion curves showed a reasonable agreement.

Klippel [55] continued the work of Kaizer presented methods for the dynamic measurement and interpretation of the non-linear parameters and large-signal behaviour of electrodynamic loudspeakers at low frequencies, and presented his mirror filter technique for distortion reduction and equalizing woofer response. Klippel continued his work focusing on nonlinear modelling and nonlinear system identification techniques applied to horn loudspeakers [56] and presented methods of compensating for nonlinear distortion of horn loudspeakers by digital signal processing [57].

Y. Nomura and Y. Kajikawa [58] proposed a linearization (compensation of nonlinear distortion) method for loudspeaker systems using the MINT (Multiple-input/output Inverse-filtering Theorem) and Volterra filters. Experimental results demonstrated that the proposed method has about $20 \mathrm{~dB}$ higher performance than the conventional one. In 2006, K. Lashkari [59] introduced a novel method for distortion reduction. When small loudspeakers are driven at high playback levels the nonlinear characteristics of these speakers become a major source of sound degradation Conventional approaches to loudspeaker compensation based on the Volterra model improve the sound quality only at low playback levels and may introduce more distortion at high playback levels. Lashkari claimed that a new Volterra-Wiener model is a better match to the loudspeaker response and lends itself to having an exact nonlinear inverse. Experimental results showed that the compensation based on the new model greatly reduced the linear and nonlinear distortions of small loudspeakers.

\subsection{Measurement}

Loudspeaker performance measurement technique has been the subject of investigation since the quality of reproduced sound became an issue for the consumers. Different researchers at various times have proposed several measurement techniques in order to quantify the quality of the reproduced sound. The following 
gives a brief description of the various methods used by the researchers for loudspeaker measurement.

In 1962, Villchur [60] discussed the two most popular measurement methods at that time. The white noise technique was able to reveal even subtle distortions in the texture of reproduced sound related to ringing; uneven presentation of acoustical energy in different parts of the frequency spectrum, dispersion, etc. The live versus recorded technique (Fig. 5.17) [60] established a reference standard, providing validation of the test technique. The two techniques together made up what had proven to be very sensitive and reliable test for speaker evaluation.

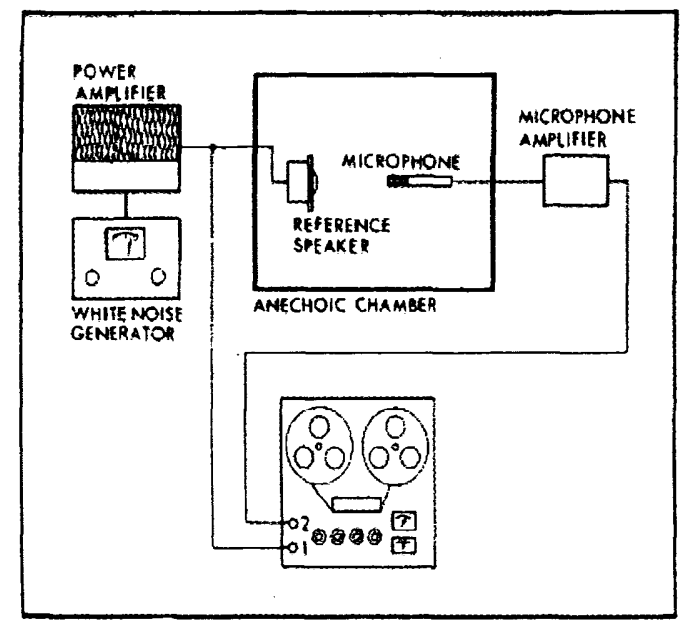

Figure 5.17: Test setup for live versus recorded technique [60]

Sioles [61] described loudspeaker measurements in live rooms in 1963. The paper described room acoustics theory and presented data (Fig. 5.18) [61] depicting the reverberant field response of a typical directional and a nondirectional loudspeaker. The measurements were taken with sinusoidal input as well as $1 / 3$ octave bands of random noise. Additionally, in this paper, the effect of source position relative to the room boundaries was studied in sufficient detail to draw some rather interesting conclusions. 


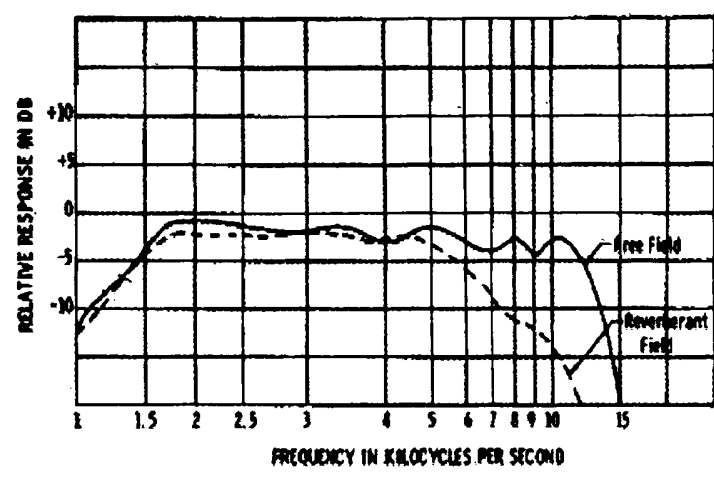

(a)

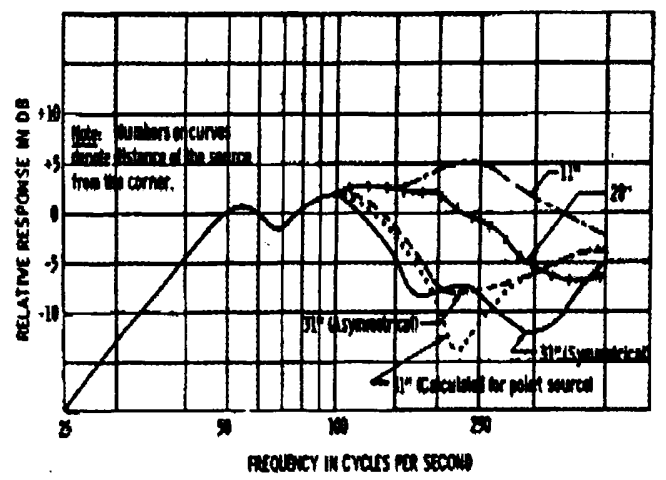

(b)

Figure 5.18: (a) High Frequency Responses of two different fields (b) Low frequency response in various corner positions [61]

V. Kaminsky [62] discussed the response of loudspeakers to tone bursts (Fig. 5.19) [62], the standard technique at the time for evaluating loudspeaker transient response. Studies were conducted on loudspeaker parameters including transient response, damping, resonances and spurious radiation in direct and horn-type radiators under conditions of infinite baffle (anechoic) and other enclosure modes. Resonance modes in horns exclusive of driver resonances were also studied in this paper.
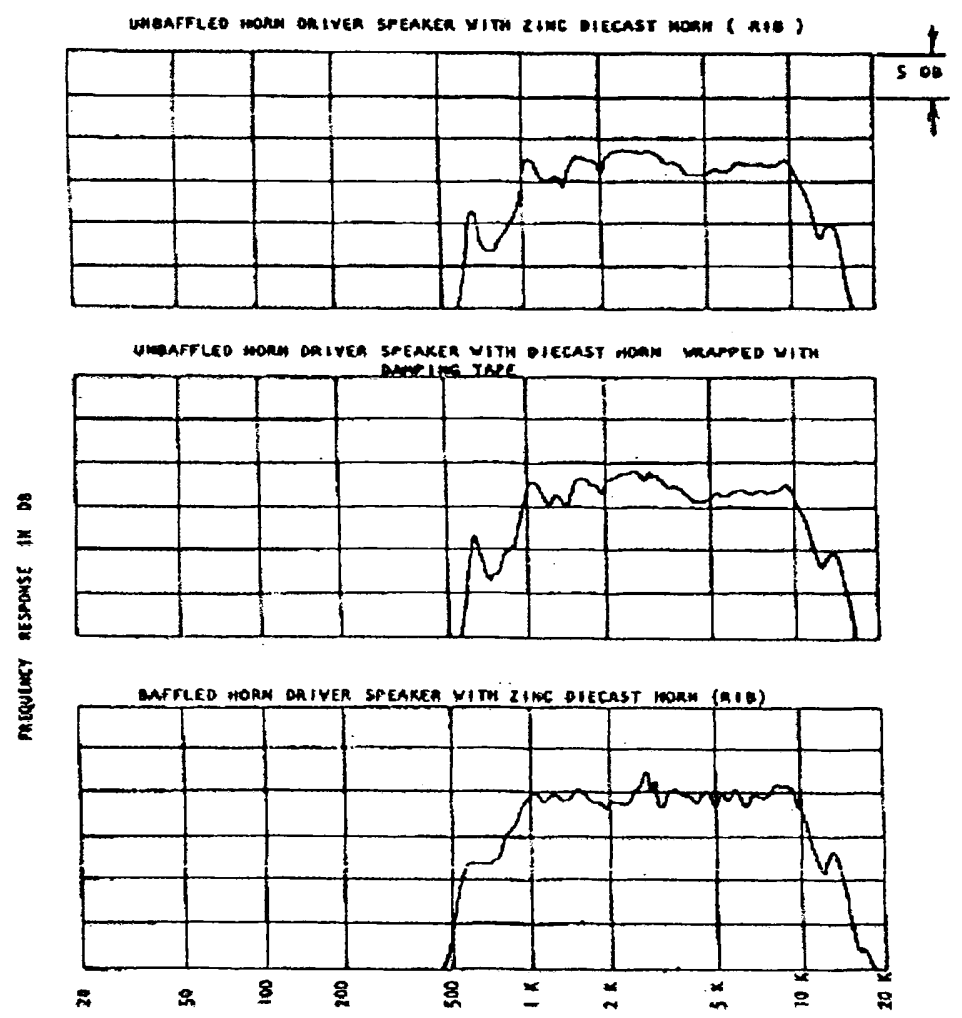

Figure: 5.19: Horn driver frequency response curves using tone burst technique [62] 
The application of rigorous mathematical analyses and the availability of digital measurement techniques brought about a real revolution in the methods and interpretation of loudspeaker measurements. Heyser [63] (Time Delay Spectrometry) and Berman and Fincham [64] (Digital Processor) and Fincham [65] (Improvement of digital processing) were the pioneers in applying these new tools and methods. It is interesting to compare the digital displays of impulse responses and the cumulative decay spectra with the tone-burst transient testing perspective of two decades earlier. Bunton and Small [66] discussed this in detail, comparing and contrasting cumulative spectral analysis with traditional tone-burst testing, and recommending mathematical methods for generating more easily interpreted spectral displays.

Nomoto et al. [67] presented techniques for observing three-dimensional loudspeaker wavefront propagation and transient response. This paper described a method in which a microphone scanned a given plane within a sound field, data from which measurements were recorded by a computer on magnetic tape. When this magnetic tape was run again, the patterns of sound pressure distribution at different instants of time were displayed on a cathode ray tube in perspective and filmed using a motion picture camera. When this film was projected at the correct speed, it was possible to understand how the sound field extends in space and changes in structure with time.

The earliest uses of lasers in loudspeaker measurement were reported by Hladky in 1974 [68] and Fryer in 1975 [69]. At this time the technique, known as holographic interferometry (Fig. 5.20) [69] was the only option. The method produced a contourtype display of variations in vibration amplitude across the test surface, using intensity changes ('light' to 'dark') to signify the amplitude changes. Frankort used an early 'time-averaged' holographic system to compare the behaviour of practical cone loudspeakers with his theoretical analysis in a fascinating study [70]. The earlier systems suffered from difficulties with sensitivity and with real-time or 'live' information, although Fryer described techniques to partially overcome these problems. Real-time methods were then feasible with the use of video systems (replacing the earlier photographic-plate image capture) as detailed by Tyrer [71], giving rise to a technique called electronic speckle pattern interferometry (ESPI). 


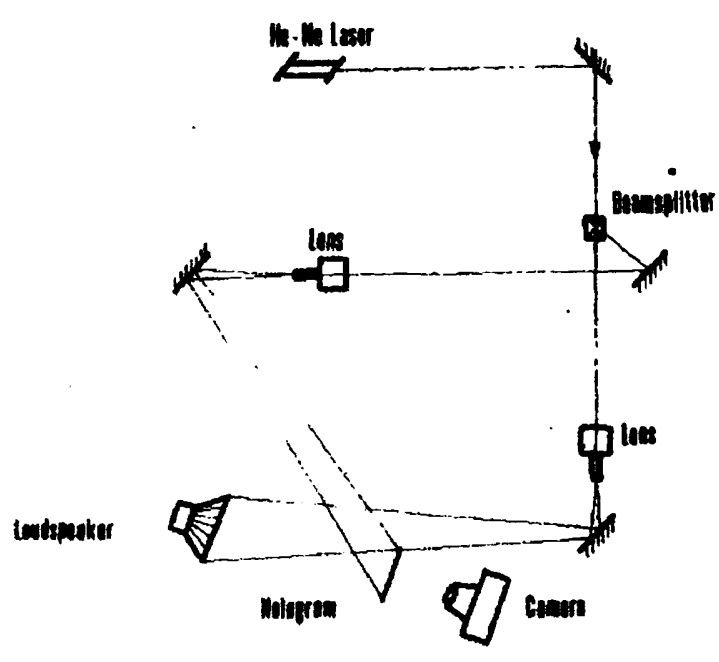

(a)

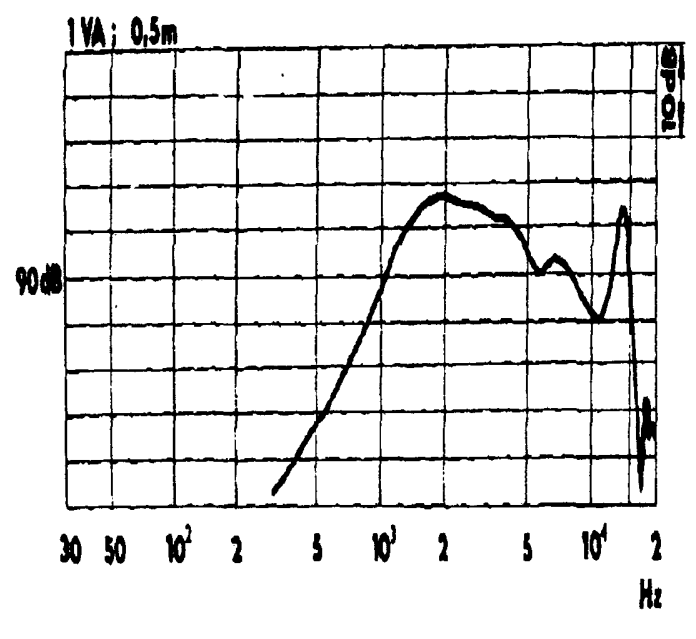

(b)

Figure 5.20: (a) Measurement setup using holography (b) Frequency response of a dome tweeter using holography technique [69]

The major disadvantage of holographic interferometry was the difficulty in obtaining phase information. The development of Doppler interferometry provided the solution to this problem. With this technique a laser beam incident upon a moving surface was modulated with a carrier frequency (e.g. $40 \mathrm{MHz}$ ). The light reflected by the moving object would be 'Doppler' frequency-shifted, and the frequency of the returned light was an encoded measurement of the local velocity amplitude of the surface. The key feature was that the carrier frequency could be increased or decreased according to the direction of movement of the surface. Hence velocity phase information was also present in the reflected light beam. The use of such a system was reported in 1980 by Bank \& Hathaway [72]. In their investigation the beam from a laser vibration interferometer was optically raster scanned over a vibrating surface and a phasesensitive detector provided velocity information at any phase of the motion. These data were then digitally processed and a hard copy print gave a three-dimensional isometric view of the complete vibrating surface of the test object frozen (Fig, 5.21) [72]. The system developed by Bank \& Hathaway was modified in 1981 to provide an animated display of the vibration of loudspeaker diaphragms. 


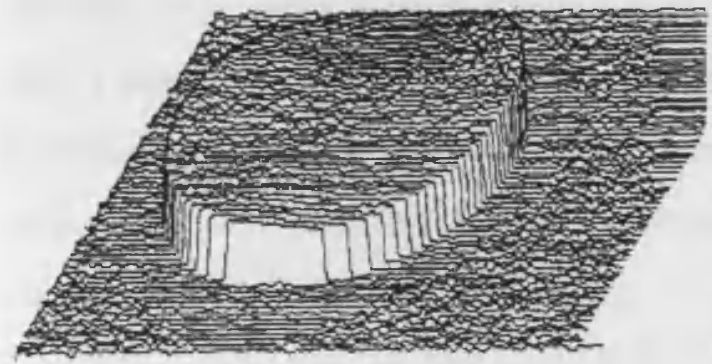

(a)

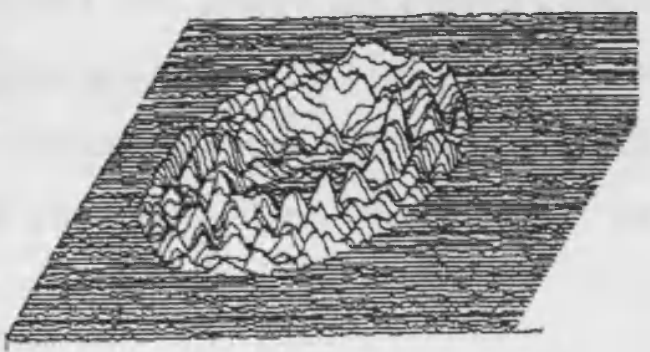

(b)

Figure 5.21: Three dimensional vibrational surface of a cone driver at (a) $95 \mathrm{~Hz}$ and (b) $5 \mathrm{kHz}$ [72]

With the improvement of methods for Finite Element Analysis, an attempt to correlate simulation and measurement was reported by Henwood et al. in 1987 [73], and early results seemed promising. The use of lasers in the analysis of loudspeaker diaphragms was firmly established in the late 80 's. However, the behaviour of loudspeaker cabinets had not been reported in detail. Early laser systems were too insensitive to measure vibrations of cabinet panels, which had velocity amplitudes typically of the order of 20-30dB less than loudspeaker diaphragms. In addition, the increased size of the object required larger scan areas. This led to either a) greater demand on scanning systems or b) increased distance between test object and optical system. In effect, the major problem with cabinet measurement was that of poor signal-to-noise ratios.

J.R. Wright [74] in 1990 published a breakthrough paper on a new and automated structural analysis system utilising laser Doppler interferometry for non-contact vibration measurement. The new measurement system for non-contact vibration analysis had been developed, using the scanning laser interferometry method to measure local velocities of a vibrating surface. The greatest advantage of this new technology was that, in addition to displacement magnitude, it could also provide phase information which was of vital importance to acoustic and vibration engineers. The paper also investigated the effect of cabinets on loudspeaker panel movement.

Weaver and Leach [75] described an optical technique for measurement of the peakto-peak large signal displacement of a loudspeaker driver cone under dynamic operating conditions. The method also allowed the identification and measurement of shifts in the cone zero centre position as functions of drive level and frequency. 
Moreno [76] used a laser velocity transducer and two-channel fast Fourier transform (FFT) analysis to determine loudspeaker parameters (Fig. 5.22) [76]. In this method, random noise was used to excite the loudspeaker and three important loudspeaker parameters were measured. The measured parameters were input voltage, input current, and diaphragm velocity.

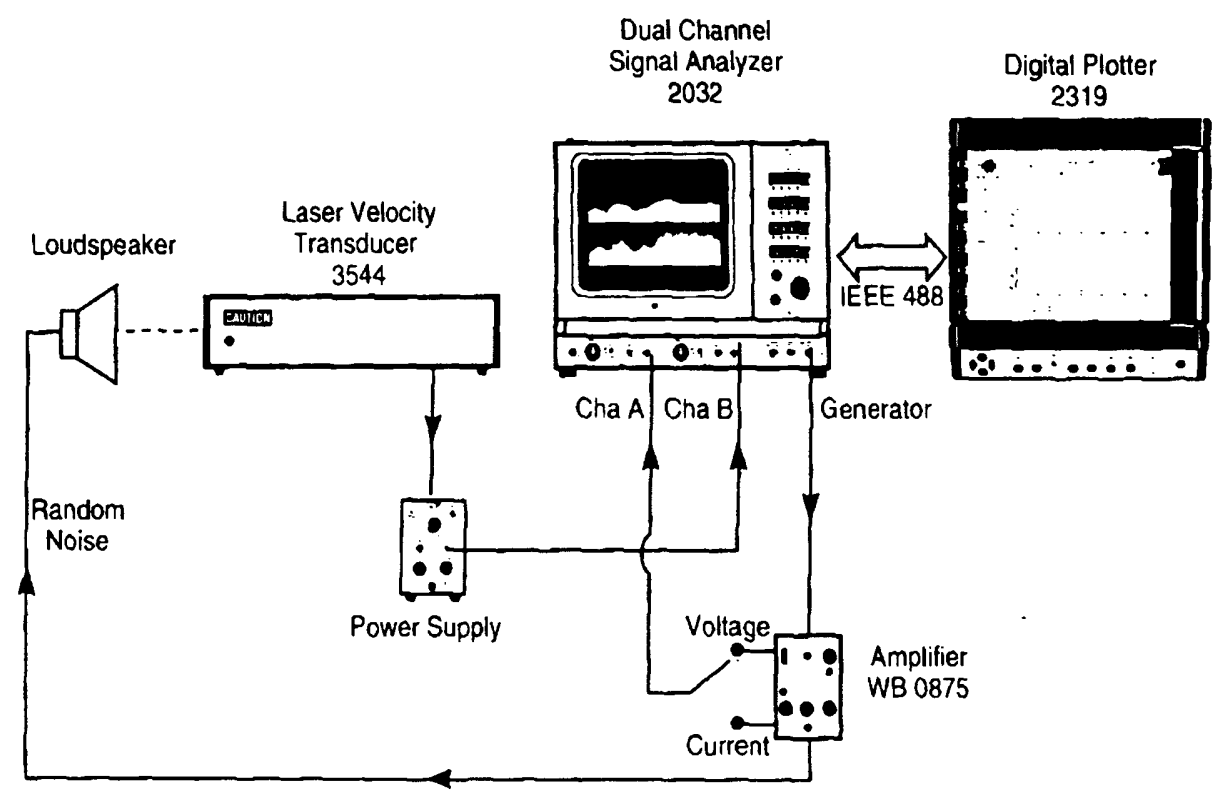

Figure 5.22: Block diagram of measuring setup using laser velocity transducer and fast Fourier transform (FFT) analysis [76]

\subsection{Flat Panel Loudspeaker}

Limited information on the Distributed Mode Loudspeaker (DML) first appeared in the publication of the International Patent Application in April 1997 (Patent No. W097/09842). At its core this Patent application described an acoustic panel of superior bandwidth and radiation properties. N. Harris and M. Hawksford [77] published a landmark paper in 1997 in which they presented the idea of a DistributedMode Loudspeaker (DML) that could work as a Broad-Band Acoustic Radiator. The paper described the principles of a new class of broad-band frequency independent broad-band frequency independent acoustic radiator (DML). The groundbreaking research demonstrated that a low-loss panel with optimal modal distribution produced a flat power response. The paper showed, using numerical method, the bandwidth of a 
DML depends only on the ratio of magnet mass to coil mass. Therefore, the paper claimed, it was possible to design a single DML to be substantially flat in pressure and power response over a very wide bandwidth without any electrical filters. The theoretical results presented in this paper for a flat panel speaker indicated the arrival of a new technology which can cover the entire audible frequency without the need for a cross-over network, something which is vital for broad-band sound reproduction by a traditional piston loudspeaker.

In 1997, M. Colloms and C. Ellis published [78] a paper that examined the application of DML in Home Theatres. The aim of this paper was to examine the theory and practical performance of this new speaker technology in the context of multimedia Home Theatre. The application of DML in home theatre was proposed due to the flat panel characteristics of having wide directivity, constant sound power with frequency and diffuses acoustic output. In this paper the authors outlined the basic theory of this diffuse sound radiator and then applied it to acoustic models developed to compare the sound distribution of established cone type pistonic speakers with the distributed mode type in the context of multi channel sound systems. The preliminary test results in this investigation showed improvement for the Distributed Mode Loudspeaker in the Home Theatre application in respect of sound field generation, centre channel duty and an ability to significantly improve the sound level balance over the audience region for multiple audio channels.

M. Colloms and P. Mapp [79] in another publication carried out a series of investigations to examine the theoretical considerations and the subjective view expressed by a number of observers, that the distributed mode panel loudspeakers appeared to have better speech intelligibility than conventional cone devices. Tests were carried out with the devices operating both in isolation and in combination and compared to traditional cone transducers. A number of parameters were investigated and comparisons made between the technologies under different listening environments. The measurements and subjective tests reported in this paper supported the anecdotal evidence that Distributed Mode loudspeakers can offer significant objective and subjective advantages over conventional loudspeaker technology. 


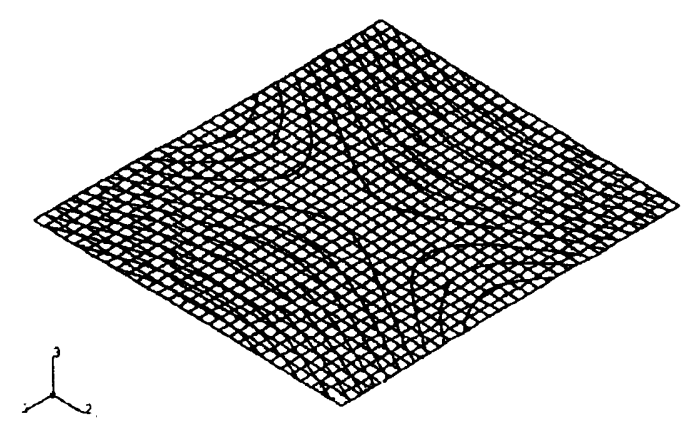

(a)

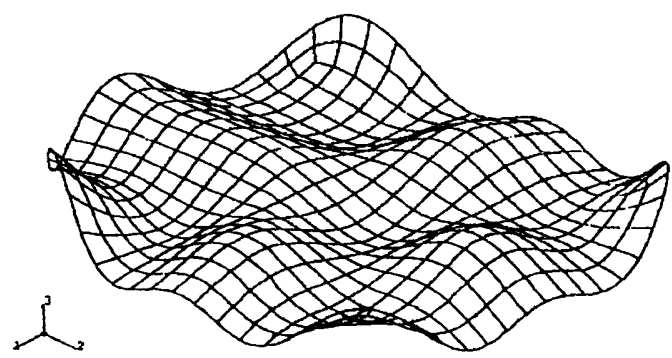

(b)

Figure 5.23 : Panel Acoustic Modes - (a) Non Diffuse (b) Diffuse [80]

Henry Azima and Neil Harris [80] examined the acoustic response of a diffuse radiator (Fig. 5.23) [80] in the vicinity of simple boundaries using finite element analysis and analytical methods. Traditional phase-coherent acoustic radiators are subjected to destructive interference when they interact with their boundaries. The paper discussed the flat panel speakers whose radiation is spatially and temporally diffuse, mitigating the problem of interference by producing 'sympathetic' boundary reflections. Results from computer simulations for both classes of radiator were presented and these were compared to single boundary and listening room measurements. The results of the finite element analysis (FEA) simulation showed that the acoustic radiation from a point source and a diffuse radiator line source is broadly similar in free-space but this is not true when boundaries were present. In contrast to a distributed mode loudspeaker (DML), whose radiation is not greatly changed by the presence of boundaries (Fig. 5.24) [80], the radiation from a piston loudspeaker is particularly affected in the mid-band frequencies. This investigation, due to the limitations of the conventional measurement and assessment techniques, could not support conclusively the simulation findings with measurement results.

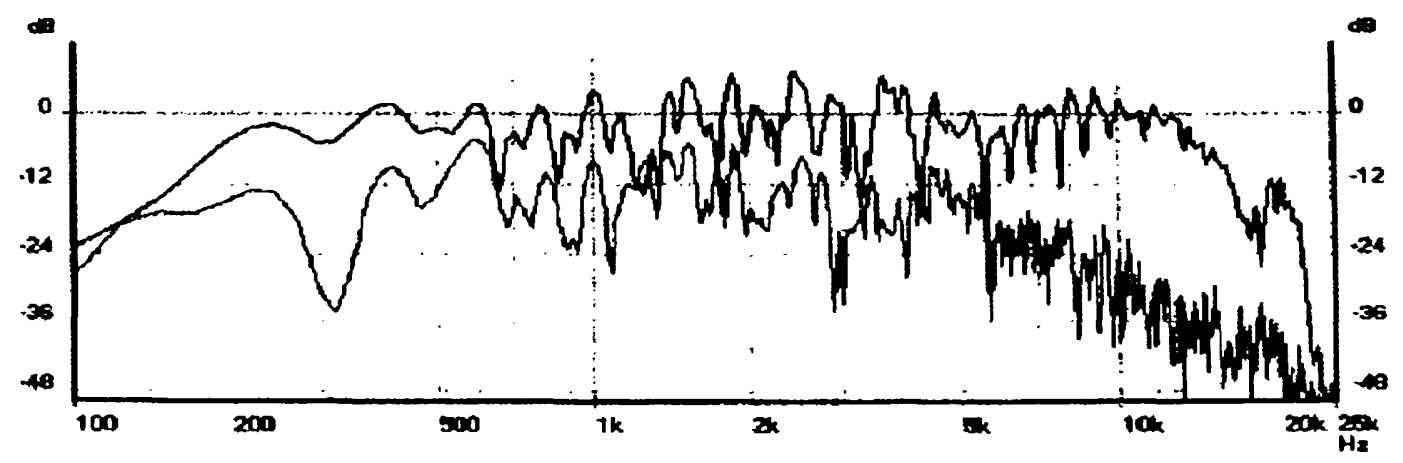

Figure 5.24: Reflected (lower trace) vs. free-field response of DML [80] 
Another interesting comparative study between the distributed mode loudspeaker and the conventional ones had been presented by Graham Bank and Neil Harris [81]. The paper presented a theoretical model of the distributed mode loudspeaker (DML) and compared it to that of a conventional, mass-controlled loudspeaker. Electromechanical modelling results were compared to real measurements of example DMLs. The implications of uniform directivity and diffuse radiation to room interactions were also discussed. The study concluded that the DML have a number of advantages over conventional loudspeakers. Graham Bank [82] in a later publication proposed that, because of the diffuse nature of the radiation from a DML panel, such an acoustic radiator should be scalable. The results presented in this paper showed that the polar characteristics for panels of two different sizes were substantially the same and confirmed that the directional characteristics of a DML panel were the same, irrespective of size.

The exciter component in a distributed mode loudspeaker (DML) can have a profound effect on the overall performance of the system. The paper by Martin Robert [83] in 1997 introduced the equivalent circuit analysis of the combination of electro-dynamic exciter and distributed mode panel and discusses how exciter parameters can influence DML performance. Measurements are given which show how these influences are manifested. Two different constructions of the DML exciter were discussed. One was the 'Bender' type and other was the 'Inertial' type (Fig. 5.25) [83].

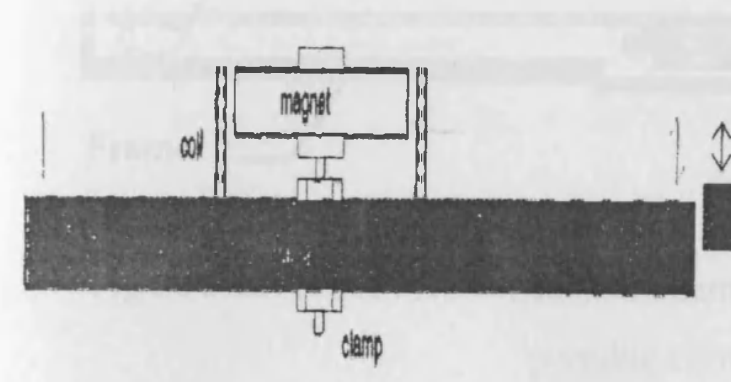

(a)

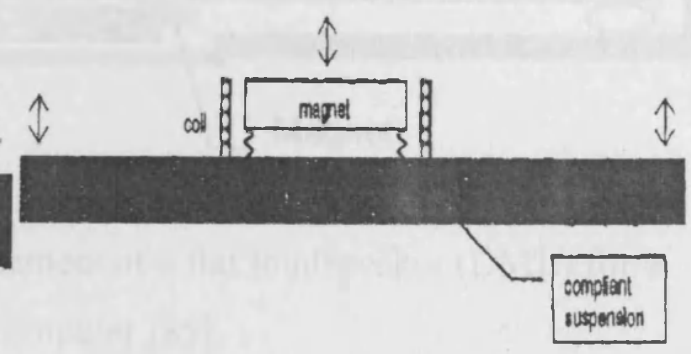

(b)

Figure 5.25: (a) Bender Type electro-dynamic exciter (b) Inertial Type electrodynamic exciter [83] 
Distortion mechanisms in flat panel speakers have been explored by M. Colloms et al. [84] in a paper in 1998. Acoustic radiation from a Distributed Mode Loudspeaker (DML) results from low amplitude bending waves. Compared with the motor system of a pistonic driver, the DML exciter is of subtly different design and equivalent circuit with a different relationship to the radiating diaphragm. In this paper, loudspeaker distortions were reviewed, the equivalent circuits modelled and compared with the DML case, and the results for comparative measurements were presented. The investigation in this paper concluded that the distortion from DM panel speakers is significantly lower than equivalent piston/cone speakers, typically by $10 \mathrm{~dB}$.

Tashiro et al. [85] in 1997 presented the idea of integrating a flat-panel distributedmode loudspeaker into a notebook computer (Fig. 5.26) [85]. The most basic form of a practical Distributed Mode Loudspeaker consists of a panel driven at one point (or a near approximation to one point) by an electro-dynamic inertial type exciter. Such an exciter consists of a voice coil rigidly bonded to the panel which in turn sits in the magnetic gap of a conventional loudspeaker type magnet assembly resiliently mounted on the panel. The flat panel technology, when incorporated into a laptop computer, exhibited flatter frequency response compared to that of a traditional loudspeaker.

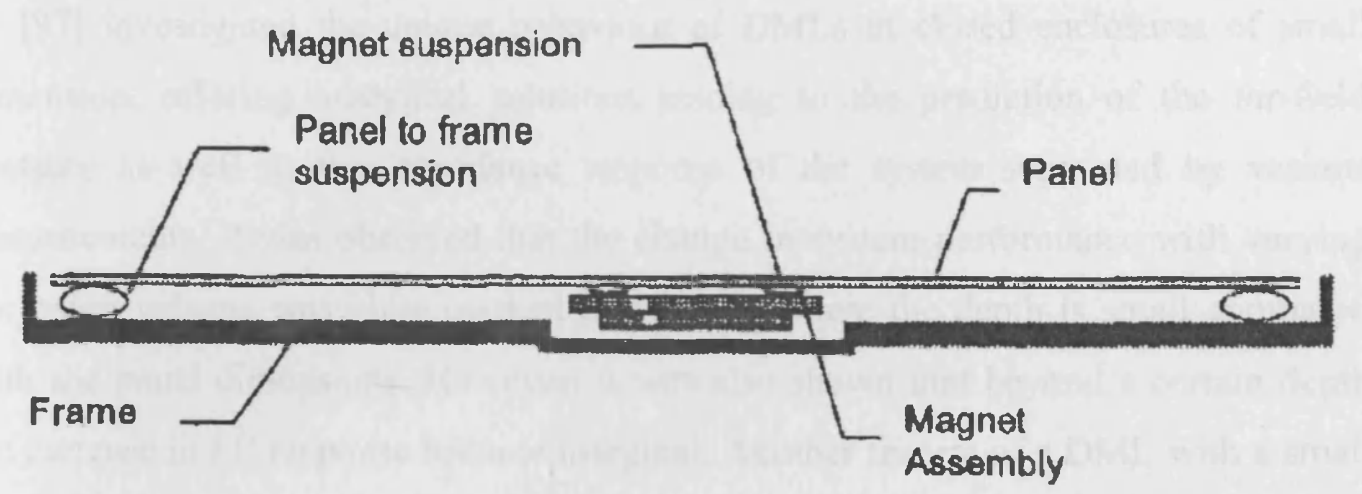

Figure 5.26: Basic mechanical arrangement of a flat loudspeaker (DML) for a portable computer [85]

M. Antila et al. [86] reported a flat-panel electromechanical film (EMF) loudspeaker (Fig. 5.27) [86] that operated on an electrostatic principle. In the EMF loudspeakers, the electromechanical film vibrated inside the porous plates in the electric field 
producing sound. The size of the loudspeaker panel was $500 \mathrm{~mm}$ x $600 \mathrm{~mm}$. Nine panels were joined together with a $3 \times 3$ configuration to form a large, flat surface and each panel was driven with an individual signal. Practical tests on this speaker showed that an additional sub-woofer was mandatory in order to satisfactorily produce the full audio spectrum.

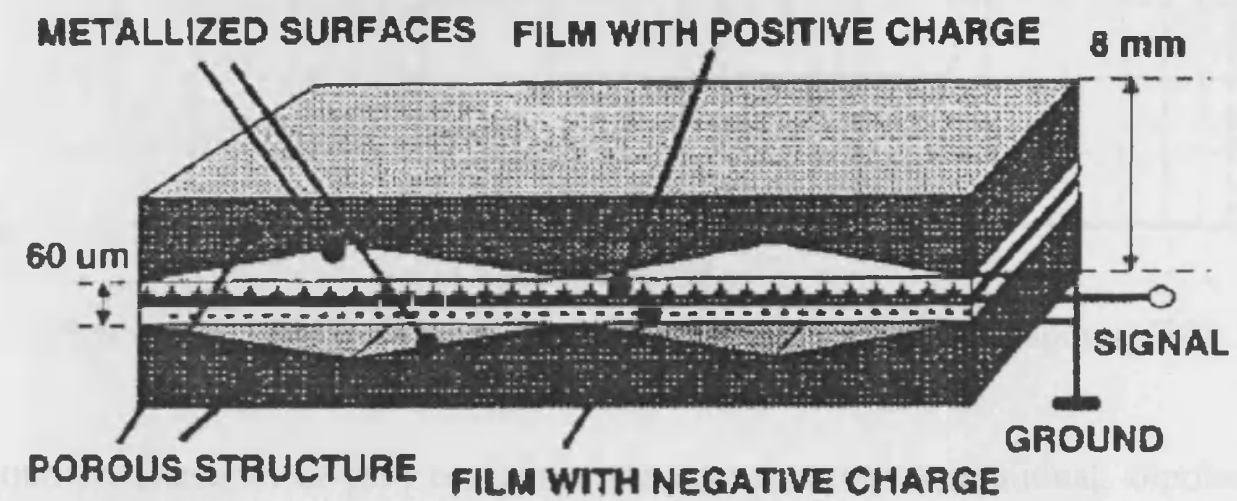

Figure 5.27: The cross-section of the EMFi panel loudspeaker [86]

As in the case of a conventional loudspeaker enclosure, the rear radiation may be a hindrance to the best performance of the DML system. The paper by Henry Azima et al. [87] investigated the unique behaviour of DMLs in closed enclosures of small dimension, offering analytical solutions leading to the prediction of the far-field pressure as well as the impedance response of the system supported by various measurements. It was observed that the change in system performance with varying enclosure volume was quite marked in the case where the depth is small compared with the panel dimensions. However, it was also shown that beyond a certain depth the increase in LF response become marginal. Another feature of a DML with a small enclosure was seen to be a significant improvement in the mid and high frequency response of the system.

In 2000, James Angus [88] showed that a flat-frequency response in a DML is possible but only under certain conditions (Fig. 5.28) [88]. The paper showed that a combination of wave propagation mechanisms was responsible for DMLs broad frequency response. To achieve a flat frequency response was a challenge and 
required careful design of a composite material overall bending modulus, core shear modulus, faceplate bending modulus, and thickness of the whole assembly.

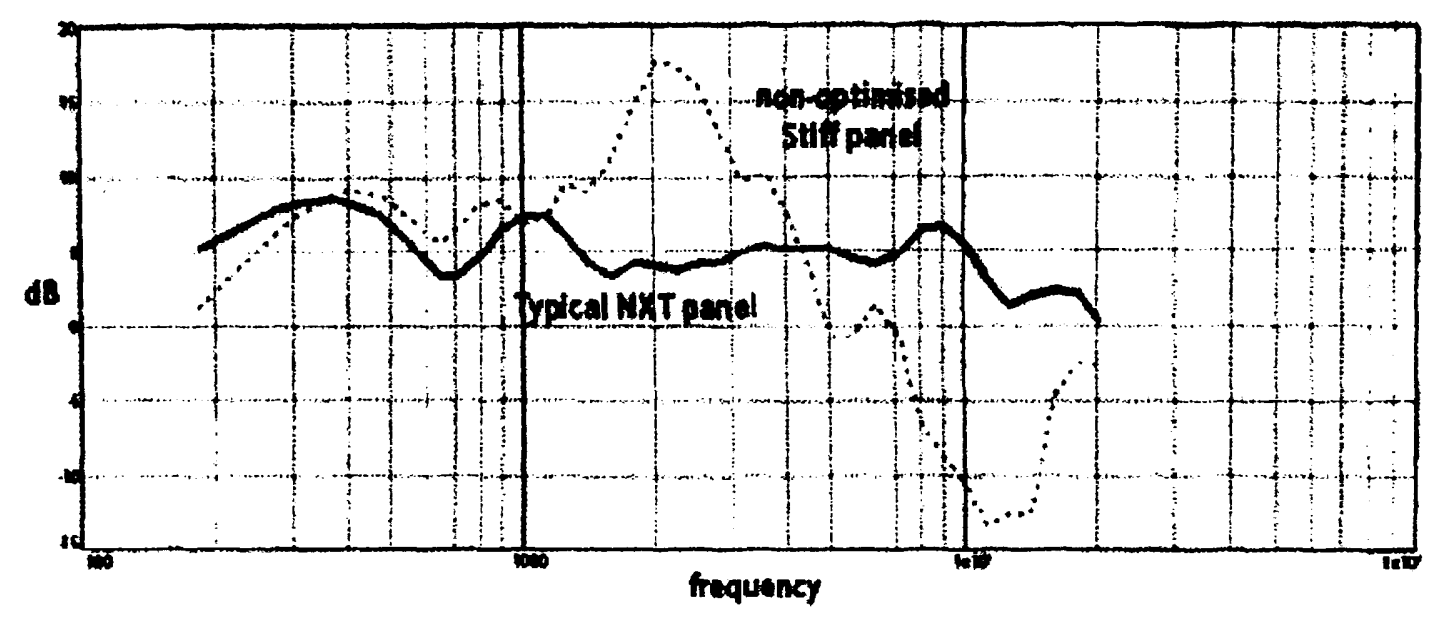

Figure 5.28: NXT's published typical flat panel frequency response [88]

In $2000 \mathrm{~N}$. Harris et al [89] compared the acoustics of conventional, dipolar, and panel loudspeakers in the presence of a dominant specular reflection, using both measurement and numerical simulation. The degree of correlation between the direct and indirect sounds was investigated. The study confirmed that the DML sources produce reflections that are less correlated to the direct sound than those radiated from piston sources.

Transducers are used in arrays for a wide variety of purposes. In reproduced sound, loudspeaker arrays are used because they allow some control over the directivity and frequency response of the radiated sound. However, the performance of the array can be limited by the response of the individual transducer elements. It is difficult to form an omnidirectional array response if the individual transducers are highly directional, as happens with pistonic sources at high frequency. In 2002, Lee D Copley et al. [90] explored the usefulness of Distributed Mode Loudspeakers (DML) in the formation of omnidirectional array responses. This paper concluded that by using DML array elements, the directivity of the array was more constant and broad over a wide frequency bandwidth than would be obtained with pistonic sources. However, the response in the low-frequency sparse modal region was far from omnidirectional. 


\section{References:}

1. C. W. Rice, E. W. Kellogg, "Notes on the Development of a New Type of Hornless Loudspeaker," J. Audio Engineering Society, vol. 30, Issue 7/8, pp. 512-521 (reprint 1982).

2. H. F. Olson, "Multiple Coil, Multiple Cone Loudspeaker," J. Acoustical Society of America, Vol. 10, Issue 3, pp. 256-257 (1939)

3. H. F. Olson, J. Preston, E. G. May, "Recent Developments in Direct-Radiator High-Fidelity Loudspeakers," J. Audio Engineering Society, Vol. 2, Issue 4, pp. 219-227 (1954)

4. A. B. Cohen, "The Mechanics of Good Loudspeaker Design," J. Audio Engineering Society, Vol. 2, Issue 3, pp. 176-182 (1954)

5. W. T. Fiala, "Recent Cone Speaker Developments," J. Audio Engineering Society, Vol. 7, Issue 3, pp. 110-114 (1959)

6. R. J. Parker, "Permanent Magnets in Audio Devices," IRE Transactions on Component Parts, Vol. 5, Issue 1, pp. 32-37 (1958)

7. R. J. Parker, "Trends in Loudspeaker Magnet Structure," J. Audio Engineering Society, Vol. 12, Issue 3, pp. 236-239 (1964)

8. J. King, "Loudspeaker Voice Coils," J. Audio Engineering Society, Preprint No. $634,36^{\text {th }}$ Convention (1969)

9. J. R. Gilliom, P. L. Boliver, L. C. Boliver, "Design Problems of High-Level Cone Loudspeakers," J. Audio Engineering Society, Vol. 25, Issue 5, pp. 294299 (1977) 
10. W. Bottenberg, L. Melillo, K. Raj, "The Dependence of Loudspeaker Design Parameters on the Properties of Magnetic Fluids," J. Audio Engineering Society, Vol. 28, Issue 1/2, pp. 17-25 (1980)

11. R. J. Newman, P. F. Fidlin, "New Magnetic System Designs for SoundReinforcement Loudspeaker Applications," J. Audio Engineering Society, Vol. 37, Issue 4, pp. 215-225 (1989)

12. D. J. Button, M. R. Gander, "The Dual Coil Drive Loudspeaker," AES UK Conf. Microphones \& Loudspeakers, The Ins \& Outs of Audio (MAL), Paper No. MAL-14 (1998)

13. J. R. Wright, "An Empirical Model for Loudspeaker Motor Impedance," J. Audio Engineering Society, Vol. 38, Issue 10, pp. 749-754 (1990)

14. R. Beljan, J. R. Wright, "Comments on -An Empirical Model for Loudspeaker Motor Impedance- and Author's Reply,” J. Audio Engineering Society, Vol. 40, Issue 1/2, pp. 42-43 (1992)

15. C. Zuccatti, "Optimizing the Voice-Coil-Airgap Geometry for Maximum Loudspeaker Motor Strength," J. Audio Engineering Society, Vol. 45, Issue 5, pp. 357-359 (1997)

16. J. Vanderkooy, P. M. Boers, R. M. Aarts, "Direct-Radiator Loudspeaker Systems with High Bl," J. Audio Engineering Society, Vol. 51, Issue 7/8, pp. 625-634 (2003)

17. R. M. Aarts, "High-Efficiency Low-Bl Loudspeakers," J. Audio Engineering Society, Vol. 53, Issue 7/8, pp. 579-592 (2005)

18. M. R. Gander, "Dynamic Linearity and Power Compression in Moving-Coil Loudspeakers," J. Audio Engineering Society, Vol. 34, Issue 9, pp. 627-646 (1986) 
19. C. A. Henricksen, "Heat-Transfer Mechanisms in Loudspeakers: Analysis, Measurement, and Design," J. Audio Engineering Society, Vol. 35, Issue 10, pp. 778-791 (1987)

20. D. J. Button, "Heat Dissipation and Power Compression in Loudspeakers," J. Audio Engineering Society, Preprint No. $2981,89^{\text {th }}$ Convention (1990)

21. D. A. Barlow, "The Development of a Sandwich-Construction Loudspeaker System,” J. Audio Engineering Society, Vol. 18, Issue 3, pp. 269-281 (1970)

22. F. J. M. Frankort, "Vibration Patterns and Radiation Behaviour of Loudspeaker Cones," J. Audio Engineering Society, Vol. 26, Issue 9, pp. 609$622(1978)$

23. K. Suzuki, I. Nomoto, "Computerized Analysis and Observation of the Vibration Modes of a Loudspeaker Cone," J. Audio Engineering Society, Vol. 30, Issue 3, pp. 98-106 (1982)

24. A. J. M. Kaizer, A. Leeuwestein, "Calculation of the Sound Radiation of a Non-rigid Loudspeaker Diaphragm Using the Finite-Element Method," J. Audio Engineering Society, Vol. 36, Issue 7/8, pp. 539-551 (1988)

25. C. J. Struck, "Investigation of the Non-rigid Behaviour of a Loudspeaker Diaphragm Using Modal Analysis," J. Audio Engineering Society, Vol. 38, Issue 9, pp. 667-675 (1990)

26. T. Shindo, O. Yashima, H. Suzuki, "Effect of Voice-Coil and Surround on Vibration and Sound Pressure Response of Loudspeaker Cones," J. Audio Engineering Society, Vol. 28, Issue 7/8, pp. 490-499 (1980)

27. A. Dobrucki, "Graphic Method of Investigation of the Surround and VoiceCoil Influence on Vibration of Loudspeaker Cones," J. Audio Engineering Society, Vol. 31, Issue 6, pp. 448-450 (1983) 
28. I. C. Shepherd, R. J. Alfredson, "An Improved Computer Model of DirectRadiator Loudspeakers," J. Audio Engineering Society, Vol. 33, Issue 5, pp. 322-329 (1985)

29. H. Suzuki, J. Tichy, "Radiation and Diffraction Effects of Convex and Concave Domes," J. Audio Engineering Society, Vol. 29, Issue 12, pp. 873881 (1981)

30. D.A. Barlow, G.D. Galletly, J. Mistry, "The Resonances of Loudspeaker Diaphragms," J. Audio Engineering Society, Vol. 29, Issue 10, pp. 699-704 (1981)

31. K. Ishiwatari, N. Sakamoto, H. Kawabata, H. Takeuchi, T. Shimizu, “ The Use of Boron for High-Frequency Dome Loudspeakers," J. Audio Engineering Society, Vol. 26, Issue 4, pp. 217-220 (1978)

32. N. Sakamoto, K. Satoh, K. Satoh, N. Atoji, " Loudspeaker with Honeycomb Disk Diaphragm," J. Audio Engineering Society, Vol. 29, Issue 10, pp. 711 $719(1981)$

33. H. Niguchi, O. Yamamoto, M. leki, T. Shimizu, N. Sakamoto, "Reinforced Olefin Polymer Diaphragm for Loudspeakers," J. Audio Engineering Society, Vol. 29, Issue 11, pp. 808-813 (1981)

34. T. Yamamoto, T. Tsukagoshi, T. Tohma, M. Kimura, F. Tanaka, "HighFidelity Loudspeakers with Boronized Titanium Diaphragms," J. Audio Engineering Society, Vol. 28, Issue 12, pp. 868-873 (1980)

35. T. Tsukagoshi, S. Yokozeki, S. Hagiwara,; T. Yoshino, Y. Arai, T. Yamamoto, "Polymer-Graphite Composite Loudspeaker Diaphragm," J. Audio Engineering Society, Vol. 29, Issue 10, pp. 720-725 (1981)

36. S. Takahashi,; T. Katoh,; S. Taguchi,; T. Watanabe, "Glass-Fiber and Graphite-Flake Reinforced Polyimide Composite Diaphragm for 
Loudspeakers," J. Audio Engineering Society, Vol. 31, Issue 10, pp. 723-728 (1983)

37. S. Taguchi, T. Watanabe, E. Takahashi, S. Takahashi, S. Tanaka, "SandwichConstruction Loudspeaker Diaphragm with Foamed High-Polymer and Carbon Fiber," J. Audio Engineering Society, Vol. 34, Issue 11, pp. 895-904 (1986)

38. G. W. Sioles, "Tweeter Design Considerations," J. Audio Engineering Society, Vol. 4, Issue 3, pp. 105-109 (1956)

39. H. Nakajima, M. Ugaji, H. Suyama, "Tweeter Using New Structure and New Material for Diaphragm," J. Audio Engineering Society, Preprint No. 1476, $63^{\text {rd }}$ Convention (1979)

40. J. A. M. Nieuwendijk, "Compact Ribbon Tweeter/Midrange Loudspeaker," J. Audio Engineering Society, Vol. 36, Issue 10, pp. 776-787 (1988)

41. J. Hayakawa, S. Iwakura, K. Yamazaki, S. Matsuoka, "Improvement in Dome Loudspeaker Characteristics by Using a Spherical-Wave-Front Horn Baffle," J. Audio Engineering Society, Vol. 36, Issue 7/8, pp. 575-584 (1988)

42. H. F. Olson, "The Action of a Direct Radiator Loudspeaker with a Non-Linear Cone Suspension System," J. Acoustical Society of America, Vol. 16, Issue 1, pp. 1-4 (1944)

43. R. V. L. Hartley, "The Production of Inharmonic Subfrequencies by a Loudspeaker," J. Acoustical Society of America, Vol. 16, Issue 3, pp. 206 (1945)

44. W. J. Cunningham, "Non-Linear Distortion in Dynamic Loudspeakers due to Magnetic Effects," J. Acoustical Society of America, Vol. 21, Issue 3, pp. 202-207 (1949) 
45. R. Larson, A. Adducci, "Transient distortion in loudspeakers," IRE Transactions on Audio, Vol. 9, Issue 3, pp. $79-85$ (1961)

46. D. R. Raichel, "Minimum Harmonic Distortion levels in Ideal Loudspeakers," J. Audio Engineering Society, Preprint No. $1285,58^{\text {th }}$ Convention (1977)

47. Jr. Sims, M. Travis, R. A. Greiner, "Loudspeaker Distortion Reduction," J. Audio Engineering Society, Preprint No. 2006, $74^{\text {th }}$ Convention (1983)

48. R. E. Werner, "Effect of a Negative Impedance Source on Loudspeaker Performance," J. Acoustical Society of America, Vol. 28, Issue 4, pp. 792 (1956)

49. G.L. Beers, H. Belar, "Frequency-Modulation Distortion in Loudspeakers," J. Audio Engineering Society, Vol. 29, Issue 5, pp. 320-326 (1981)

50. R. Allison, E. Villchur, "On the Magnitude and Audibility of FM Distortion in Loudspeakers," J. Audio Engineering Society, Vol. 30, Issue 10, pp. 694-700 (1982)

51. P.W. Klipsch, "Modulation Distortion in Loudspeakers," J. Audio Engineering Society, Preprint No. 562, $34^{\text {th }}$ Convention (1968)

52. P. W. Klipsch, "Loudspeaker Distortion," IEEE International Conference on Acoustics, Speech, and Signal Processing, Vol. 1, pp. 543- 546 (1976)

53. R. Small, "Direct-radiator loudspeaker system analysis," IEEE Transactions on Audio and Electroacoustics, Vol. 19, Issue 4, pp. 269- 281 (1971)

54. A. J. M. Kaizer, "Modelling of the Nonlinear Response of an Electrodynamic Loudspeaker by a Volterra Series Expansion," J. Audio Engineering Society, Vol. 35, Issue 6, pp. 421-433 (1987) 
55. W. Klippel, "Dynamical Measurement of Non-Linear Parameters of Electrodynamical Loudspeakers and their Interpretation," J. Audio Engineering Society, Vol. 38, Issue 12, pp. 944-955 (1990)

56. W. Klippel, "Modelling the Nonlinearities in Horn Loudspeakers," J. Audio Engineering Society, Vol. 44, Issue 6, pp. 470-480 (1996)

57. W. Klippel, "Nonlinear System Identification for Horn Loudspeakers," J. Audio Engineering Society, Vol. 44, Issue 10, pp. 811-820 (1996)

58. Y. Nomura, Y. Kajikawa, "Linearization of loudspeaker systems using MINT and Volterra filters," IEEE International Conference on Acoustics, Speech, and Signal Processing, Vol. 4, pp. 457- 460 (2005)

59. K. Lashkari, "A Novel Volterra-Wiener Model for Equalization of Loudspeaker Distortions," IEEE International Conference on Acoustics, Speech, and Signal Processing, Vol. 5, pp. 117- 120 (2006)

60. E. Villchur, "A Method of Testing Loudspeakers with Random Noise Input," J. Audio Engineering Society, Vol. 10, Issue 4, pp. 306-309 (1962)

61. G. W. Sioles, "Loudspeaker Measurements in Live Rooms," J. Audio Engineering Society, Vol. 11, Issue 3, pp. 203-206 (1963)

62. V. J. Kaminsky, "The Response of Loudspeakers to Tone Bursts," J. Audio Engineering Society, Vol. 13, Issue 2, pp. 119-123 (1965)

63. R. C. Heyser, "Acoustical Measurements by Time Delay Spectrometry," J. Audio Engineering Society, Vol. 15, Issue 4, pp. 370-382 (1967)

64. J. M. Berman, L. R. Fincham, "The Application of Digital Techniques to the Measurement of Loudspeakers," J. Audio Engineering Society. Vol. 25, Issue 6, pp. 370-384 (1977) 
65. L. R. Fincham, "Refinements in the Impulse Testing of Loudspeakers," J. Audio Engineering Society, Vol. 33, Issue 3, pp. 133-140 (1985)

66. J. D. Bunton, R. H. Small, "Cumulative Spectra, Tone Bursts, and Apodization," J. Audio Engineering Society, Vol. 30, Issue 6, pp. 386-395 (1982)

67. I. Nomoto, M. Iwahara, H. Onoye, "A Technique for Observing Loudspeaker Wave Front Propagation,” J. Audio Engineering Society, Vol. 24, Issue 1, pp. 9-13 (1976)

68. J. Hladky, "The Application of the Holography in Analysis of Vibrations of Loudspeaker Diaphragms," J. Audio Engineering Society, Vol. 22, Issue 4, pp. $247-250$ (1974)

69. P. A. Fryer, "Holographic Investigation of Speaker Vibrations," J. Audio Engineering Society, Preprint No. L-2, 50th Convention (1975)

70. F. J. M. Frankort, "Vibration Patterns and Radiation Behaviour of Loudspeaker Cones," J. Audio Engineering Society, Vol. 33, Issue 9, pp. 133140 (1985)

71. J. R. Tyrer, "The Use of TV Holography (ESPI) for Loudspeaker Chassis and Cabinet Modal Analysis," J. Audio Engineering Society, Preprint No. 2482, $82^{\text {nd }}$ Convention (1987)

72. G. Bank, G. T. Hathaway, "A Three-Dimensional Interferometric Vibrational Mode Display," J. Audio Engineering Society, Vol. 29, Issue 5, pp. 314-319 (1981)

73. D. J. Henwood,; C. J. C. Jones, P. A. Fryer, M. Worthington, "Finite Element Modelling of Loudspeaker Diaphragms and the Boundary Element Method for Evaluating Sound Pressure," J. Audio Engineering Society, Preprint No. 2449, $82^{\text {nd }}$ Convention (1987) 
74. J. R. Wright, "Automatic Vibration Analysis by Laser Interferometry," J. Audio Engineering Society, Preprint No. 2889, $88^{\text {th }}$ Convention (1990)

75. J. E. Weaver, Jr. Leach, W. Marshall, “Optical Measurement of Loudspeaker Driver Large Signal Displacement," J. Audio Engineering Society, Vol. 26, Issue 3, pp. 145-148 (1978)

76. J. N. Moreno, "Measurement of Loudspeaker Parameters Using a Laser Velocity Transducer and Two-Channel FFT Analysis," J. Audio Engineering Society, Vol. 39, Issue 4, pp. 243-249 (1991)

77. N. Harris, M. O. Hawksford, "The Distributed-Mode Loudspeaker (DML) as a Broad-Band Acoustic Radiator," J. Audio Engineering Society, Preprint No. $4526,103^{\text {rd }}$ Convention (1997)

78. M. Colloms, C. Ellis, "Diffuse Field Planar Loudspeakers in Multimedia and Home Theatre," J. Audio Engineering Society, Preprint No. 4545, $103^{\text {rd }}$ Convention (1997)

79. P. Mapp, M. Colloms, "Improvements in Intelligibility through the Use of Diffuse Acoustic Radiators in Sound Distribution," J. Audio Engineering Society, Preprint No. 4634, $103^{\text {rd }}$ Convention (1997)

80. H. Azima, N. Harris, "Boundary Interaction of Diffuse Field DistributedMode Radiators," J. Audio Engineering Society, Preprint No. 4635, $103^{\text {rd }}$ Convention (1997)

81. G. Bank, N. Harris, "The Distributed Mode Loudspeaker-Theory and Practice," AES UK Conference on Microphones \& Loudspeakers, The Ins \& Outs of Audio (MAL), Paper No. MAL-18 (1998) 
82. G. Bank, "The Intrinsic Scalability of the Distributed Mode Loudspeaker (DML)," J. Audio Engineering Society, Preprint No. $4742,104^{\text {th }}$ Convention (1998)

83. M. Roberts, "Exciter Design for Distributed-Mode Loudspeakers," J. Audio Engineering Society, Preprint No. $4743,104^{\text {th }}$ Convention (1998)

84. M. Colloms, V. Gontcharov, J. Panzer, V. Taylor, "Distortion Mechanisms of Distributed-Mode Loudspeakers (Compared with Direct Pistonic Radiators; Modelling, Analysis, and Measurement)," J. Audio Engineering Society, Preprint No. $4757,104^{\text {th }}$ Convention (1998)

85. M. Tashiro, G. Bank, M. Roberts, "A New Flat Panel Loudspeaker for Portable Multimedia," J. Audio Engineering Society, Preprint No. 4527, $103^{\text {rd }}$ Convention (1997)

86. M. Antila, H. Nykänen, K. Saarinen, "Multichannel Electromechanical Film Panel Loudspeaker," AES International Conference on Spatial Sound Reproduction, Paper No. 16-018 (1999)

87. H. Azima, J. Panzer, D. Reynaga. "Distributed-Mode Loudspeakers (DML) in Small Enclosures," J. Audio Engineering Society, Preprint No. 4987, $106^{\text {th }}$ Convention (1999)

88. J. A. S. Angus, "Distributed Mode Loudspeaker Radiation Mechanisms," J. Audio Engineering Society, Preprint No. $5164,108^{\text {th }}$ Convention (2000)

89. N. Harris, V. Gontcharov, M. O. Hawksford, "Measurement and Simulation Results Comparing the Acoustics of Various Direct Radiators in the Presence of a Dominant Specular Reflection," J. Audio Engineering Society, Preprint No. 5215, $109^{\text {th }}$ Convention (2000)

90. L. D. Copley, T. J. Cox, M. R. Avis, "Distributed Mode Loudspeaker Arrays," J. Audio Engineering Society, Preprint No. 5610, $112^{\text {th }}$ Convention (2002) 


\subsection{Introduction}

From the 1920's the perfect loudspeaker was judged in terms of the quality of the audio produced by operating the diaphragm like an ideal piston. Whether the loudspeaker diaphragm was driven from a moving armature, or later on a moving coil, the aim was to control the sound radiated by as pistonic a motion as possible.

Operating the diaphragm of a loudspeaker as a moving mass sets two requirements to maintain an even frequency response. The first is that the diaphragm has to be small enough to be approximated by a point source. A point source with constant acceleration (gained from its mass-controlled operation, with constant force) gives a flat frequency response.

The second requirement is that the whole diaphragm moves with the same acceleration, that is the diaphragm behaves like a piston. This presupposes a radiator size that is small compared to the wavelength being reproduced, and immediately leads to the requirement for two or more diaphragms to cover 3 or 4 octaves. It also means that the diaphragm has to be quite rigid over the frequency band it is trying to reproduce. More difficulties arise when, in a conventional pistonic loudspeaker, as much energy leaves the back of the diaphragm as the front, and since the source is coherent, it is reversed in phase. As a result, leaving the rear to radiate freely automatically produces a dipole radiator, with interference between back and front, and a loss of output at low frequencies. Although dipoles have been used in special circumstances for their audiophile qualities in respect of cabinet colouration, the onaxis sound pressure level falls sharply below the cut-off frequency. For all practical purposes then, this means the rear radiation inside a cabinet needs to be absorbed by some means. In addition, cabinet panel modes, standing waves and diffraction all make it difficult for a pistonic loudspeaker to work as an efficient broadband loudspeaker. 
Contrary to normal pistonic diaphragm behaviour, a Distributed Mode Loudspeaker (DML) is acoustic radiator, the electrical, mechanical and acoustical properties of which differ radically from conventional moving-coil transducers such that a new loudspeaker class is defined. A DML is distinguished by acoustic radiation that emanates from uniformly distributed, free bending wave vibration induced in a stiff, light panel and not from pistonic motion. This free and random motion of the flat panel diaphragm can reproduce sound that is not omni-directional at high frequencies as in the case of a conventional loudspeaker. However, the performance of a flat panel speaker is limited severely by the poor reproduction of the low frequency sound. The reason for such poor bass response is that, unlike the whole body movement of a conventional loudspeaker diaphragm, different parts of the panel in a flat panel loudspeaker vibrates independently. The independent vibration mechanism also results in a poor impulse response in a flat panel loudspeaker.

The limitations in both the conventional and the flat panel speaker technology have led to the need for a new design philosophy for loudspeakers. The new design for loudspeakers reduces the disadvantages associated with conventional and flat panel speakers without losing the advantages of both technologies. The new design philosophy is to introduce whole body motion in flat panel speakers. Instead of single exciter, the proposed miniature matrix array transducer system for loudspeaker employs numerous miniature transducers to vibrate coherently and produce sound effectively at the low and high range of the audio spectrum. This multi-actuator approach combines the advantages of conventional whole body motion with that of modern flat panel speakers. This novel configuration of the loudspeaker will enable selective excitation of transducers in the matrix array speaker and in this way there will be no need for a cross-over network. The novel miniature matrix array transducer speaker will produce non-directional high frequency response and also quality low frequency sound by implementing selective excitation and whole body vibration respectively. The co-ordinated vibration in the new loudspeaker will also enable good impulse response. 


\subsection{Electromagnetic Transducers}

Various actuation mechanisms such as piezoelectric, electrostatic, magnetostrictive and electromagnetic have been used for transduction in loudspeakers over the years. However, electromagnetic transducers, due to their high power output, have been the most effective in loudspeaker application. Recent studies [1] have shown that electromagnetic actuators can be even more effective and useful when scaled down. Most importantly magnet-magnet and magnet-iron interactions benefit immensely from a scale reduction while the force-to-volume ratio of magnet-current interactions remains unchanged. A magnet of a certain polarization, when scaled down, exerts the same field at a distance reduced by the same amount as the magnet itself [1]. This phenomenon has important consequences since the conductor that interacts with the magnet experiences the same force as before because of the constant field. In addition, the current carrying conductors are more efficient in terms of heat dissipation when scaled down. The joule losses that heat up the conductors are proportional to the volume but the heat dissipation is proportional to the surface area, therefore when a conductor is scaled down, the heat generation to heat dissipation ratio decreases [1]. The decrease in heating results in the increase of current density of conductors within the admissible thermal limits. Therefore electromagnetic actuators can be very useful in designing miniature transducers which can offer greater advantages over other actuation mechanisms. Figure 6.1[1] summarizes the general effects of a scale reduction of factor $\mathrm{k}$ on the mass and volume related forces interacting between the basic magnetic components (magnet, current, ferromagnetic material, and to a certain degree induction effects), for a constant current density.

It can be observed that the main magnetic interactions can benefit from a scale reduction. The chart also shows that in small-dimension devices, the most efficient magnetic interactions involve permanent magnets. The most important conclusions that can be derived from this interaction chart are that any electromagnetic device, having permanent magnets and interacting with currents, which is effective on a macroscopic scale remains as effective once miniaturized; interactions between permanent magnets are strongly improved by scale reduction and inductive effects are to be avoided. 
Figure $6.2[1]$ shows the effect of scale reduction on magnetic interactions, taking into account increased admissible current density. It is evident that a reduction in coil size results in the increase of admissible current density. It can also be shown from figure 6.2 that interactions with coils are improved by the increase in current density (within the admissible thermal limits) and permanent magnets are the key to efficient miniature transducers. The results clearly shows that a miniature transducer employing either micro coils and a permanent magnet or micro coils and an iron are the two most effective actuation mechanisms for loudspeaker application.

\begin{tabular}{|c|c|c|c|c|}
\hline $\begin{array}{c}\text { Reduction } \\
\text { factor } \\
1 / k\end{array}$ & magnet & current & iron & $\begin{array}{l}\text { induction } \\
E=\mathrm{d} \Phi / \mathrm{dt}\end{array}$ \\
\hline magnet & $\times k$ & & $x k$ & / $k$ \\
\hline current & & $/ \mathrm{k}$ & / k & $/ k^{2}$ \\
\hline
\end{tabular}

Figure 6.1: Magnetic interactions (per volume / per mass): Effects of scale reduction by a factor $\mathrm{k}[1]$

\begin{tabular}{|c|c|c|c|c|}
\hline $\begin{array}{c}\text { Reduction } \\
\text { factor } \\
1 / k\end{array}$ & $\begin{array}{c}\text { magnet } \\
\text { magnet }\end{array}$ & \begin{tabular}{c} 
current \\
\hline
\end{tabular} & $\times k_{i}$ & \\
\hline
\end{tabular}

Figure 6.2: Interactions (per volume / per mass): Effects of scale reduction by a factor $\mathrm{k}$ and of the increase in current density $\mathrm{k}_{\mathrm{i}}[1]$ 


\subsection{Feasibility Study}

\subsubsection{Current-Iron Transducer}

A solenoid transducer (Fig. 6.3) was observed for loudspeaker application as part of the feasibility study. The solenoid usually refers to a tube like coil only. When current passes through the solenoid, it generates a magnetic field around it. The magnetic field inside is much larger than it is outside and as a result considerable magnetic energy is stored in the interior. If a bar of magnetic material is placed at one end of the solenoid, it is drawn into the solenoid as the magnetic circuit tries to reduce the reluctance which is mostly made up by the air. The stored magnetic energy inside the solenoid is transformed to mechanical energy as a result of the plunger moving in. The actuator exploits the magnetic field in a solenoid for actuation purpose by providing an outer flux return path of magnetic material and an inner flux return path through a magnetically permeable plunger which is pulled into the coil when electric current passes through the winding. The coil in the solenoid actuator creates an MMF (Magneto-Motive Force) which drives flux through the plunger and then around the frame of the solenoid and then through the air-gap and back into the plunger. A solenoid actuator of this construction operates in one direction only and the plunger is returned only when the current is cut-off by some auxiliary means such as a spring $[2]$.

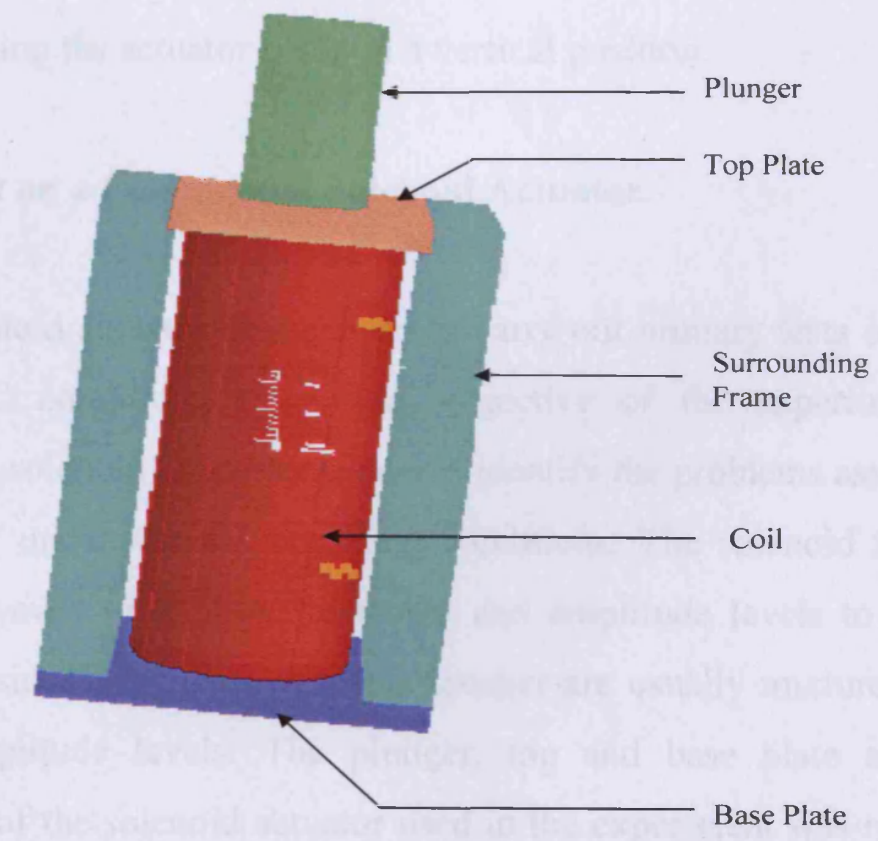

Figure 6.3: A three dimensional model of a solenoid transducer 
The force produced by the magnetic field can be explained using the following equation [3]:

$F_{\text {mag }}=\frac{\phi^{2}}{2 \cdot \mu_{0} \cdot A_{g}}=\frac{B_{g}{ }^{2} \cdot A_{g}}{2 \mu_{0}}$

Here $F_{m a g}$ is the magnetic force generated in the air-gap, $\phi$ is the magnetic flux, $B_{g}$ is the magnetic flux density in the air-gap, $A_{g}$ is the area of the air-gap and $\mu_{0}$ is the permeability of free space.

The overall force equation governing the solenoid actuator's behaviour can be given as follows [4]:

$m \cdot \frac{d v}{d t}=-c x+F_{m a g}+m g-F_{f r i c}-F_{\text {lim }}$

Here $v$ is the velocity, $t$ is the time, $c$ is the spring coefficient, $x$ is the displacement, $m$ is the mass of the plunger, $g$ is the gravity, $F_{\text {mag }}$ is the magnetic force, $F_{\text {fric }}$ is the friction force and $F_{\lim }$ is the limiting force. The above equation has been derived assuming the actuator being in a vertical position.

\subsubsection{Experiment on a Commercial Solenoid Actuator}

A commercial solenoid actuator was chosen to carry out primary tests on a solenoid actuator under AC condition. The initial objective of the experiment was to familiarise with the solenoid characteristics and identify the problems associated with a solenoid actuator under various operating conditions. The solenoid actuator was tested using sine waves of various frequency and amplitude levels to observe the plunger behaviour since the inputs of a loudspeaker are usually mixtures of various frequency and amplitude levels. The plunger, top and base plate and also the surrounding frame of the solenoid actuator used in the experiment was made of mild steel and the number of coil turns was 1120 . 
The displacement versus current results (Fig. 6.4) shows the limitations of solenoid actuators operating in the audio frequency region. At lower excitation current, the solenoid actuator produced linear displacements with current. But as the excitation current was increased, non-linearity was introduced in the output. The non-linearity was introduced because the base plate was getting highly magnetized due to high current and then failing to de-magnetise completely before next cycle started. The residual magnetism in the base plate resulted in the non-linear displacements of the plunger.

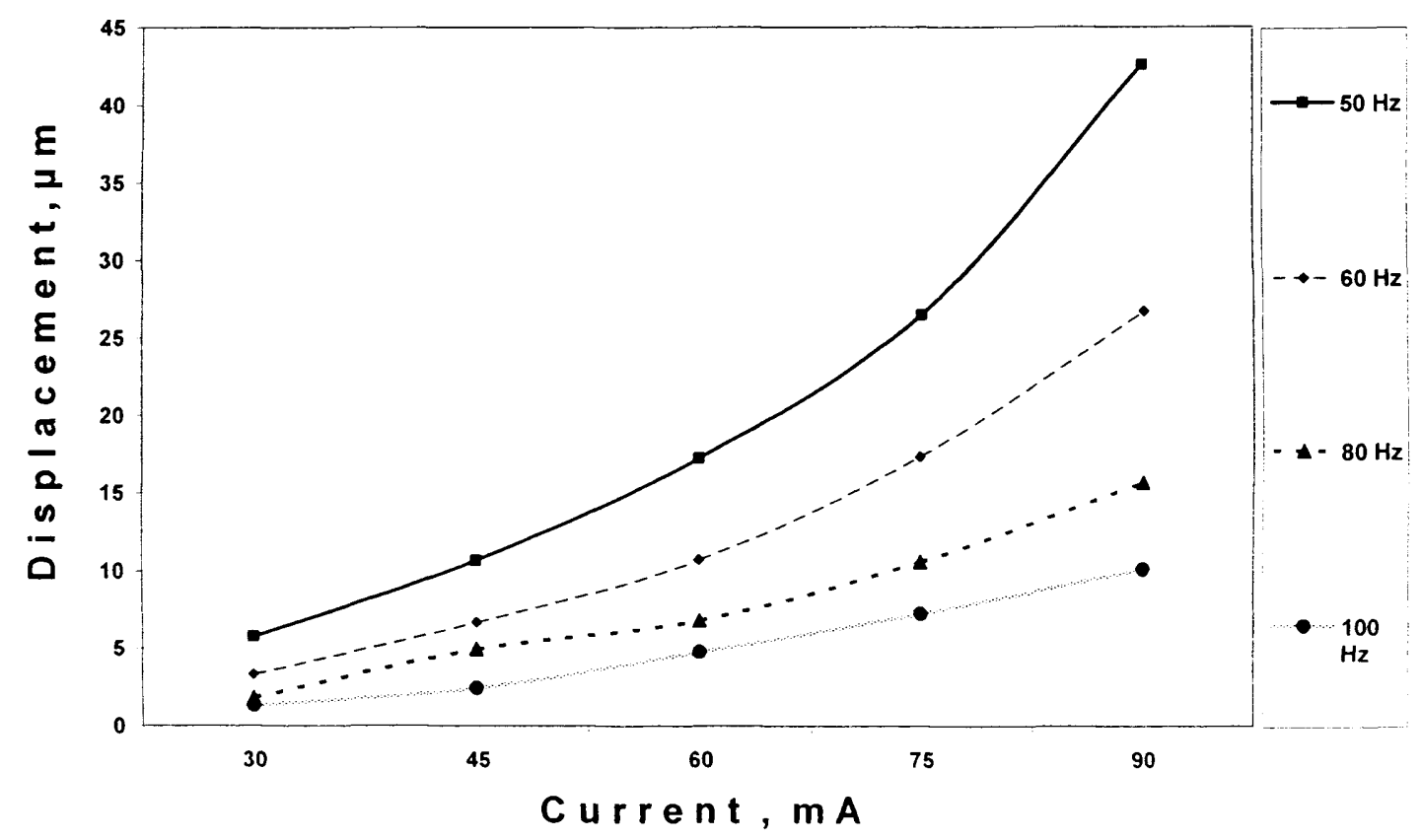

Figure 6.4: Displacement profile of the solenoid actuator for 1120 turns for various frequency and current levels

As the exciting current was increased further, especially above $150 m A(p-p)$, displacement reduced and the plunger exhibited only one way movement (Fig. 6.5). 


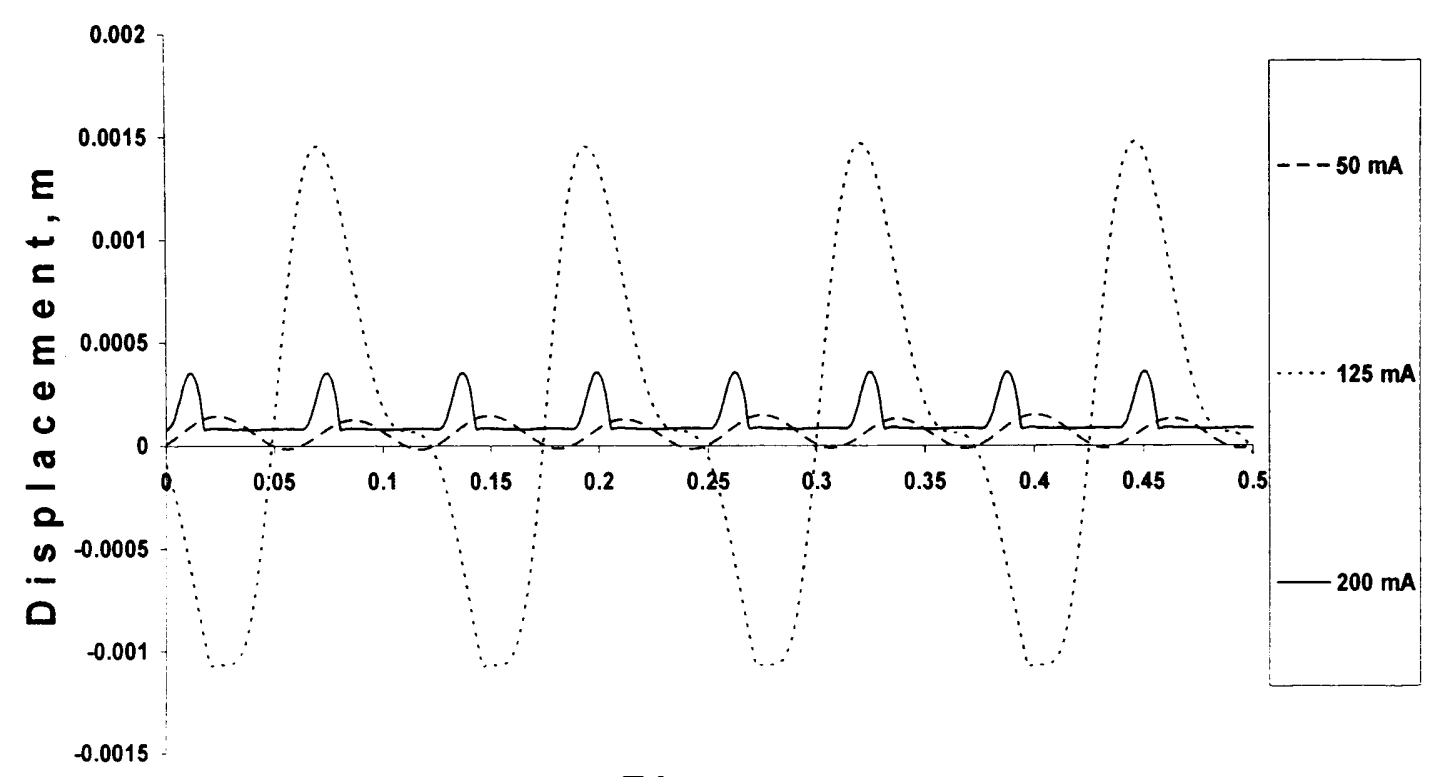

Time,s

Figure 6.5: Displacement of a solenoid actuator for 1120 turns for an excitation frequency of $20 \mathrm{~Hz}$ at different current levels

The reduction in displacement could be explained by the fact that at higher excitation currents, the coil resistance increased excessively due to overheating and hence received less current and consequently produced less force which resulted in low displacement of the plunger. The one way movement in the solenoid transducer at high currents can be explained by the fact that the electromagnetic force is unidirectional for a solenoid actuator. At higher excitation currents, the uni-directional force is greater and this results in the return spring getting fully compressed. This brings the plunger in touch with the base plate which is at a saturated state at higher excitation currents. The magnetically saturated base plate attracts the plunger and as a result the plunger gets magnetically attached to the base plate. However, during the negative half of an input cycle, the base plate demagnetizes itself partially and the plunger travels a small distance in the opposite direction before the next positive half of a cycle appears and the plunger gets magnetically attached to the base plate again.

The next part of the experiment was to change the number of turns and observe how it affected the solenoid behaviour. For this, the number of turns was almost halved and reduced to 600 . The solenoid actuator, before and after reducing the number of turns, 
was tested for higher frequency operation such as 200 and $300 \mathrm{~Hz}$. As expected, the solenoid actuator with a lower number of turns could operate in the high frequencies and produce displacement whereas the actuator with a higher number of turns could not produce any sinusoidal displacement at these frequencies. This result with the 600 turns solenoid was expected since fewer turns meant less impedance and lower impedance allowed higher frequency operation. However, the other limitations associated with the 1120 turns solenoid actuator was still applicable for the 600 turns solenoid actuator.

Another important disadvantage of using a solenoid actuator is the effect of the return spring on the actuator's performance. The return spring introduces instability in the system by producing unwanted oscillation. The resonant frequency of the spring, used in the commercial solenoid actuator, was calculated to be $25 \mathrm{~Hz}$ and as a result the primary resonant frequency along with different harmonics of it caused unwanted oscillation in the plunger motion. As a result of the return spring action, the impulse response of the solenoid transducer was also poor (Fig. 6.6) and not suitable for loudspeaker application.

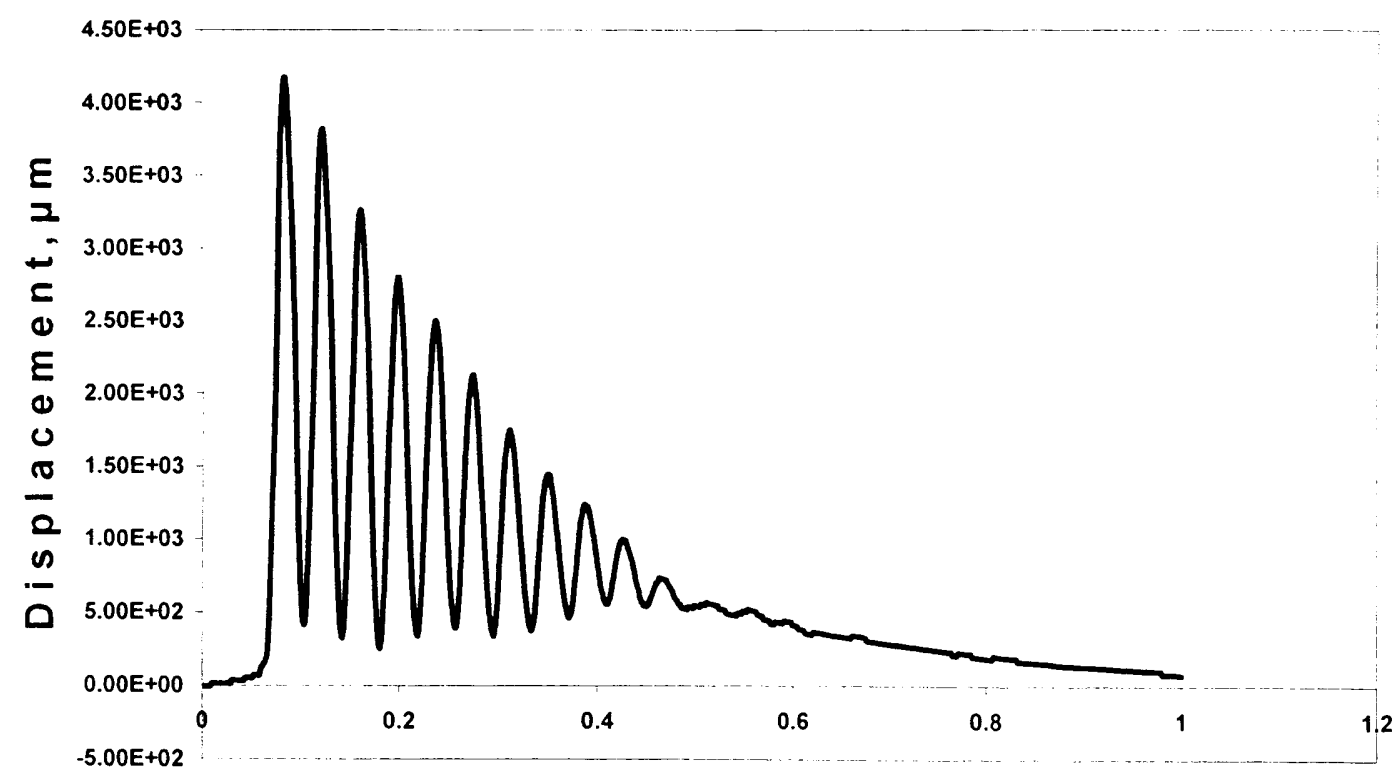

Time, $\mathbf{s}$

Figure 6.6: Impulse response of the Solenoid Actuator 


\subsubsection{FEM Simulation on Solenoid Actuator}

In recent days Finite Element Modelling (FEM) has widely been used for designing and understanding electromagnetic actuators. In this case, an electromagnetic design software (OPERA) [5] has been used to model the solenoid actuator and examine its behaviour. The solenoid actuator has been modelled with 1120 turns having 0.3 $\mathrm{amp} / \mathrm{mm}^{2}$ current density and the following analysis have been carried out: distribution of the flux contours within and around the solenoid, magnitude of the flux density within the solenoid, magnitude of the eddy current losses within the core and impact of the skin effect at higher frequencies.

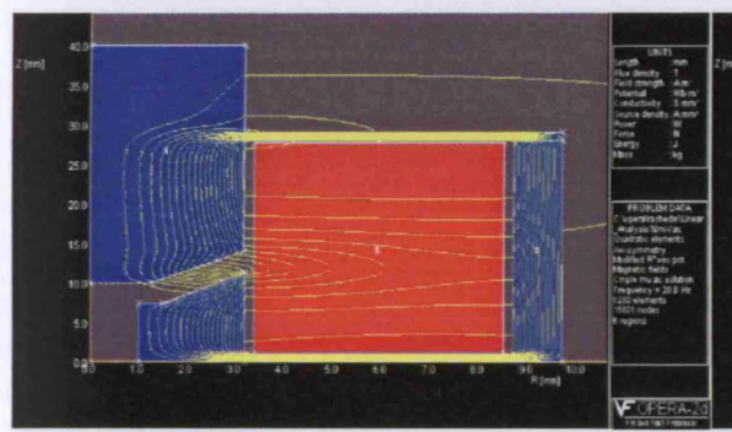

Figure 6.7: (a) Flux distribution at $20 \mathrm{~Hz}$

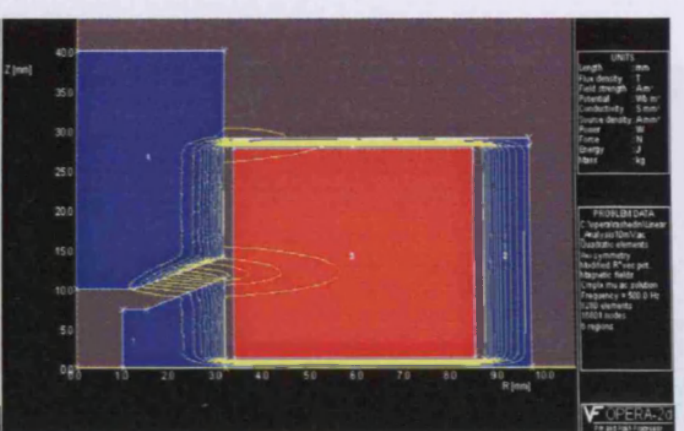

(b) Flux distribution at $500 \mathrm{~Hz}$

Figure 6.7 shows the flux distribution for the solenoid actuator at two different frequency levels. In the figure, the 'red region' is the coil, the 'blue region' on the left are the plunger and the bottom plate and the 'blue region' on the right is the surrounding frame of a solenoid actuator. Flux distributions were simulated for $20 \mathrm{~Hz}$ to $1000 \mathrm{~Hz}$. The simulation results showed the impact of eddy current effect at high frequencies. Flux distribution at $20 \mathrm{~Hz}$ (Fig. 6.7 a) showed the flux lines were uniformly spread over the plunger area and the surrounding frames. However at 500 $\mathrm{Hz}$, as shown in the simulation (Fig. $6.7 \mathrm{~b}$ ), the flux lines were reduced and were densely concentrated on the edges of the plunger and the surrounding areas. This was because the eddy current effect in the magnetic material increased at higher frequencies and limited the flux passing through the material.

Figure 6.8 showed the current density at different parts of the solenoid actuator in two different frequency levels. In this figure (Fig. 6.8) the red region denotes the coil in the actuator and it can be seen from the simulation result that at $20 \mathrm{~Hz}$, the current 
density was highest in the coil and was very low in the surrounding materials as expected, but as the frequency was increased current density in the magnetic materials started to increase and concentrated at the edges. These results from the FEM simulations showed the effects of eddy current and skin effect at high frequencies. The increase in current density in the material at $500 \mathrm{~Hz}$ was due to the eddy currents at high frequencies. Also because of the eddy currents in the coil at higher frequency, skin effect was introduced and as a result the current was concentrated mostly at the surface of the coil. The eddy current loss at high frequency is a major problem in the operation of electromagnetic actuators and a solenoid actuator is no exception in this case. The skin effect also makes a significant negative impact on the electromagnetic force creation.

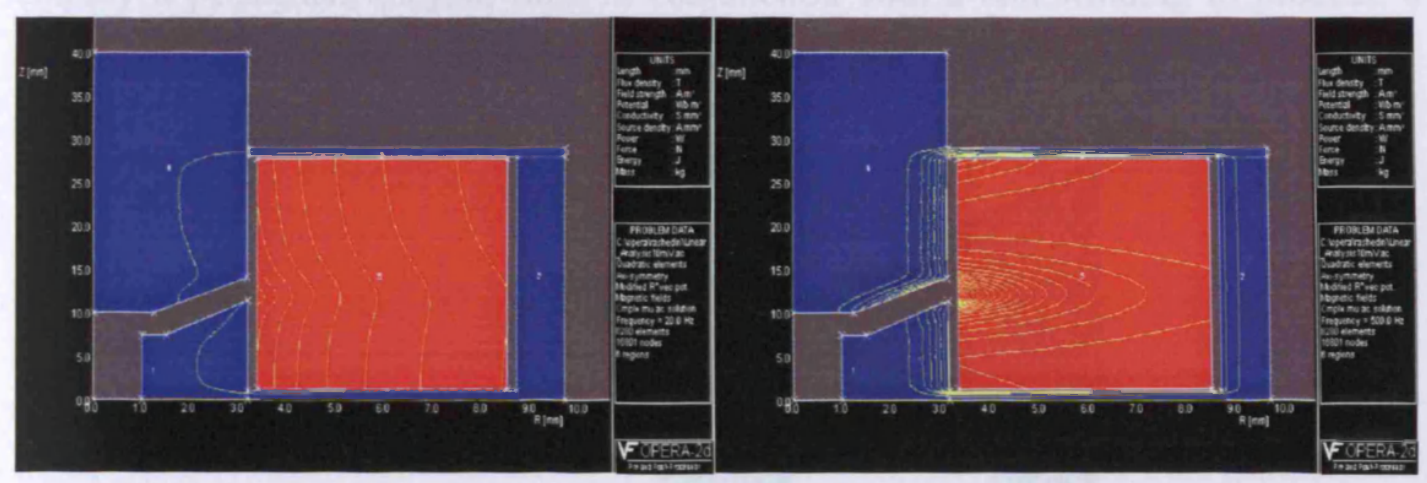

Figure 6.8: (a) Current distribution at $20 \mathrm{~Hz}$

(b) Current distribution at $500 \mathrm{~Hz}$

The solenoid actuator can be very useful in applications where simplicity and low cost are of prime importance but the actuator suffers from various limitations as seen from practical tests and FEM simulation results. The surrounding iron frame introduces hysteresis in the magnetic circuit, produces eddy current loss and skin effect and all these result in distorted output. Moreover, the variable reluctance of the magnetic circuit produces non-linear force-current relationship which is not ideal for a loudspeaker application. 


\subsubsection{Current-Magnet Transducer}

The experiment results in section 6.3 .1 for the solenoid actuator showed the nonlinear response of the plunger movement due to hysteresis, residual magnetism, spring and other limiting factors. In order to make a comparative study between a currentiron and a current-magnet transducer configuration, a conventional voice coil actuator was chosen to observe the properties of the latter. A moving voice coil transducer from a flat panel speaker (Packard Bell Company, Model No. 2.1) was tested for different frequencies and excitation currents using the laser vibrometer.

Voice coil transducers (Fig. 6.9) are versatile direct drive, hysteresis-free, noncommuted limited motion servo motors with linear control characteristics. They employ a permanent magnet field in conjunction with a coil winding to produce a force proportional to the current applied to the coil [6].

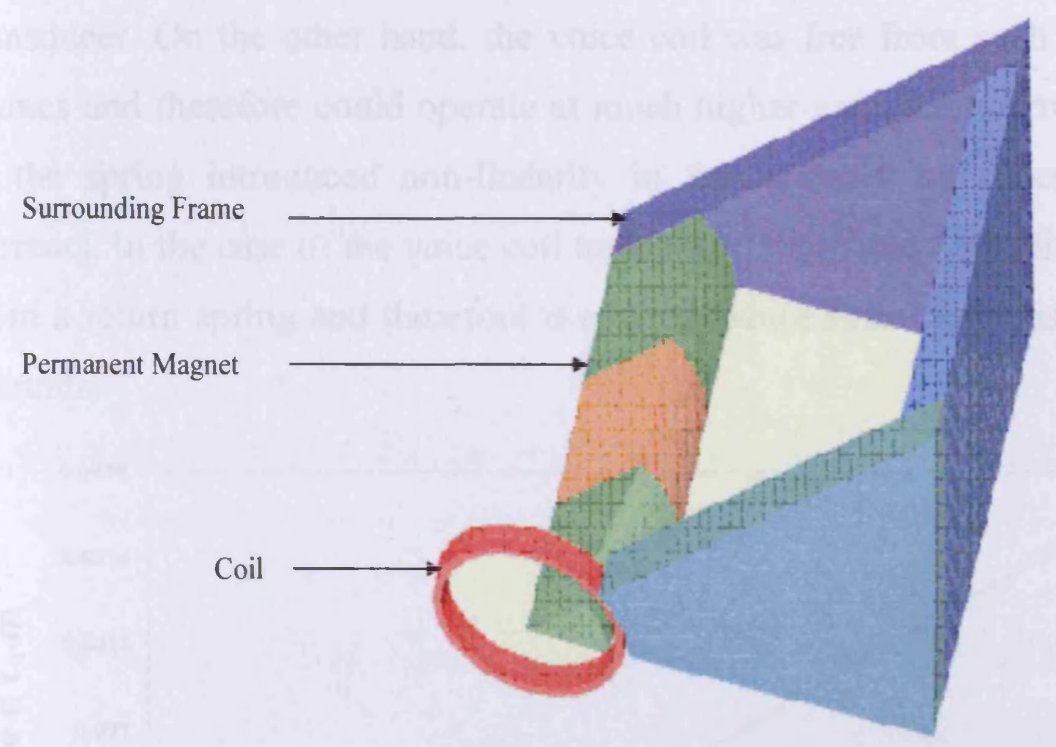

Figure 6.9: A three dimensional model of a voice coil transducer

The behaviour of a voice coil transducer can be explained by reference to the classical physics problem of a current carrying wire supported in a magnetic field. Force $F$ acting on the coil in the voice coil transducer is developed according to the following equation [6]:

$$
F=B i l
$$


where the magnetic field strength is $B$, the current carried by the wire is $i$, and the length of the portion of wire cut by the field is $l$. The force developed is perpendicular to both the magnetic field, and to the current flowing in the wire.

\subsubsection{Experiment on a Commercial Voice Coil Actuator}

In the case of a solenoid actuator, the displacement versus current relationship was non-linear for higher excitation currents but for a voice coil transducer, the experimental results (Fig. 6.10) showed that the displacement versus current relationship is linear even for high excitation currents. This clearly shows the difference between the two different transducer configurations. At higher current levels, the performance of a solenoid was severely limited due to the hysteresis and residual magnetism in the surrounding frame and the base plate of the solenoid transducer. On the other hand, the voice coil was free from such effects of the iron frames and therefore could operate at much higher excitation currents. The stiffness in the spring introduced non-linearity in the solenoid transducer at high driving currents. In the case of the voice coil transducer, there was no additional non-linearity from a return spring and therefore it could produce linear response at higher driving currents.

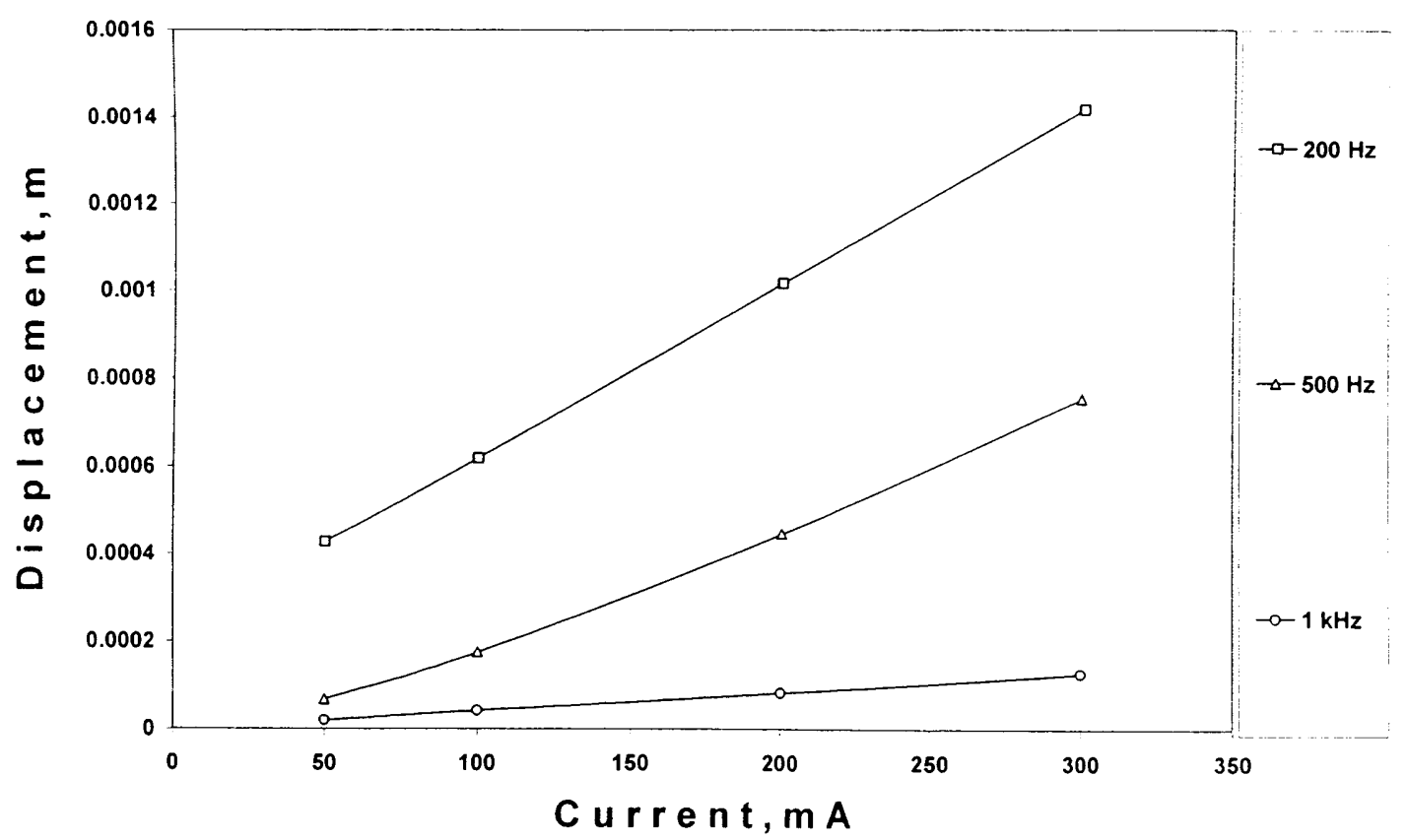

Figure 6.10: Displacement versus current relationship for the voice coil transducer 
Similar linear undistorted responses were obtained for voice coil transducer at higher frequencies. At high frequencies, the solenoid transducer could only produce distorted outputs due to eddy currents, stiffness of the spring and residual magnetism in the surrounding iron frames. Whereas in the case of a voice coil transducer, there was no iron surroundings and hence the effects associated with iron frames did not affect the behaviour of a voice coil transducer at higher frequencies.

Voice coil transducers offer excellent control characteristics where linear actuation is required over short distances. Comparison of the displacement characteristics for voice coil devices and solenoid actuators shows the difference between these devices. The linear displacement versus current characteristic exhibited by the voice coil motor lends this to applications requiring precise control of displacement, whereas the nonlinear displacements in solenoid devices make these devices difficult to control.

The voice coil motor can develop force in either direction by reversing polarity of the excitation. Typically in a solenoid, a spring is required to produce force in the return direction, this spring force subtracts from the magnetic force developed so reducing force and speed in the energised direction. Combined with low inductance this makes possible cycle times less than $1 \mathrm{~ms}$ in certain cases, typically an order of magnitude faster than solenoid devices. Since the voice coil assembly is composed entirely of non-ferromagnetic materials, therefore there are no cogging forces between the coil and magnet assembly and this act as an advantage for loudspeaker application.

Another important characteristic explored during the feasibility study is that, for a solenoid transducer, it is impossible to achieve even a $60 \mathrm{~g}$ (at $500 \mathrm{~Hz}$ ) acceleration due to the magnetic hysteresis in the surrounding iron frame. Compared to the frequency limitation of a solenoid actuator, a voice coil or a current-magnet combination can easily produce non-distorted output at $20 \mathrm{kHz}$ or above.

Therefore, it can be concluded that a current-magnet combination is more suitable for loudspeaker applications compared to a current-iron combination as far as frequency range and linearity is concerned. Even at low frequencies, the use of a return spring action in a solenoid actuator makes it unsuitable for loudspeaker application. Moreover, non-linearities introduced in the solenoid transducer at higher excitation 
currents also make it difficult to apply in loudspeakers. On the other hand, the nondistorted frequency response of a current-magnet combination at various excitation currents over the whole audio frequency range showed that this configuration is the most suitable for loudspeaker application.

\subsection{Design of the Novel Transducer Speaker}

An innovative miniature transducer for the proposed loudspeaker was designed, modelled and built for analysis. The novel miniature electromagnetic transducer speaker (Fig. 6.11) was designed and developed using the principle of two interacting magnetic fields. This novel transducer speaker, having a current-magnet combination, consisted of a permanent magnet placed on a high permeability amorphous magnetic ribbon which acted as the panel for the speaker. In addition, a copper wire coil attached to another amorphous magnetic ribbon supplied the drive field and flux closure respectively.

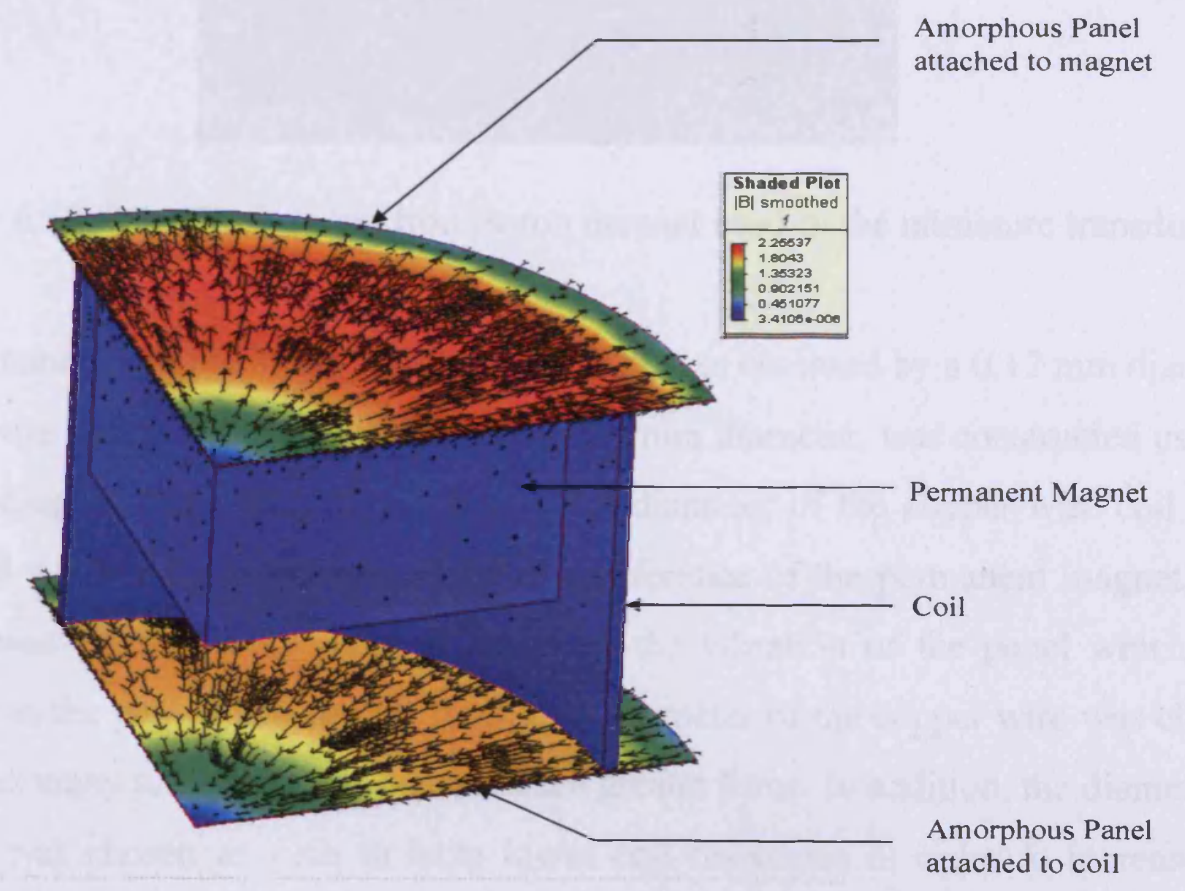

Figure 6.11: A quarter model of the novel transducer showing flux density and flux distribution 
The permanent magnet was a Neodymium Boron Iron magnet (rare-earth magnet) (Fig. 6.12), $6 \mathrm{~mm}$ in diameter, $2 \mathrm{~mm}$ in height and oriented normal to the attached surface. The Neodymium Iron Boron magnet is a powerful magnet made of a combination of neodymium, iron, and boron $-\mathrm{Nd}_{2} \mathrm{Fe}_{14} \mathrm{~B}$. Neodymium Iron Boron magnets have a Tesla level which is typically 2-4 times higher than standard Alnico magnets and they are ideal for loudspeaker applications with a working range up to $100^{\circ} \mathrm{C}$. The rare earth magnet was chosen for the novel miniature loudspeaker application because these magnets have very high energy product $(\mathrm{BH})$ in comparison to their mass and has good resistance to external demagnetization fields due to its high intrinsic coercive force.

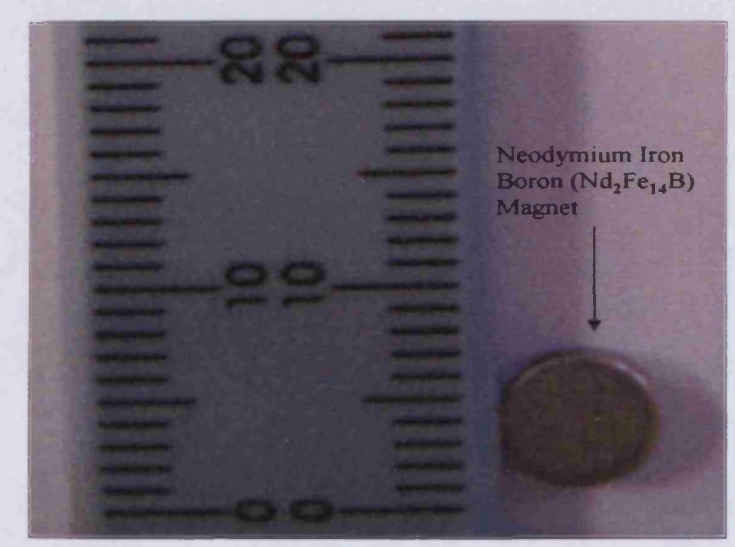

Figure 6.12: The Neodymium Iron Boron magnet used in the miniature transducer

The permanent magnet in the transducer speaker was enclosed by a $0.12 \mathrm{~mm}$ diameter copper wire coil. The core-less coil, having a $7 \mathrm{~mm}$ diameter, was constructed using a coil winding machine (Fig. 6.13). The $7 \mathrm{~mm}$ diameter of the copper wire coil (Fig. 6.14) left a $0.5 \mathrm{~mm}$ air-gap around the circumference of the permanent magnet. The air-gap was necessary for creating space for the vibration of the panel which was attached to the permanent magnet. The small diameter of the copper wire was chosen to have as many turns as possible to produce greater force. In addition, the diameter of the coil was chosen as such to have lower coil resistance in order to increase the maximum permissible current in the coil. 


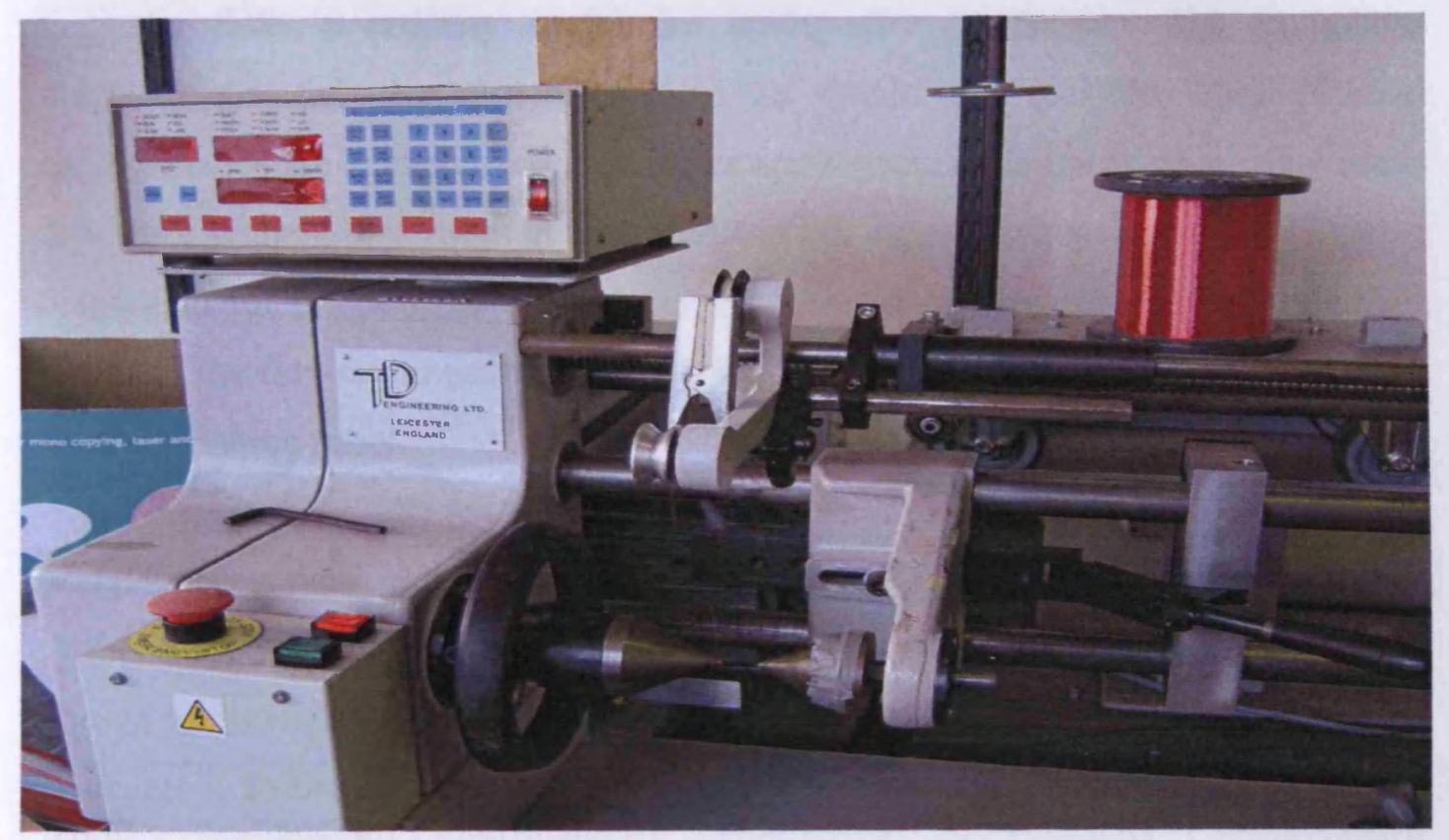

Figure 6.13: Coil winding machine used to construct the miniature core-less coil

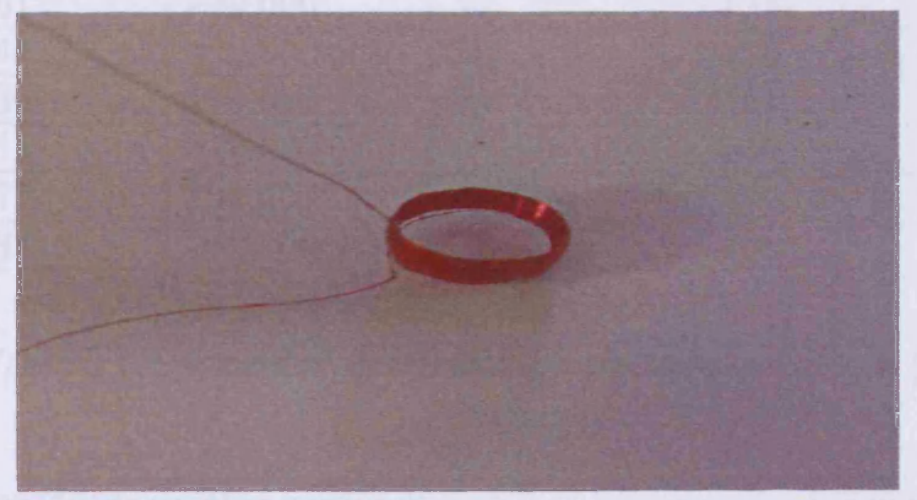

Figure 6.14: The miniature coil for the transducer speaker

The panels for the transducer speaker were made of amorphous ribbons which were iron based magnetic alloys with high saturation induction and high permeability. The magnetic alloy, known as Metglas, does not have crystalline structure like other magnetic materials. All the atoms in an amorphous metal are randomly arranged, thus giving it a higher resistivity value than that for crystalline counterparts [3]. Amorphous alloys are prepared by rapid cooling method and this fast cooling does not give the atoms enough time to rearrange into stable crystalline form. The amorphous alloys are magnetically soft (lower coercivity, lower core loss, higher permeability) due to the lack of the crystalline structure. The high resistance in the amorphous magnetic materials leads to low losses by eddy currents when subjected to alternating 
magnetic field, a property useful for loudspeaker application. The amorphous magnetic materials are also very light in weight and flexible. Such physical characteristics are suitable for loudspeaker application since the speaker panel needs to vibrate without distortions at high frequencies. Apart from working as a vibrating diaphragm for the miniature transducer speaker, the amorphous magnetic materials provided the flux return path for the magnetic circuit and also the return mechanism for the vibrating panel.

Metglas has some variations based on the alloys that make the amorphous material (Table 6.1). For the miniature transducer speaker application, Metglas $2605 \mathrm{CO}$ was chosen for its High saturation induction, square $\mathrm{BH}$ loop, and low coercivity.

\begin{tabular}{|l|l|l|l|l|l|}
\hline $\begin{array}{l}\text { Magnetic } \\
\text { Alloy }\end{array}$ & $\begin{array}{l}\text { Saturation } \\
\text { Induction } \\
(\text { Tesla })\end{array}$ & $\begin{array}{l}\text { Maximum DC } \\
\text { Permeability } \\
(\mu)\end{array}$ & $\begin{array}{l}\text { Saturation } \\
\text { Magnetost- } \\
\text { riction }(\mathrm{ppm})\end{array}$ & $\begin{array}{l}\text { Electrical } \\
\text { Resistivity } \\
(\mu \Omega-\mathrm{cm})\end{array}$ & $\begin{array}{l}\text { Curie } \\
\text { Temperature } \\
\left({ }^{\circ} \mathrm{C}\right)\end{array}$ \\
\hline 2605SA1 & 1.56 & 45,000 & 27 & 130 & 395 \\
\hline 2605SC & 1.61 & $>40,000$ & 30 & 135 & 370 \\
\hline $2605 \mathrm{~S} 3 \mathrm{~A}$ & 1.41 & $>20,000$ & 20 & 138 & 358 \\
\hline $\mathbf{2 6 0 5 C O}$ & $\mathbf{1 . 8 0}$ & $\mathbf{1 2 0 , 0 0 0}$ & $\mathbf{3 5}$ & $\mathbf{1 2 3}$ & $\mathbf{4 1 5}$ \\
\hline $2714 \mathrm{~A}$ & 0.57 & $>80,000$ & $<<1$ & 142 & 225 \\
\hline $2705 \mathrm{M}$ & 0.77 & 290,000 & $<<1$ & 136 & 365 \\
\hline $2826 \mathrm{MB}$ & 0.88 & $>50,000$ & 12 & 138 & 353 \\
\hline
\end{tabular}

Table 6.1: Physical properties of the amorphous ribbons [7]

In the novel transducer speaker configuration, two amorphous ribbons were employed as the top and the bottom panel. One of these magnetic layers was attached to the permanent magnet and the other was attached to the coil surrounding the permanent magnet. In order to the keep the permanent magnet and the magnetic panel attached to the coil apart, a minimum distance between the two was required. This 'minimum' distance was the deciding factor for determining the number of turns of the coil and hence the minimum size of the transducer speaker. A full schematic diagram of the novel transducer speaker is shown in figure 6.15 .

The overall size of each pixel was $8.5 \times 8.5 \times 4.5 \mathrm{~mm}$. The operation characteristics of this novel transducer have been observed and analysed both by simulation and practical experiments. The proposed new loudspeaker was developed using these novel actuators in a matrix configuration. 


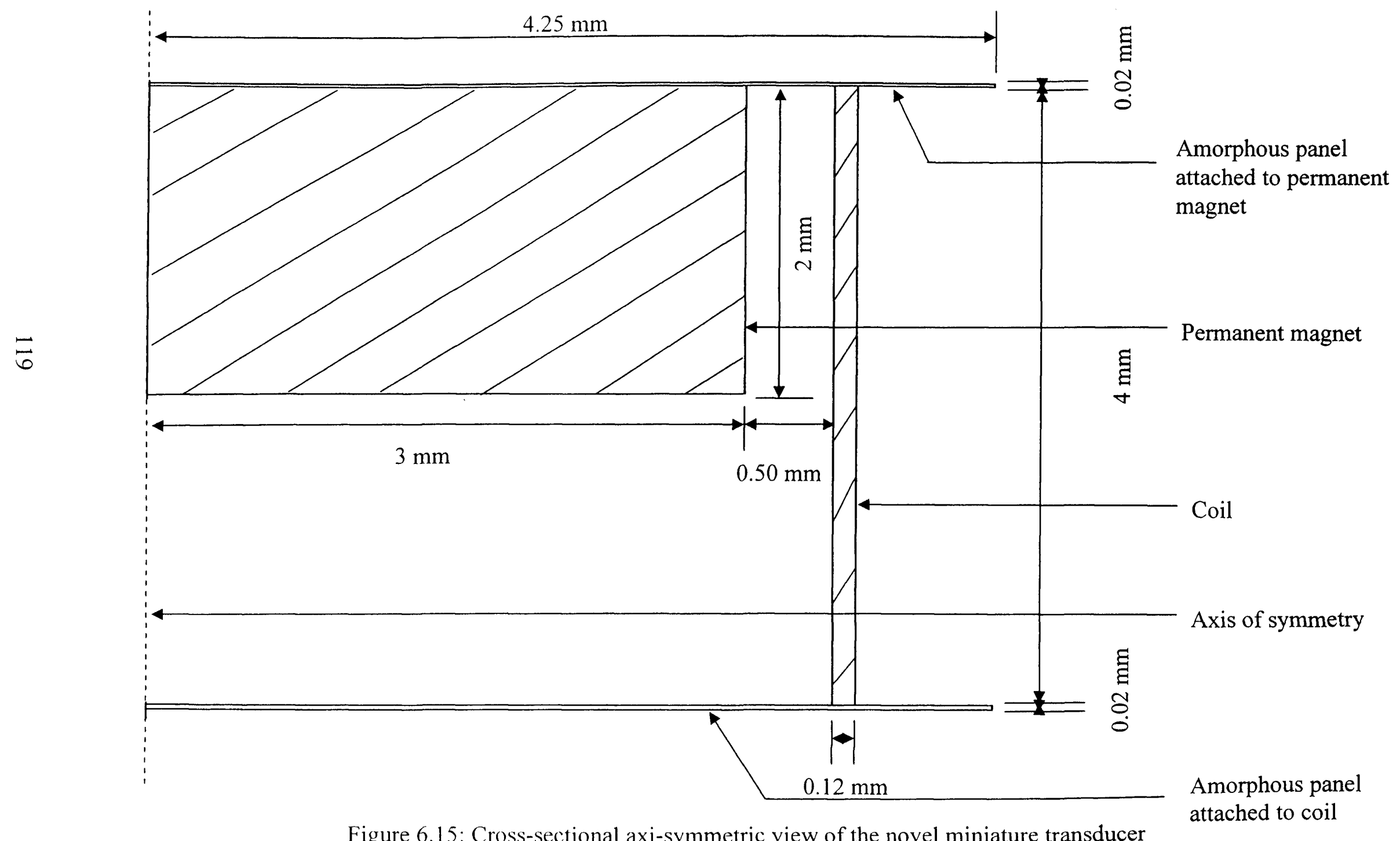




\subsection{Operation Principle}

For the single cell transducer, the upper and the lower magnetic material together with the permanent magnet (Nd-Fe-B) form a magnetic circuit. The excitation alternating current in the coil generates an alternating magnetic field that interacts with the static magnetic field of the permanent magnet. The interaction between the two fields causes an 'attract' and 'repel' action that translates to mechanical vibration of the panel. The instantaneous force that governs the displacement of the panel in a moving coil transducer is given by the following equation [8].

$$
F=B i l \sin \theta
$$

here $B$ is the magnetic flux density in the air gap due to the permanent magnet, $i$ is the instantaneous excitation current, $l$ is the length of the coil and $\theta$ is the angle of interaction between the two magnetic fields.

Simulation tools 'MAGNET' [8] and 'OPERA' [5] were used to characterize the transducer's magnetic performance. For a single cell transducer, under static condition, the simulations showed the flux distribution, flux density at various parts and the force distribution.

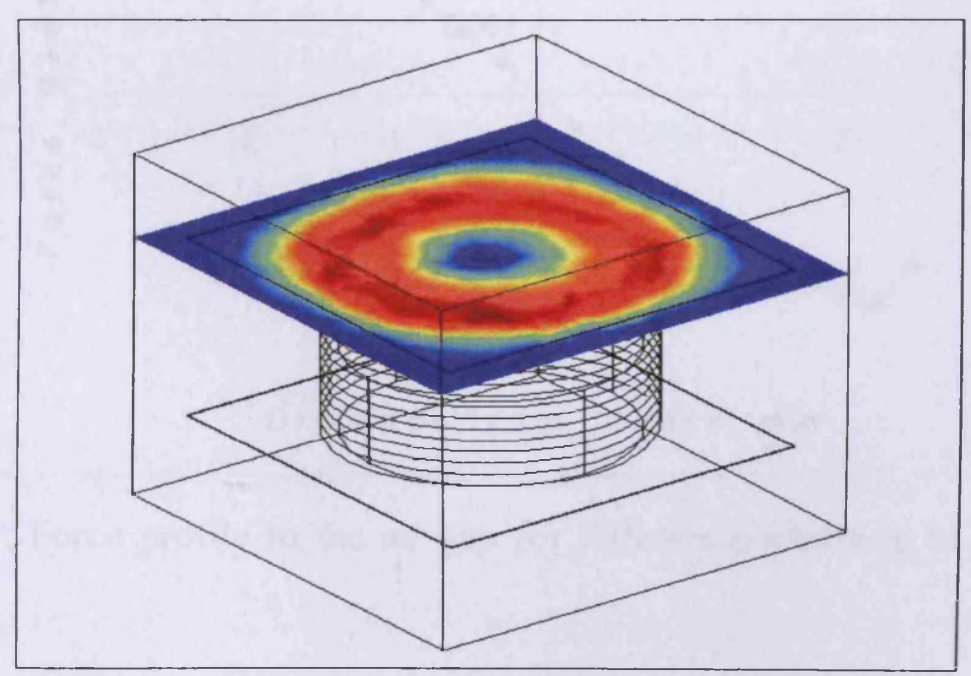

Figure 6.16: A three dimesional model of the novel transducer speaker showing flux density in the upper panel. 
The flux distribution of the transducer showed the flux circulating between the upper and the lower panel. The flux from the magnet flows through the upper panel and then returns via the lower panel of the transducer. There is a considerable amount of flux leakage due to the air gap present between the two panels. However, a minimum airgap was required to separate the permanent magnet from the lower panel. The simulation (Fig. 6.16) showed that the concentration of the flux on the upper panel increases from the centre towards the periphery of the magnet. This increase in flux density was due to the coil flux adding up to the flux from the permanent magnet.

The positioning of the permanent magnet within the magnetic circuit determines the magnitude of the force that vibrates the diaphragm. Therefore the magnet was placed at different positions within the air-gap to observe the force profile. The simulation result (Fig. 6.17) showed the ideal placement of the magnet for maximum force. It should be noted that at a certain position i.e. at the middle, the interacting magnetic fields cancel each other and this cancellation results in a null force as seen in the following force profile.

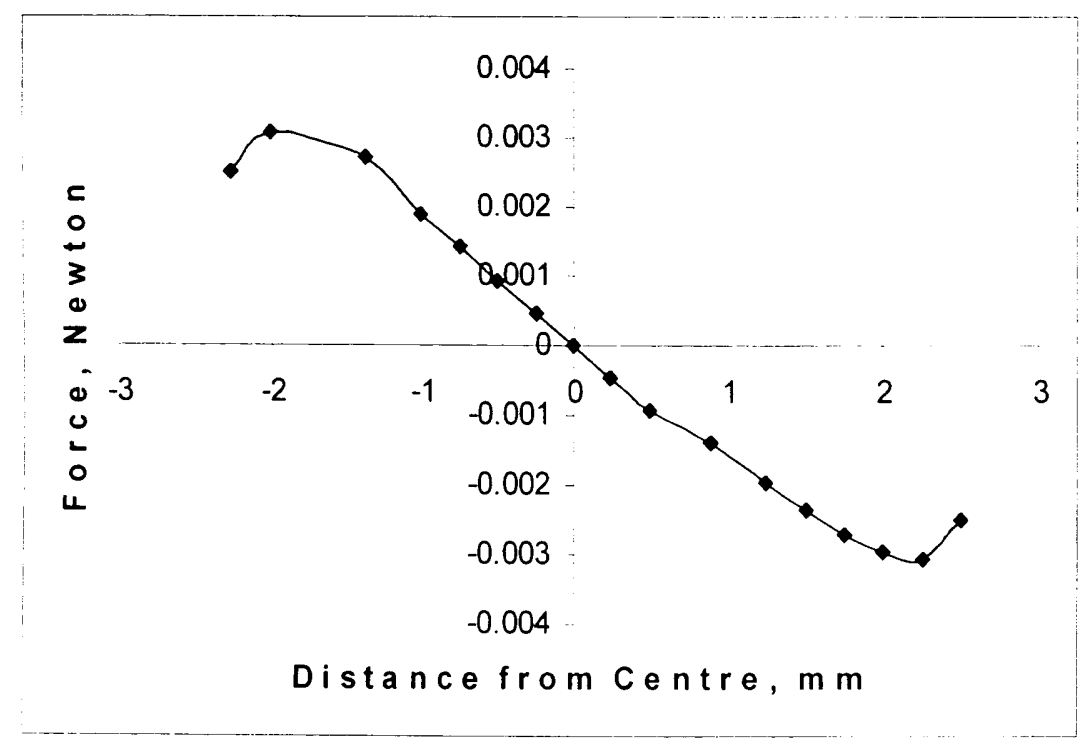

Figure 6.17: Force profile in the air gap for different positioning of the permanent magnet 


\subsection{Development of the Novel Loudspeaker}

Initially, three different device configurations of the miniature transducer speaker were built and measurements have been carried out on them in order to optimise the device. The three different configurations were:
(i) Moving Magnet
(ii) Moving Coil and
(iii) Moving Magnet with a Non-magnetic Bottom Layer

A moving magnet (Fig. 6.18) configuration of the miniature transducer speaker is the one in which the panel attached to the permanent magnet vibrates and the other panel that is attached to the coil remains static. Since in this configuration only the panel attached to the magnet vibrates, it is called a moving magnet configuration.

A moving coil (Fig. 6.19) configuration of the miniature transducer speaker, on the other hand, is the one in which the panel attached to the coil vibrates and the other panel that is attached to the permanent magnet remains stationary. In this device configuration, the coil along with the panel attached to it vibrates and hence it is called a moving coil configuration.

A moving magnet with a non-magnetic bottom layer (Fig. 6.20) configuration of the device has the same vibration mechanism as the moving magnet arrangement but without the stationary amorphous layer that remains attached to the coil in the transducer system. Instead of an amorphous ribbon, a non-magnetic material was used as the bottom layer of the transducer speaker.

Apart from the above device configurations of the transducer speaker, at a later stage, a miniature transducer speaker with non-magnetic diaphragm (moving magnet) was also tested for understanding the effects and advantages of a magnetic panel. 


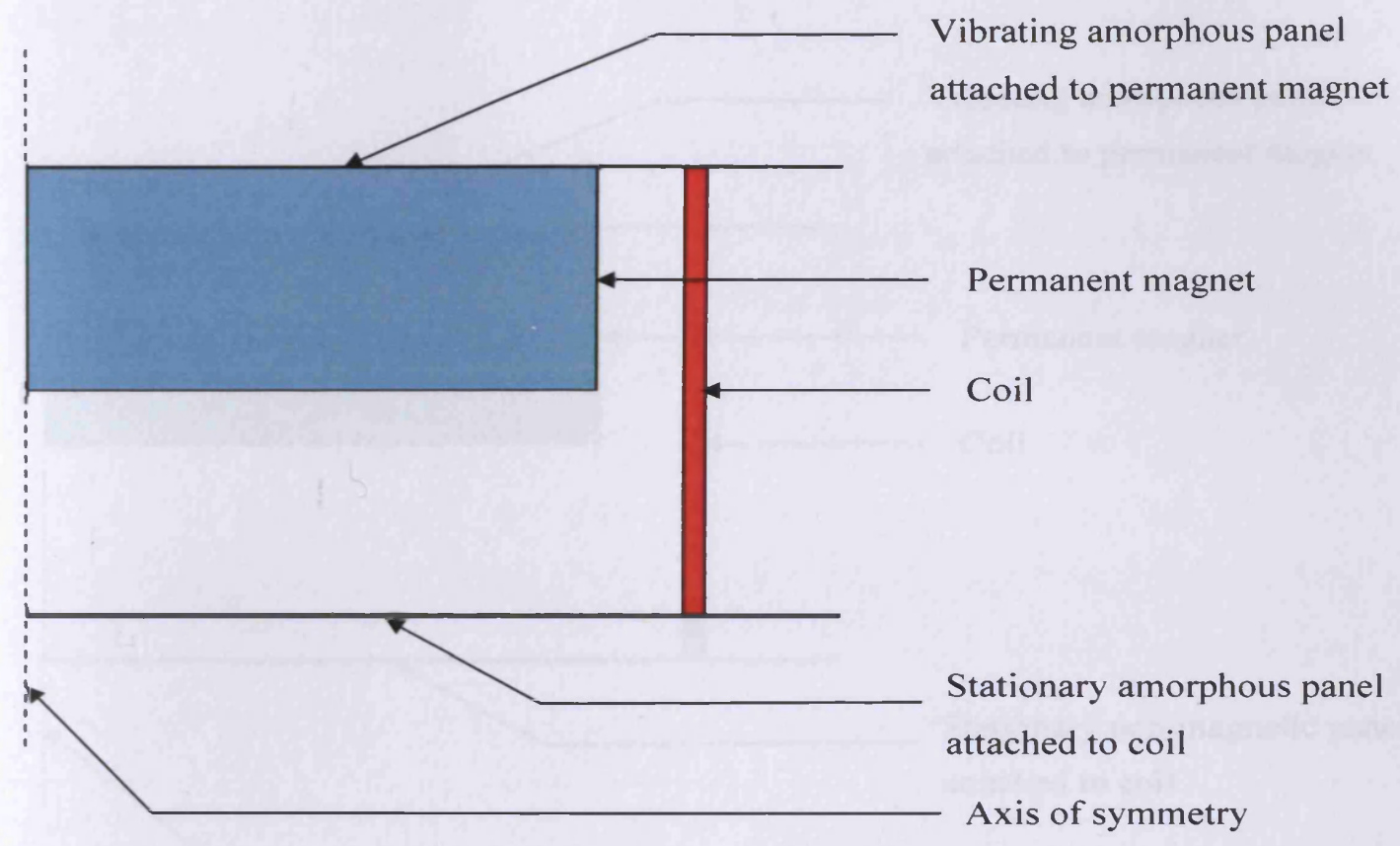

Figure 6.18: An axi-symmetric model of the transducer speaker showing the moving magnet configuration of the device

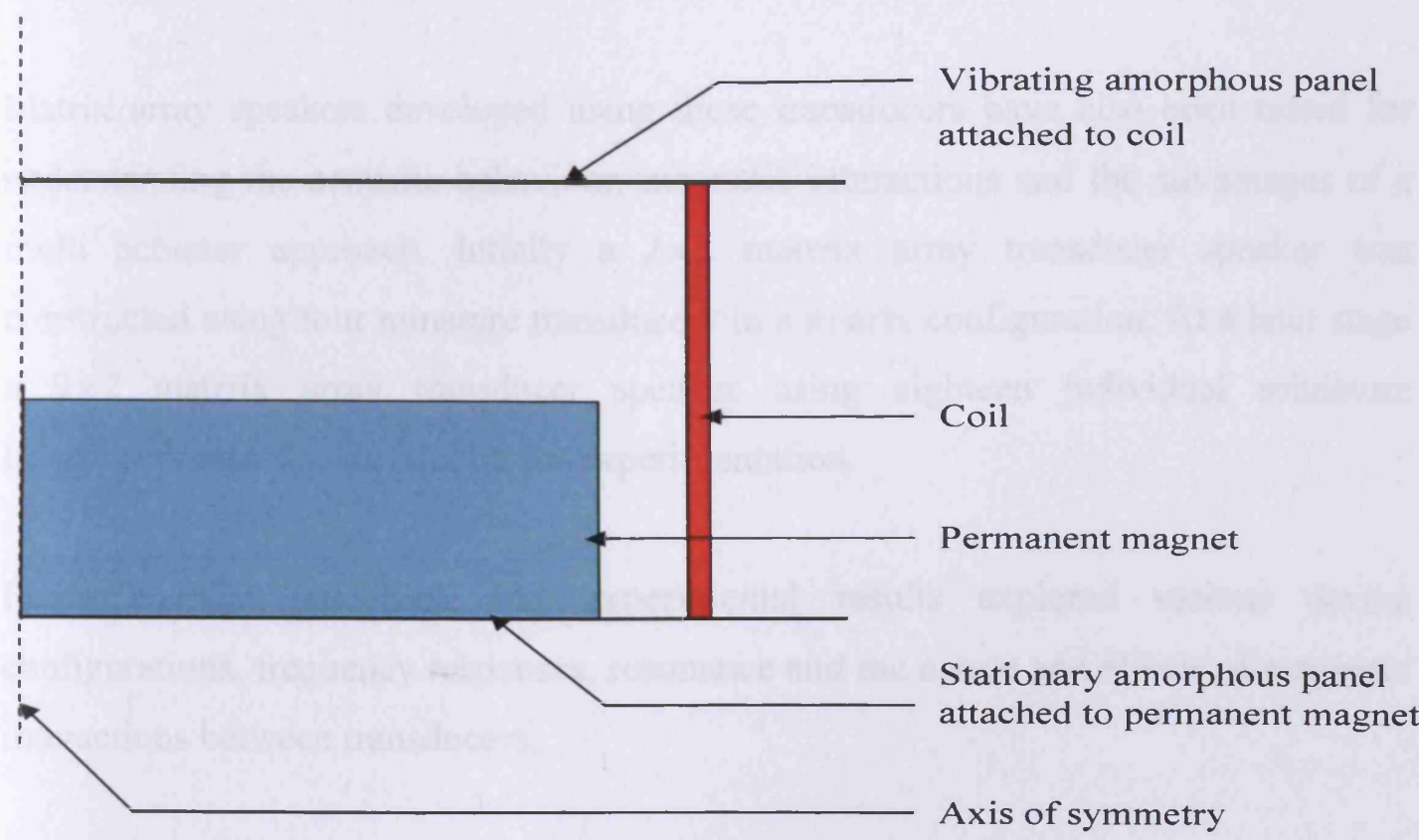

Figure 6.19: An axi-symmetric model of the transducer speaker showing the moving coil configuration of the device 


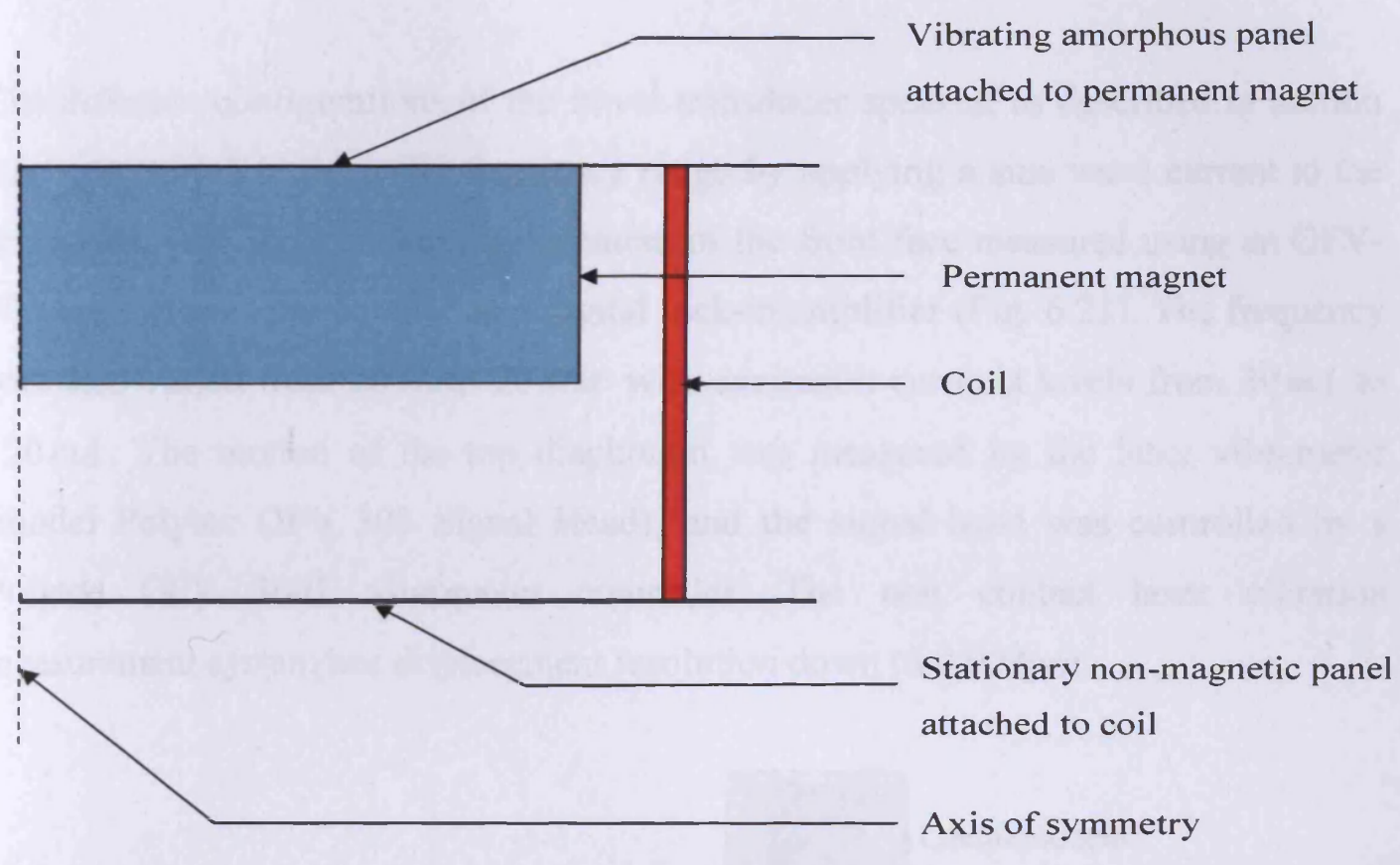

Figure 6.20: An axi-symmetric model of the transducer speaker showing the moving magnet with a non-magnetic bottom layer configuration of the device

Matrix array speakers developed using these transducers have also been tested for understanding the acoustic behaviour, magnetic interactions and the advantages of a multi actuator approach. Intially a $2 \times 2$ matrrix array transducer speaker was constructed using four minature transducers in a matrix configuration. At a later stage a $9 \times 2$ matrrix array transducer speaker using eighteen individual miniature transducers was also developed for experimentation.

Finite element modelling and experimental results explored various device configurations, frequency responses, resonance and the nature and effects of magnetic interactions between transducers. 


\subsection{Experimental Set-Up}

The different configurations of the novel transducer speaker, as described in section 6.6 , were tested in the audio frequency range by applying a sine wave current to the drive coil, with the resultant displacement of the front face measured using an OFV303 laser vibrometer coupled to a digital lock-in amplifier (Fig. 6.21). The frequency was then varied from $20 \mathrm{~Hz}$ to $20 \mathrm{kHz}$ with excitation currents levels from $30 \mathrm{~mA}$ to $120 \mathrm{~mA}$. The motion of the top diaphragm was measured by the laser vibrometer (model Polytec OFV 303 Signal Head), and the signal head was controlled by a Polytec OFV 3001 vibrometer controller. The non contact laser vibration measurement system has displacement resolution down to $0.002 \mu \mathrm{m}$.

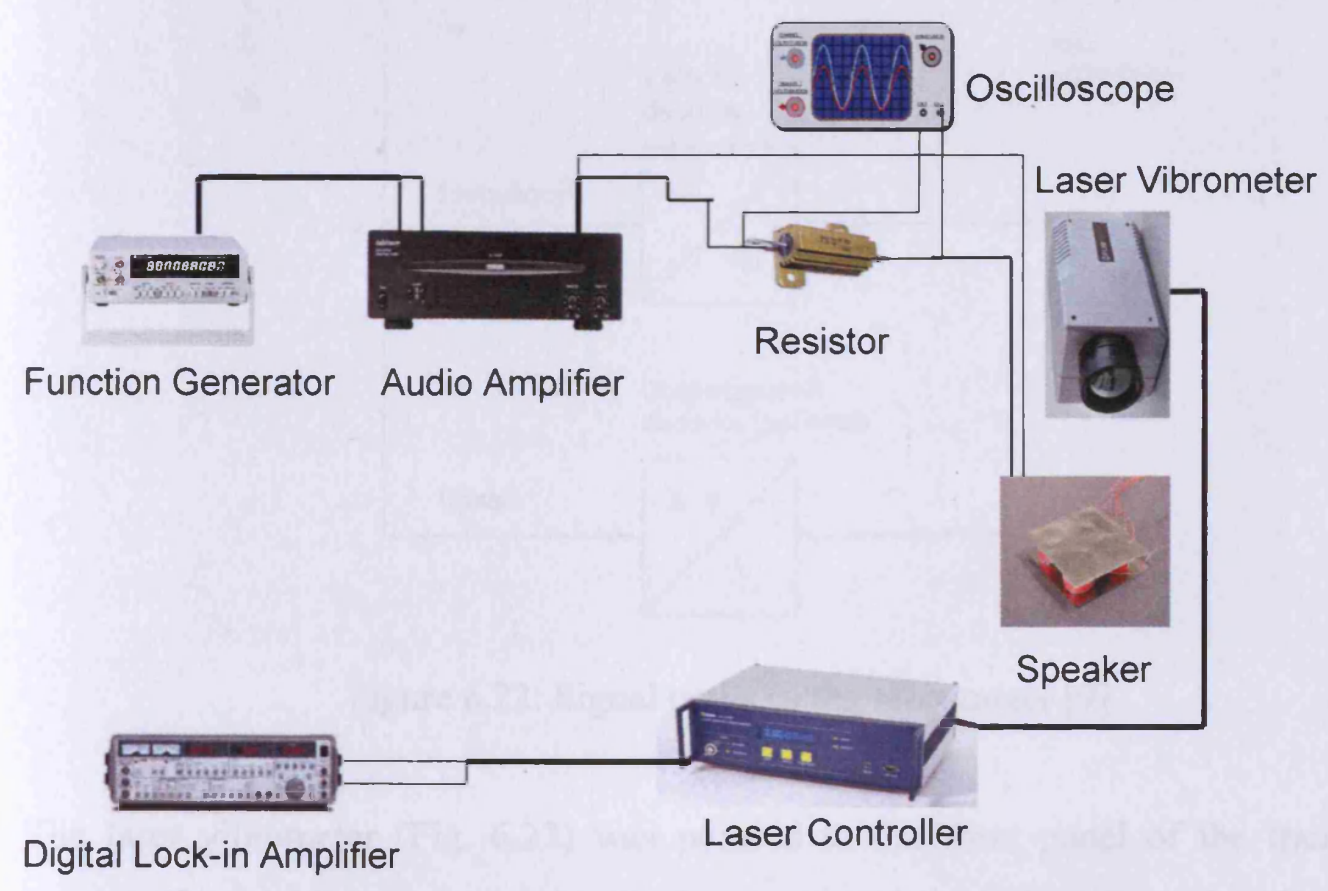

Figure 6.21: Experimental set-up for measuring loudspeaker displacement

Laser vibrometers are instruments for non-contact measurement of surface vibrations based on laser inferometry. The beam of a helium neon laser from the laser vibrometer is focused on the object under investigation, scattered from there and coupled back into the interferometer in the sensor head. The interferometer compares the phase $\varphi_{\bmod }$ and frequency $f_{\bmod }$ of the object beam with those of the internal reference beam $\varphi_{0}$ and $f_{0}$. The frequency difference is proportional to the 
instantaneous velocity and the phase difference is proportional to the instantaneous position of the object. In the controller, the resulting signal is decoded using the velocity decoder and optionally the displacement decoder. Two voltage signals are generated which are respectively proportional to the instantaneous velocity and to the instantaneous position (displacement) of the object [9]. The signal paths in the vibrometer are shown schematically in figure 6.22 [9].

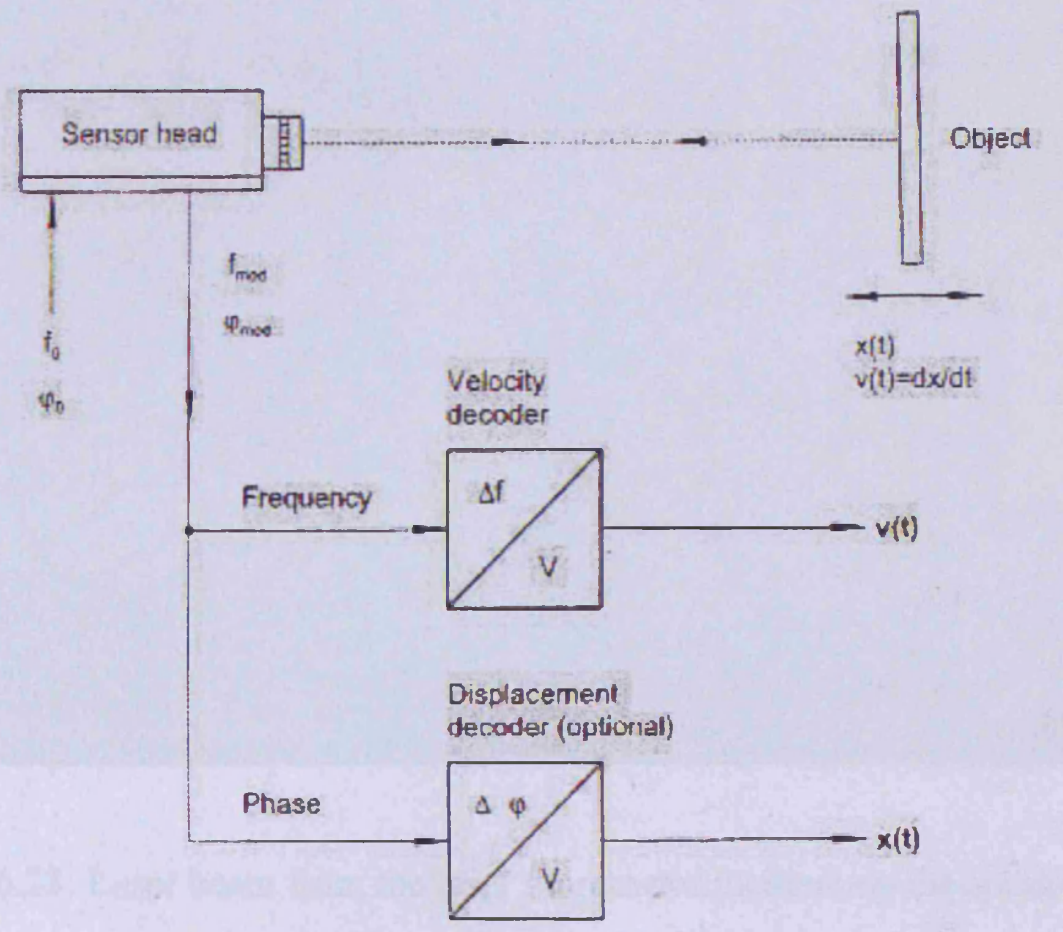

Figure 6.22: Signal paths in the vibrometer [9]

The laser vibrometer (Fig. 6.23) was pointed to the front panel of the transducer speaker (Fig. 6.24) to measure the displacement and the output from the vibrometer was connected to a digital lock-in amplifier. A function generator was used to supply specific voltage and frequency to the actuator through a power amplifier. A $0.1 \mathrm{ohm}$ resistor was connected in series with the amplifier and an oscilloscope was connected across the resistor to measure the current supplied. The instruments used for the measurements of loudspeaker displacement are shown in figure 6.25. All the experiments were carried out in an acoustic anechoic chamber. The experimental results were marginally affected by the inherent low frequency component present in the vibration system and the measuring environment. 


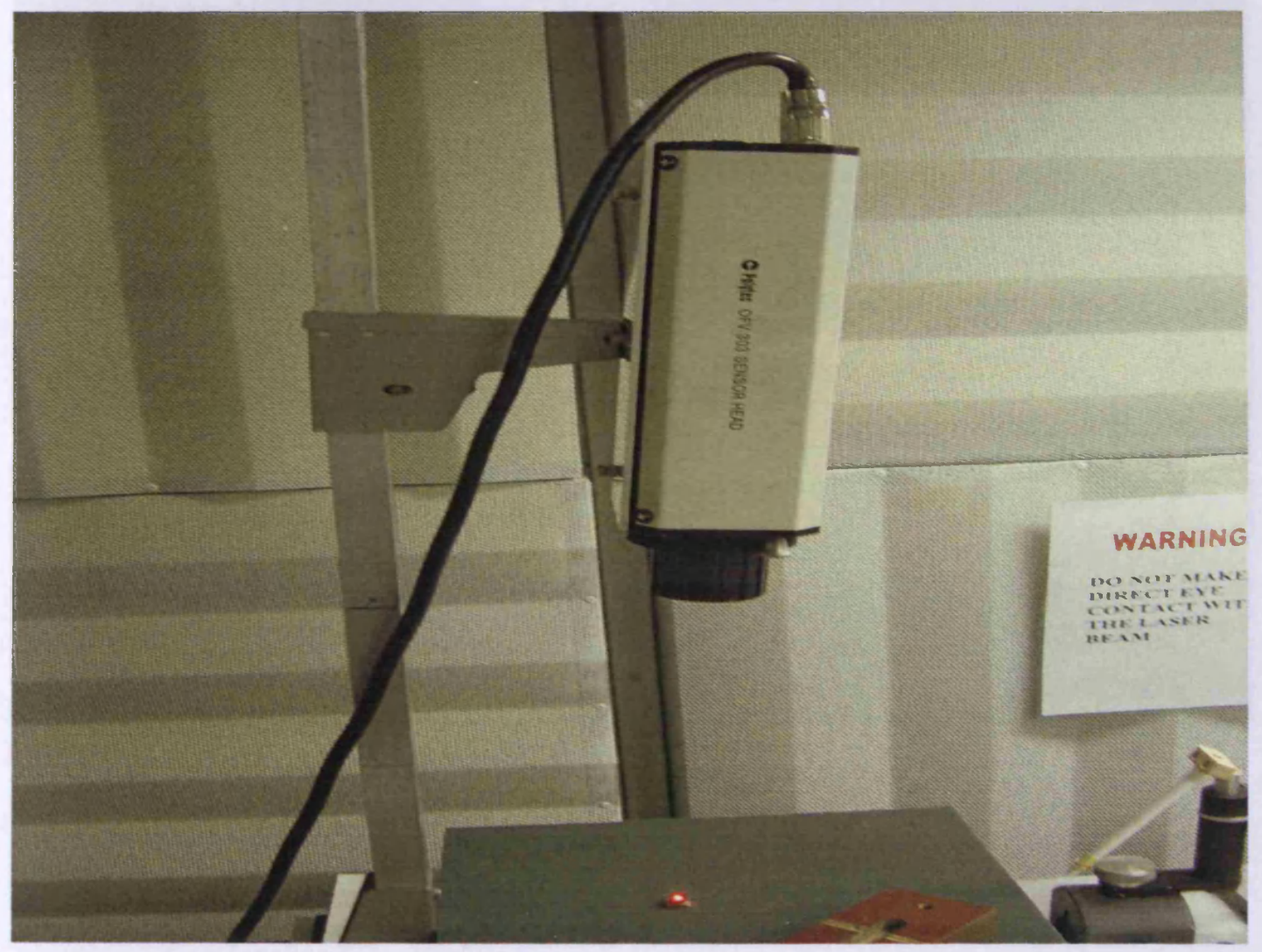

Figure 6.23: Laser beam from the laser vibrometer incident on the speaker panel

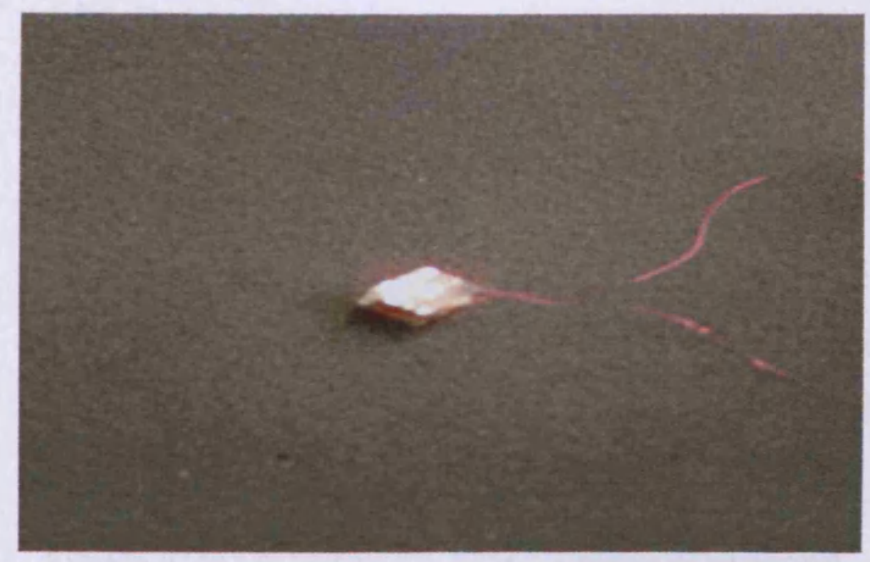

Figure 6.24: A single transducer speaker under test 


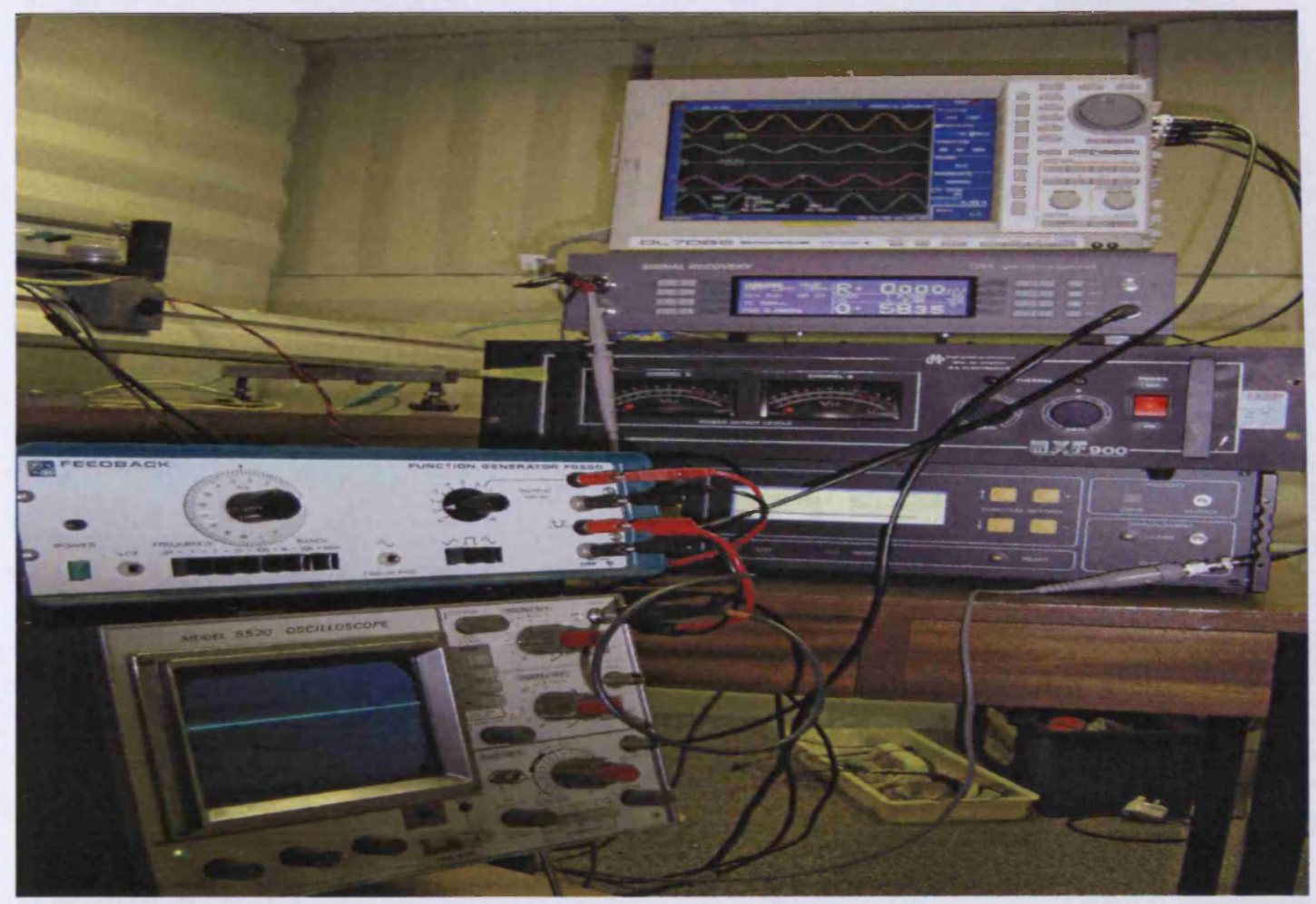

Figure 6.25: Measurement instruments connected for experimentation

In order to measure the sound pressure level from the transducer array speakers, the output from a high bandwidth microphone and an amplifier was connected to a computer with Labview software (Fig. 6.26) using an A/D card. Using the Labview software, the captured sound waves were then processed and converted to the corresponding sound pressure levels for the transducer speakers.

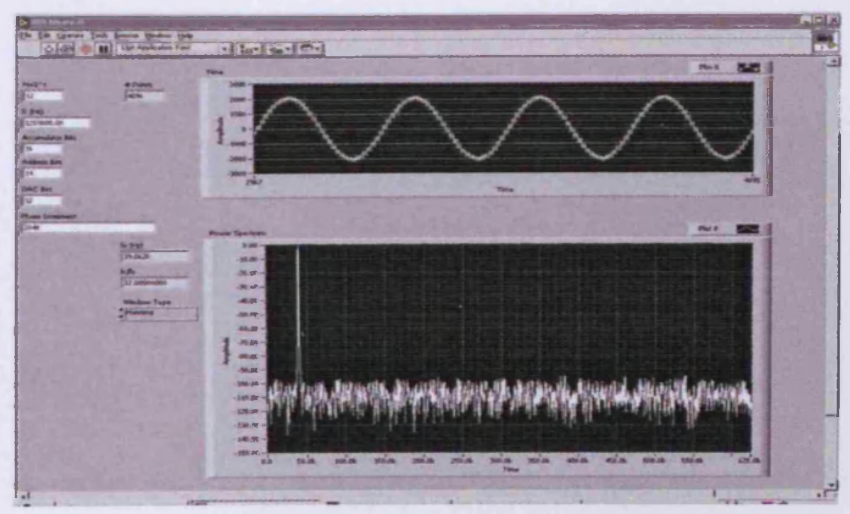

Figure 6.26: A snapshot of the Labview program 
In the measurement of the harmonic distortions of the different configurations, the data from displacement measurements were processed by the SIMPLORER [10] software (Fig. 6.27). The software allowed the identification of the different harmonic contents at audio frequencies.

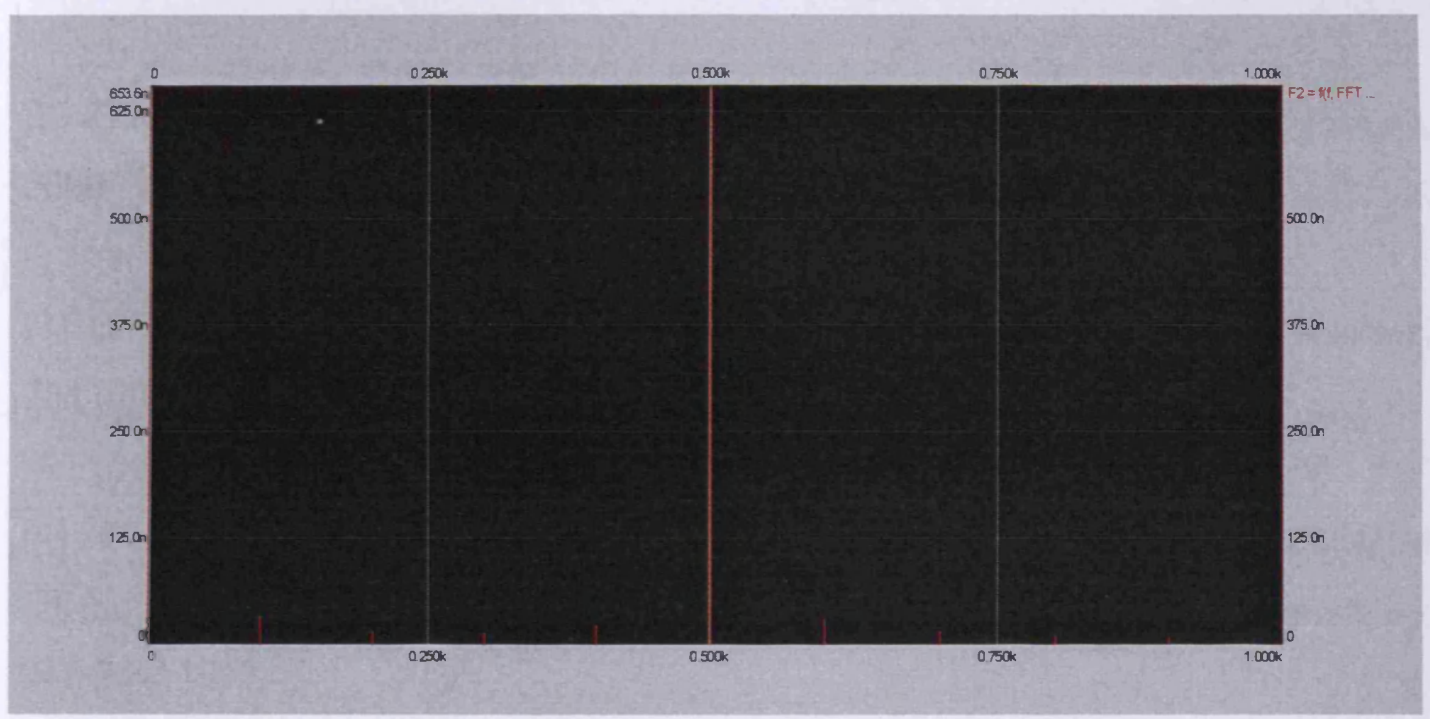

Figure 6.27: A snapshot of the 'SIMPLORER' software measuring harmonic distortions 


\section{References:}

[1] O. Cugat, J. Delamare, G. Reyne, "Magnetic Micro-Actuators and Systems (MAGMAS)," IEEE Trans. on Magnetics, Vol. 39, pp. 3607 - 3612 (2003)

[2] J. R. Brauer, "Magnetic Actuators and Sensors," Wiley-IEEE Press, $1^{\text {st }}$ Edition, 2006

[3] D. Jiles, "Introduction to Magnetism and Magnetic Materials," CRC Press Inc, 2nd Edition, 1998

[4] T. Roschke, G. Gerlach, "An Equivalent Network Model of a Controlled Solenoid", Proc. of the IASTED Intl. Conf. on Applied Modelling and Simulation, pp. 241-244 (1997)

[5] 'OPERA', Electromagnetic Design Software, Vector Fields Limited, 24 Bankside, Kidlington, Oxford, OX5 1JE, UK

[6] J. M Eargle, "Loudspeaker handbook," Kluwer Academic Publishers, $2^{\text {nd }}$ Edition, 2003

[7] Metglas Web Link: http://www.metglas.com/products/page5_1_2.htm

[8] 'MAGNET', Electromagnetic Design Software, Infolytica Limited, C2, Culham Science Centre, Abingdon, Oxon, OX114 3DB, United Kingdom.

[9] Polytec Laser Doppler Vibrometer User Manual

[10] 'SIMPLORER', Electromechanical System Simulation Software, Ansoft Corporate Headquarters, 225 West Station Square Drive, Suite 200, Pittsburgh, PA 15219, USA 


\subsection{Introduction}

The novel electromagnetic miniature transducer speakers were investigated in terms of panel vibration and acoustic radiation. The novel loudspeaker primarily consists of a panel and an exciter. Contrary to conventional flat panel speakers, pistonic motion is encouraged such that the panel vibrates as wholly as possible. Displacement measurement tools have been used to facilitate system integration of the new panel speakers. In particular, laser Doppler vibrometer, finite element analysis, and fast Fourier transform (FFT) are employed to predict panel vibration and the acoustic radiation. Design procedures are also summarized. In order to understand the behaviour of the novel panel speakers, experimental investigations were undertaken to evaluate frequency response of the different configurations, linearity of the current versus displacement relationship in the speakers, resonance, impedance, and harmonic distortion of the miniature transducer speakers. The results revealed the advantages and the limitations of the various configurations of the new flat panel speakers. The most suitable transducer configuration for the matrix array speaker has been investigated. Experiments have also been carried out to observe whether any significant improvement could be achieved by using a bigger matrix array speaker.

The first set of results showed the frequency responses for the moving magnet configuration of the transducer speaker. The moving magnet configuration is the one in which the amorphous panel attached to the permanent magnet vibrates and the other panel which is attached to the coil stays stationary. The second set of results was obtained for the moving coil configuration of the transducer speaker. In this device configuration, the amorphous diaphragm attached to the coil of the speaker vibrates and the other panel that is attached to the magnet remains stationary. The third set of results was obtained for the moving magnet configuration in which the material for the stationary panel, which was originally of amorphous ribbon, was replaced with a non-magnetic one. Further results also showed the characteristics of the moving coil configuration of a single transducer with a non-magnetic diaphragm. 
The variation of harmonic distortions with excitation current and frequency was investigated for the three different device configurations. The other results show the displacement profile and the impedance characteristic of a single transducer speaker. Another set of results showed the effects of neighbouring transducers in a matrix array speaker and explored and compared the frequency responses of two different matrix array speaker systems.

\subsection{Results for Moving Magnet Configuration}

\subsubsection{Displacement versus Frequency Responses}

The moving magnet configuration of the transducer speaker has been tested for frequency responses. Figure 7.1 and 7.2 show the displacement versus frequency responses of the moving magnet transducer configuration in the audio frequency range. In order to obtain the displacement characteristics at various frequencies, the vibration of the diaphragm which was attached to the permanent magnet was measured using a laser Doppler vibrometer. The laser from the vibrometer was incident on the front vibrating panel of the transducer speaker while the frequency was varied from $20 \mathrm{~Hz}$ to $20 \mathrm{kHz}$. At each observed frequency, the excitation current was varied from $30 \mathrm{~mA}$ to $120 \mathrm{~mA}$. 


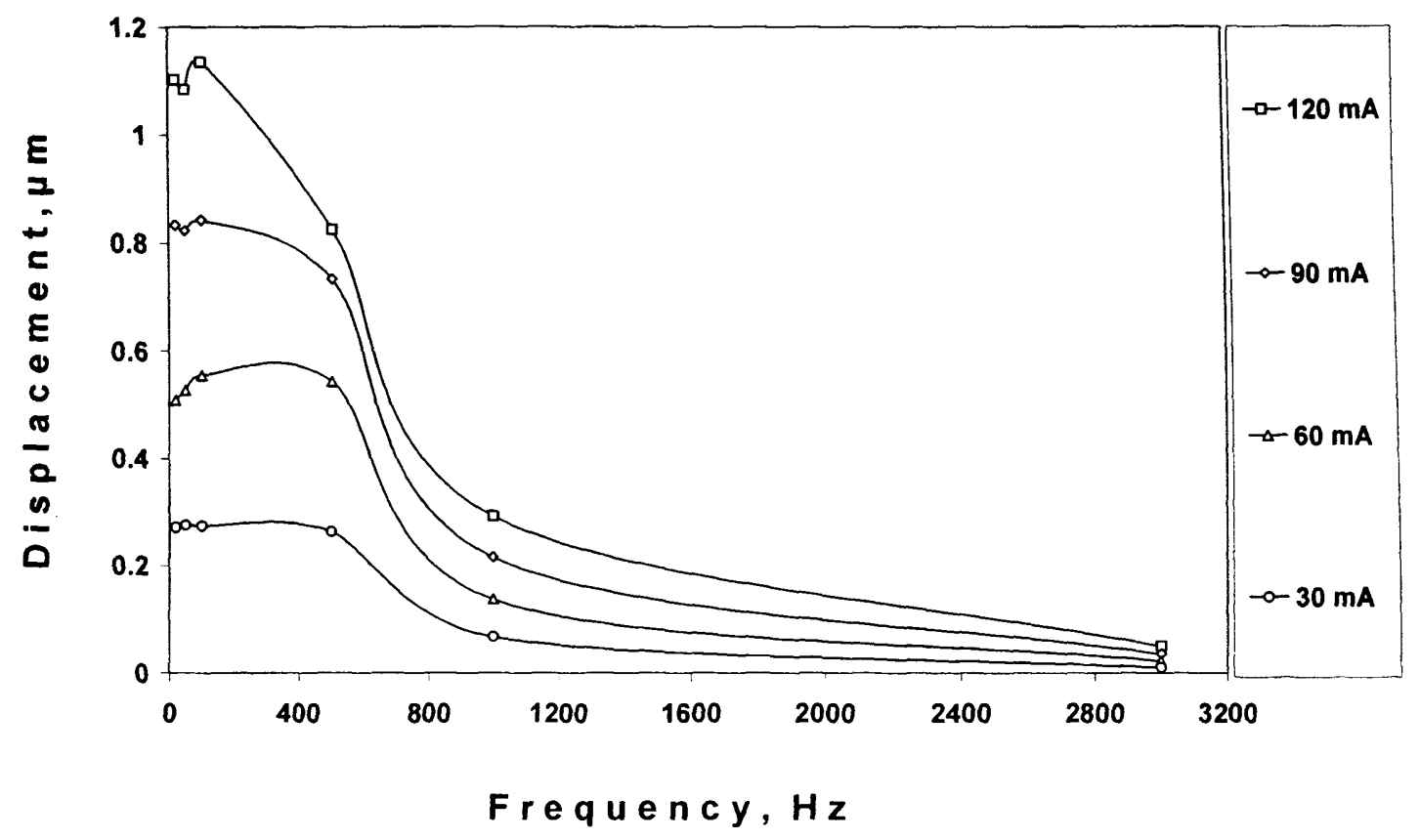

Figure 7.1: Displacement profile (non-resonant) of the moving magnet configuration of the transducer speaker showing displacements in the frequency range of $20 \mathrm{~Hz}$ to 3 $\mathrm{kHz}$ with excitation currents varying from $30 \mathrm{~mA}$ to $120 \mathrm{~mA}$. Displacements measured with laser incident on the centre of the vibrating diaphragm.

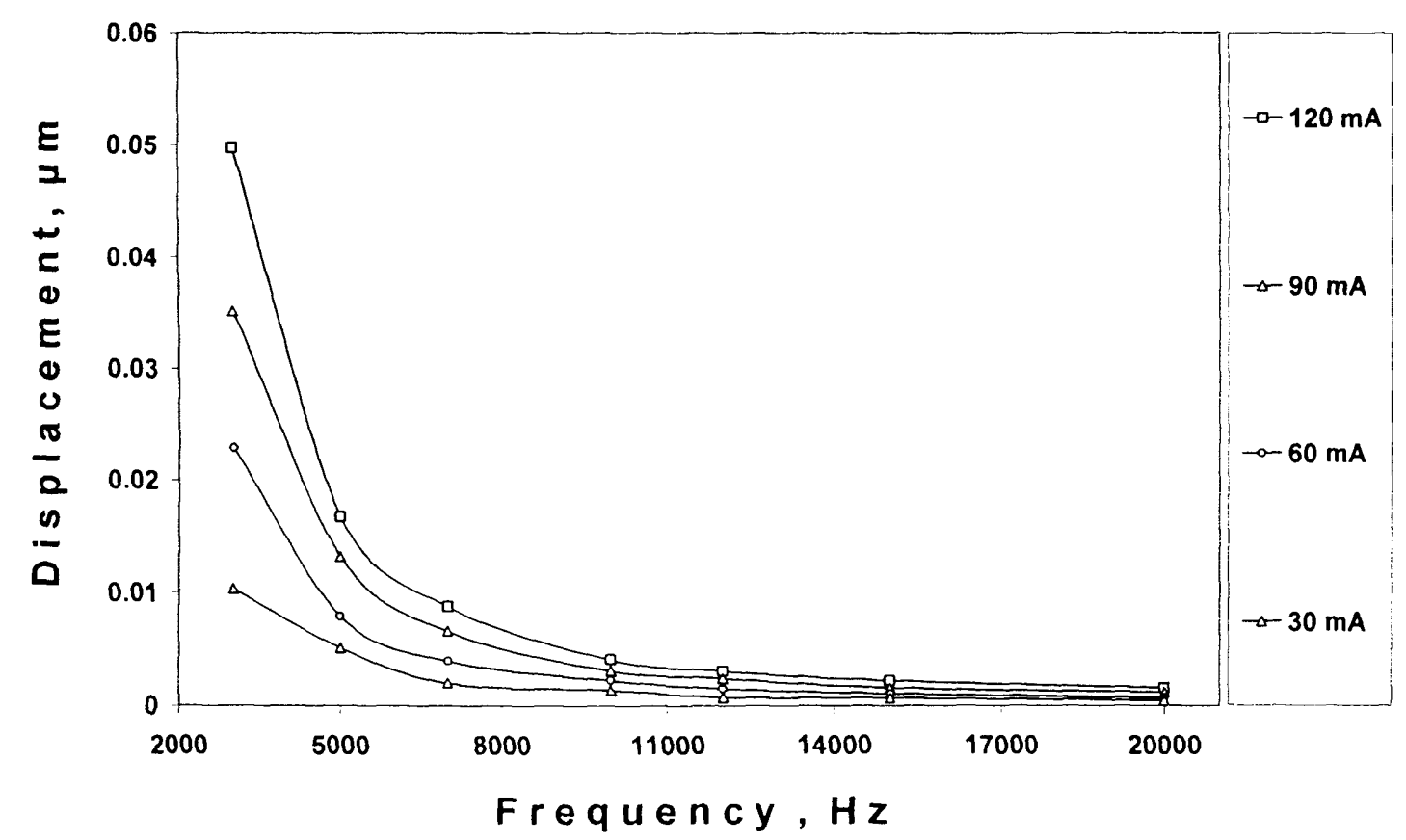

Figure 7.2: Displacement profile (non-resonant) of the moving magnet configuration of the transducer speaker showing displacements in the frequency range of $3 \mathrm{kHz}$ to $20 \mathrm{kHz}$ with excitation currents varying from $30 \mathrm{~mA}$ to $120 \mathrm{~mA}$. Displacements measured with laser incident on the centre of the vibrating diaphragm. 
The low frequency response (Fig. 7.1) for the moving magnet configuration showed a gradual decrease in displacement as frequency increased and an increase in displacements with the increase of excitation current levels. The reduction in displacement with the rise in frequency is also evident in figure 7.2 which showed the high frequency response of the transducer. This feature of the loudspeaker identifies the novel transducer loudspeaker as a 'mass controlled' device. In both cases, in high and low frequency region, the behaviour of the transducer speaker can be explained by examining the relationship between force, mass, acceleration and the velocity of the loudspeaker diaphragm.

The current-magnet combination in the transducer speaker provided a force that is constant with frequency. The diaphragm of the speaker, on the other hand, resisted the force with a mass which is a combination of its own mass and the mass of air. Therefore according to Newton's second law of motion which is:

$$
F=m a
$$

where $F$ is the force, $m$ is the mass and $a$ is the acceleration, it can be deduced from the above equation that the loudspeaker diaphragm obtained constant acceleration with frequency. Now for a simple harmonic motion, the following equations define the displacement, velocity and acceleration:

$$
\begin{aligned}
& \text { Displacement, } x(t)=A \sin (2 \pi f t+\theta) \\
& \text { Velocity, } v(t)=\frac{d x(t)}{d t}=A \cdot(2 \pi f) \cdot \cos (2 \pi f t+\theta)
\end{aligned}
$$

Acceleration, $a(t)=\frac{d v(t)}{d t}=-A \cdot(2 \pi f)^{2} \cdot \sin (2 \pi f t+\theta)=-(2 \pi f)^{2} \cdot x(t)$

In the above equation $A$ is the amplitude, $f$ is the frequency and $\theta$ is the phase angle of the harmonic wave. As it has been concluded from equation 7.1, the diaphragm undergoes constant acceleration and therefore according to the equation 7.4 the magnitude of the displacement is inversely proportional to the square of the signal frequency:

$$
x(t) \propto \frac{1}{f^{2}}
$$


This relationship between the displacement and the frequency in equation 7.5 explains the reduction of displacement with frequency in a loudspeaker.

\subsubsection{Displacement versus Current Responses}

The linearity of the displacements with excitation currents for the moving magnet configuration has been observed in figure 7.3, 7.4 and 7.5. The following figures explore whether the novel transducer can operate as a linear device. This feature is particularly important since, in order to obtain a smooth frequency response, the diaphragm of a speaker needs to move with amplitudes proportional to the driving current. Therefore, for an ideal loudspeaker, a linear current versus displacement relationship is expected.

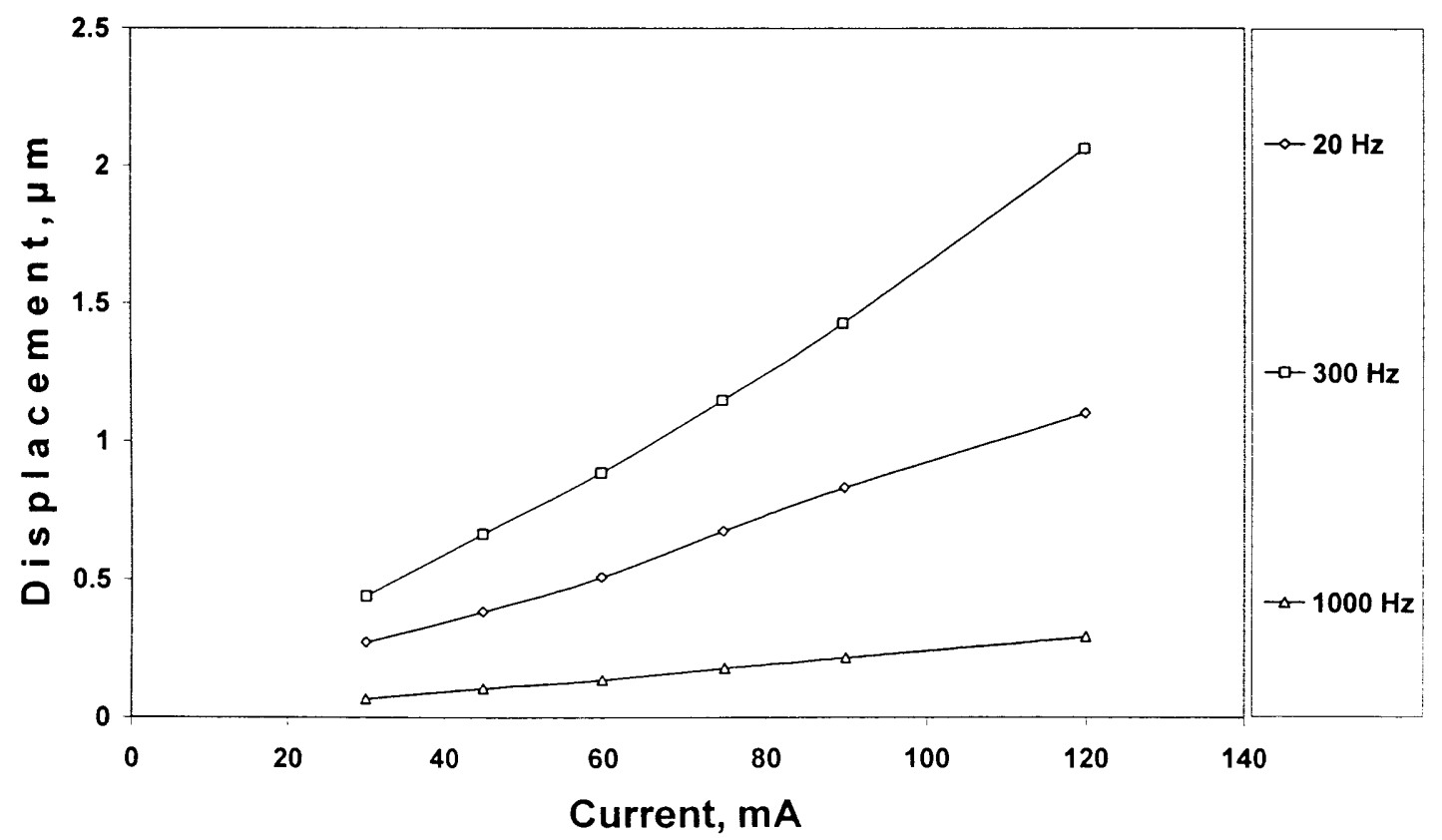

Figure 7.3: Displacement versus current curves for the moving magnet configuration of the transducer speaker with excitation currents varying from $30 \mathrm{~mA}$ to $120 \mathrm{~mA}$ at frequencies $20 \mathrm{~Hz}, 300 \mathrm{~Hz}$ and $1 \mathrm{kHz}$ 


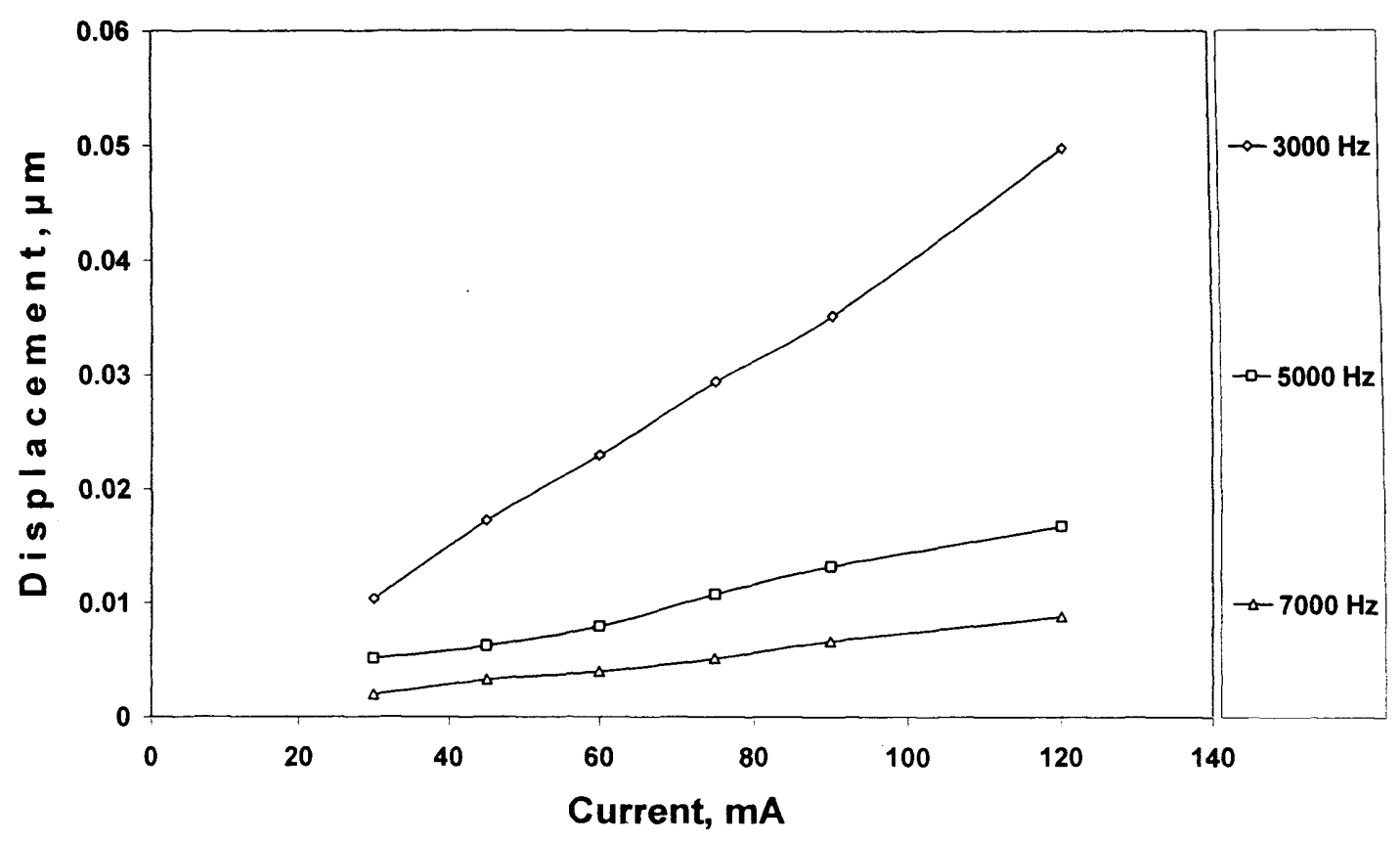

Figure 7.4: Displacement versus current curves for the moving magnet configuration of the transducer speaker with excitation currents varying from $30 \mathrm{~mA}$ to $120 \mathrm{~mA}$ at frequencies $3 \mathrm{kHz}, 5 \mathrm{kHz}$ and $7 \mathrm{kHz}$

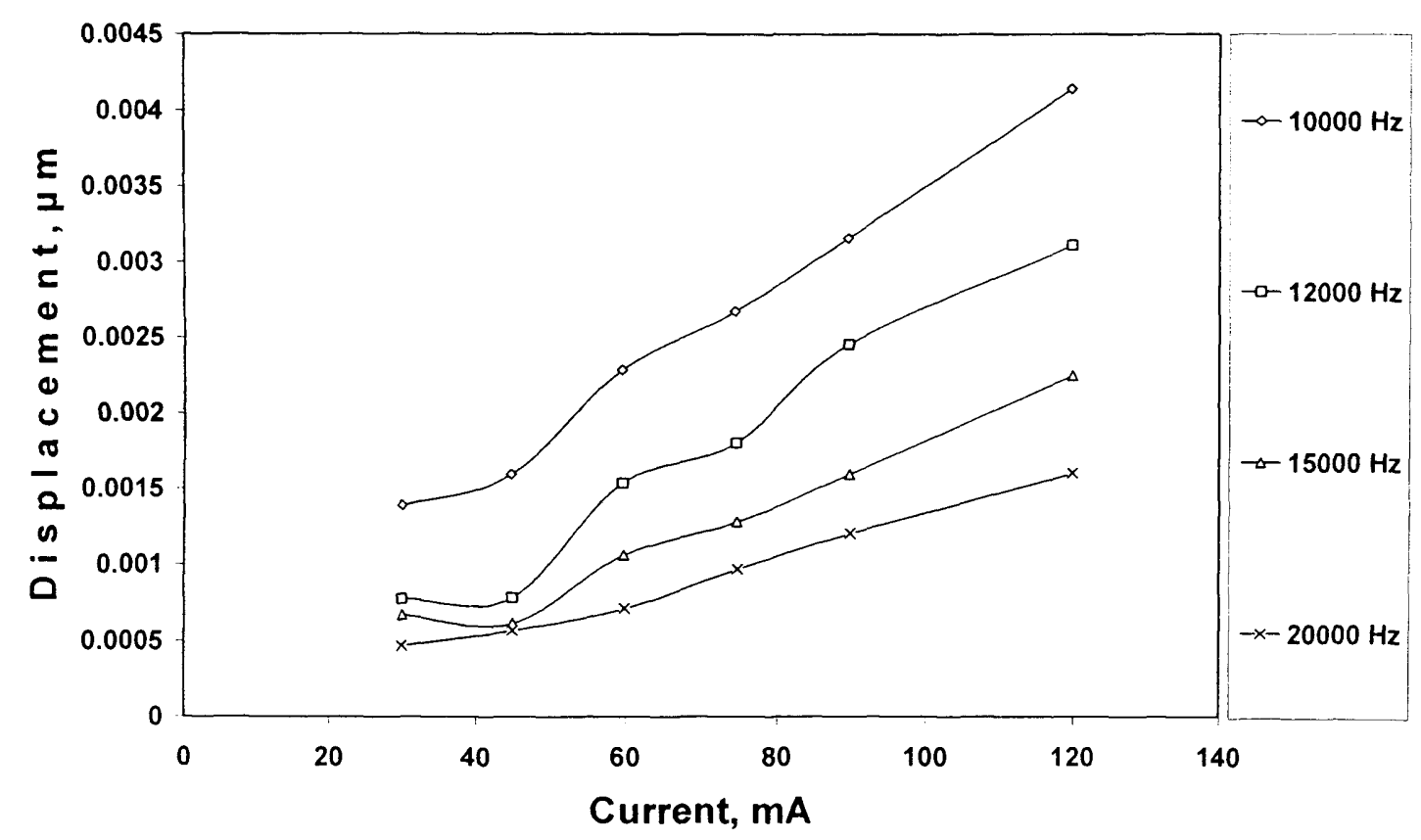

Figure 7.5: Displacement versus current curves for the moving magnet configuration of the transducer speaker with excitation currents varying from $30 \mathrm{~mA}$ to $120 \mathrm{~mA}$ at frequencies $10 \mathrm{kHz}, 12 \mathrm{kHz}, 15 \mathrm{kHz}$ and $20 \mathrm{kHz}$ 
This set of graphs explored the displacement-current relationship in the novel transducer speaker. For a typical loudspeaker application, an ideal displacement versus current curve would be linear. Now in the case of novel transducer speaker three different curves (Figs. 7.3, 7.4 and 7.5) showed the displacement-current relationship at low, mid and high range frequencies.

At low frequency region, the displacement versus current relationship is linear at lower excitation currents. However, as the excitation current increased, non-linearity was introduced in the response. As it can be observed in the displacement versus frequency results (Figs. 7.1 and 7.2), the displacement is higher at low frequencies and therefore higher excitation current leads to even higher displacements at low frequencies. When the diaphragm of the transducer speaker approached the maximum excursion point, non-linearity was introduced to the system. In the case of the novel transducer speaker, the maximum excursion is limited by the physical size of the speaker diaphragm. The small size of the miniature speaker is not suitable for producing longer wavelengths; therefore the non-linear response was particularly visible for longer wavelengths with higher amplitudes.

In the high frequency region, on the other hand, non-linearity or distortion is visible in lower excitation currents. This is primarily because at higher frequencies, the diaphragm of the speaker moves much faster and since this was a moving magnet configuration, the added weight of the permanent magnet made it difficult to obtain smooth excursions at lower excitation currents. The lack of enough driving force in lower excitation currents introduced non-linearity in the response of the transducer speaker at high frequencies. 


\subsubsection{Acoustic Intensity versus Frequency Response}

Figure 7.6 shows the acoustic intensity versus frequency response for the moving magnet configuration of the transducer speaker. This figure shows the acoustic power radiated by a vibrating diaphragm at various frequencies in the audible frequency range. The acoustic intensity response also indicates the suitability of a transducer speaker for a given frequency range.

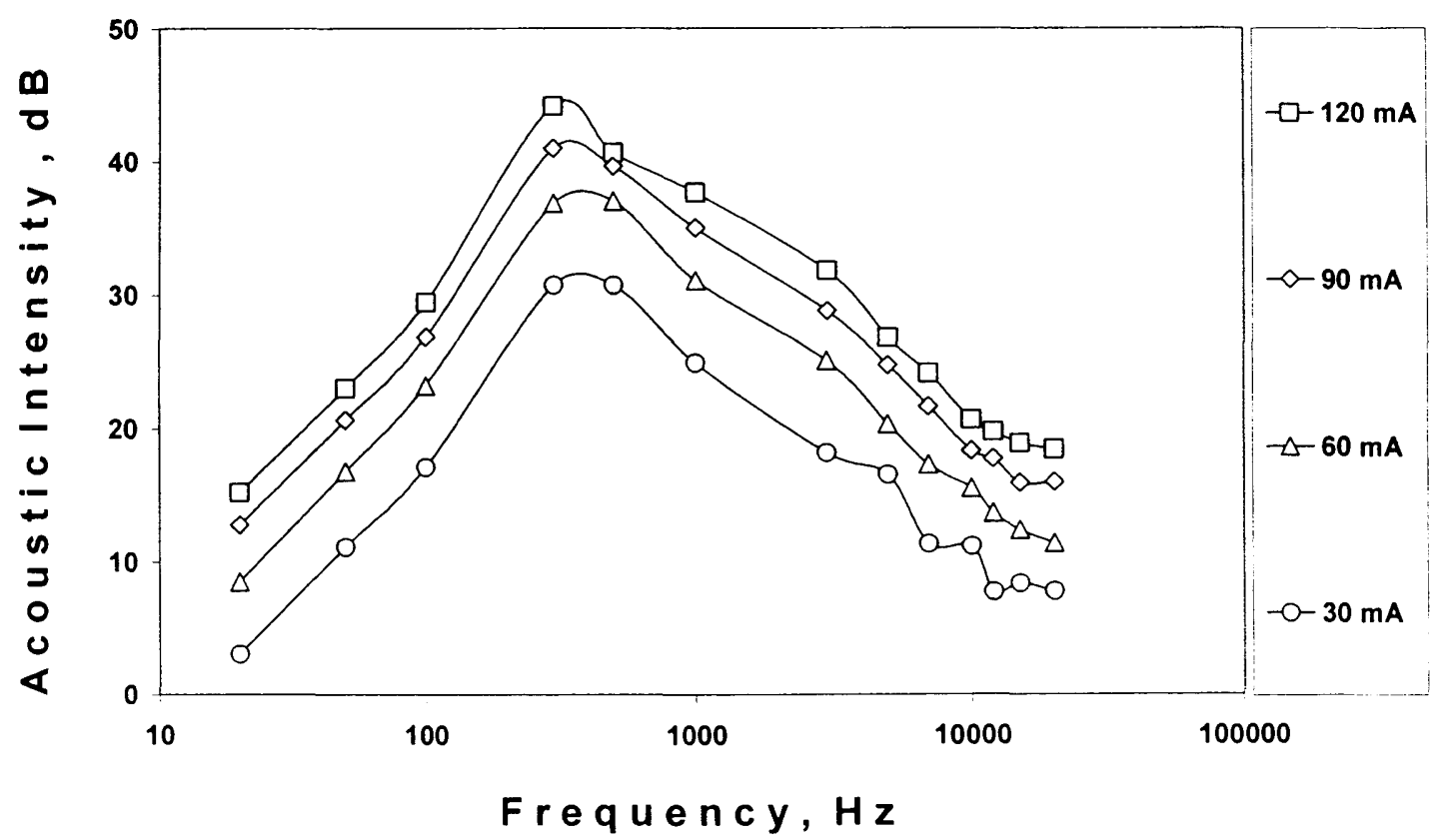

Figure 7.6: Acoustic intensity curves for the moving magnet configuration of the transducer speaker showing acoustic intensity in decibel $(\mathrm{dB})$ for frequencies varying from $20 \mathrm{~Hz}$ to $20 \mathrm{kHz}$ at various excitation currents ranging from $30 \mathrm{~mA}$ to $120 \mathrm{~mA}$

It can be seen from the above graph that, in the low frequency region, the acoustic intensity increased gradually with the increase in frequency. This indicates that the physical size of the sound radiating diaphragm limited the low frequency sound production in the miniature transducer speakers. However as the frequency was increased, the transducer speaker was able to deliver more acoustic output. The low frequency limitation is also due to moving magnet combination of the speaker. The moving load is heavier in the moving magnet combination because of the mass of the 
permanent magnet and hence the added weight limited the higher displacements required at the low end of the audio frequency range. At high frequency region, the acoustic intensity dropped down gradually as the frequency increased. As the frequency of the excitation current increased, the speed at which the loudspeaker diaphragm moves also needs to increase. However, in the case of a moving magnet configuration, the added weight of the permanent magnet makes the moving diaphragm heavier and increasingly difficult to move at higher frequency.

However, instead of having low acoustic intensity the sound was still audible at higher frequencies, whereas at lower frequencies the sound was not audible below 3 $\mathrm{KHz}$ instead of having similar acoustic intensity. This behaviour relates to the perception of loudness by the human ear (Fig. 7.7) [1]. The intensity of the sound and loudness is not the same thing as far as the sensitivity of ear is concerned. The human ear is more sensitive in the high frequency region compared to the low frequency one. Therefore, even if the intensity of the sound is lower or similar in the high frequency region compared to that of the low frequencies, sound was still audible. On the other hand, the low frequency sound was not audible even if the intensity at low frequencies was similar to that of the high frequencies since the intensity required for a low frequency sound to be audible is much higher than that required for a high frequency sound to be audible.

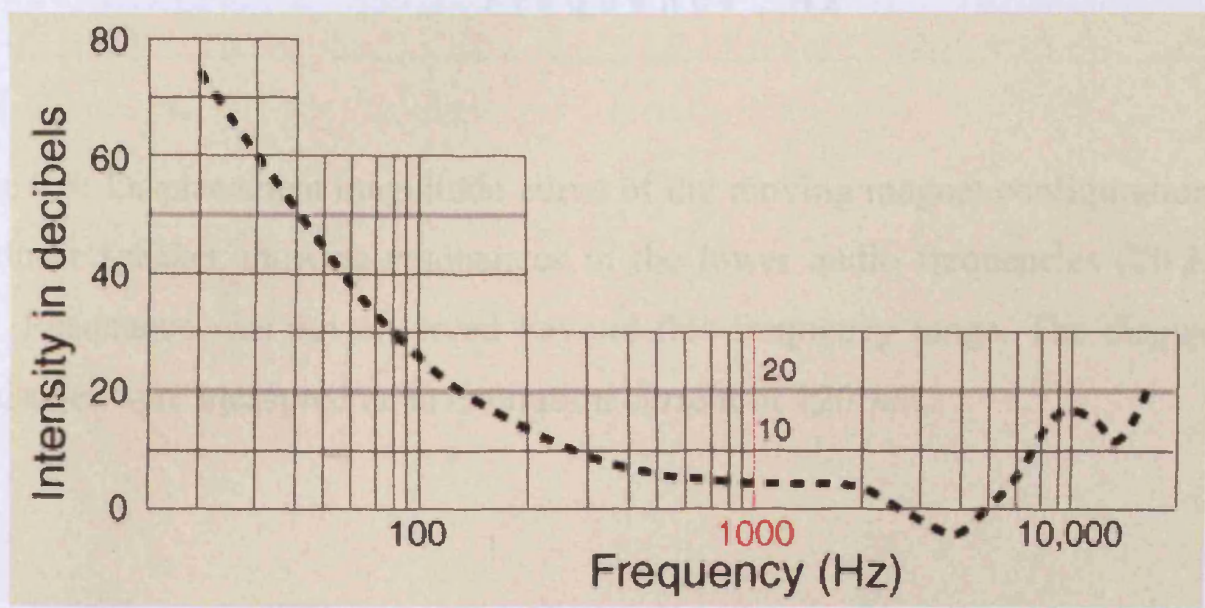

Figure 7.7: Threshold sensitivity curve for the human ear [1] 


\subsubsection{Resonance Curves}

The displacement profile of a transducer speaker along with the phase characteristics over the whole audio frequency range shows the presence of resonance in the transducer's operation. The operation characteristics of a transducer speaker system can be affected by resonances and hence it is important to identify the resonant peaks in the system. The resonant curves (Figs. 7.8 and 7.9) showed the resonant behaviour of the miniature transducer speaker within the audible frequency range. Figures 7.8 and 7.9 showed the displacement versus frequency and phase versus frequency curves respectively.

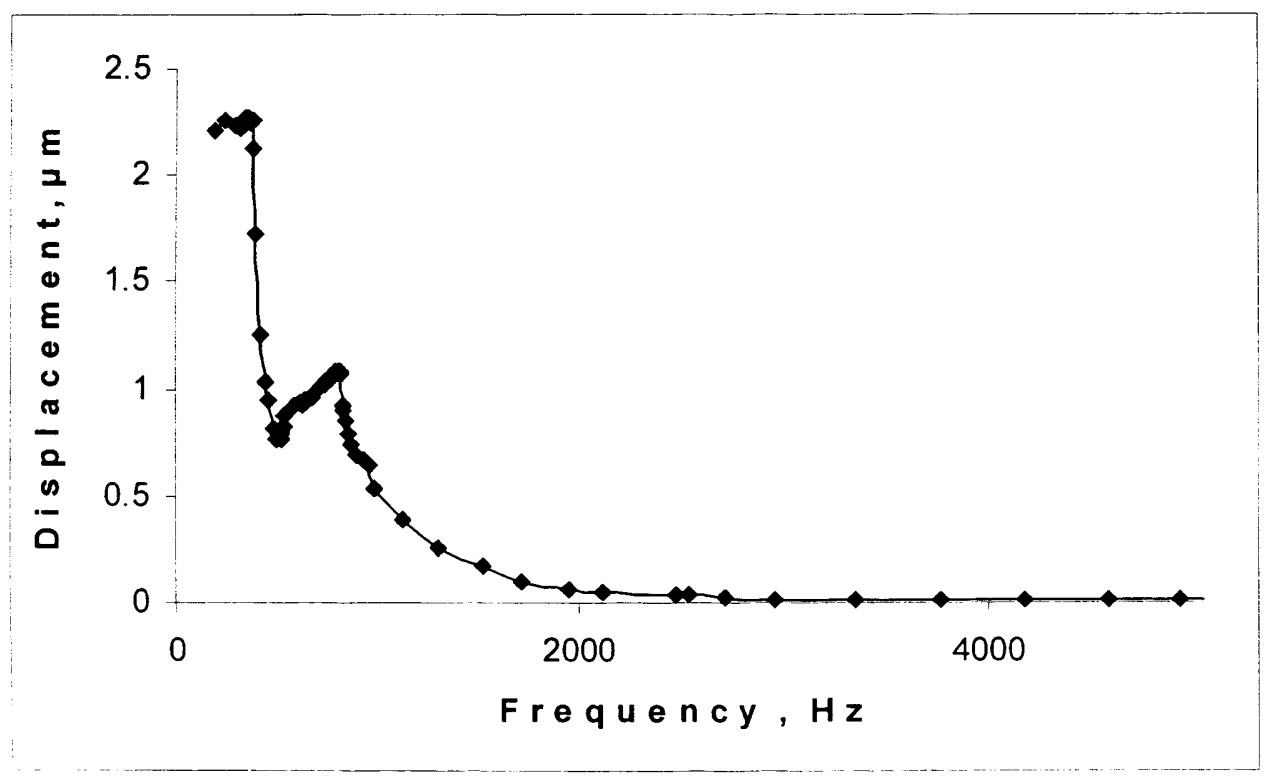

Figure 7.8: Displacement magnitude curve of the moving magnet configuration of the transducer speaker showing resonances in the lower audio frequencies $(20 \mathrm{~Hz}$ to 5 $\mathrm{kHz}$ ). Resonance was not observed beyond this frequency range. The displacement magnitudes were measured at an excitation current of $120 \mathrm{~mA}$. 


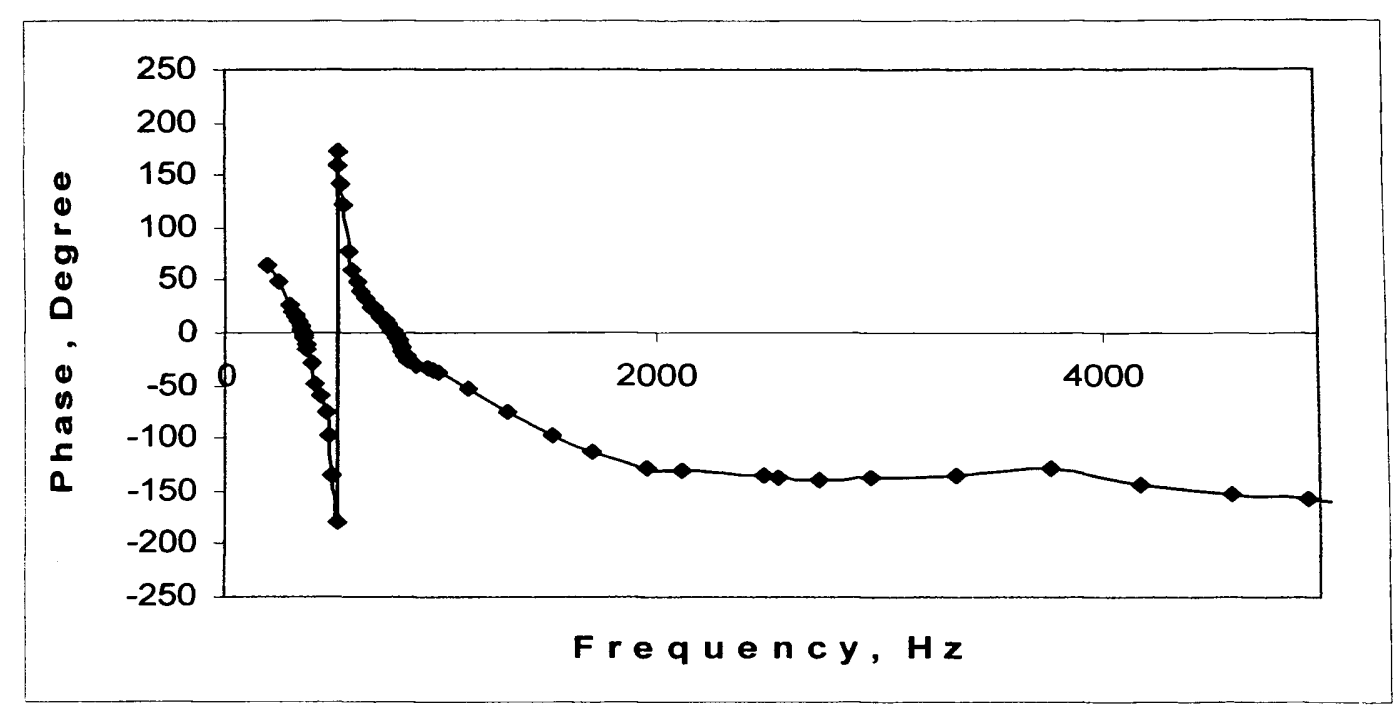

Figure 7.9: Phase curve of the moving magnet configuration of the transducer speaker showing resonances in the lower audio frequencies $(20 \mathrm{~Hz}$ to $5 \mathrm{kHz})$. Resonance was not observed beyond this frequency range. The phase information was recorded at an excitation current of $120 \mathrm{~mA}$.

The magnitude of the displacement and the corresponding phase indicate that there is a resonant peak in the low-frequency region. The resonant peak was observed around $500 \mathrm{~Hz}$ for the moving magnet combination. However, in the high-frequency region $(>2 \mathrm{kHz}$ ) no additional resonances were observed. Therefore, apart from the lowfrequency end of the audio spectrum, the novel transducer speaker could reproduce a linear sound output across the whole audible frequency range. 


\subsubsection{Acoustic Intensity versus Current Response}

The acoustic intensity versus current responses showed the linearity of acoustic intensity with excitation currents at various frequencies. Figure 7.10, 7.11 and 7.12 shows the acoustic intensity versus driving current at low, medium and high frequencies, respectively, for the moving magnet configuration. In order to deliver acoustic power in proportion to the driving current over the whole audio frequency range, a linear acoustic intensity versus current response is expected from an ideal loudspeaker.

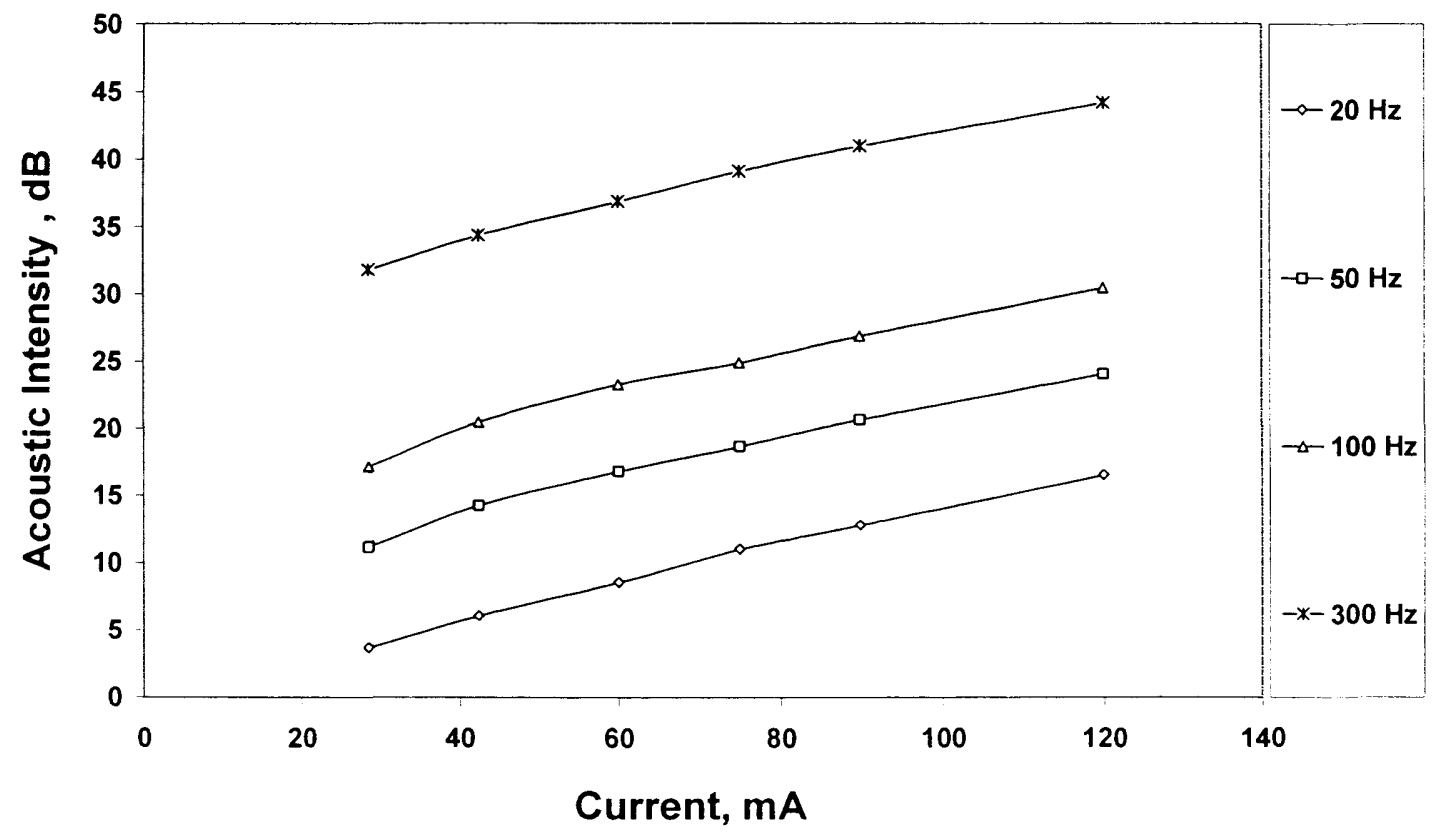

Figure 7.10: Acoustic intensity versus current curves for the moving magnet configuration of the transducer speaker showing acoustic intensities with excitation currents varying from $30 \mathrm{~mA}$ to $120 \mathrm{~mA}$ at frequencies $20 \mathrm{~Hz}, 50 \mathrm{~Hz}, 100 \mathrm{~Hz}$ and $300 \mathrm{~Hz}$ 


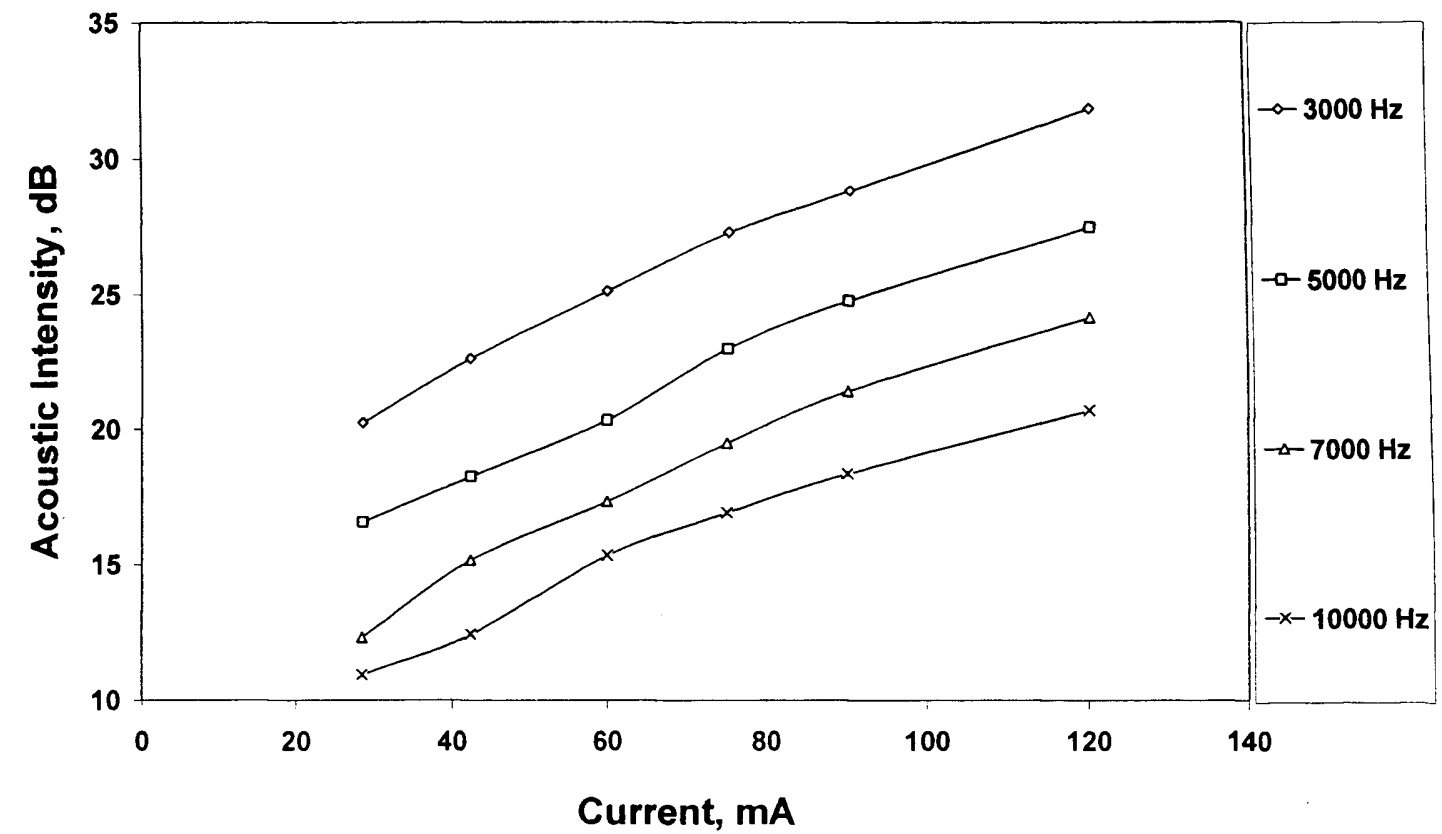

Figure 7.11: Acoustic intensity versus current curves for the moving magnet configuration of the transducer speaker showing acoustic intensities with excitation currents varying from $30 \mathrm{~mA}$ to $120 \mathrm{~mA}$ at frequencies $3 \mathrm{kHz}, 5 \mathrm{kHz}, 7 \mathrm{kHz}$ and 10 $\mathrm{kHz}$

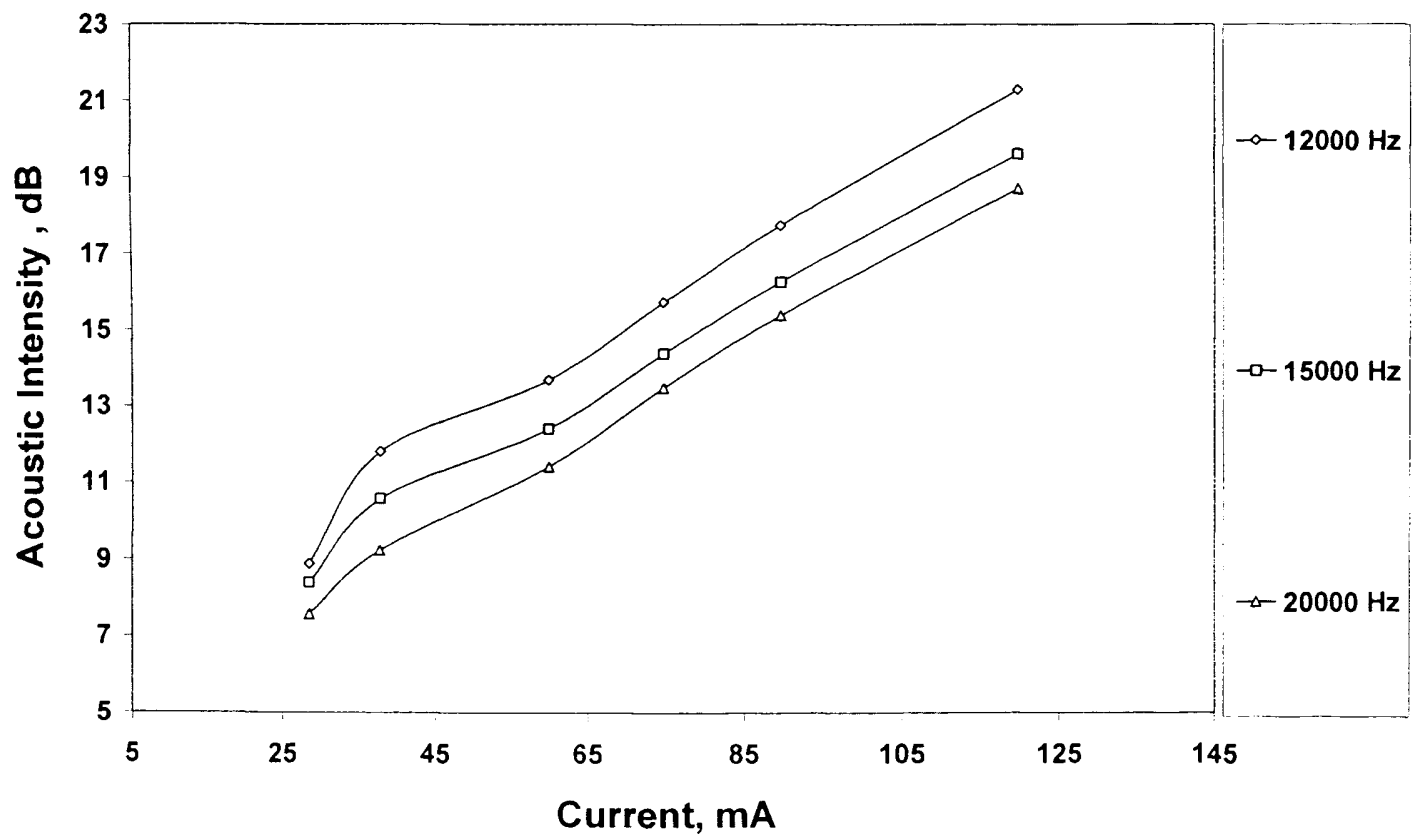

Figure 7.12: Acoustic intensity versus current curves for the moving magnet configuration of the transducer speaker showing acoustic intensities with excitation currents varying from $30 \mathrm{~mA}$ to $120 \mathrm{~mA}$ at frequencies $12 \mathrm{kHz}, 15 \mathrm{kHz}$ and $20 \mathrm{kHz}$ 
The intensity versus current curves at various frequency regions followed a linear trend in which the acoustic intensity of the transducer speaker increased linearly with the increase in the excitation current. Using the linear curve fitting technique, a confidence value of 0.99 was obtained for these curves. Therefore it can be assumed that the excitation current versus acoustic intensity relationship was linear. This characteristic of the novel transducer speaker is important in terms of the control of loudspeaker since it assumes the transducer to be a controlled linear device. This characteristic also ensured that the novel transducer speaker can deliver acoustic power linearly over the whole audible frequency range. However, in the high frequency region (Fig. 7.12), the transducer exhibited distortion in the lower excitation currents. The reason for such distortion can be explained by the same factor that introduced distortion in the displacement response at high frequency and low excitation currents. A moving magnet loudspeaker diaphragm requires extra force to move at high frequency because the high frequency movement of the moving magnet loudspeaker diaphragm is limited at lower excitation current by the weight of the permanent magnet attached to its surface. 


\subsection{Results for Moving Coil Configuration}

\subsubsection{Displacement versus Frequency Responses}

In the moving coil configuration of the novel transducer array speaker, the moving diaphragm was attached to the coil instead of the permanent magnet as described before. Therefore the moving mass of the diaphragm was lighter than in the moving magnet configuration. This reduction in the moving mass of the diaphragm had a significant effect in terms of the frequency response of the loudspeaker. Figure 7.13 and figure 7.14 shows the displacement behaviour of the moving coil configuration over the whole audible frequency range.

In order to obtain the displacement characteristics at various frequencies, the vibration of the diaphragm, which was attached to the coil, was measured using a laser Doppler vibrometer. The laser from the vibrometer was incident on the front vibrating panel of the transducer speaker while the frequency was varied from $20 \mathrm{~Hz}$ to $20 \mathrm{kHz}$. At each observed frequency, the excitation current was varied from $30 \mathrm{~mA}$ to $120 \mathrm{~mA}$.

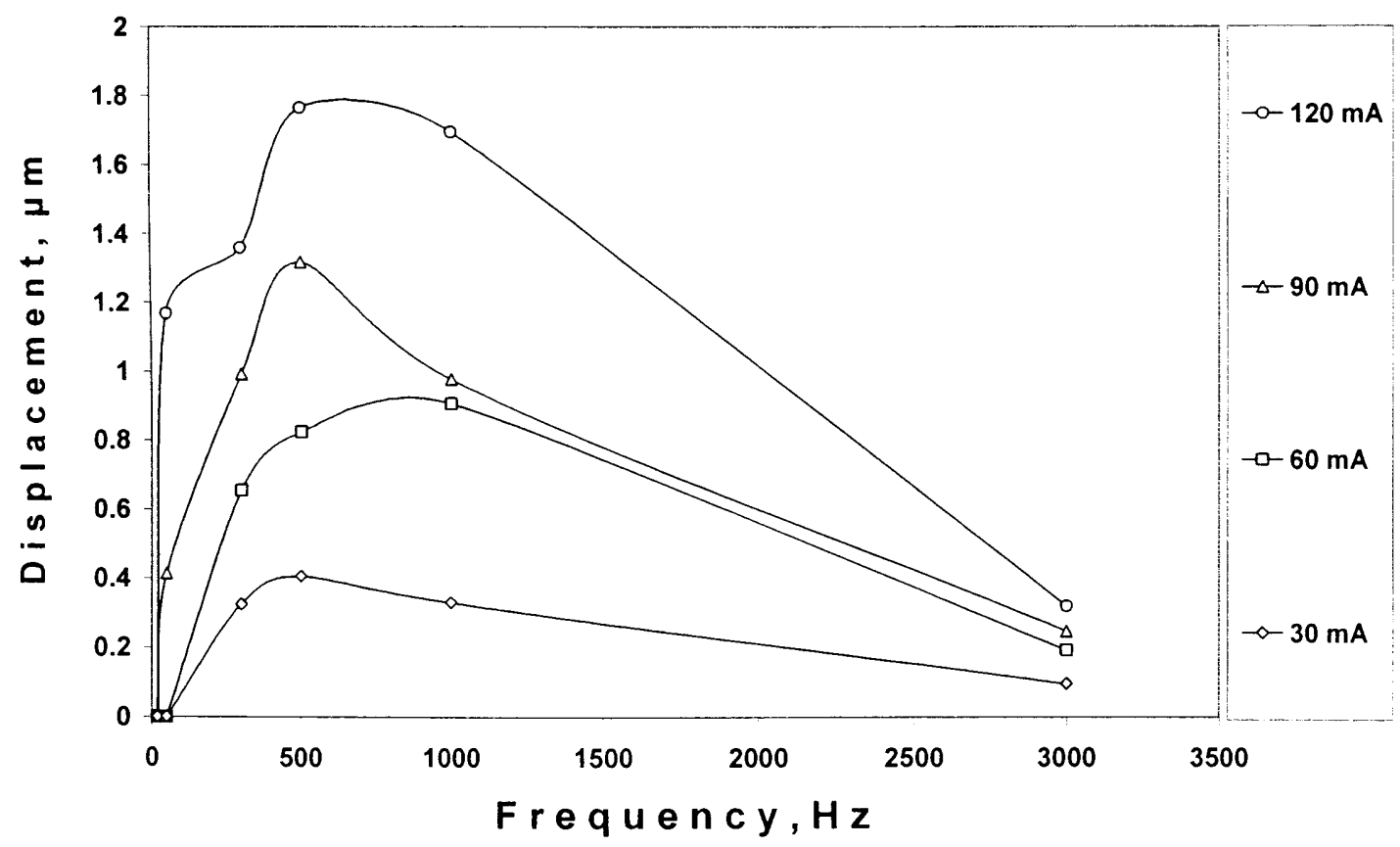

Figure 7.13: Displacement profile (non-resonant) of the moving coil configuration of the transducer speaker showing displacements in the frequency range of $20 \mathrm{~Hz}$ to 3 $\mathrm{kHz}$ with excitation currents varying from $30 \mathrm{~mA}$ to $120 \mathrm{~mA}$. Displacements measured with laser incident on the centre of the vibrating diaphragm. 


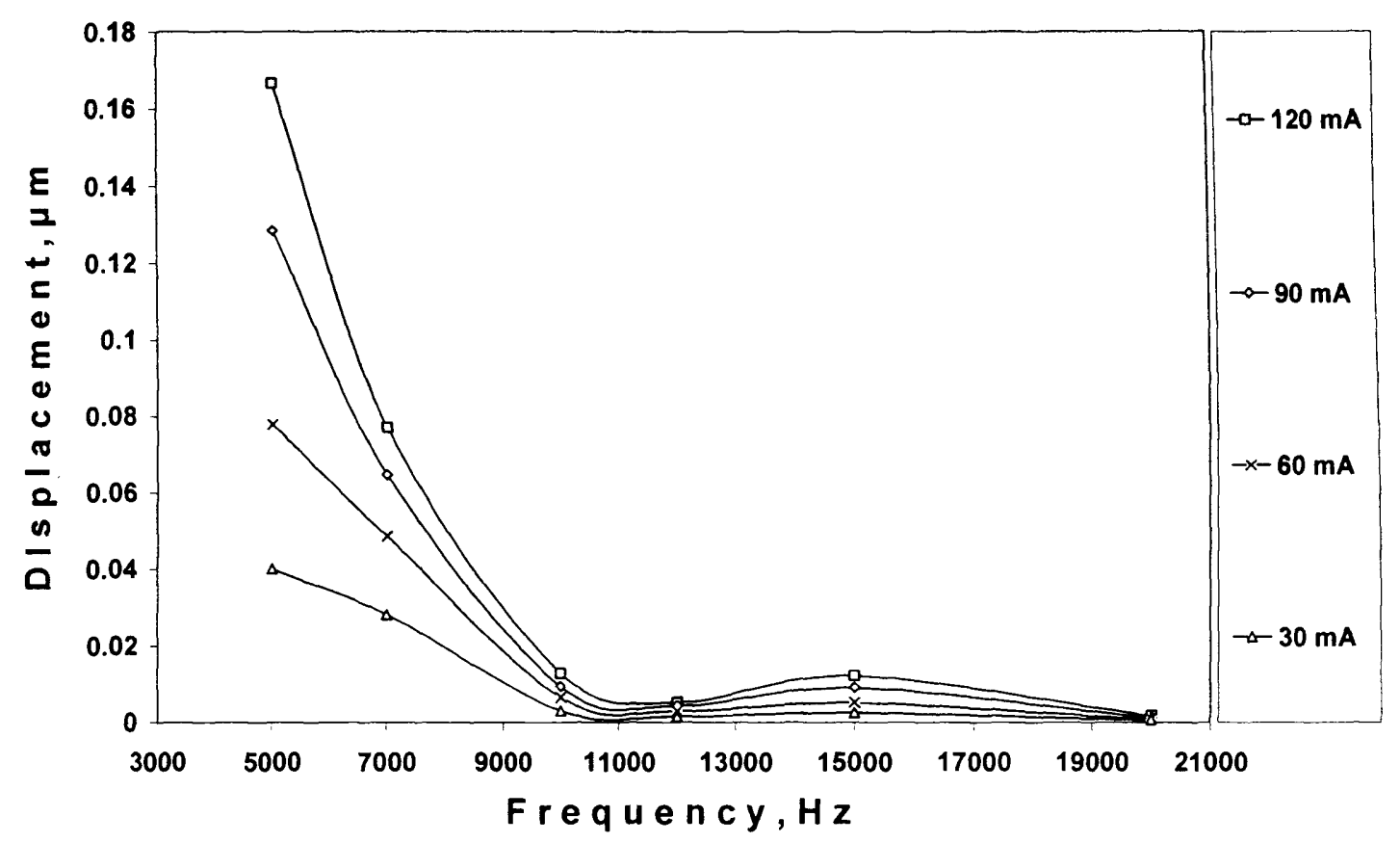

Figure 7.14: Displacement profile (non-resonant) of the moving coil configuration of the transducer speaker showing displacements in the frequency range of $5 \mathrm{kHz}$ to 20 $\mathrm{kHz}$ with excitation currents varying from $30 \mathrm{~mA}$ to $120 \mathrm{~mA}$. Displacements measured with laser incident on the centre of the vibrating diaphragm.

The low frequency displacement behaviour of the moving coil transducer (Fig. 7.13) shows the limitation of the moving coil configuration at the very low end of the audio frequency spectrum at lower excitation currents. Especially below $500 \mathrm{~Hz}$, the diaphragm movement was very low. At low excitation currents (30 $\mathrm{mA}$ and $60 \mathrm{~mA}$ ), the single transducer speaker could not produce any displacement below $300 \mathrm{~Hz}$. However, by employing higher excitation currents, the moving coil transducer speaker was able to produce displacements well below $300 \mathrm{~Hz}$. The reasons for such low frequency limitation at lower excitation currents are the physical size and the lighter weight of the moving diaphragm. The small size of the transducer diaphragm itself served as a limiting factor for producing low frequency sound because a small diaphragm is ideally suited for producing shorter wavelengths at high frequency. In order to produce longer wavelengths, the vibrating diaphragm needs to be larger. The vibrating diaphragm also needs to be heavier in order to match the heavier air mass at 
low frequency. The lighter mass of the moving diaphragm and lower force due to lower excitation currents resulted in the limited low frequency operation. However, as the excitation current was increased, the moving coil transducer speaker was able to produce displacements. The increased force at higher excitation currents compensated the acoustic mismatches and created a balancing vibrating force that could produce displacements at the low end of the audio spectrum.

In the high frequency region (Fig. 7.14), however, the moving coil configuration produced higher displacements than the moving magnet one (Fig. 7.2). The better high frequency response of the moving coil was due to the reduced mass of the moving diaphragm which could vibrate with higher acceleration easily without the added weight of the permanent magnet. This reduced weight of the vibrating diaphragm also resulted in a flatter acoustic response for the moving coil configuration compared to that of a moving magnet configuration.

\subsubsection{Displacement versus Current Responses}

The linearity of the displacements with excitation currents has been observed for the moving coil configuration of the transducer speaker (Figs. 7.15, 7.16 and 7.17). In order to obtain a predictable steady response, the diaphragm of a speaker needs to move with amplitudes proportional to the driving current. The following figures show whether the novel transducer speaker can operate as a linear device in which the output displacement is proportional to the input current. 


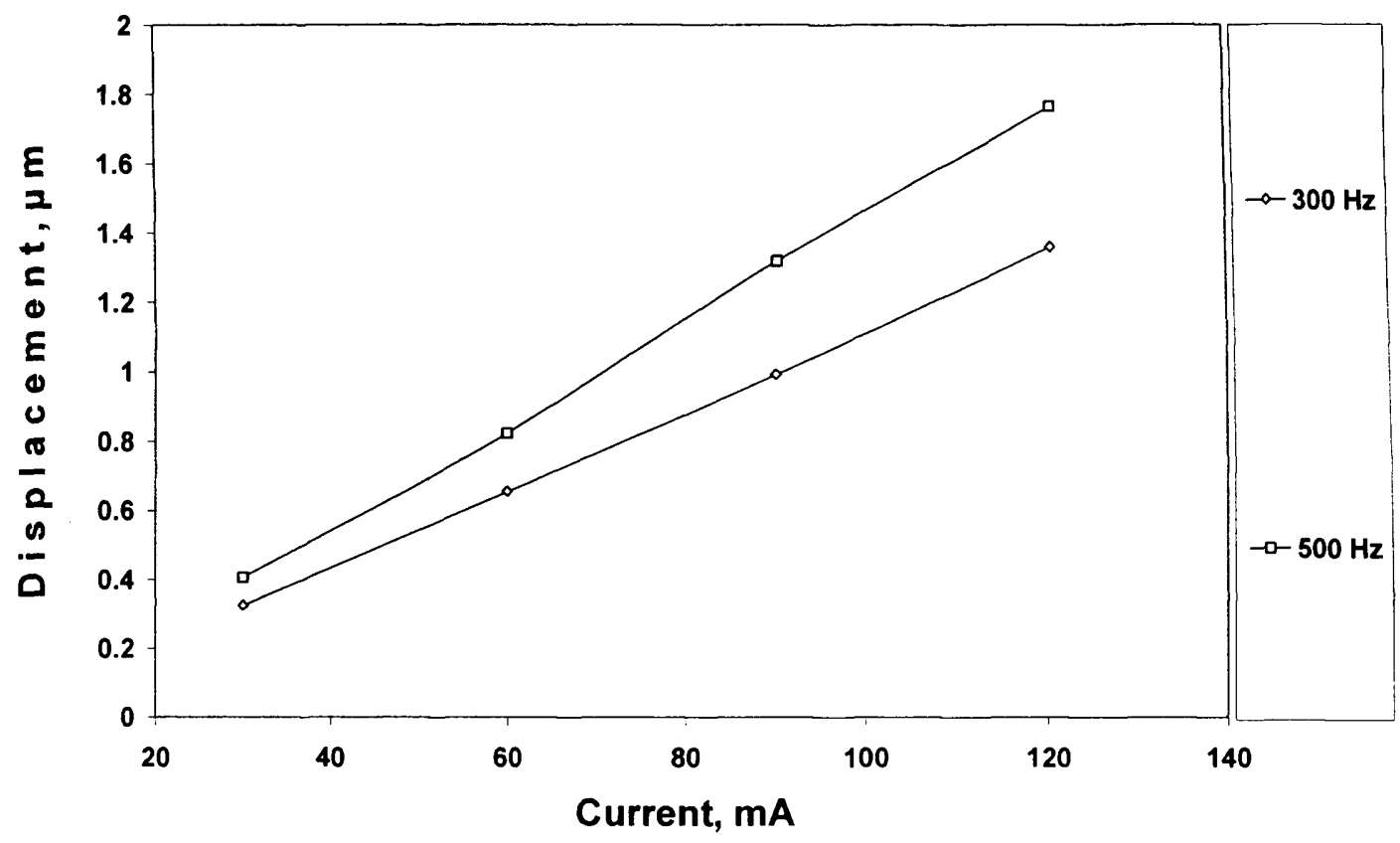

Figure 7.15: Displacement versus current curves for the moving coil configuration of the transducer speaker with excitation currents varying from $30 \mathrm{~mA}$ to $120 \mathrm{~mA}$ at frequencies $300 \mathrm{~Hz}$ and $500 \mathrm{~Hz}$

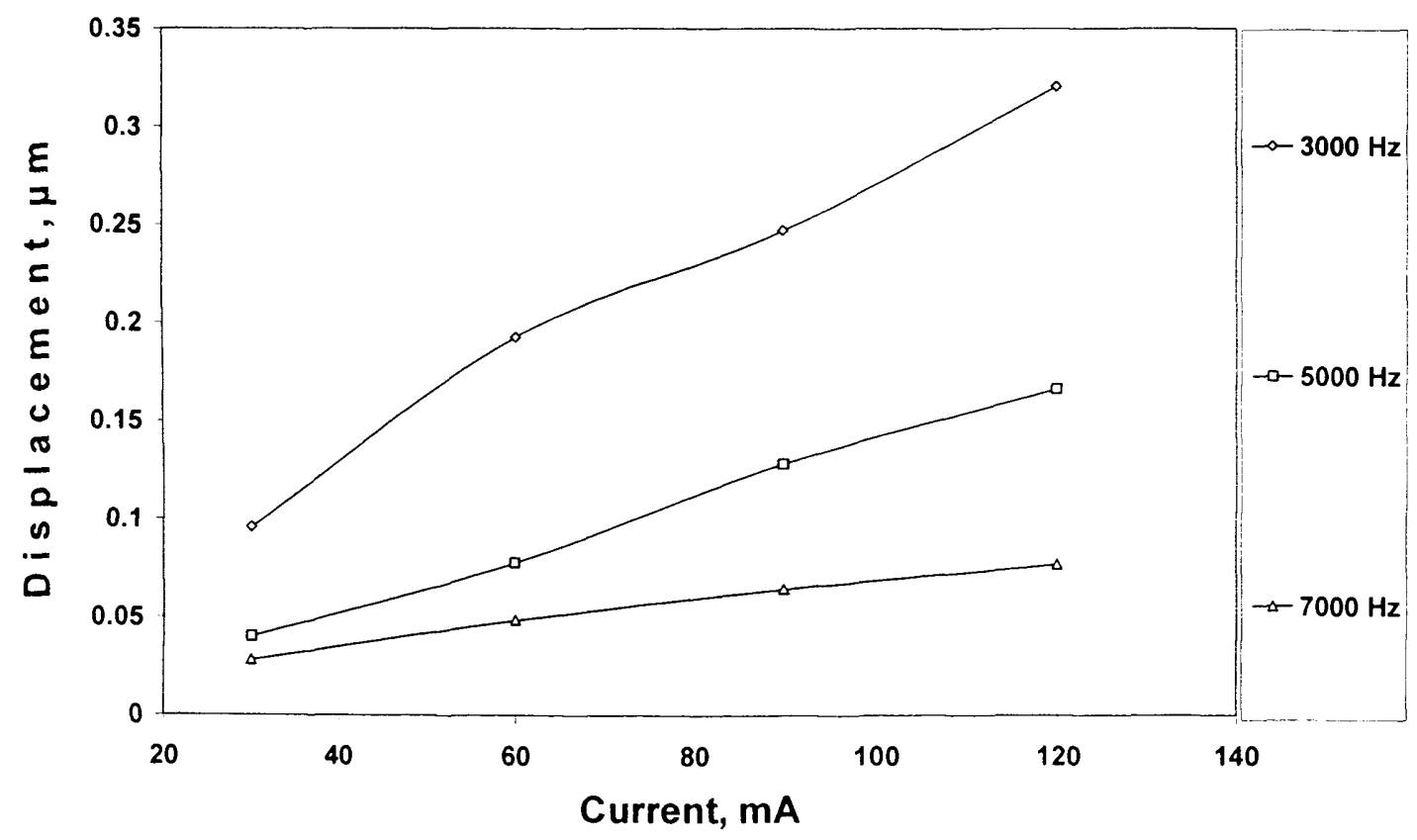

Figure 7.16: Displacement versus current curves for the moving coil configuration of the transducer speaker with excitation currents varying from $30 \mathrm{~mA}$ to $120 \mathrm{~mA}$ at frequencies $3 \mathrm{kHz}, 5 \mathrm{kHz}$ and $7 \mathrm{kHz}$ 


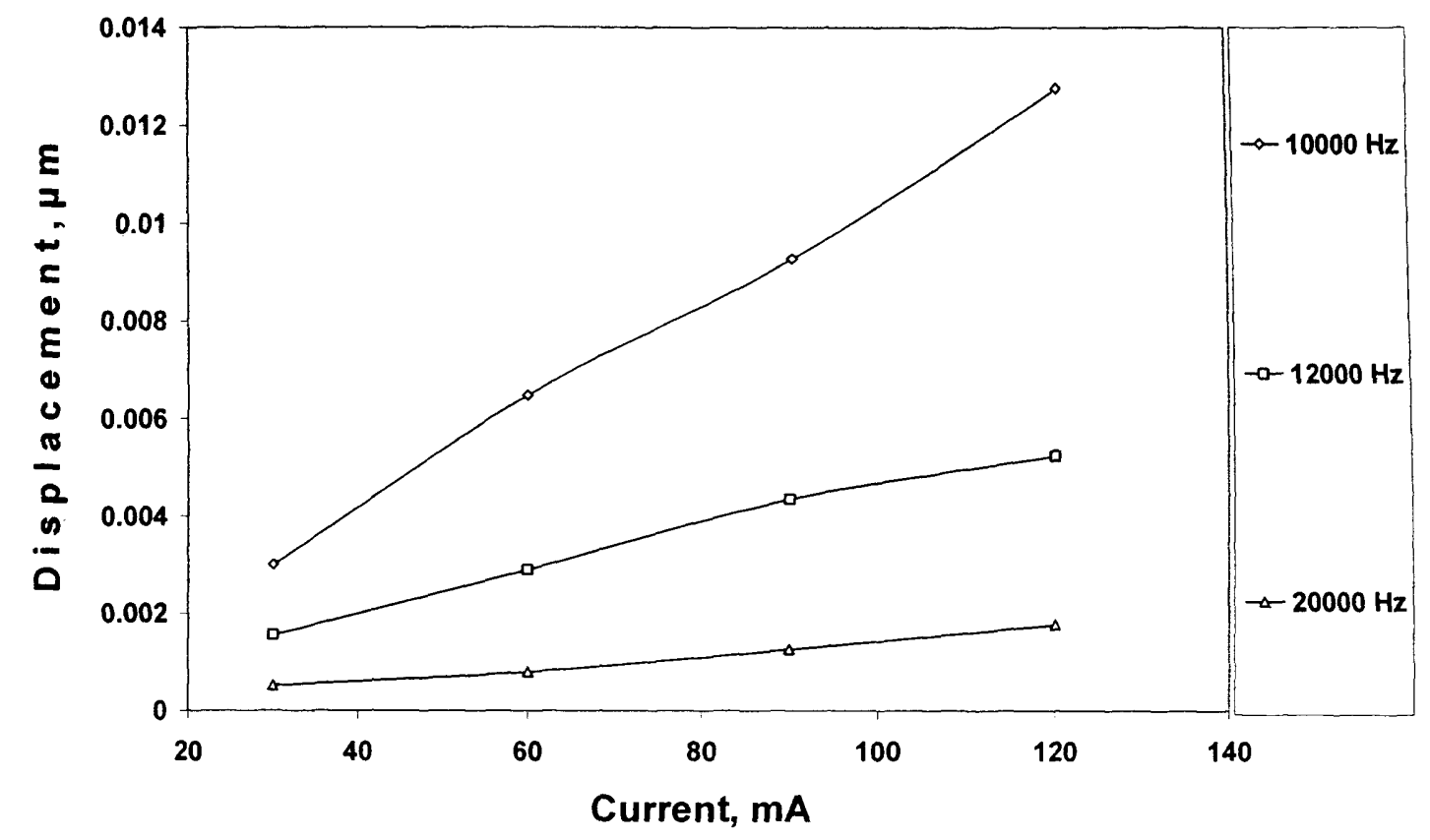

Figure 7.17: Displacement versus current curves for the moving coil configuration of the transducer speaker with excitation currents varying from $30 \mathrm{~mA}$ to $120 \mathrm{~mA}$ at frequencies $10 \mathrm{kHz}, 12 \mathrm{kHz}$ and $20 \mathrm{kHz}$

The displacement versus current curves (Figs. 7.15, 7.16 and 7.17) for the moving coil configuration shows that this configuration, unlike the moving magnet configuration, exhibited a linear current versus displacement characteristic. The linear relationship was observed for various frequency ranges and excitation currents. The main difference between the moving magnet and the moving coil configuration is that for the moving magnet configuration, non-linearity was introduced in the displacement versus current relationship for higher excitation currents at low frequency. However, in the case of a moving coil configuration, no such non-linearity was visible in the low frequency region. This was again due to the reduced mass of moving panel which extended the range for maximum excursion of the diaphragm and hence linear displacement versus current relationship was observed for higher excitation currents. In the mid-range and in the high frequency region, the linearity was observed both for low and high excitation currents. 


\subsubsection{Acoustic Intensity versus Frequency Response}

Figure 7.18 shows the acoustic intensity versus frequency response for the moving coil configuration of the transducer speaker. The following figure shows the acoustic power radiated by vibrating diaphragm at various frequencies in the audible frequency range. The acoustic intensity response also indicated the suitability of a transducer speaker for a given frequency range.

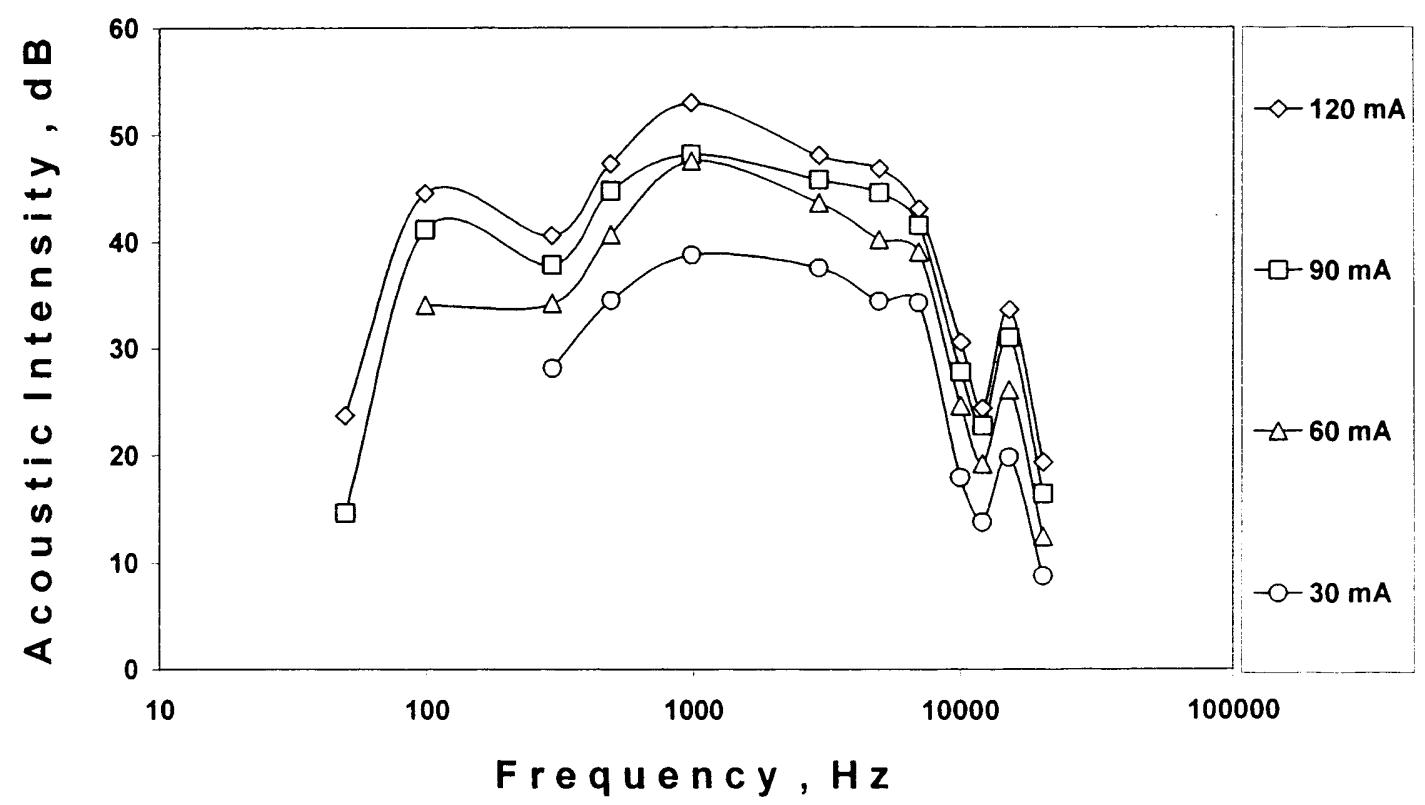

Figure 7.18: Acoustic intensity curves for the moving coil configuration of the transducer speaker showing acoustic intensity in decibel (dB) for frequencies varying from $20 \mathrm{~Hz}$ to $20 \mathrm{kHz}$ at various excitation currents ranging from $30 \mathrm{~mA}$ to $120 \mathrm{~mA}$

The acoustic intensity curve (Fig. 7.18) for the moving coil configuration of the miniature transducer speaker indicates that the lighter diaphragm in this device configuration has resulted in a flatter acoustic response compared to that of a moving magnet configuration. The flatter frequency response was achieved for a broad range of the audio frequency spectrum $(100 \mathrm{~Hz}$ to $8 \mathrm{KHz})$. This was clearly an indication of the improvement of the transducer speaker in terms of the frequency response. Therefore the moving coil configuration having a vibrating diaphragm with reduced mass could produce flatter frequency response and also higher displacements. 


\subsubsection{Resonance Curves}

The displacement profile of a transducer speaker along with the phase characteristics over the whole audio frequency range shows the presence of resonance in the transducer's operation. The operation characteristics of a transducer speaker system can be affected by resonance and hence it is important to identify the resonant peaks in the system. Figures 7.19 and 7.20 show the displacement versus frequency and phase versus frequency curves respectively for the moving coil configuration of the transducer speaker.

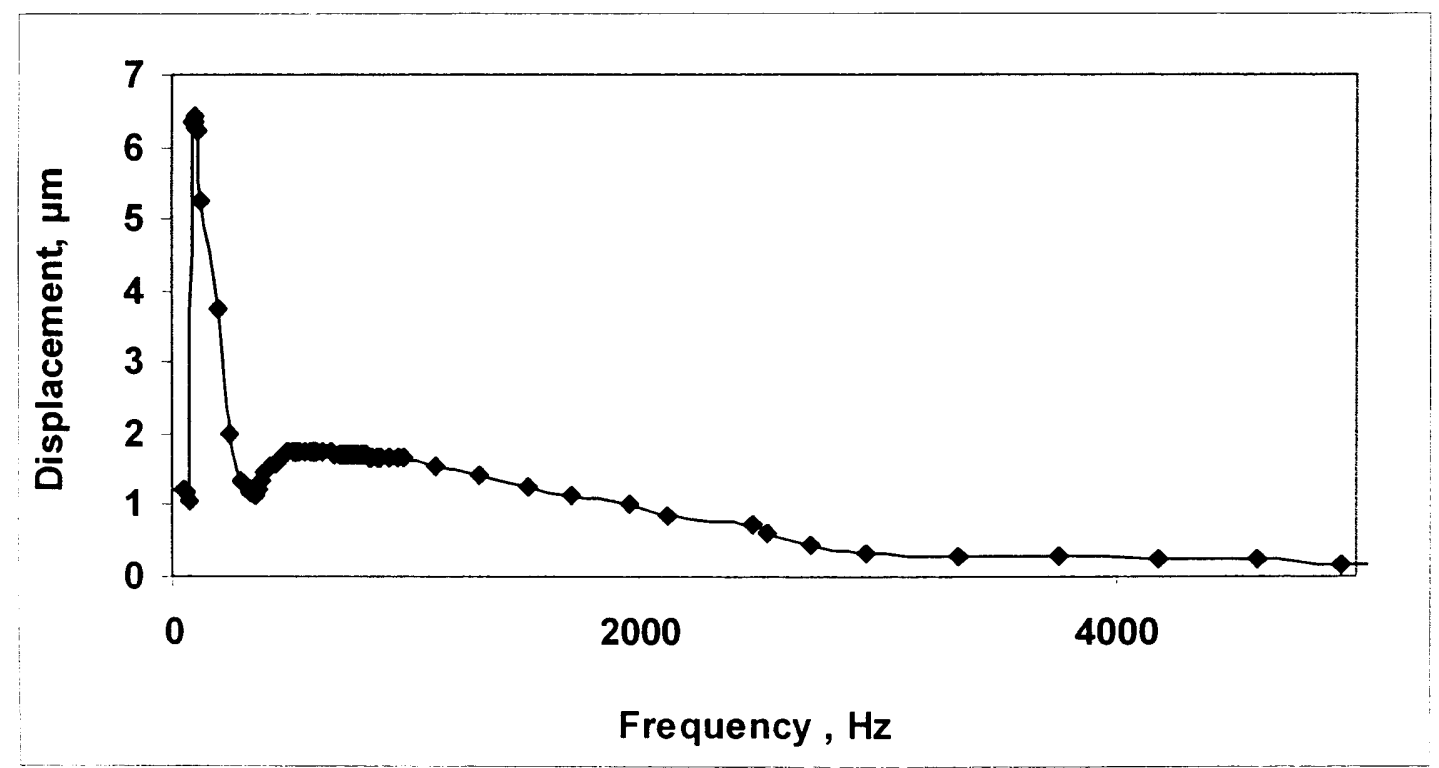

Figure 7.19: Displacement magnitude curve of the moving coil configuration of the transducer speaker showing resonances in the lower audio frequencies $(20 \mathrm{~Hz}$ to 5 $\mathrm{kHz}$ ). The displacement magnitudes were measured at an excitation current of 120 $m A$. 


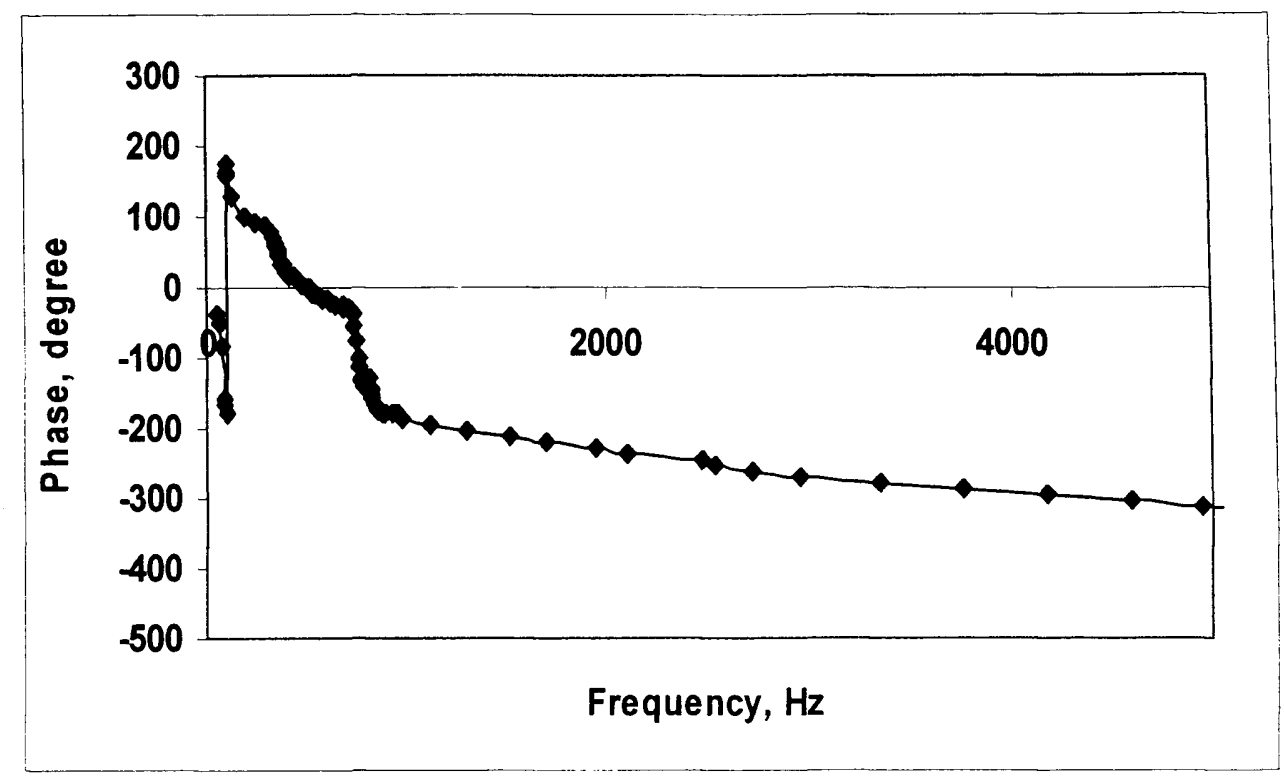

Figure 7.20: Phase curve of the moving coil configuration of the transducer speaker showing resonances in the lower audio frequencies $(20 \mathrm{~Hz}$ to $5 \mathrm{kHz})$. The phase information was recorded at an excitation current of $120 \mathrm{~mA}$.

The magnitude of the displacement and the corresponding phase indicated that there were resonant peaks in the low-frequency region. The resonant peak was observed at $100 \mathrm{~Hz}$ in the magnitude curve for the moving coil configuration. The corresponding phase response also indicated that the resonance occurred at $100 \mathrm{~Hz}$. However, in the high-frequency region no additional resonances were observed. Therefore, apart from the low-frequency end of the audio spectrum, the moving coil transducer speaker could reproduce a linear sound output across the whole audible frequency range.

\subsubsection{Acoustic Intensity versus Current Response}

The acoustic intensity versus current responses showed the linearity of acoustic intensity with excitation currents at various frequencies. In order to deliver acoustic power in proportion to the driving current over the whole audio frequency range and also to obtain a predictable and steady response, a linear acoustic intensity versus current relationship is expected from an ideal loudspeaker. Figures 7.21, 7.22 and 7.23 show the acoustic intensity versus driving current at low, medium and high frequencies, respectively, for the moving coil configuration of the transducer speaker. 


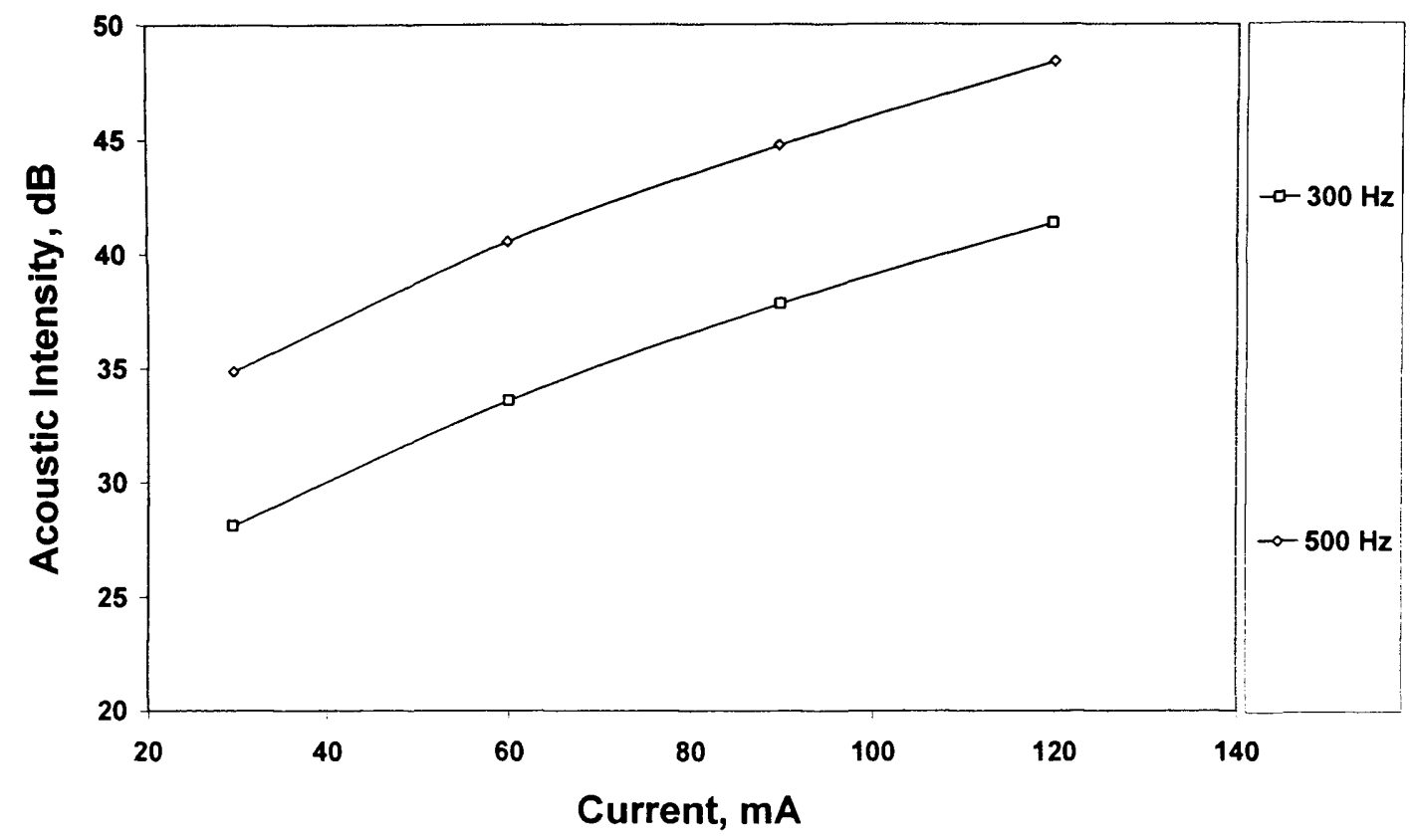

Figure 7.21: Acoustic intensity versus current curves for the moving coil configuration of the transducer speaker showing acoustic intensities with excitation currents varying from $30 \mathrm{~mA}$ to $120 \mathrm{~mA}$ at frequencies $300 \mathrm{~Hz}$ and $500 \mathrm{~Hz}$

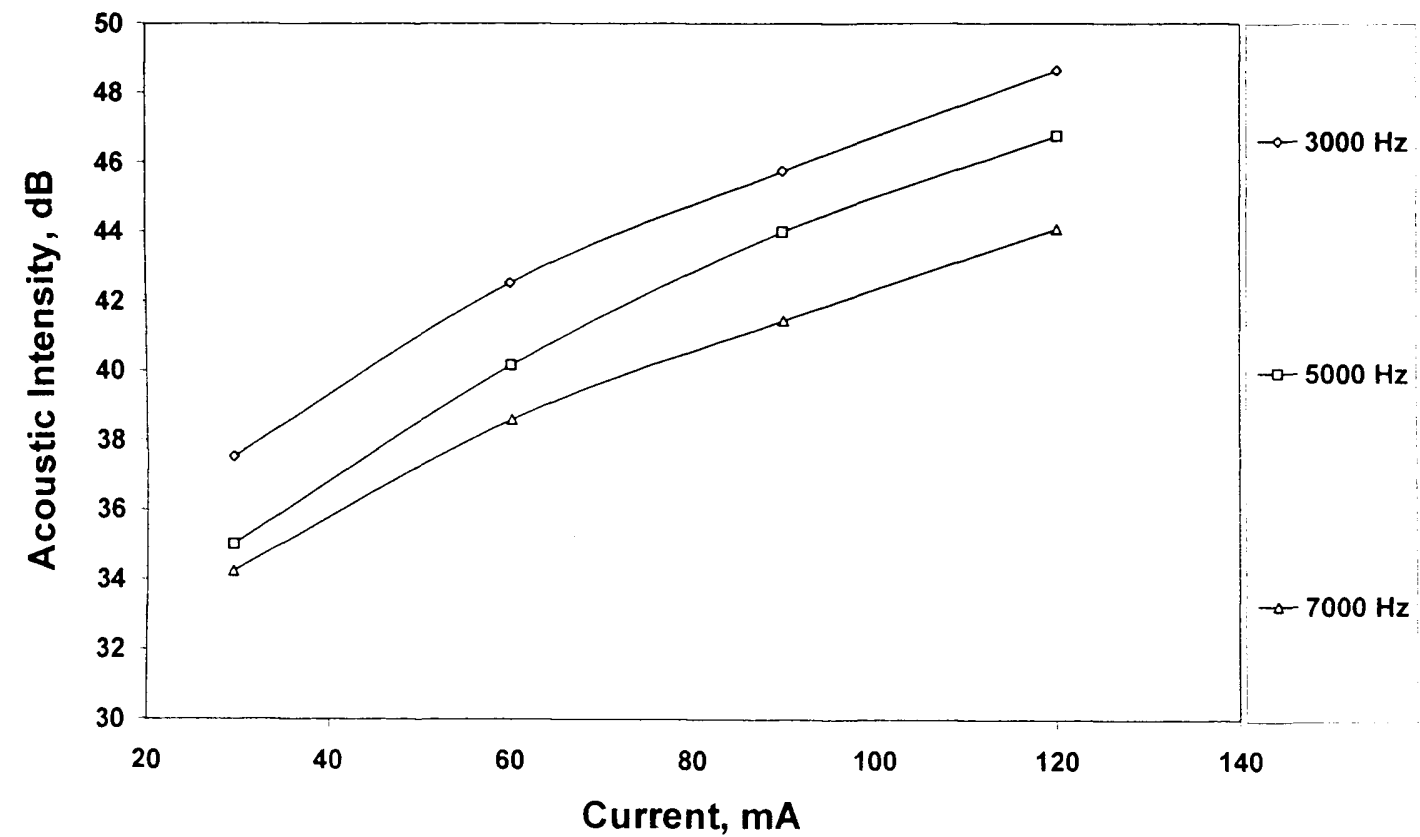

Figure 7.22: Acoustic intensity versus current curves for the moving coil configuration of the transducer speaker showing acoustic intensities with excitation currents varying from $30 \mathrm{~mA}$ to $120 \mathrm{~mA}$ at frequencies $3 \mathrm{kHz}, 5 \mathrm{kHz}$ and $7 \mathrm{kHz}$ 


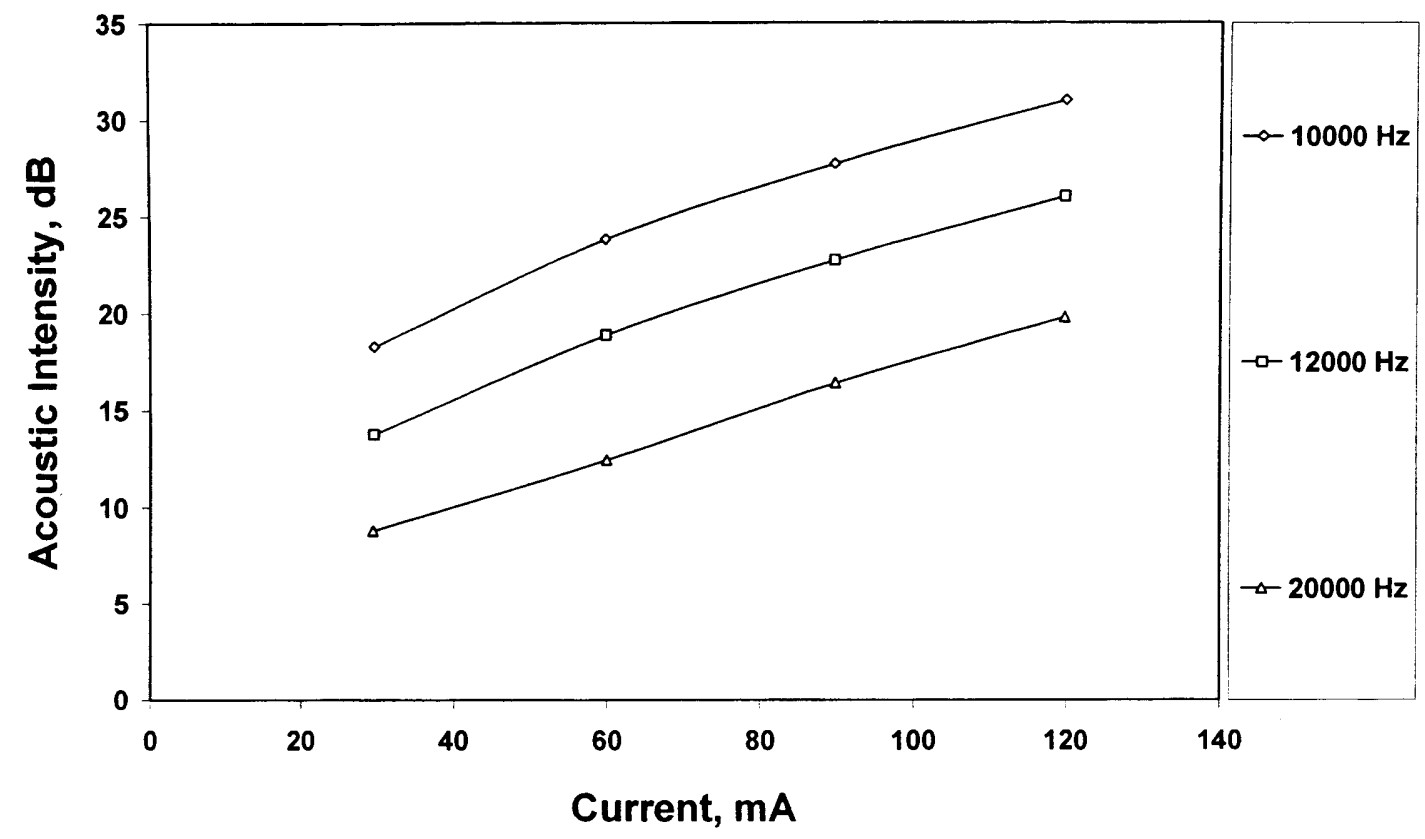

Figure 7.23: Acoustic intensity versus current curves for the moving coil configuration of the transducer speaker showing acoustic intensities with excitation currents varying from $30 \mathrm{~mA}$ to $120 \mathrm{~mA}$ at frequencies $12 \mathrm{kHz}, 15 \mathrm{kHz}$ and $20 \mathrm{kHz}$

Using the curve fitting technique, the figures show that the relationship between the acoustic intensity and the excitation current for the moving coil arrangement closely matched to that of a linear approximation (0.99). However, the data points were too few to conclude about the nature of the relationship. Although, taking into account the experimental error and the current versus displacement relationships, it can be approximated that the relationship between the current and the acoustic intensity was linear. 


\subsection{Results for Moving Magnet with a Non-Magnetic Bottom Layer}

A third set of results was recorded for the moving magnet with a 'non-magnetic bottom layer' combination. This specific arrangement of the transducer had exactly the same configuration as that of a moving magnet one but instead of having an amorphous bottom layer, this arrangement employed a non-magnetic material (plastic) as the bottom layer. Results were recorded for the miniature transducer system to understand the effect of having an amorphous panel at the bottom of the transducer. The top amorphous layer, apart from holding the permanent magnet, was also providing a flux path for the magnetic circuit. However, since the bottom amorphous layer was not contributing significantly in terms of flux distribution in the magnetic circuit (Fig. 7.24) because of the existing air-gap between the top and the bottom layer, a non-magnetic material was considered for the bottom layer to observe the effect of having a non-magnetic second panel.

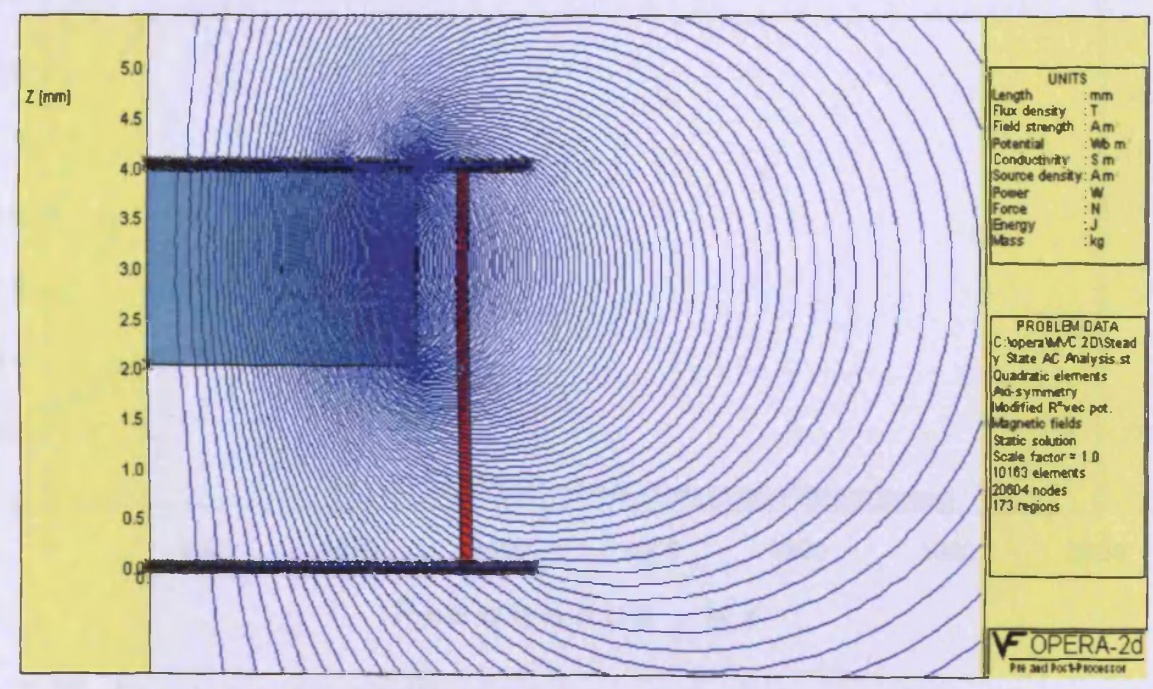

Figure 7.24: Simulation result indicating the inefficiency of the base layer in terms of flux distribution due to air gap 


\subsubsection{Displacement versus Frequency Responses}

The moving magnet with a non-magnetic bottom layer configuration of the transducer speaker was tested for frequency responses. Figures 7.25 and 7.26 show the displacement versus frequency responses of the transducer in the audio frequency range. In order to obtain the displacement characteristics at various frequencies, the vibration of the diaphragm which was attached to the permanent magnet was measured using a laser Doppler vibrometer. The laser from the vibrometer was incident on the front panel of the transducer speaker while the frequency was varied from $20 \mathrm{~Hz}$ to $20 \mathrm{kHz}$. At each observed frequency, the excitation current was varied from $30 \mathrm{~mA}$ to $120 \mathrm{~mA}$.

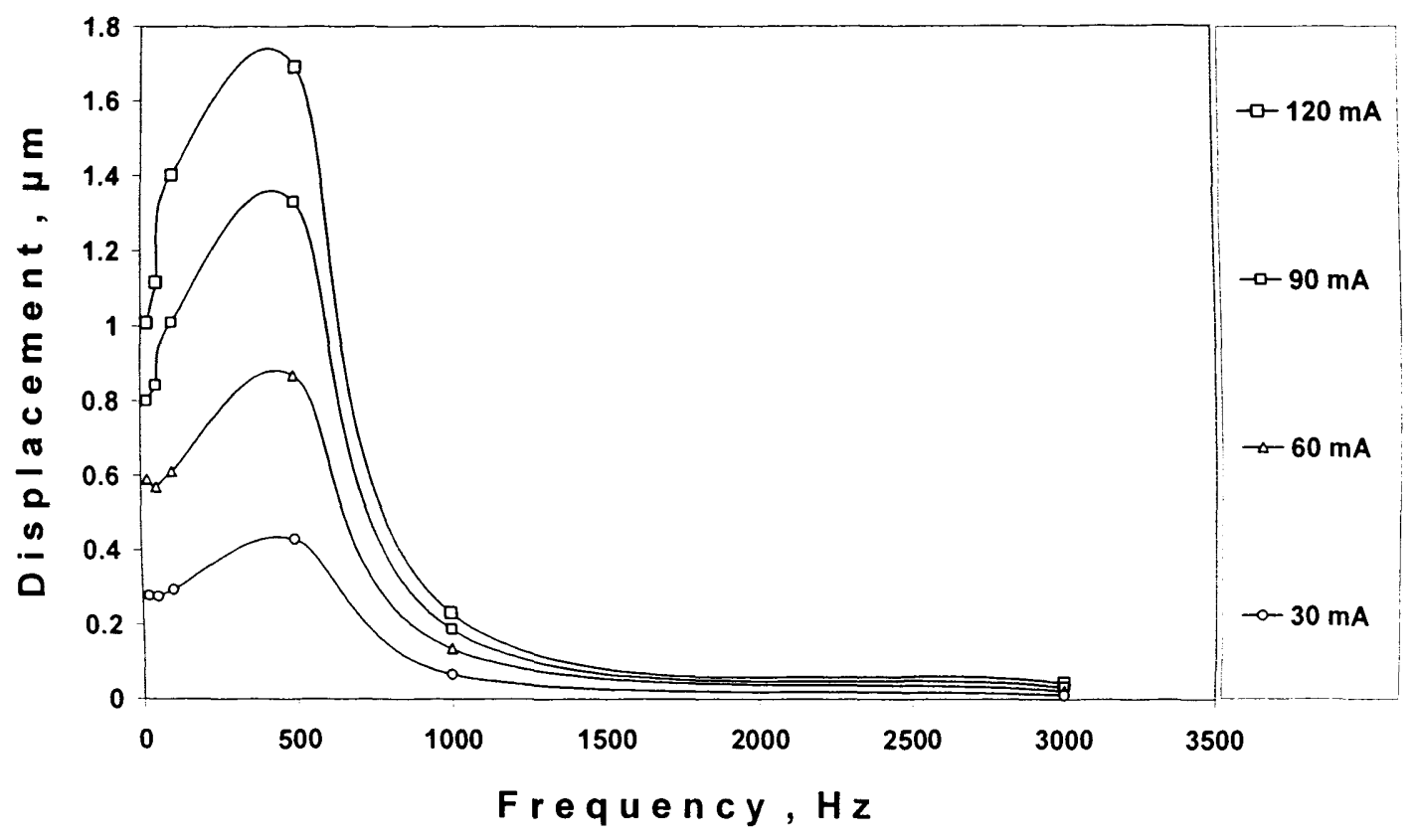

Figure 7.25: Displacement profile (non-resonant) of the moving magnet with a nonmagnetic bottom layer configuration of the transducer speaker showing displacements in the frequency range of $20 \mathrm{~Hz}$ to $3 \mathrm{kHz}$ with excitation currents varying from 30 $m A$ to $120 \mathrm{~mA}$. Displacements measured with laser incident on the centre of the vibrating diaphragm. 


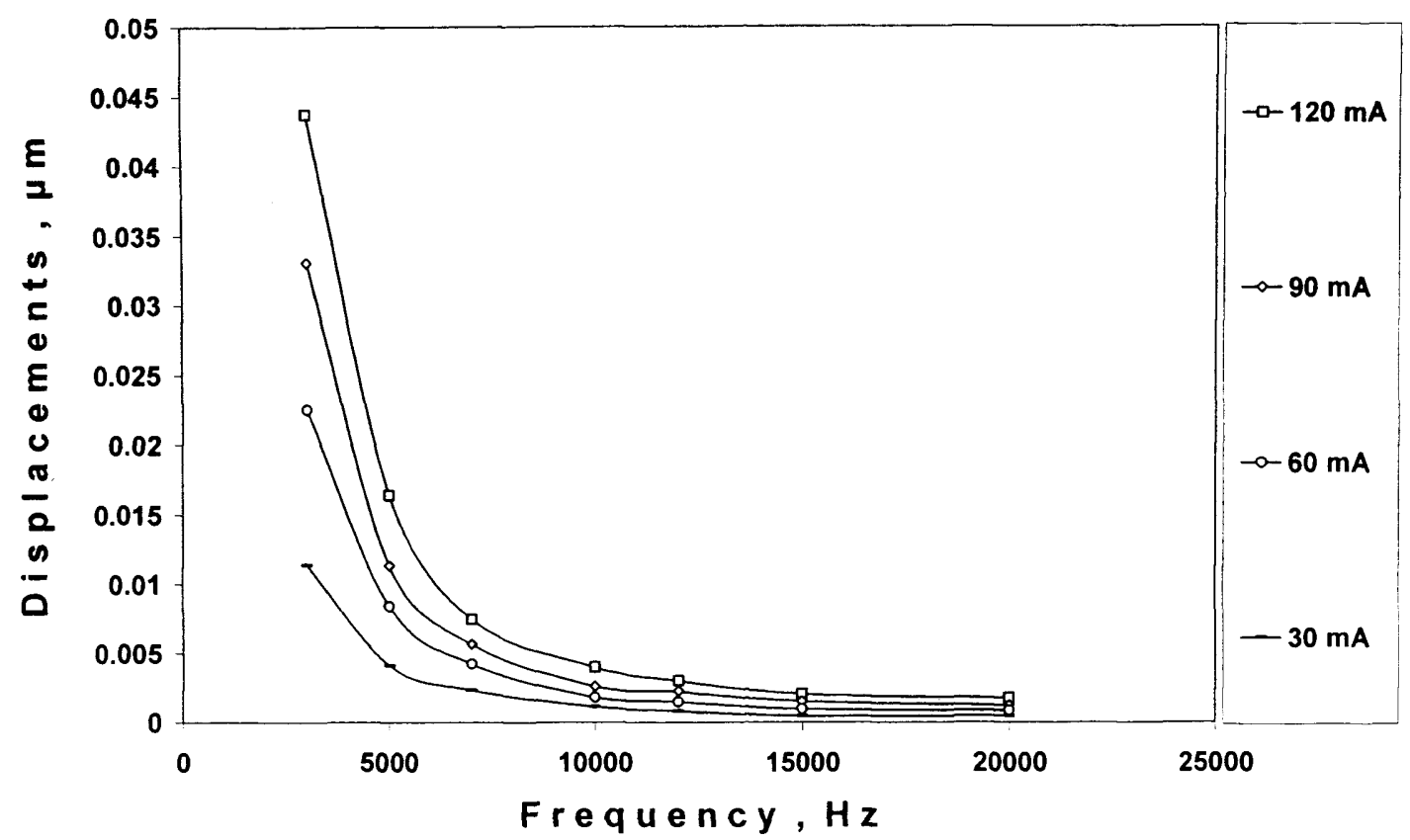

Figure 7.26: Displacement profile (non-resonant) of the moving magnet with a nonmagnetic bottom layer configuration of the transducer speaker showing displacements in the frequency range of $3 \mathrm{kHz}$ to $20 \mathrm{kHz}$ with excitation currents varying from 30 $m A$ to $120 \mathrm{~mA}$. Displacements measured with laser incident on the centre of the vibrating diaphragm.

The displacement versus frequency responses (Figs. 7.25 and 7.26) for the moving magnet with a 'non-magnetic' bottom layer combination showed that the frequency responses were almost the same as that of a moving magnet configuration. However, in the low frequency region (Fig. 7.25), especially below $1 \mathrm{kHz}$, unstable responses were observed for this transducer configuration. The reason for such unstable behaviours at the low end of the audio frequency spectrum was due to the lack of a proper return mechanism for the vibrating panel. In a moving magnet configuration, the top and the bottom amorphous layers attract each others and provide a holding force for the vibrating diaphragm. Since in this transducer configuration, in which the magnetic bottom panel was replaced with a non-magnetic one, there was no holding force or return mechanism between the top and bottom layer, the miniature transducer speaker was displaying unstable behaviour at the low end of the audio frequency where the excursion level is high. 
In the high frequency region (Fig. 7.26), this transducer configuration exhibited higher displacement characteristics than the moving magnet one (Fig. 7.2). The lack of a holding force between the top and bottom layer, as discussed earlier, meant that the vibrating front panel could move more independently and hence produced higher displacements. However, the displacements for this specific configuration were still lower than that of the moving coil configuration. This was because the vibrating panel for this configuration had the added weight of the permanent magnet attached to its surface. Whereas in the case of a moving coil configuration, the vibrating panel was lighter and therefore could produce higher displacements.

\subsubsection{Displacement versus Current Responses}

For the moving magnet with a non-magnetic bottom layer configuration of the transducer speaker, figures 7.27, 7.28 and 7.29 shows the variation of the displacement with excitation currents. The following figures explore whether the novel transducer can operate as a linear device in which the output is proportional to the input. A linear excitation displacement versus current relationship for a loudspeaker is useful since, in order to obtain a predictable steady response, the diaphragm of a speaker needs to move with amplitudes proportional to the driving current. 


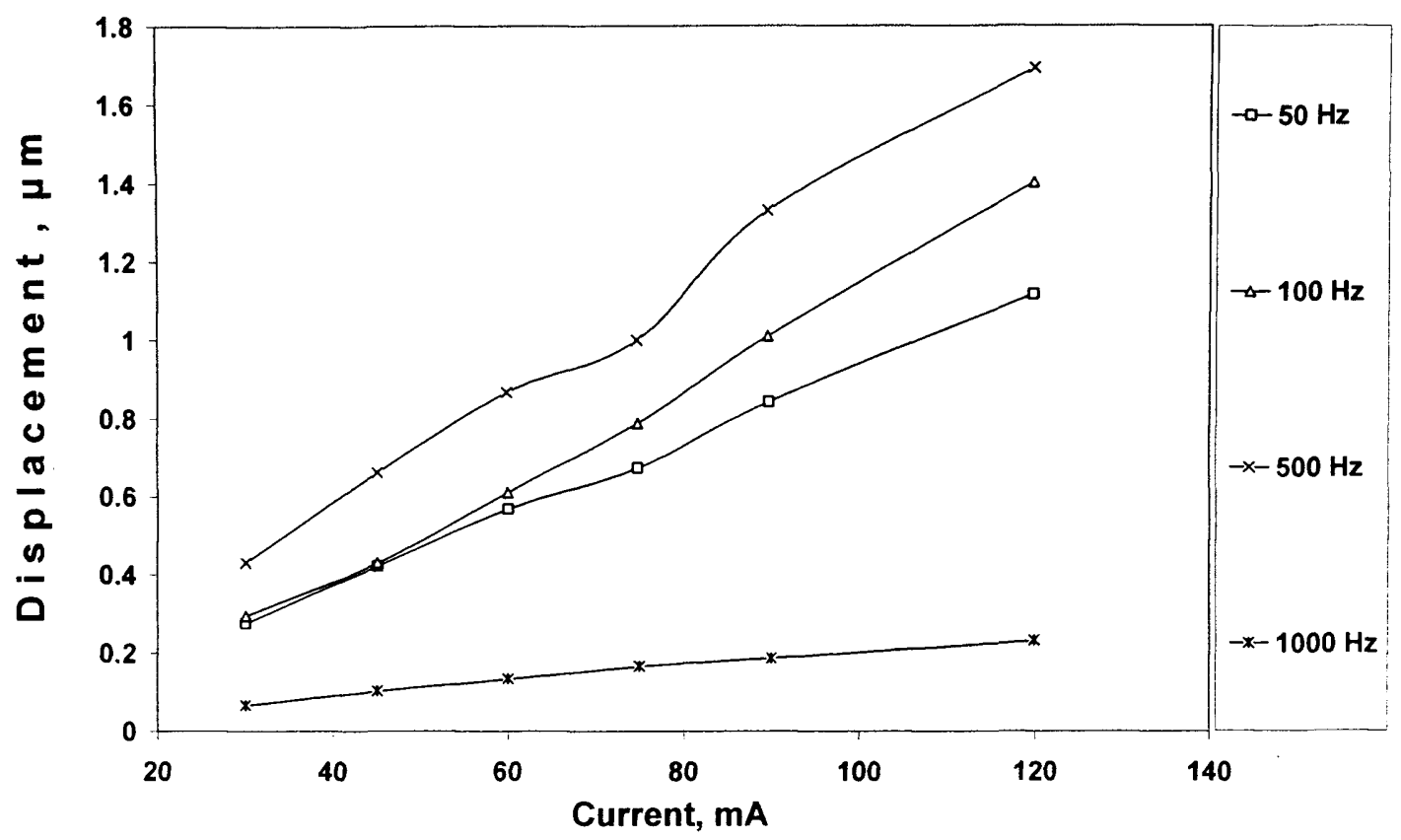

Figure 7.27: Displacement versus current curves for the moving magnet with a nonmagnetic bottom layer configuration of the transducer speaker with excitation currents varying from $30 \mathrm{~mA}$ to $120 \mathrm{~mA}$ at frequencies $50 \mathrm{~Hz}, 100 \mathrm{~Hz}, 500 \mathrm{~Hz}$ and $1 \mathrm{kHz}$

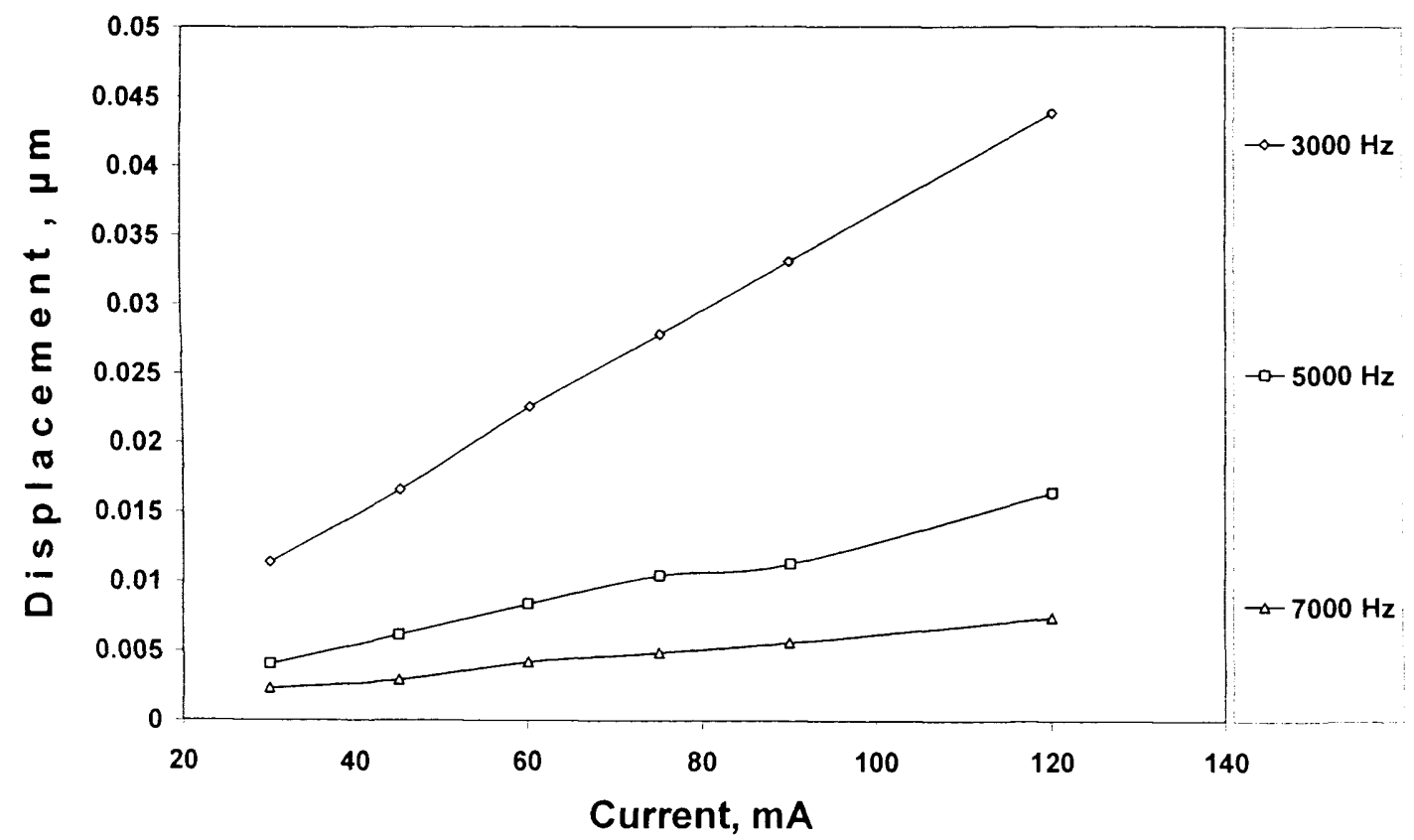

Figure 7.28: Displacement versus current curves for the moving magnet with a nonmagnetic bottom layer configuration of the transducer speaker with excitation currents varying from $30 \mathrm{~mA}$ to $120 \mathrm{~mA}$ at frequencies $3 \mathrm{kHz}, 5 \mathrm{kHz}$ and $7 \mathrm{kHz}$ 


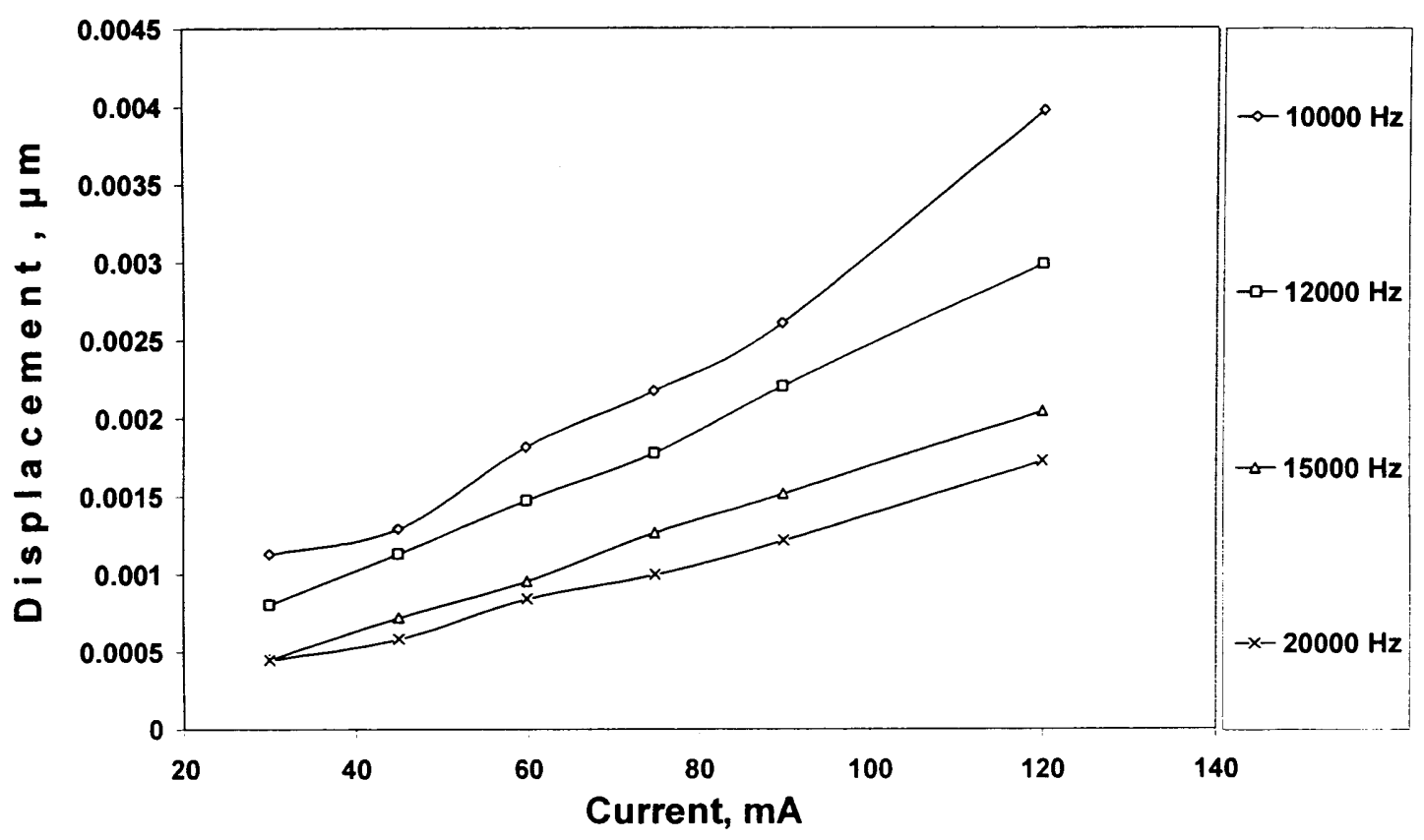

Figure 7.29: Displacement versus current curves for the moving magnet with a nonmagnetic bottom layer configuration of the transducer speaker with excitation currents varying from $30 \mathrm{~mA}$ to $120 \mathrm{~mA}$ at frequencies $10 \mathrm{kHz}, 12 \mathrm{kHz}, 15 \mathrm{kHz}$ and $20 \mathrm{kHz}$

The current versus displacements relationships (Figs. 7.27, 7.28 and 7.29) for the moving magnet with a 'non-magnetic' bottom layer showed that, in the low frequency region (Fig. 7.27) the transducer exhibited unstable and non-linear displacement characteristics. The reasons for such 'unstable' behaviour in the low frequency region is the high excursion at low frequencies and the lack of a magneto-mechanical return mechanism as discussed in the previous paragraphs. Although in the mid and high frequency range, the relationship between the excitation current and displacements was more linear compared to that in the low frequency region, the unstable displacements characteristics were visible throughout the whole audio frequency range. 


\subsubsection{Acoustic Intensity versus Frequency Response}

Figure 7.30 shows the acoustic intensity versus frequency response for the moving magnet with a non-magnetic bottom layer configuration of the transducer speaker. This figure shows the acoustic power radiated by vibrating diaphragm at various frequencies in the audible frequency range. The acoustic intensity response also indicated the suitability of this transducer speaker for a given frequency range.

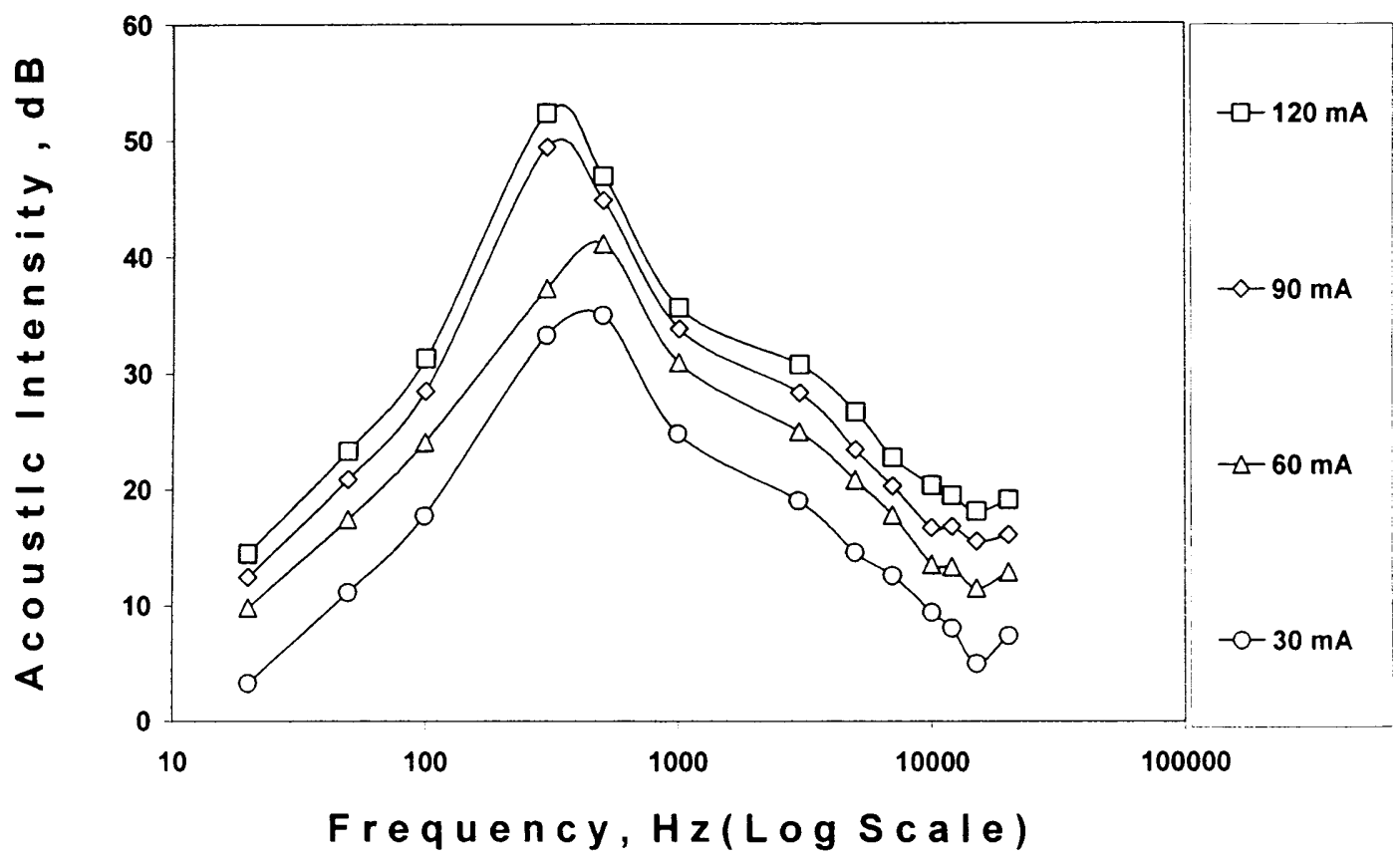

Figure 7.30: Acoustic intensity curves for the moving magnet with a non-magnetic bottom layer configuration of the transducer speaker showing acoustic intensity in decibel $(\mathrm{dB})$ for frequencies varying from $20 \mathrm{~Hz}$ to $20 \mathrm{kHz}$ at various excitation currents ranging from $30 \mathrm{~mA}$ to $120 \mathrm{~mA}$

The acoustic intensity versus frequency curve (Fig. 7.30) indicates that this transducer configuration had a similar acoustic intensity response as that of a moving magnet one. The displacement versus frequency responses (Fig. 7.25 and 7.26) of this transducer showed a gradual decrease in displacements at low frequency (i.e. below 1 $\mathrm{kHz}$ ) and a sharp decrease in displacements as the frequency increased in the high frequency region (above $1 \mathrm{kHz}$ ). Theoretically, acoustic intensity at a certain 
frequency is calculated by a term that consists of a product of that specific frequency and the respective displacement [2]. Therefore the acoustic intensity curve showed a gradual increase in intensity below $1 \mathrm{kHz}$ and a gradual decrease in intensity above 1 $\mathrm{kHz}$.

\subsubsection{Resonance Curves}

The displacement profile of the transducer speaker along with the phase characteristics over the whole audio frequency range showed the presence of resonance in the transducer's operation. The operation characteristics of a transducer speaker system can be affected by resonances and hence it is important to identify the resonant peaks in the system. The resonant curves (Figs. 7.31 and 7.32) showed the resonant behaviour for the moving magnet with non-magnetic layer configuration within the audible frequency range. Figure 7.31 and 7.32 show the displacement versus frequency and phase versus frequency curves respectively.

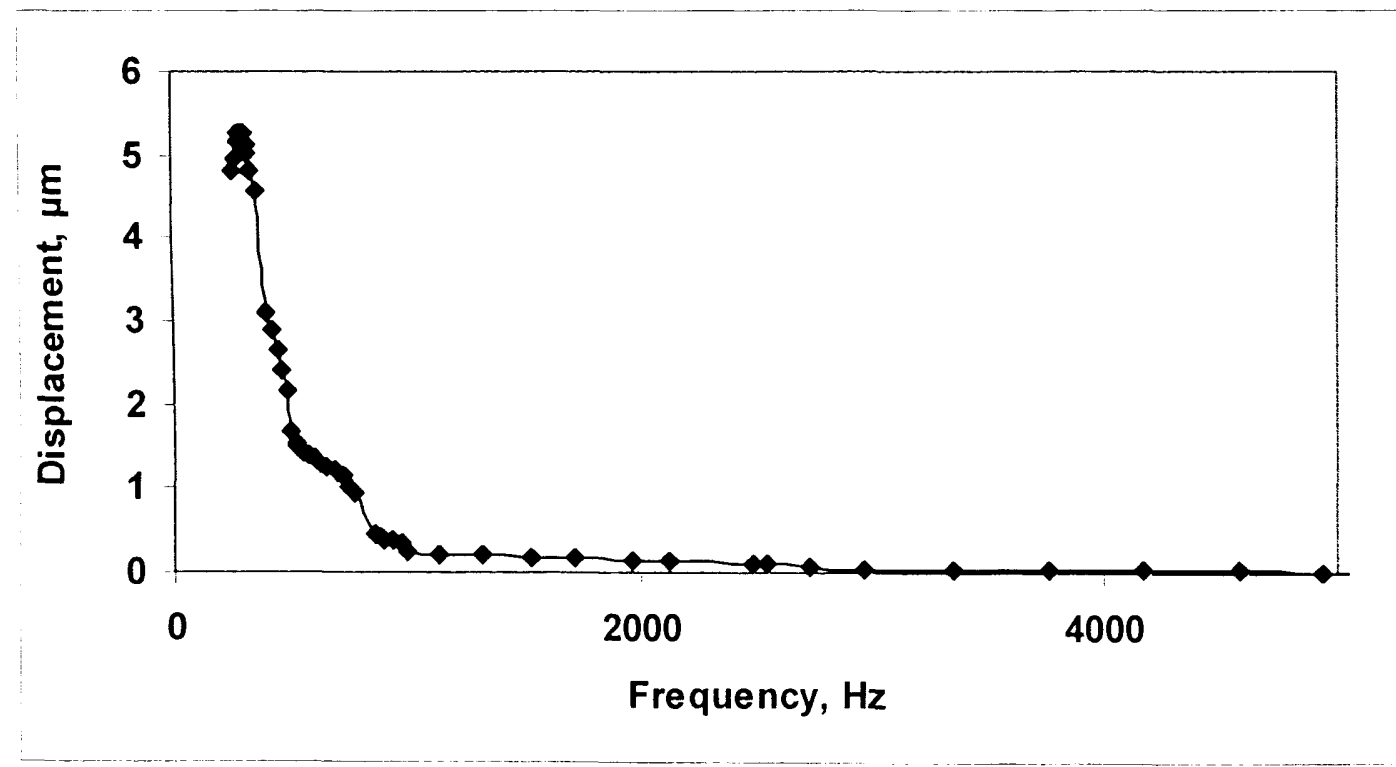

Figure 7.31: Displacement magnitude curve of the moving magnet with a nonmagnetic bottom layer configuration of the transducer speaker showing resonances in the lower audio frequencies $(20 \mathrm{~Hz}$ to $5 \mathrm{kHz}$ ). Resonance was not observed beyond this frequency range. The displacement magnitudes were measured at an excitation current of $120 \mathrm{~mA}$. 


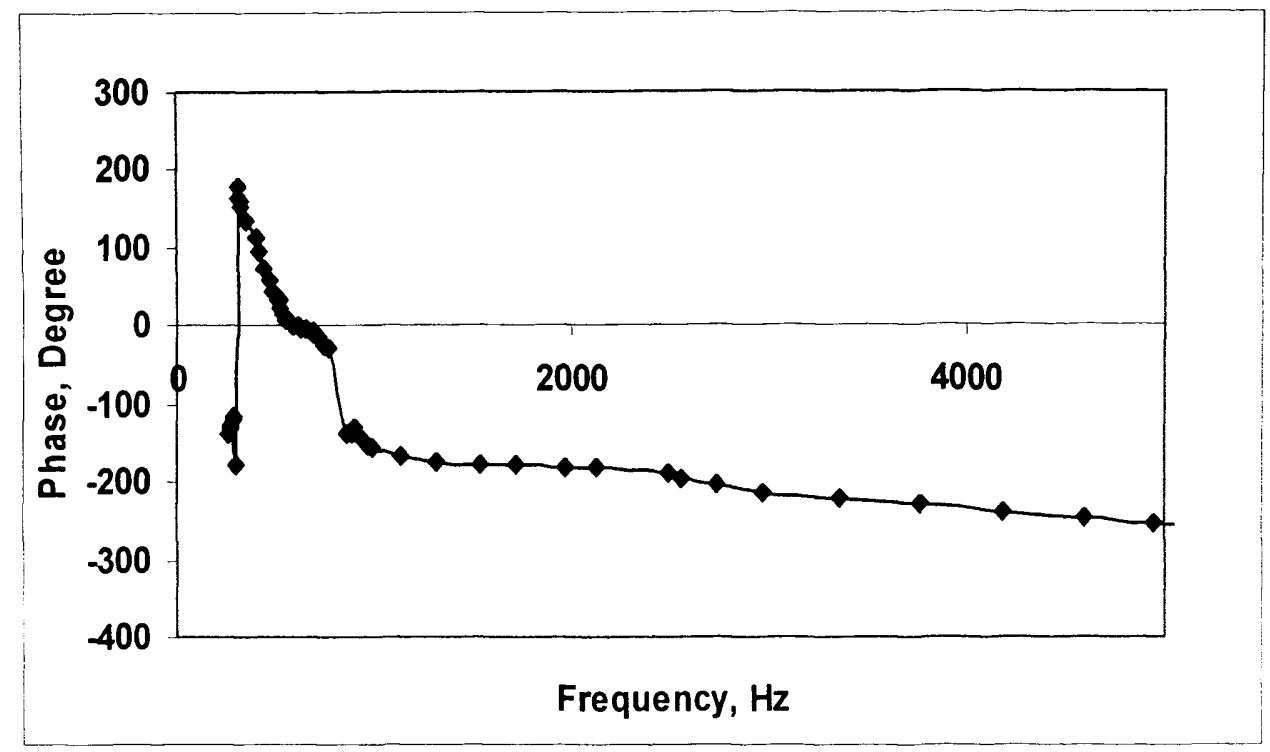

Figure 7.32: Phase curve of the moving magnet with a non-magnetic bottom layer configuration of the transducer speaker showing resonances in the lower audio frequencies $(20 \mathrm{~Hz}$ to $5 \mathrm{kHz}$ ). Resonance was not observed beyond this frequency range. The phase information was recorded at an excitation current of $120 \mathrm{~mA}$.

The magnitude of the displacement and the corresponding phase indicate that there are resonant peaks in the low-frequency region. The resonant peak was observed around $300 \mathrm{~Hz}$ in the magnitude curve for this configuration. The corresponding phase response also indicated that the resonance occurred around $300 \mathrm{~Hz}$. However, as in the case of other two device configurations, no additional resonances were observed in the high-frequency region. Therefore, apart from the low-frequency end of the audio spectrum, this configuration of the novel transducer speaker could reproduce sound output without the effect of resonance. 


\subsubsection{Acoustic Intensity versus Current Response}

The acoustic intensity versus current responses for the moving magnet with a nonmagnetic bottom layer configuration of the transducer speaker showed the linearity of acoustic intensity with excitation currents at various frequencies. Figures 7.33, 7.34 and 7.35 show the acoustic intensity versus current at low, medium and high frequencies, respectively, for the moving magnet with a non-magnetic bottom layer configuration. In order to deliver acoustic power in proportion to the driving current over the whole audio frequency range and also to obtain a predictable and steady response, a linear acoustic intensity versus current relationship is expected from an ideal loudspeaker.

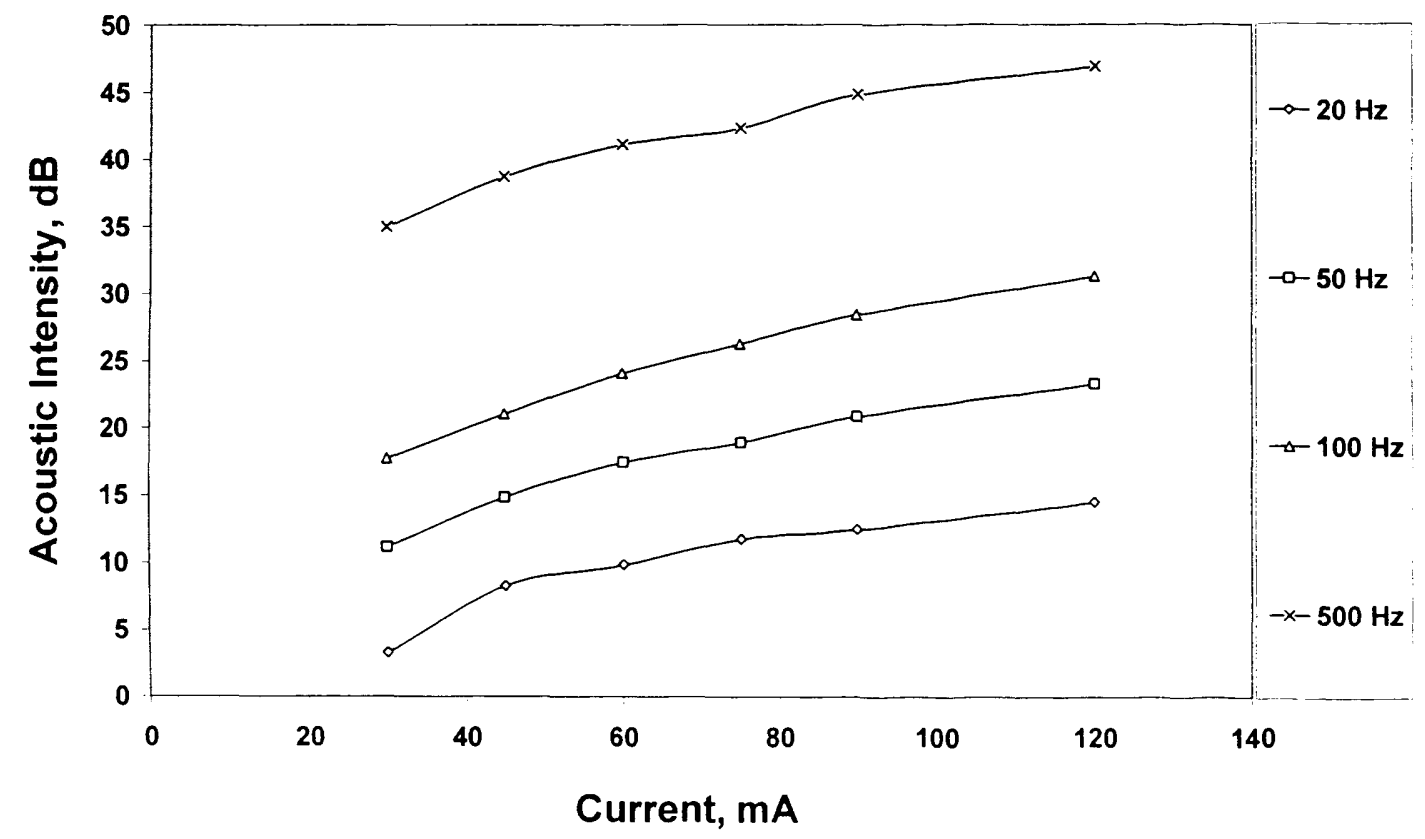

Figure 7.33: Acoustic intensity versus current curves for the moving magnet with a non-magnetic bottom layer configuration of the transducer speaker showing acoustic intensities with excitation currents varying from $30 \mathrm{~mA}$ to $120 \mathrm{~mA}$ at frequencies 20 $\mathrm{Hz}, 50 \mathrm{~Hz}, 100 \mathrm{~Hz}$ and $500 \mathrm{~Hz}$ 


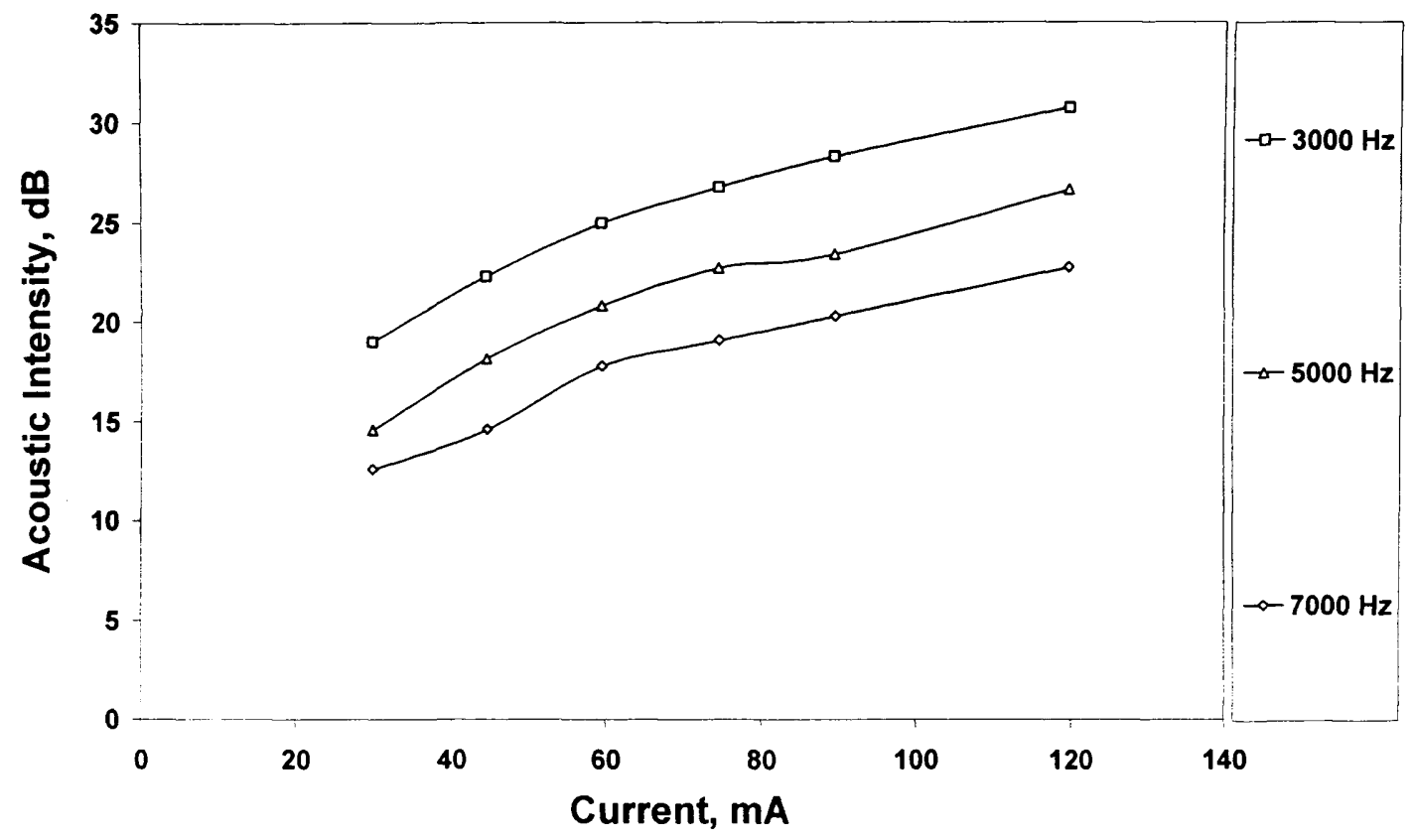

Figure 7.34: Acoustic intensity versus current curves for the moving magnet with a non-magnetic bottom layer configuration of the transducer speaker showing acoustic intensities with excitation currents varying from $30 \mathrm{~mA}$ to $120 \mathrm{~mA}$ at frequencies 3 $\mathrm{kHz}, 5 \mathrm{kHz}$ and $7 \mathrm{kHz}$

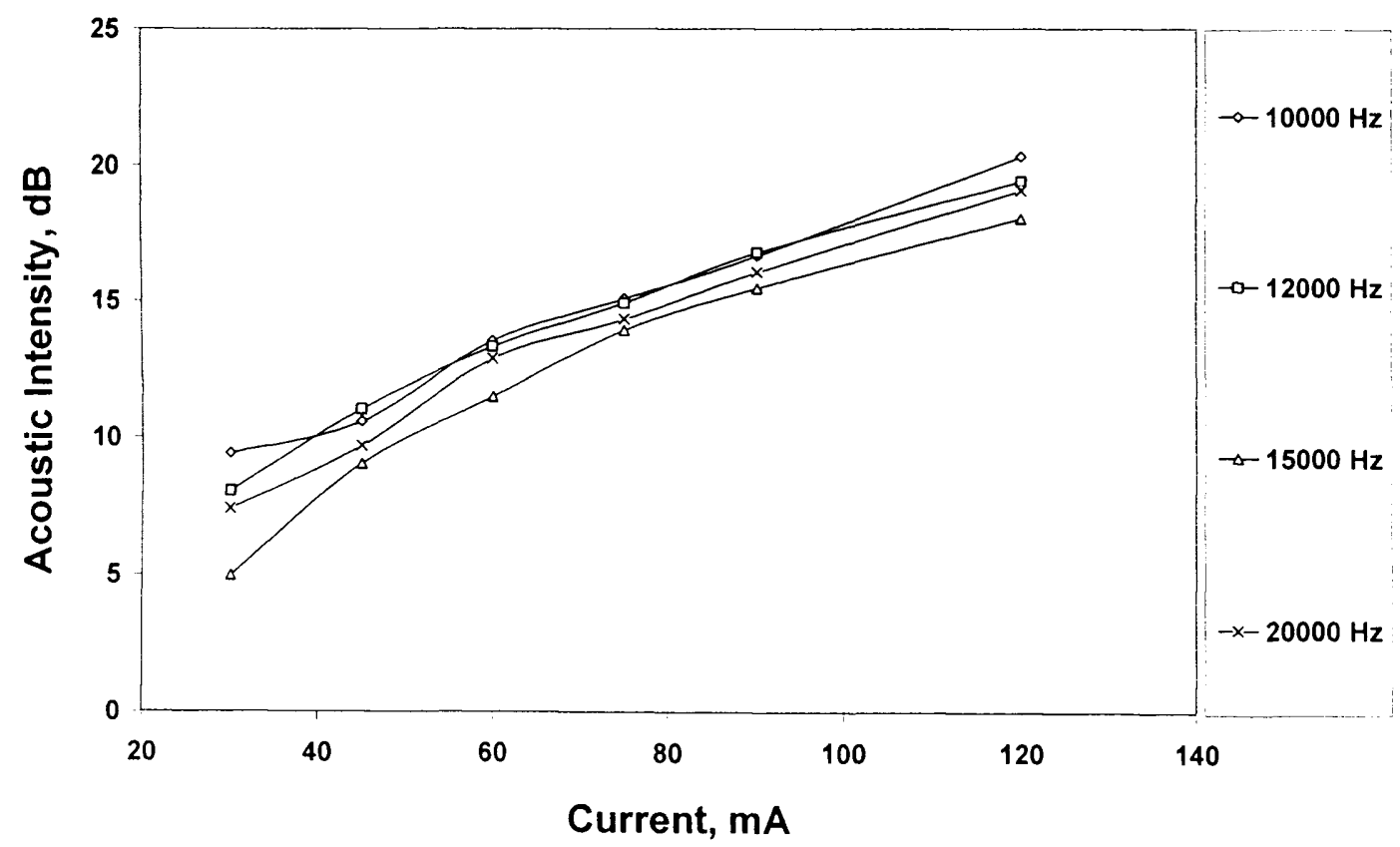

Figure 7.35: Acoustic intensity versus current curves for the moving magnet with a non-magnetic bottom layer configuration of the transducer speaker showing acoustic intensities with excitation currents varying from $30 \mathrm{~mA}$ to $120 \mathrm{~mA}$ at frequencies 10 $\mathrm{kHz}, 12 \mathrm{kHz}, 15 \mathrm{kHz}$ and $20 \mathrm{kHz}$ 
The curves for sound intensities (Figs. 7.33, 7.34 and 7.35) showed that the relationship between the current and the acoustic intensity was almost linear. These responses were also similar to that of the moving magnet configuration. The linear responses could be used to approximate the transducer as a linear device. However, the small amount of non-linearity which was visible at lower excitation currents was due to a lack of available driving force to displace the mass of the permanent magnet and the diaphragm. Experimental error should also be taken into account for these non-linearities. 


\subsection{Results for Moving Coil with a Non-Magnetic Diaphragm}

It was shown from the previous results that a non-magnetic layer introduces instability in the system as discussed in the section 7.4. However, a separate experiment was carried out on a miniature transducer speaker with a non-magnetic diaphragm in order to understand the effects of a magnetic panel. The miniature speaker under test was effectively a moving coil configuration but instead of having a magnetic diaphragm as used before, a non-magnetic material was used as the panel for the speaker. Figures 7.36 and 7.37 show the displacement versus frequency responses of this transducer configuration at high and low frequencies respectively.

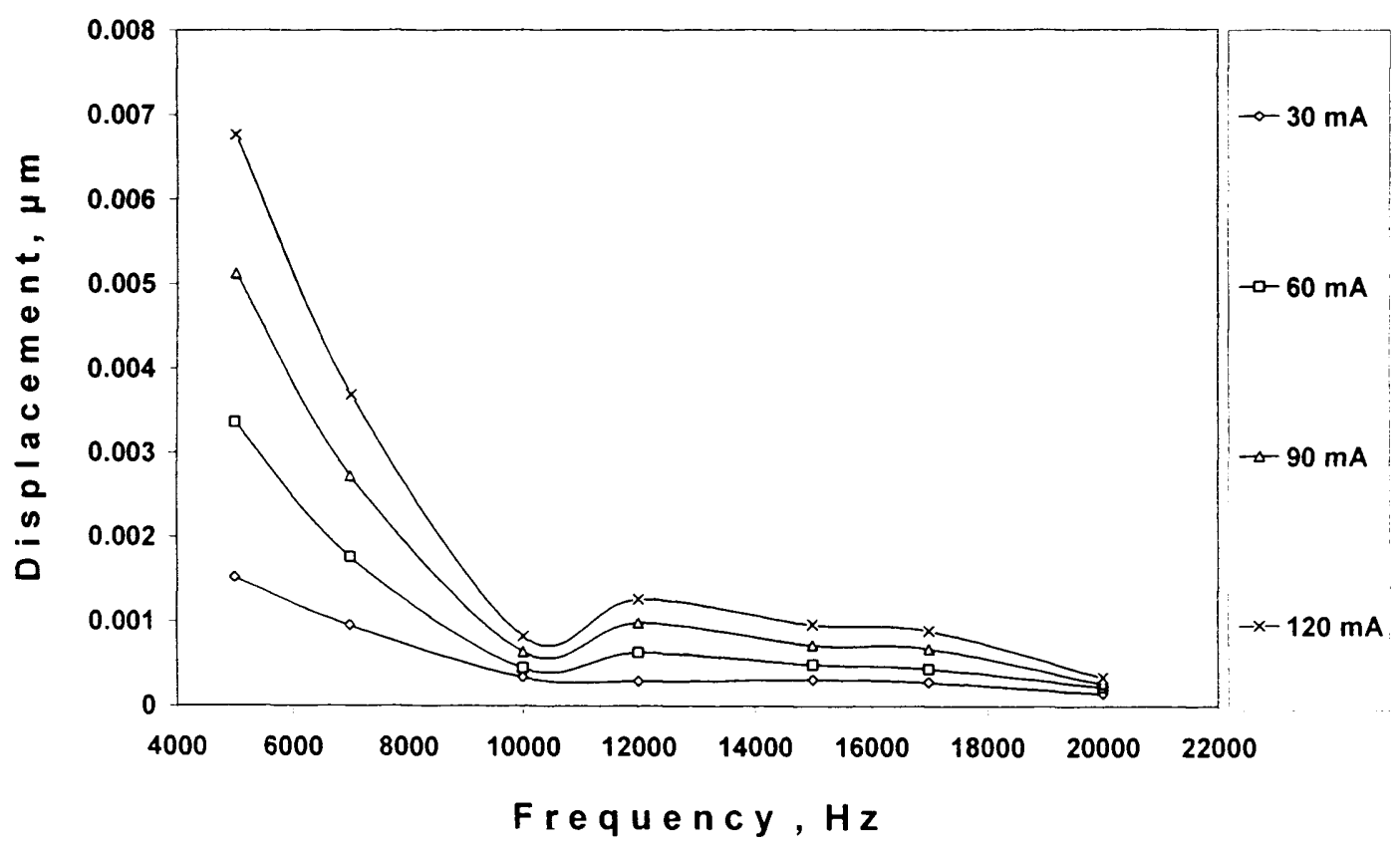

Figure 7.36: Displacement profile (non-resonant) of the moving coil with a nonmagnetic diaphragm configuration of the transducer speaker showing displacements in the frequency range of $5 \mathrm{kHz}$ to $20 \mathrm{kHz}$ with excitation currents varying from 30 $m A$ to $120 \mathrm{~mA}$. Displacements measured with laser incident on the centre of the vibrating diaphragm. 


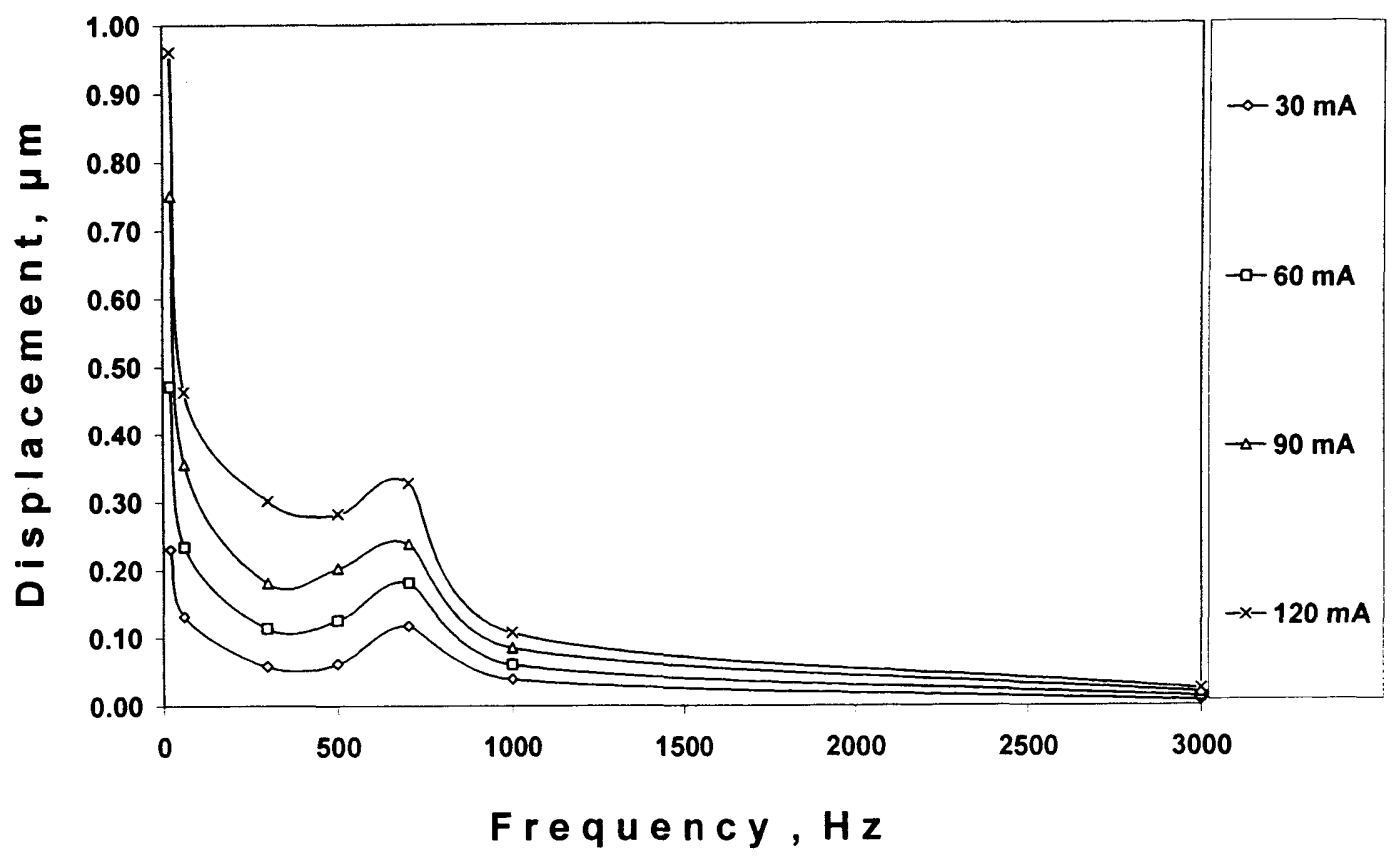

Figure 7.37: Displacement profile (non-resonant) of the moving coil with a nonmagnetic diaphragm configuration of the transducer speaker showing displacements in the frequency range of $20 \mathrm{~Hz}$ to $3 \mathrm{kHz}$ with excitation currents varying from 30 $m A$ to $120 \mathrm{~mA}$. Displacements measured with laser incident on the centre of the vibrating diaphragm.

The displacement versus frequency responses (Figs. 7.36 and 7.37) for the moving coil with a non-magnetic diaphragm combination show that this configuration produced very low displacements over the whole audible frequency range. The displacements were at least ten percent lower than that of the moving coil configuration. The absence of a flux closure path, which also acted as the panel for the transducer speaker, resulted in lower displacements for this particular arrangement. In the low frequency region, especially below $1 \mathrm{kHz}$, unstable responses were observed for this transducer configuration. The reason for such unstable behaviours at the low end of the audio frequency spectrum was due to the lack of a proper return mechanism for the vibrating panel. In a moving magnet or a moving coil configuration, the top and the bottom amorphous layers attract each others and provide a holding force for the vibrating diaphragm. Since in this transducer configuration, in which the magnetic upper panel was replaced with a non-magnetic one, there was no holding force or return mechanism for the moving panel, the 
miniature transducer speaker was displaying unstable behaviour at the low end of the audio frequencies where the excursion levels are high.

Therefore, it can be concluded that the use of a non-magnetic material as the base layer or as a panel for the speaker introduces instability and results in inefficient frequency responses for the transducer speaker. Both the magnetic layers provide flux closure paths and most importantly provide a magneto-mechanical return mechanism for the vibrating panel which is vital for stability in a loudspeaker system.

\subsection{Harmonic Distortions}

The total harmonic distortion, or THD, of a signal is a measurement of the harmonic distortion present [3]. When a signal passes through a non-linear device, additional content is added at the harmonics of the original frequencies. This is a measurement of the extent of that distortion.

The measurement is most commonly the square root of the ratio of the sum of the powers of all harmonic frequencies above the fundamental frequency to the power of the fundamental:

$$
T H D=\sqrt{\frac{\text { Power in Distortion }}{\text { Power in Fundamental }}}=\sqrt{\frac{\sum \text { harmonic powers }}{\text { fundamental frequency power }}}
$$

Other calculations for amplitudes, voltages and currents are equivalent. For a voltage signal, for instance, the ratio of RMS voltages is equivalent to the power ratio [4]:

$T H D=\frac{\sqrt{V_{2}^{2}+V_{3}^{2}+\ldots \ldots \ldots+V_{n}^{2}}}{V_{1}}$

In this calculation, $V_{n}$ means the RMS voltage of harmonic $n$. 
Figures 7.38 and 7.39 show the trend in harmonic distortions for the moving magnet configuration of the transducer speaker at low and high frequencies respectively. The results show the variation in harmonic distortions at various excitation currents.

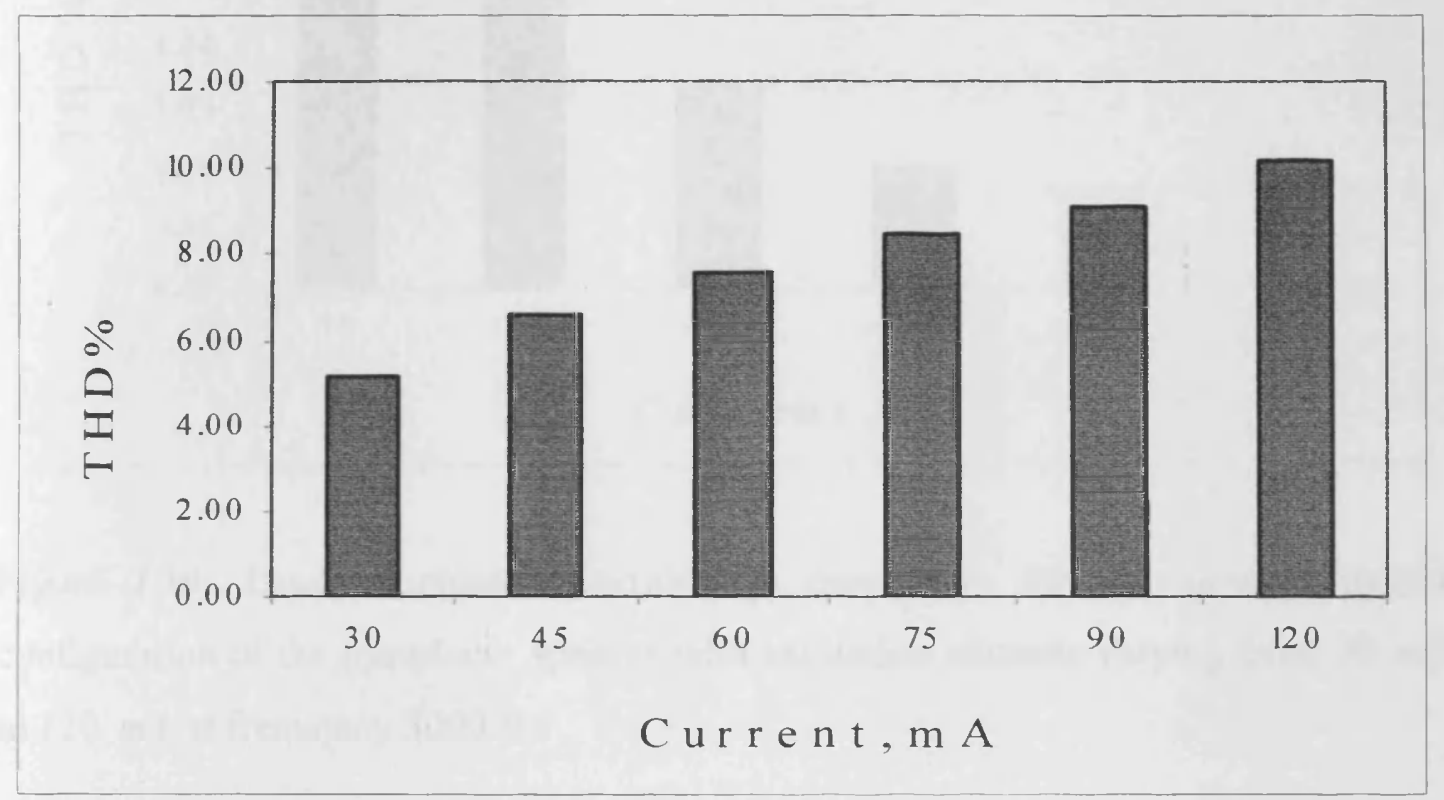

Figure 7.38: Total Harmonic Distortion in percentage for the moving magnet configuration of the transducer speaker with excitation currents varying from $30 \mathrm{~mA}$ to $120 \mathrm{~mA}$ at frequency $100 \mathrm{~Hz}$ 


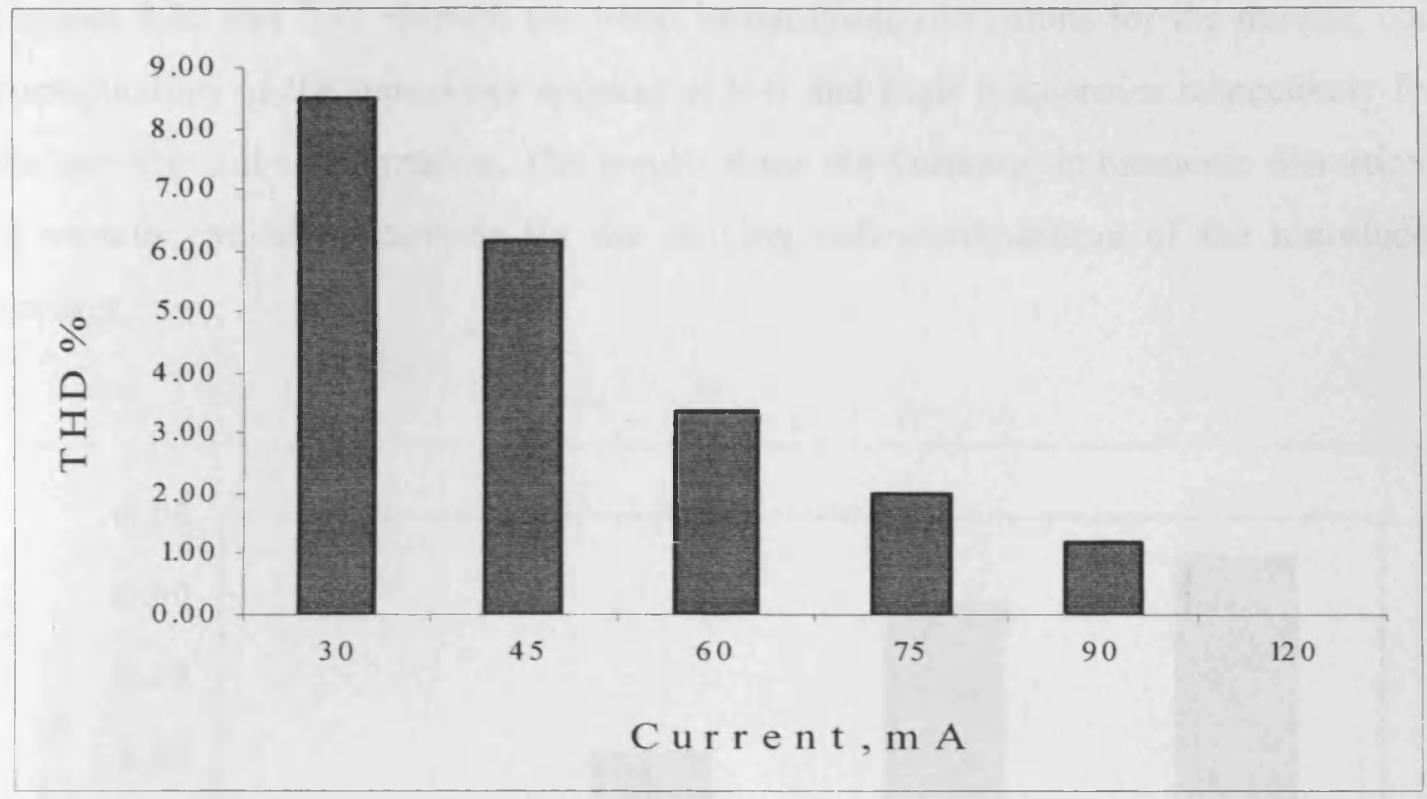

Figure 7.39: Total Harmonic Distortion in percentage for the moving magnet configuration of the transducer speaker with excitation currents varying from $30 \mathrm{~mA}$ to $120 \mathrm{~mA}$ at frequency $3000 \mathrm{~Hz}$ 
Figures 7.40 and 7.41 showed the trend in harmonic distortions for the moving coil configuration of the transducer speaker at low and high frequencies respectively for the moving coil configuration. The results show the variation in harmonic distortions at various excitation currents for the moving coil configuration of the transducer speaker.

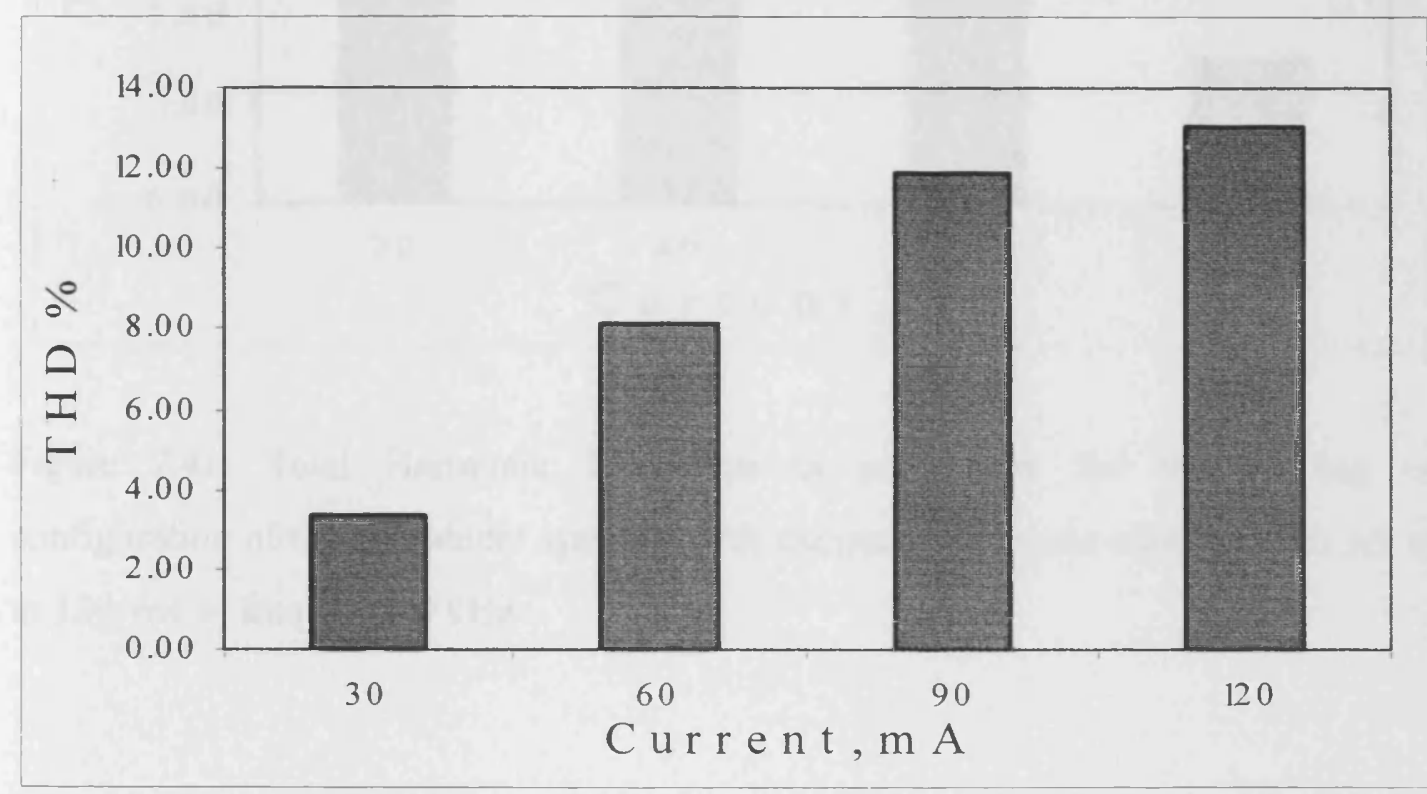

Figure 7.40: Total Harmonic Distortion in percentage for the moving coil configuration of the transducer speaker with excitation currents varying from $30 \mathrm{~mA}$ to $120 \mathrm{~mA}$ at frequency $100 \mathrm{~Hz}$ 


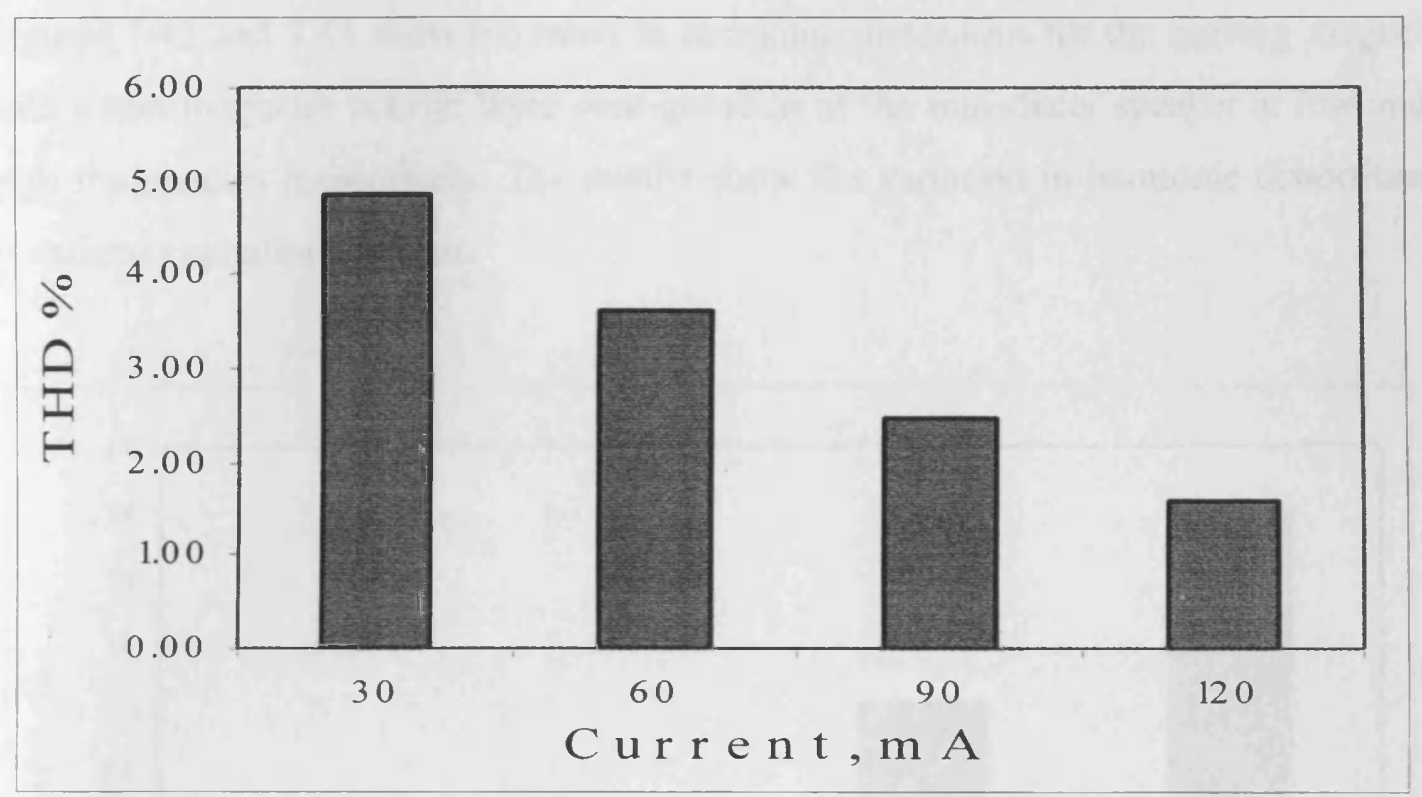

Figure 7.41: Total Harmonic Distortion in percentage for the moving coil configuration of the transducer speaker with excitation currents varying from $30 \mathrm{~mA}$ to $120 \mathrm{~mA}$ at frequency $3 \mathrm{kHz}$ 
Figures 7.42 and 7.43 show the trend in harmonic distortions for the moving magnet with a non-magnetic bottom layer configuration of the transducer speaker at low and high frequencies respectively. The results show the variation in harmonic distortions at various excitation currents.

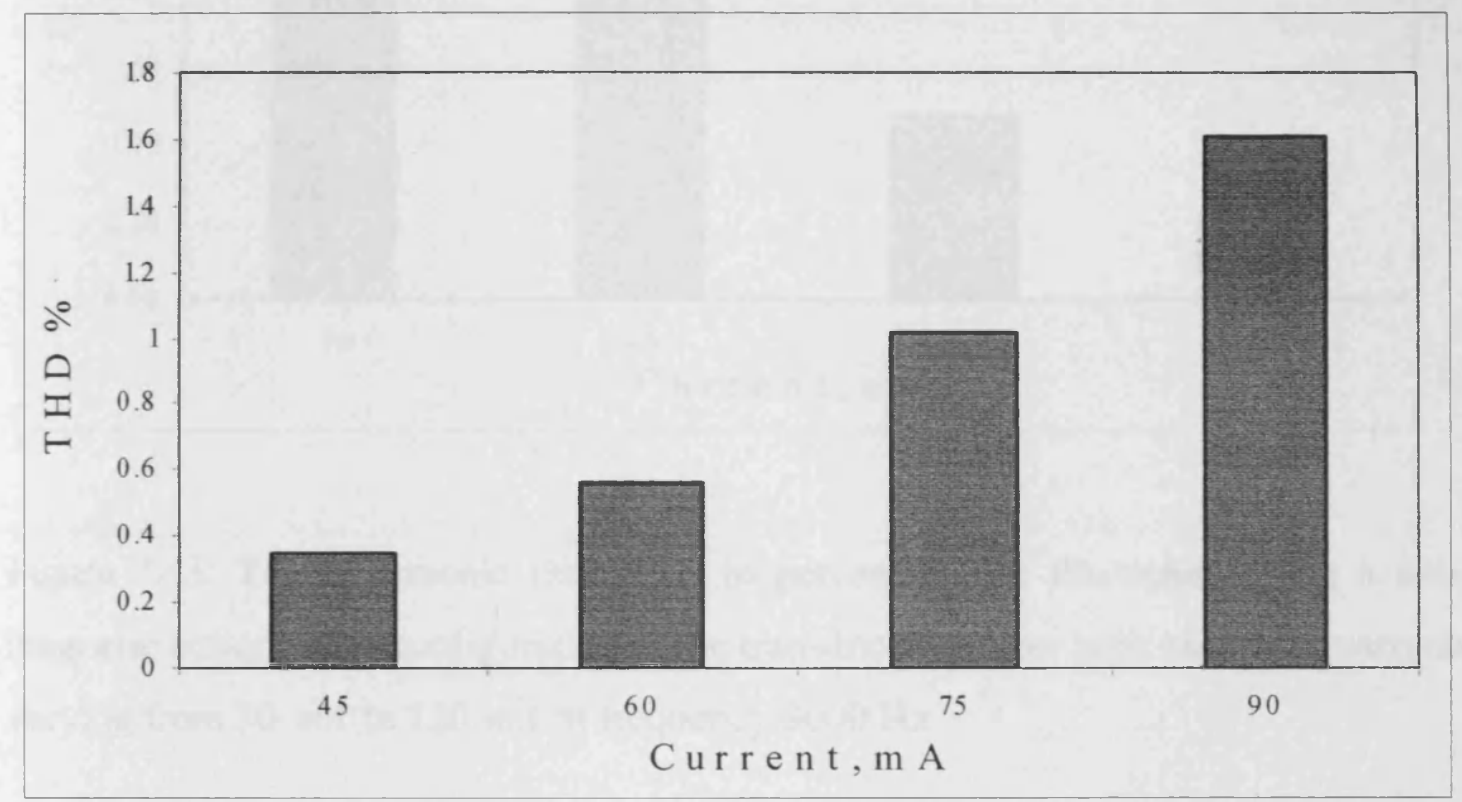

Figure 7.42: Total Harmonic Distortion in percentage for the moving magnet with a non-magnetic bottom layer configuration of the transducer speaker with excitation currents varying from $30 \mathrm{~mA}$ to $120 \mathrm{~mA}$ at frequency $100 \mathrm{~Hz}$ 


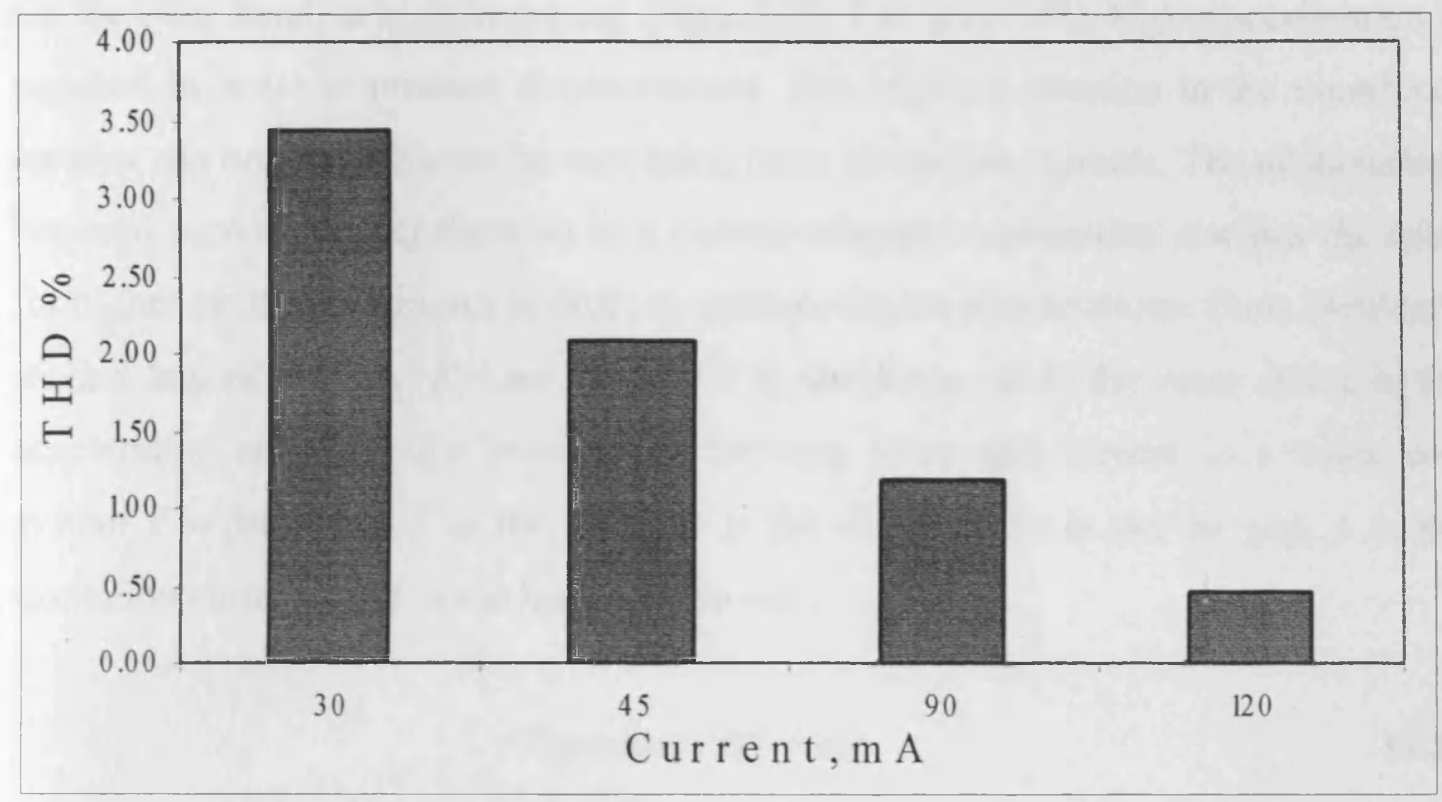

Figure 7.43: Total Harmonic Distortion in percentage for the magnet with a nonmagnetic bottom layer configuration of the transducer speaker with excitation currents varying from $30 \mathrm{~mA}$ to $120 \mathrm{~mA}$ at frequency $3000 \mathrm{~Hz}$

The harmonic distortion results showed the amount of distortions present in the reproduced sound. The results from the three different device configurations (Figs. $7.38,7.39,7.40,7.41,7.42$, and 7.43 ) showed that the trend in harmonic distortions changed from low to high frequency. The single cell speaker showed two distinctive trends depending on the frequency range. At low frequency range, the total harmonic distortion increased with current but at high frequency, the total harmonic distortion decreased with the level of excitation current.

At low frequency region (Figs. 7.38, 7.40 and 7.42), the harmonic distortion increased with current level in each case. The small size of the miniature transducer diaphragm served as a limitation for the low frequency sound reproduction. Since the sound wavelengths are greater at low frequency, the displacement of the diaphragm is also higher. Therefore at a certain higher excitation current the displacement of the diaphragm reaches its maximum and injecting more current results in higher harmonic distortion. 
On the other hand, at high frequency (Figs. 7.39, 7.41 and 7.43), higher acceleration is required in order to produce displacements. The high acceleration in the transducer speaker can only be achieved by supplying more excitation currents. The relationships between current and acceleration in a current-magnet combination clarifies the need for higher excitation currents in order to produce higher accelerations. From Newton's second law of motion, $F=m a$ where $\mathrm{F}$ is the force, $\mathrm{m}$ is the mass and $\mathrm{a}$ is the acceleration and from the relationship between force and current in a voice coil system, $F=B i l$ where $\mathrm{F}$ is the force, $\mathrm{B}$ is the flux density in the air gap, $i$ is the excitation current and $l$ is the length of the coil.

Therefore, $B i l=m a$

Since $B, l$ and $m$ in this device can be assumed to be constant, therefore the following relation can be deduced for the transducer speaker:

$$
i \alpha \quad a
$$

Therefore, since the acceleration in this system is proportional to the excitation current, in order to get high acceleration from the panel more current is required.

The magnitude of displacement decreases with frequency as we have observed from the displacement results for the transducer speakers. From equation 7.4 in section 7.2.1, it can be deduced that an increase in the magnitude of displacement at high frequencies is possible only if the acceleration is increased.

At high frequencies, the magnitude of loudspeaker displacement is very small and as a result the output displacement from the speakers is easily affected by the surrounding noise. However, as the acceleration or the magnitude of displacement is increased by providing more excitation current, the noise becomes less significant. Therefore at high frequencies, harmonic distortion reduces with the increase in excitation currents. 


\subsection{Effects of Neighbouring Transducers in an Array}

Two magnets repel each other when placed alongside and this repelling force determines the minimum distance between them. Finite element modeling has been used to determine the force profile and the minimum distance between the transducers in the matrix array loudspeaker (Fig. 7.44). The closest proximity achievable between any two transducers within the matrix array was found to be $0.5 \mathrm{~mm}$.

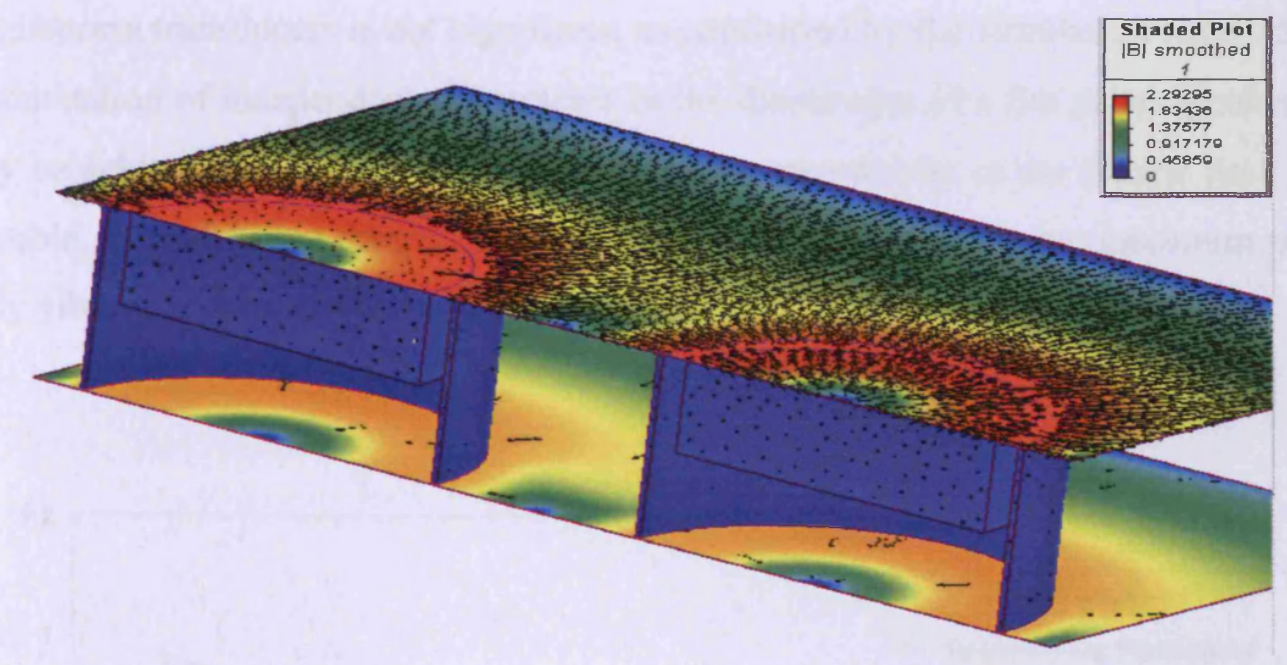

Figure 7.44: Simulation showing interaction between neighbouring transducers in the matrix array

A loudspeaker was constructed using two novel transducers separated by the minimum distance, as found by the simulation. The reason for placing two transducers next to each other at a minimum distance was to observe the effect of their interactions on the displacement characteristics. Both the transducers, connected in series and coupled by the same membrane, were excited by an excitation current of $100 \mathrm{~mA}$. The following graphs showed the effect of placing two transducers next to each other at a distance of 0.5 millimetres. The effect of the interactions between transducers can be observed by the variation in their displacement characteristics. Figure 7.45 showed the comparative displacement characteristics of an isolated and a neighbouring transducer over the whole audio frequency range. Figures 7.46 and 7.47 show the comparative graphs in detail in the low and high frequencies respectively. 
The results (Figs. 7.45, 7.46 and 7.47) showed that the displacement profile of a neighbouring transducer matches closely to that of an isolated single transducer. The influence of the mechanical coupling is not significant since similar results have been obtained by placing alongside two separate transducers that are not coupled by a continuous membrane. Therefore electromagnetic transducers separated by a minimum distance have insignificant effect on their individual displacement characteristics as evident from the experimental results.

At this 'minimum distance', the interaction between the flux lines produced by the neighboring transducers is not significant as confirmed by the simulation result. Since minimization of independent movements in the diaphragm of a flat panel speaker can only be achieved by placing numerous miniature transducers in the closest proximity possible, this minimum distance between the transducers will ensure maximum whole body vibration.

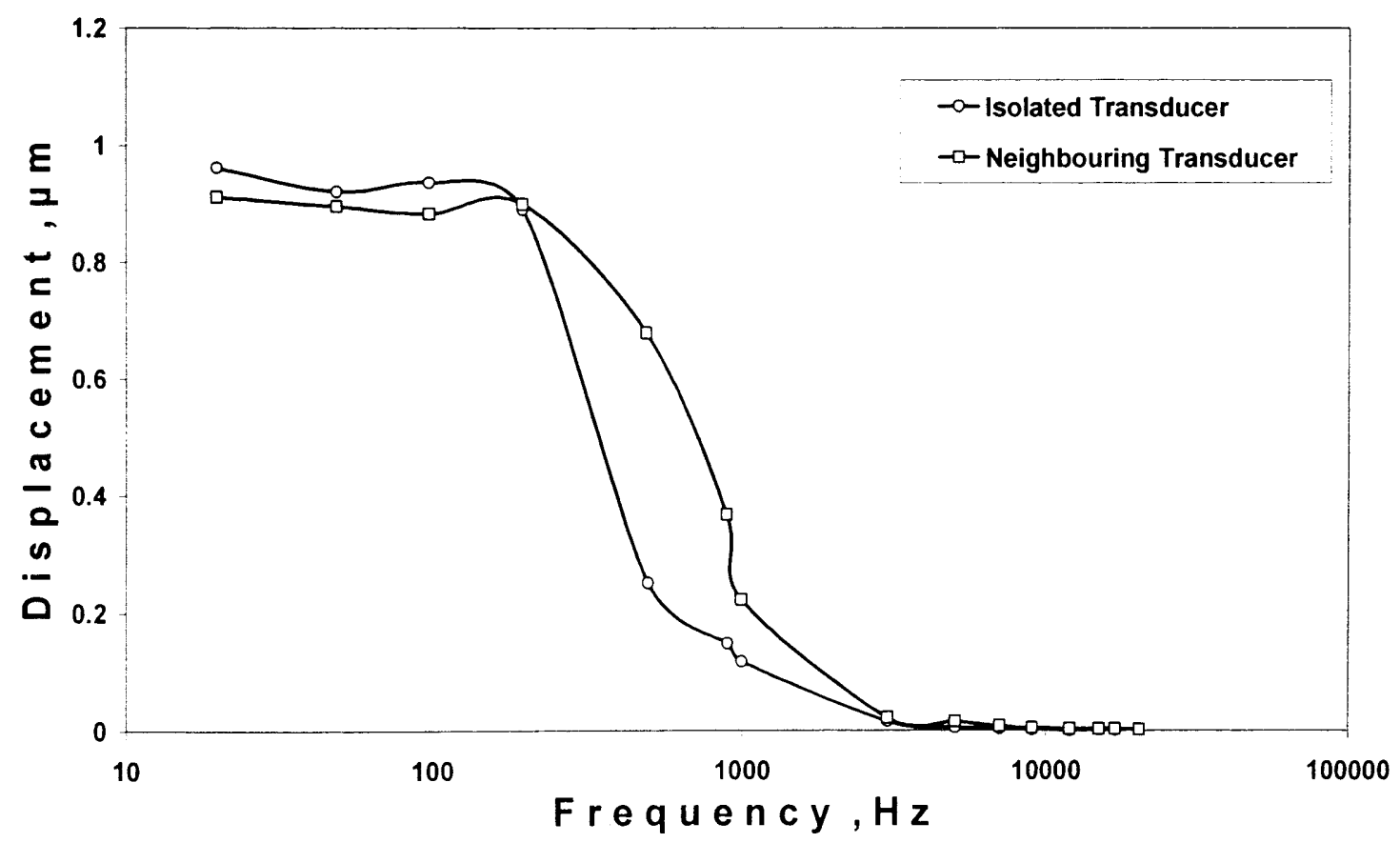

Figure 7.45: Displacement characteristics for an isolated and a neighbouring transducer for the audible frequency range $(20 \mathrm{~Hz}$ to $20 \mathrm{kHz})$. Neighbouring transducers were separated at a distance of $0.5 \mathrm{~mm}$ and the measurements were taken at an excitation current of $100 \mathrm{~mA}$. 


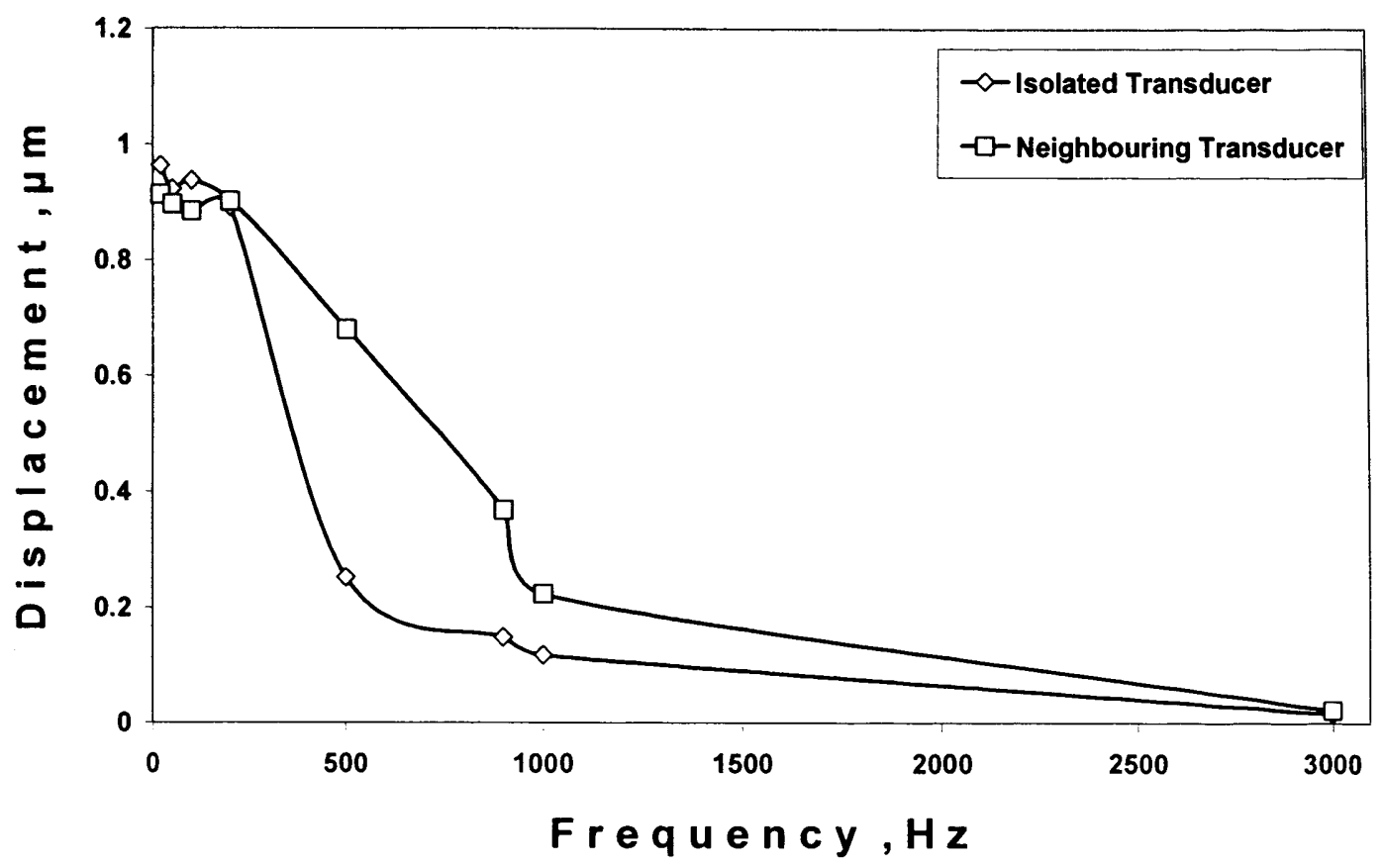

Figure 7.46: Displacement characteristics for an isolated and a neighbouring transducer in the low frequency range $(20 \mathrm{~Hz}$ to $3 \mathrm{kHz})$. Neighbouring transducers were separated at a distance of $0.5 \mathrm{~mm}$ and the measurements were taken at an excitation current of $100 \mathrm{~mA}$

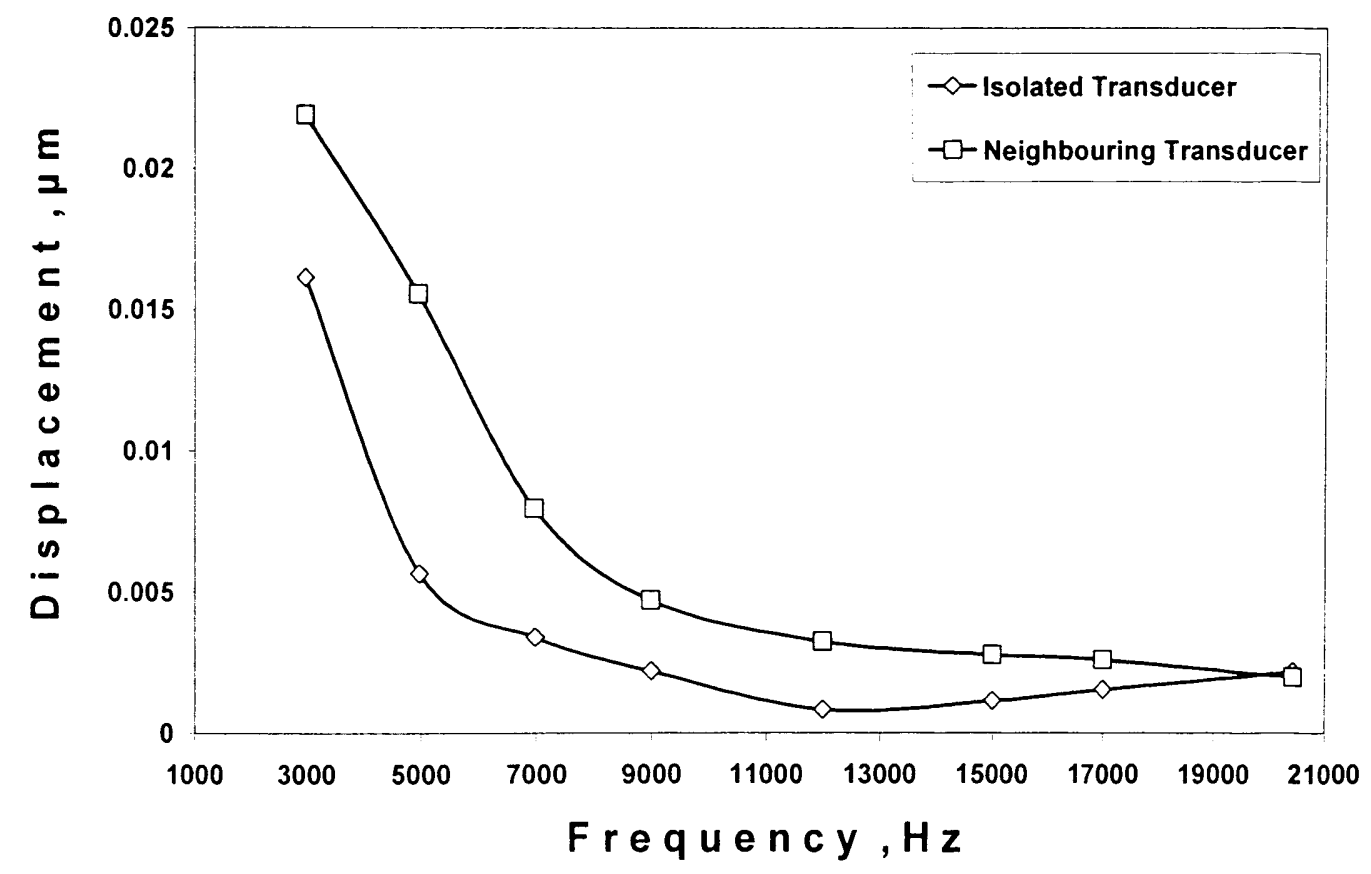

Figure 7.47: Displacement characteristics for an isolated and a neighbouring transducer in the high frequency range $(3 \mathrm{kHz}$ to $20 \mathrm{kHz})$. Neighbouring transducers were separated at a distance of $0.5 \mathrm{~mm}$ and the measurements were taken at an excitation current of $100 \mathrm{~mA}$ 


\subsection{Displacement Profile of the Vibrating Panel}

The novel transducer speaker was tested for displacement profile of the diaphragm using the laser vibrometer pointing at different points of the vibrating surface. Three different points on the vibrating panel were chosen to carry out the experiment. The first point was at the middle point or at the centre of the axis on the panel, the second point was at $1.6 \mathrm{~mm}$ off the centre point and the third point on the panel was at $3 \mathrm{~mm}$ off the centre of the axis on the vibrating panel. In each case the excitation current was $100 \mathrm{~mA}$. Figure 7.48 and 7.49 show the displacement characteristics of the three different points at low and high frequencies respectively.

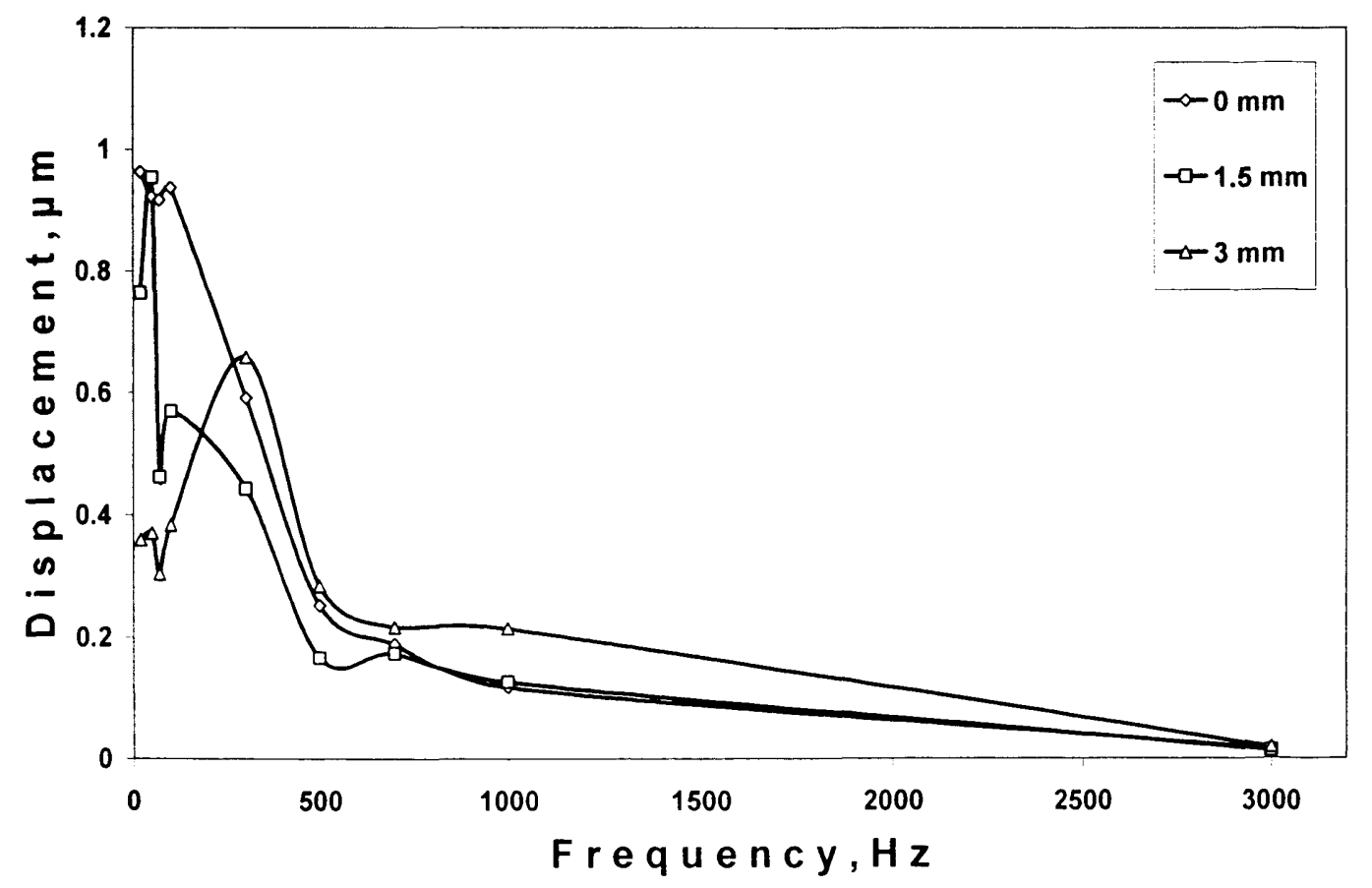

Figure 7.48: Displacement profile for the vibrating panel in the frequency range $20 \mathrm{~Hz}$ to $3 \mathrm{kHz}$ showing the variation in displacements measured at three different points on the diaphragm. The three measured points are: centre of the vibrating panel $(0 \mathrm{~mm})$, $1.5 \mathrm{~mm}$ off the centre point and $3 \mathrm{~mm}$ off the centre point. Excitation current is 100 $m A$ 


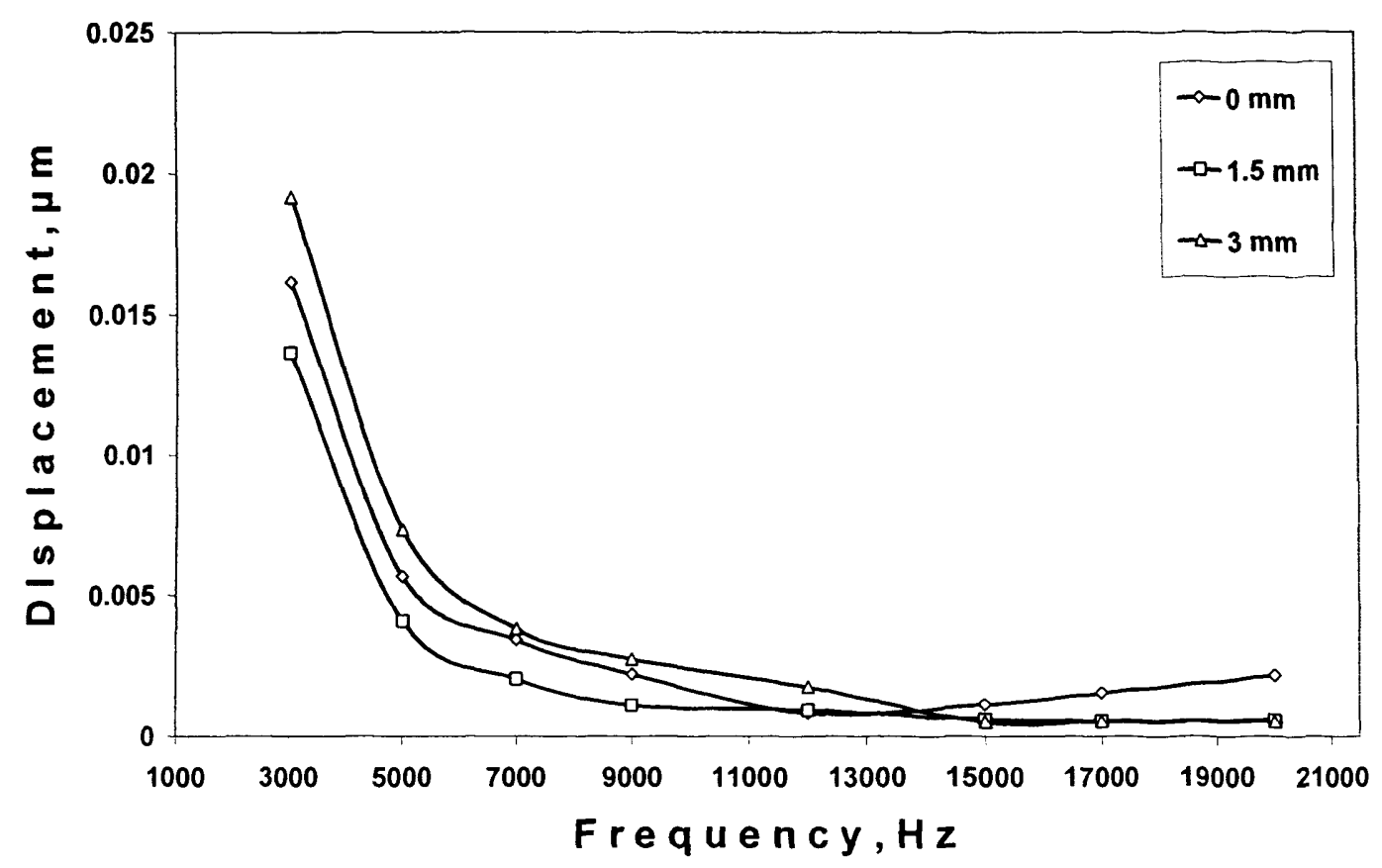

Figure 7.49: Displacement profile for the vibrating panel in the frequency range $3 \mathrm{kHz}$ to $20 \mathrm{kHz}$ showing the variation in displacements measured at three different points on the diaphragm. The three measured points are: centre of the vibrating panel $(0$ $\mathrm{mm}), 1.5 \mathrm{~mm}$ off the centre point and $3 \mathrm{~mm}$ off the centre point. Excitation current is $100 \mathrm{~mA}$.

The results (Figs. 7.48 and 7.49) from the displacement measurements on these points of the vibrating panel showed that displacement was maximum at the centre of the vibrating panel and it decreases as the point of vibration shifts away from the centre. It was observed from the displacement profile of these three points that in the low frequency region (Fig. 7.48), the vibrating points that were away from the centre showed unstable behaviour. This is due to the fact that the vibrating panel itself was not mechanically attached to the rest of the speaker structure and hence the higher excursions at the low frequency region created unstable responses especially around the edges of the coil in the transducer speaker. However, in the high frequency region (Fig. 7.49), the difference in the displacement profile among these points is insignificant because of the very low excursion levels of the loudspeaker diaphragm at high frequencies. 


\subsection{Pressure Response Curves from Transducers Arrays}

Pressure responses from a $2 \times 2$ array and a 9x2 array matrix speaker have been measured using a high bandwidth microphone and the results are presented in figure 7.45. Both speakers were driven with the same excitation current. The following equation [5] was used to obtain the measured pressure responses in decibel.

$$
\text { Sound Pr essure Level }(S P L), d B=20 \log _{10}\left(\frac{P}{P_{0}}\right)
$$

In the above equation, $P$ is the sound pressure being measured and $P_{0}$ is the reference sound air pressure which is $20 \mu \mathrm{Pa}$.

Miniature moving coil transducers have been used to construct a $2 \times 2$ array and a $9 \times 2$ matrix array speaker. The sound pressure levels measured from these matrix array speakers show the bandwidth of these speakers in terms of reproducing sound in the audible frequency range. The sound pressure levels have been measured using a high bandwidth microphone with $100 m A$ excitation current. 


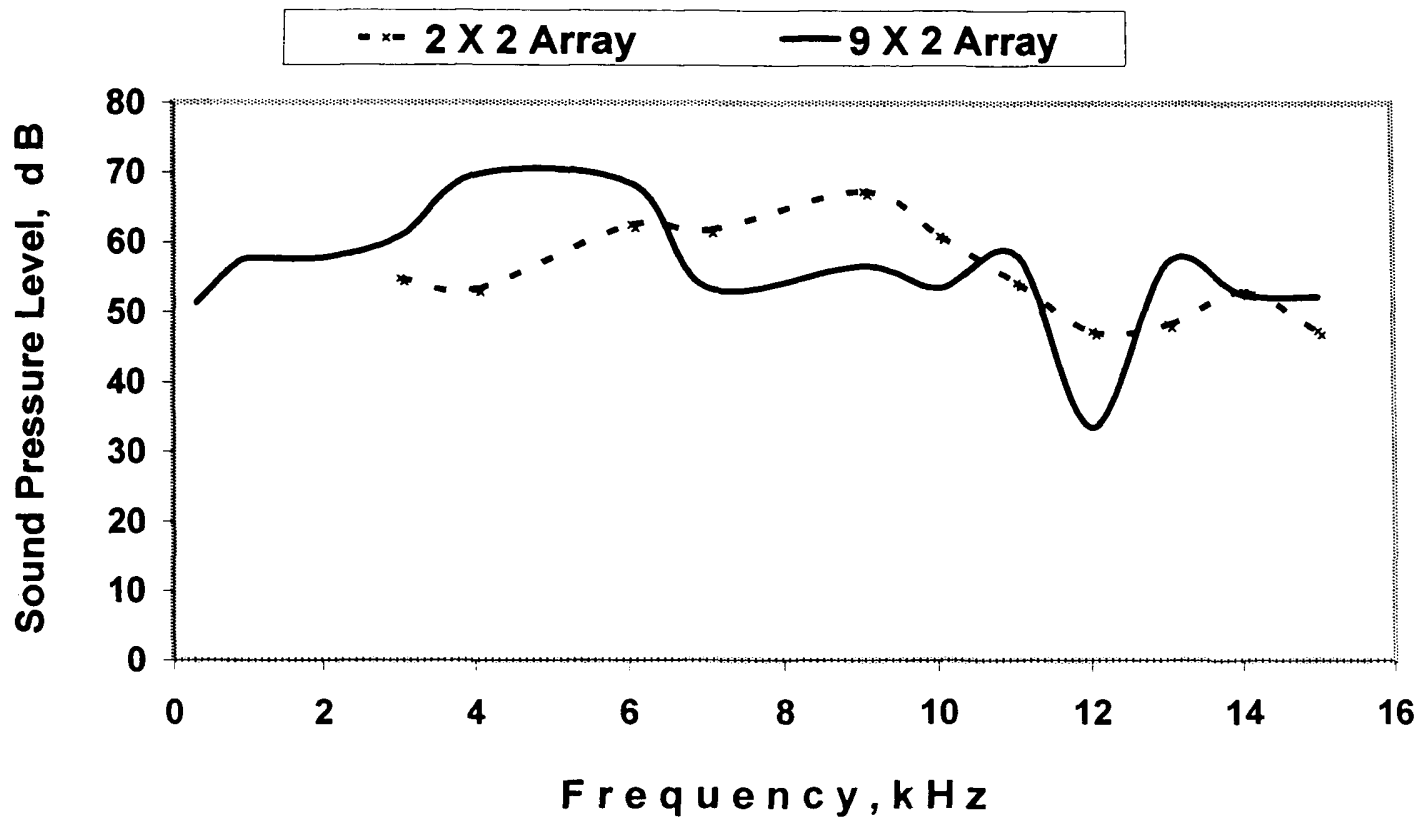

Figure 7.50: Sound pressure levels for the $2 \times 2$ and $9 \times 2$ matrix array speakers in the audio frequency range $(20 \mathrm{~Hz}$ to $20 \mathrm{kHz})$ showing improvement in bass response for the longer array speaker. Sound pressure measurements were taken using a high bandwidth microphone positioned perpendicularly to the vibrating panel at a metre distance. Excitation current was $100 \mathrm{~mA}$.

The physical width of the available amorphous ribbon used in this project restricted the width of the matrix array transducer speakers. The width of the matrix array transducer speaker could only accommodate two miniature transducers and hence a $2 \times 2$ and a 9x2 matrix array speaker were constructed using the miniature transducers. For the $2 \times 2$ array speaker, the lowest frequency observed was $3 \mathrm{kHz}$ and below this frequency the sound pressure was too low to be measured by a high bandwidth microphone. For the $9 \times 2$ array speaker, however, the lowest frequency at which sound pressure could be measured was around $50 \mathrm{~Hz}$. The 9x2 array speaker has better acoustic pressure response in the bass region compared to the $2 \times 2$ array speaker at the same distance. The results from the pressure response measurement show the improvement in bass response for the $9 \times 2$ array speaker. A larger surface area and the higher force obtained by employing more active transducers resulted in the improvement of the bass response for the longer array speaker. 
The 9x2 array speaker was impedance matched at $8 \Omega$ for standard amplifiers whereas the impedance of the $2 \times 2$ array was of $0.7 \Omega$ because of its parallel connection. Because the panel of the 9x2 array speaker has a larger surface area and has more actuators attached to it than the $2 \times 2$ array speaker, it was able to drive more air mass in front of it. This result from the transducer array speakers shows that the matrix configuration of miniature transducers improves the low frequency response. The efficiency and sensitivity of miniature loudspeakers has been improved by means of loudspeakers with a large diaphragm area, high flux density, a high number of turns, a small voice coil resistance and small mass. The large diaphragm in a larger array speaker has extended the low frequency operation down to $50 \mathrm{~Hz}$. 


\subsection{Impedance, Resistance and Inductance Curves}

The impedance characteristics of the novel miniature transducer speaker have been observed by using an impedance analyzer and also the test results. Figure 7.51, 7.52 and 7.53 show the impedance, resistance and inductance profile of the transducer respectively at audio frequencies measured by an impedance analyzer. Figure 7.54 shows the impedance profile of the miniature transducer speaker as calculated from the variation in the supply voltage as the frequency was increased in the loudspeaker displacement measurement.

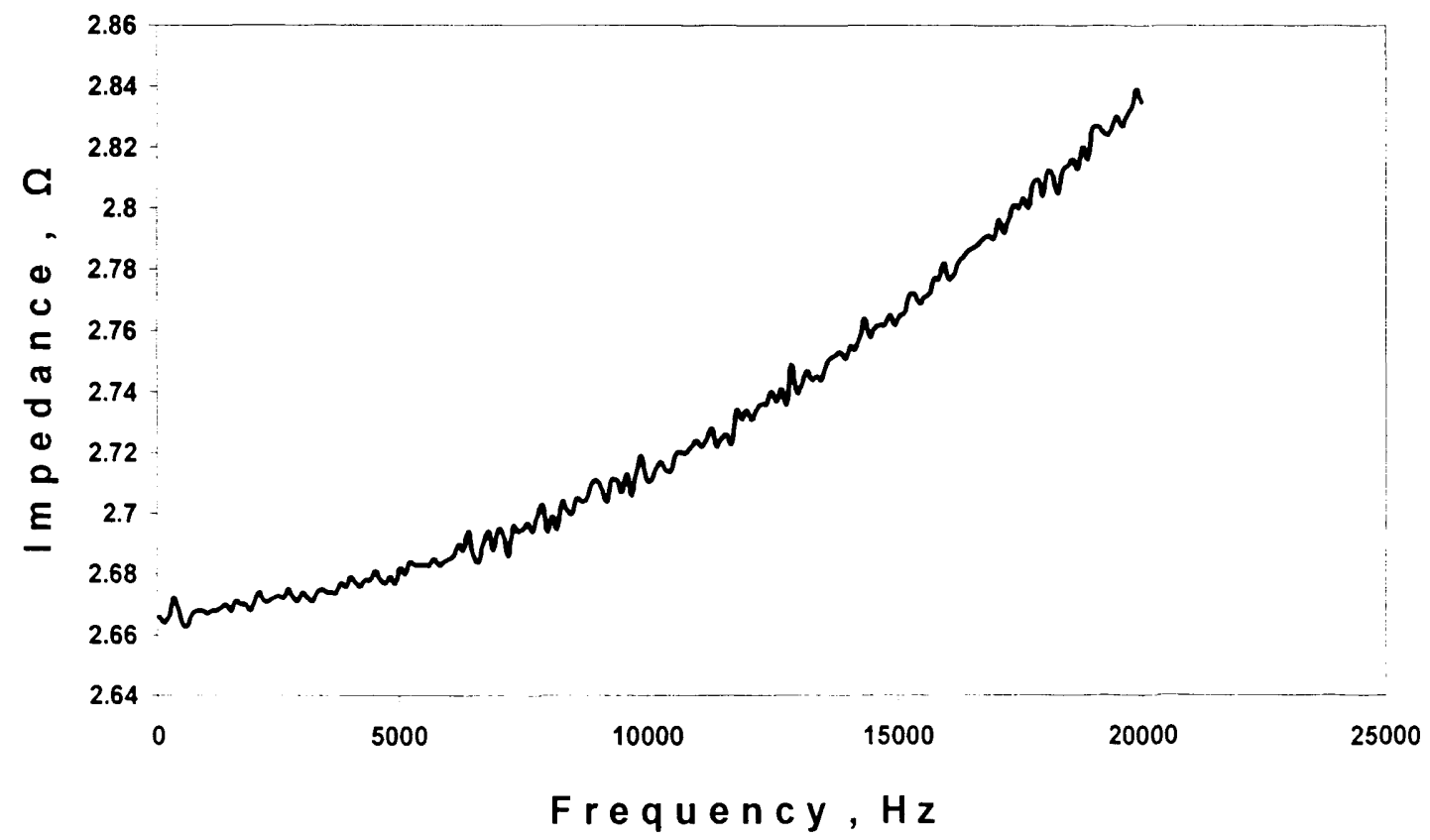

Figure 7.51: Impedance profile of a miniature transducer speaker in the audio frequency range $(20 \mathrm{~Hz}$ to $20 \mathrm{kHz})$ as measured by an Impedance Analyzer. 


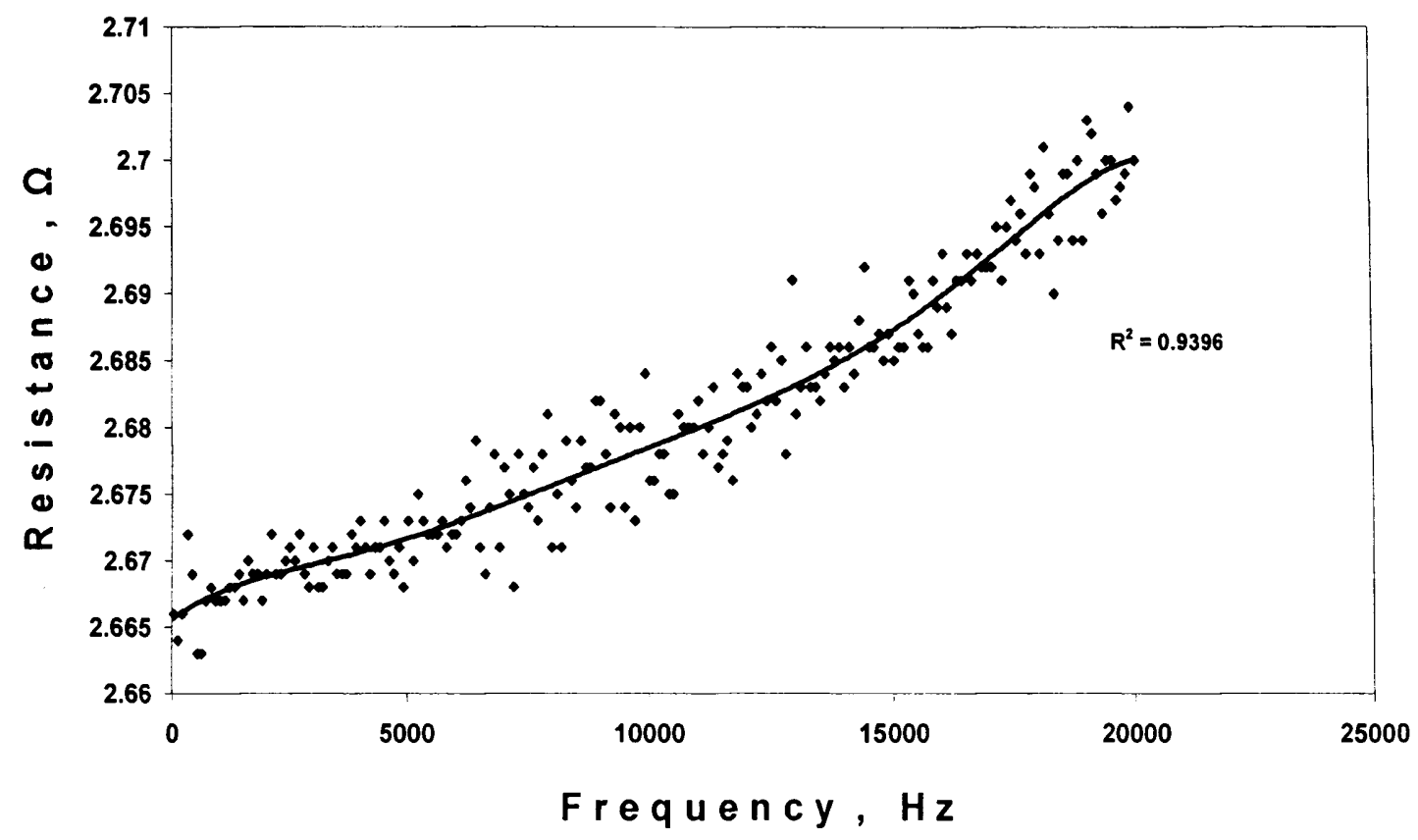

Figure 7.52: Resistance profile of a miniature transducer speaker in the audio frequency range $(20 \mathrm{~Hz}$ to $20 \mathrm{kHz})$ as measured by an Impedance analyzer. A confidence value of 0.9396 was obtained for the curve drawn from measured data points.

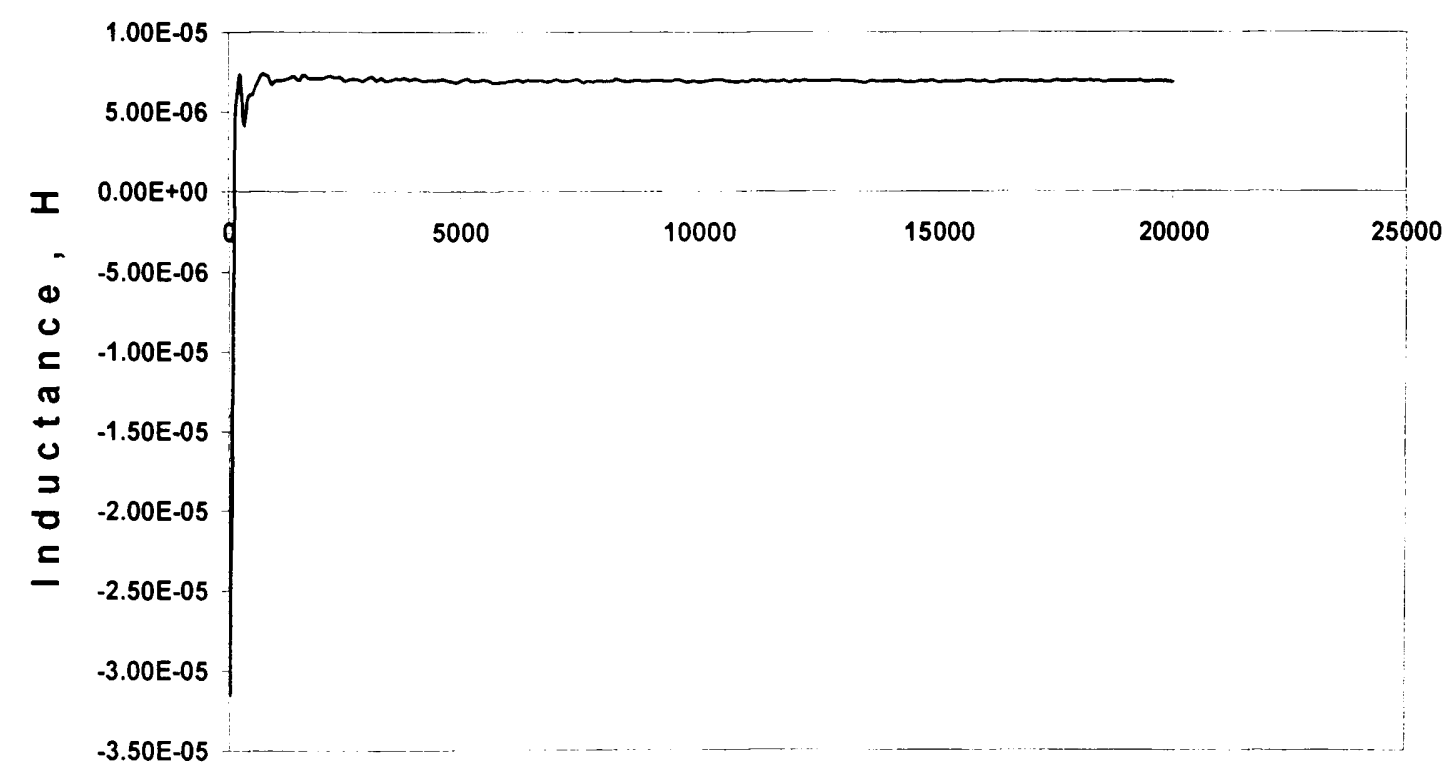

Frequency, Hz

Figure 7.53: Inductance profile of a miniature transducer speaker in the audio frequency range ( $20 \mathrm{~Hz}$ to $20 \mathrm{kHz})$ as measured by an Impedance analyzer 


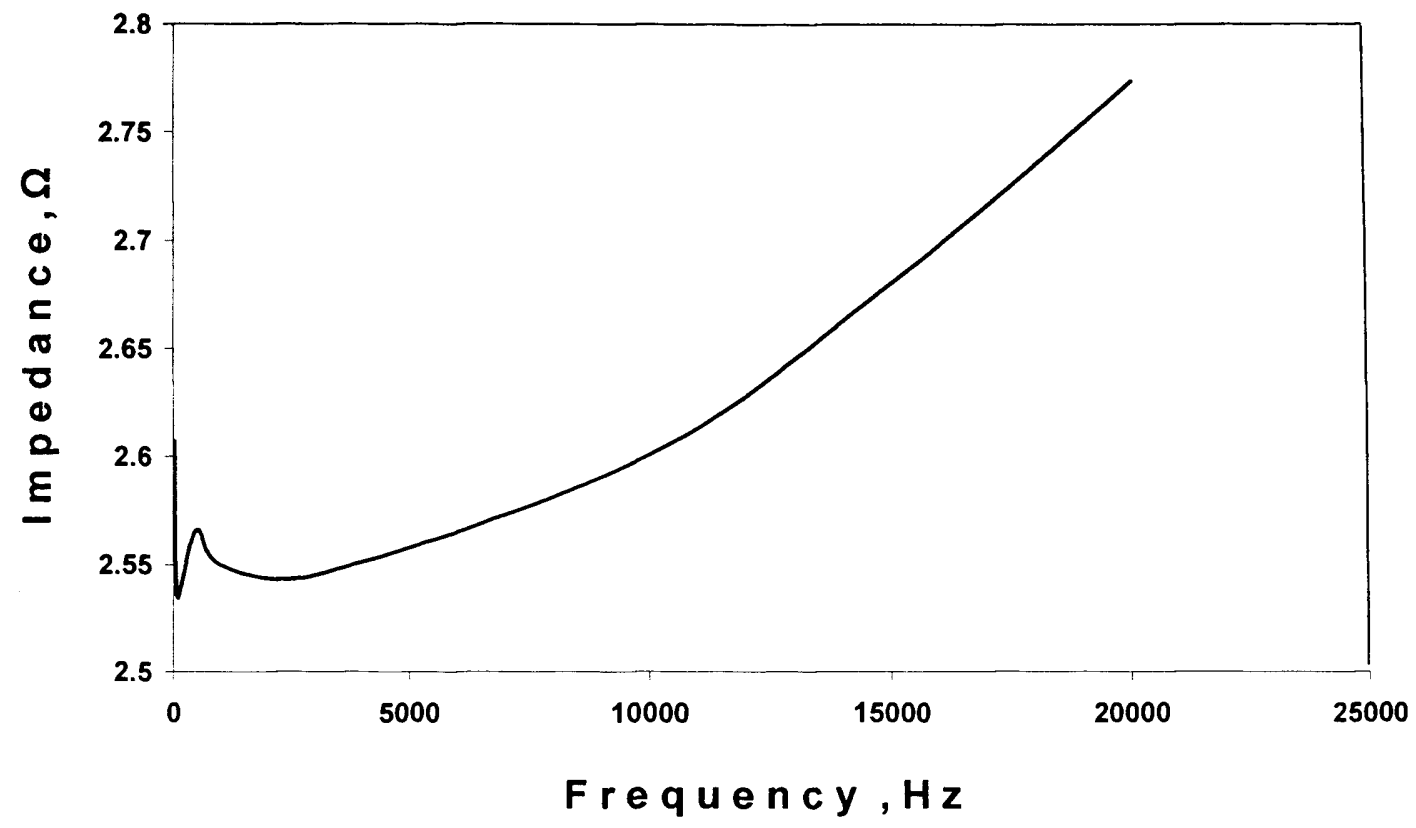

Figure 7.54: Impedance profile of a miniature transducer speaker in the audio frequency range $(20 \mathrm{~Hz}$ to $20 \mathrm{kHz})$ as calculated from the displacement measurements.

Impedance measurements of a single transducer (Figs. 7.51, 7.52, 7.53 and 7.54) showed an increase in impedance as the frequency changed from $20 \mathrm{~Hz}$ to $20 \mathrm{kHz}$. The impedance increased by only $7 \%$ over the whole audible frequency range and hence had no significant effect on the performance of the loudspeaker. The inductance remained constant and, since the value of inductance was very low compared to the resistance of the coil, the reactance which increased with the frequency did not have a significant effect on the overall impedance. 


\section{References:}

[1] Image of the Threshold Sensitivity Curve for the Human Ear http://hyperphysics.phy-astr.gsu.edu/hbase/sound/earcrv.html\#c2

[2] F. W. Sears, M. W. Zemansky, H. D. Young, "University Physics," Narosa Publishing House, $17^{\text {th }}$ Reprint, 1998

[3] F. C. D. L. Rosa, "Harmonics and Power Systems," CRC Press Inc, $1^{\text {st }}$ Edition, 2006

[4] D. Shmilovitz, "On the Definition of Total Harmonic Distortion and Its Effect on Measurement Interpretation," IEEE Transactions on Power Delivery, Vol. 20, Issue 1, pp. 526- 528 (2005)

[5] A. P. G. Peterson, E. E. Gross, "Handbook of Noise Measurement," General Radio Co., $2^{\text {nd }}$ Edition, 1972 
The idea of designing a novel loudspeaker is based on the current limitations of the flat panel speakers. The present flat panel loudspeakers exhibit poor low frequency and poor impulse response. In order to reduce these limitations, the innovative approach of introducing conventional whole body vibration mechanism to flat panels has been developed. Based on this novel approach, a miniature transducer speaker has been designed and developed. This new miniature transducer speaker aims to provide a solution to the miniaturization of loudspeaker with flat panel speaker technology, which operates under bending wave principle, and exploits the resonance of the panel instead of the traditional piston motion to produce sound.

In the early stage of the design and development process, a feasibility study has been undertaken to identify the most suitable electromagnetic transduction mechanism for the novel speaker system. The feasibility study combines background research and experimental results on different commercial actuators. A comparative study on two different electromagnetic transducer combinations namely the current-magnet and the current-iron, and an analysis of the experimental results from these transducers have been presented in chapter 6 .

A solenoid transducer (current-iron) has various disadvantages which makes it unsuitable for loudspeaker application. The surrounding iron frame and the iron plunger in the solenoid transducer introduce hysteresis in the magnetic circuit; produce eddy current loss and skin effect. Moreover, the variable reluctance of the magnetic circuit produces non-linear force-current relationship which is not ideal for a loudspeaker application. The motion of the plunger in a solenoid transducer is controlled by a return spring and the resonance-associated with the spring introduces unwanted oscillation and noise in the transducer's output. 
On the other hand, as observed from practical experimentation and FEM modelling, a voice-coil transducer (current-magnet) could produce non-distorted output over the whole audible frequency range. Since a magnetic material (i.e. iron) is not the active part of the voice coil actuation process, the limitations associated with magnetic hysteresis, eddy current loss and skin effect do not affect the output of this transducer. Also a linear force versus current and a linear displacement versus current relationship for the currentmagnet transducer make it the most suitable transduction mechanism for loudspeaker application. Therefore, miniature novel electromagnetic transducers having a currentmagnet combination has been chosen to construct a matrix of transducers that can work as a miniature matrix-array loudspeaker.

A novel miniature electromagnetic transducer has been developed that can operate over the audible frequency range. The proposed transducer speaker is a permanent magnet placed on a high permeable amorphous ribbon which acts as the panel for the speaker. The other part of the transducer is a $7 \mathrm{~mm}$ diameter coil attached to a bottom layer made of another amorphous ribbon. The permanent magnet is a Neodymium Boron Iron magnet, $6 \mathrm{~mm}$ in diameter and is enclosed by a $0.12 \mathrm{~mm}$ diameter copper wire coil. The novel transducer speaker is based on the operating principle of a voice coil transducer. However, in terms of the device configuration and materials, it varies significantly from the conventional approach. Matrix array speakers, using these miniature transducers, have been constructed for experimentation and analysis.

Tests have been initially carried out on three different device configurations: the moving magnet, the moving coil and a moving magnet with a non-magnetic bottom layer in order to optimize the proposed design. Each of these configurations has been tested for the whole audible frequency range using a laser vibrometer and a high bandwidth microphone. The experimental results along with simulations have been used to optimize the novel transducer and the matrix array speaker.

For each configuration of the transducer speaker, results and analysis have been presented for the displacement characteristics, linearity issues, sound intensities at 
various frequency and excitation currents, the flatness of the acoustic intensity responses and the nature of harmonic distortions. The displacement profile of the vibrating panel, variation of impedance with frequency and the resonance characteristics have also been discussed. The matrix array structure using these novel transducers results in the improvement of loudspeaker performance for the audible frequency. The parameters that affect the transducer array speaker performance and also effects of transducer spacing on the performance of the matrix array speaker have been discussed in detail. The discussion along with the analysis of the results has helped to understand the functional behaviour of the novel miniature transducer array speaker.

The efficiency and sensitivity of miniature loudspeakers have been improved by means of moving coil transducer speakers driven at high excitation currents. Experimental results and analysis show that a moving coil configuration of the miniature transducer speaker is best suited for loudspeaker application. Compared to other configurations, the moving coil configuration provides lower mass for the vibrating panel and hence this device arrangement was able to produce a flatter acoustic intensity over a wide range of audio frequencies. The experimentation with a non-magnetic diaphragm proves that the magnetic layers are vitally important in terms of loudspeaker performance. Apart from working as a panel, a magnetic diaphragm serves as a flux closure path and provides mechanical return mechanism for the vibrating panel. The experimental results from the miniature matrix array transducer speakers show that a matrix array speaker incorporating a higher number of miniature transducers can significantly improve the low frequency response of the novel loudspeaker. 


\subsection{Conclusion}

A miniature matrix array transducer system for loudspeaker has been designed and developed for the first time using an electromagnetic approach. This novel and unique design of the miniature matrix array transducer system successfully integrates the technology behind conventional loudspeakers to that of the modern flat panel speakers. Taking into consideration the consistent frequency response, improved bass response and minimized magnetic interaction the matrix array transducers can improve the existing flat panel loudspeaker technology.

The new design of each of the electromagnetic miniature transducer pixel also introduced a radical change in the way conventional voice coil transducers are designed. Unlike any other voice coil design, in this ground-breaking novel design of the electromagnetic transducer, the magnetic amorphous ribbons have been utilized both as panels and a flux closure path. Also, in the novel loudspeaker design, the mechanical structure of the transducer is held together by the permanent magnet and the panels themselves. This unique feature of this electromagnetic transducer makes it suitable for applications requiring low cost and less space.

A moving coil configuration is the most suitable transducer configuration for the novel loudspeaker. The results showed that the moving coil configuration can provide a flatter frequency response over a broad range of the audio frequency spectrum. A moving coil configuration provides lower mass for the vibrating diaphragm and hence this arrangement could produce higher displacements at low frequencies and higher accelerations at high frequencies. Therefore, as opposed to a moving magnet configuration, a moving coil configuration can be implemented to construct transducer array speakers. 
The moving magnet with a non-magnetic bottom layer configuration showed that this combination could be unstable mechanically because of the lack of a holding force between the top and the bottom plate. Also, the experimentation with a non-magnetic diaphragm proved that the magnetic layers are vitally important in terms of loudspeaker performance. Apart from working as a panel, a magnetic diaphragm serves as a flux closure path and provides mechanical return mechanism for the vibrating panel.

The matrix configuration of miniature transducers improved the low frequency response. Experimental results showed that the large diaphragm in the larger array speaker extended the low frequency operation down to $50 \mathrm{~Hz}$. Therefore the efficiency and sensitivity of the miniature matrix array loudspeakers have been improved by means of loudspeakers having large diaphragm area, high flux density, a high number of turns, a small voice coil resistance and small mass.

In a matrix array loudspeaker, electromagnetic transducers can operate without any mutual disturbance when separated by a minimum distance. Experimental results show the correct positioning of the transducers in a matrix configuration, as observed by the simulation, reduces the effects of interferences on the magnetic transducers.

\subsection{Future Work}

The present investigation has given rise to the need for mechanical stress analysis for the magnetic panels in order to improve understanding of the mechanism affecting the frequency responses and the distortions for the novel transducer speaker system.

Selective excitation of transducers will eliminate the need for a cross-over network. Therefore it is necessary to develop a system that can selectively excite transducers at a given frequency. 
Develop a system than can introduce phase shifts among the transducers in the matrix array loudspeaker and therefore allowing the speaker to radiate sound at a chosen direction. 


\section{Appendix A}

\section{List of Publications}

1. R. Rashedin; Dr. T. Meydan, Dr. F. Borza; "Solenoid Actuator for Loudspeaker Application" Sensors and Actuators A: Physical, Vol.: 129, Issue: 1-2, pp. 220223,2006

2. R. Rashedin; Dr. T. Meydan, Dr. F. Borza; "Electromagnetic Micro-actuator array for Loudspeaker application" Sensors and Actuators A: Physical, Vol.: 129, Issue: $1-2$, pp. 118-120, 2006

3. R. Rashedin; Dr. T. Meydan; Dr. F. Borza; “A Novel Miniature Matrix Array Transducer System for Loudspeakers", IEEE Transactions on Magnetics, Vol. 42 , pp. $3467-3469,2006$

4. R. Rashedin; Dr. T. Meydan; "Analysis of a Moving Magnet Miniature Transducer Array Loudspeaker System", proceedings of the $32^{\text {nd }}$ IEEE Industrial Electronics Society Conference, pp. 3064 - 3069, 2006

5. R. Rashedin; Dr. T. Meydan; Dr. F. Borza; "Harmonic Distortion Minimisation in Miniature Matrix Array Loudspeakers", Sensor Letters, Vol.: 5, Issue: 1, pp. 241-243, 2007

6. R. Rashedin; Dr. T. Lin; Dr. T. Meydan; "Design Method of Micro-Actuator with Magnetic Alloy Iron-based Amorphous Plates for Loudspeaker", IEEE Transactions on Magnetics, Vol. 43, pp. 2707 - 2709, 2007 
Appendix B

Publications 


\title{
Solenoid actuator for loudspeaker application
}

\author{
R. Rashedin, T. Meydan* \\ Wolfson Centre for Magnetics Technology, Cardiff School of Engineering, Cardiff University, \\ Newport Road, PO Box 925, Cardiff CF24 OYF, United Kingdom \\ Received 23 September 2005 \\ Available online 18 January 2006
}

\begin{abstract}
A novel loudspeaker design has been proposed that employs hundreds of micro transducers producing small displacements within the audible frequency range. The feasibility of employing a solenoid actuator as the loudspeaker transducer has been studied and discussed in this paper. Compared to its counterpart, voice coil motor, a solenoid actuator exhibits very non-linear behaviour. The theoretical description along with practical test on a commercial solenoid actuator reveals the limitations of solenoid operation at high acceleration and current.
\end{abstract}

(C) 2005 Elsevier B.V. All rights reserved.

Keriords: Loudspeaker; Actuator; Solenoid; FEM model

\section{Introduction}

Loudspeakers are transducers that convert an electrical signal into pressure waves to produce sound. Loudspeakers can be categorised into two different types depending on their mode of operation: conventional cone type and flat panel. A typical cone type loudspeaker works by applying an electrical audio signal into the loudspeaker, from which an actuator converts the electrical signal into a mechanical motion. This conversion of energy causes the cone of the loudspeaker to move producing pressure waves, which are heard by the human ear as sound. The motion of the speaker is pistonic, in the sense that the unit moves as a whole. The cone type loudspeaker is not the perfect design solution in the reconstruction of sound as it suffers from problems. The main limitations are its physical size, its moving mass, and that higher frequencies cause the sound to become directional. The flat panel speaker operates on the principle of bending waves, where the speaker exploits resonance frequencies of the panel, thus producing random vibrations across its surface. The flat panel speaker employs an actuator, attached to its panel, that converts electrical signal into mechanical motion. The mechanical motion is in the form of random vibration of the panel that creates the non-directional sound. The major disad-

\footnotetext{
* Corresponding author. Fax: +442920876729.

E-mail address: meydan@ cardiff.ac.uk (T. Meydan).
}

vantage of the flat panel speaker is that it has severe limitation in producing low frequency sound since it employs random vibration instead of pistonic motion to produce sound. At present, the majority of the flat panel systems come with a dedicated subwoofer to counter this problem. The other problem associated with random vibration is that it produces very poor impulse response which causes unwanted noise in a system.

The disadvantages in the present flat panel speaker have led us to re-think the design principle for a flat panel speaker. Instead of applying random vibration method, if a pistonic motion as in a conventional loudspeaker be superimposed on the present flat panel speaker, the problem with low frequency and impulse response can be eliminated. The whole body motion of the panel requires hundreds of micro transducers instead of just one single transducer as present. The research looks into the feasibility of employing solenoid actuators for the novel loudspeaker design.

\section{Solenoid actuator}

The solenoid usually refers to a tube like coil only. When current passes through the solenoid, it generates a magnetic field around it. The magnetic field inside is much larger than it is outside and as a result considerable magnetic energy is stored in the interior. If a bar of permeable material is placed at one end of the solenoid, it will be drawn into the solenoid as the magnetic circuit will try to reduce the reluctance which is mostly made up by the air. The stored magnetic energy inside the solenoid is transferred 
to mechanical as a result of the plunger moving in. The actuator exploits the magnetic field in a solenoid for actuation purpose by providing an outer flux return path of permeable material and a inner flux return path through a magnetically permeable plunger which is pulled into the coil when electric current passes through the winding. The coil in the solenoid actuator creates an MMF (magneto-motive force) which drives flux through the plunger and then around the frame of the solenoid and then through the air-gap and back into the plunger. A solenoid actuator of this construction operates in one direction only and the plunger is returned only when the current is cut-off by some auxillary means such as a spring.

The force produced by the magnetic field can be explained using the following equation:

$F_{\text {mag }}=\frac{\phi^{2}}{2 \mu_{0} A_{\mathrm{g}}}=\frac{B_{\mathrm{g}}{ }^{2} A_{\mathrm{g}}}{2 \mu_{0}}$

The overall force equation governing the solenoid actuator's behaviour can be given as follows [1,2]:

$m \frac{\mathrm{d} V_{\mathrm{A}}}{\mathrm{d} t}=-c x+F_{\mathrm{mag}}+m g-F_{\text {fric }}-F_{\text {lim }}$

Here $c$ is the spring co-efficient, $m$ is the mass of the plunger, $F_{\text {fric }}$ is the friction force and $F_{\text {lim }}$ is the limiting force. The above equation has been derived assuming the actuator being in a vertical position.

\section{Experiment on a commercial solenoid actuator}

A commercial solenoid actuator was chosen to carry out primary tests on a solenoid actuator under AC condition. The initial objective of the experiment was to familiarise ourselves with the solenoid characteristics and identify the problems associated with a solenoid actuator under various operating conditions. The solenoid actuator was tested using sine waves of various frequency and amplitude levels to observe the plunger behaviour since the inputs of a loudspeaker are usually mixtures of various frequency and amplitude levels.

The plunger, top and base plate and also the surrounding frame of the solenoid actuator used in the experiment was made of mild steel and the number of coil turns was 1120. The solenoid actuator was tested with a laser vibrometer. The laser vibrometer

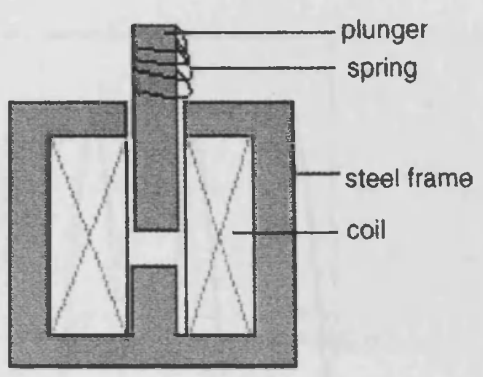

Fig. 1. Solenoid actuator.

is a hi-tech displacement measurement device with nano-metre resolution. The laser from the vibrometre was pointed to the plunger tip to measure the displacement and the output from the vibrometre was connected to a digital oscilloscope. A function generator was used to supply specific voltage and frequency to the solenoid actuator through a power amplitier. A $0.1 \Omega$ resistor was connected in series with the amplifier to measure the current and a oscilloscope was connected in parallel to the amplifier output to measure voltage supply. The block diagram of the Barkhausen noise measurement system used for control of the flux density waveform is shown in Fig. 1.

Fig. 2 shows the displacement profile of the actuator at different currents and frequencies. At low current the solenoid actuator produces linear displacement. But as we increase the current, non-linearity starts to appear in the output. The non-linearity arises from the base plate getting highly magnetized resulting from high current and then failing to de-magnetise completely before the negative cycle begins. The residual magnetism in base plate causes non-linear displacement of the plunger. As we increase the current more, displacement reduces and the plunger only shows one way movement. The reduction in displacement can be explained by the fact that at higher current the coil resistance increases due to overheating and hence receives less current and consequently produces less force which results in low displacement of the plunger. The one way movement can be explained by the fact that the electromagnetic force acts in only one direction for a solenoid actuator. The uni-directional force is higher at a higher current supply and hence compresses the spring even more. When the spring is totally compressed, it hits the base plate which is saturated at high current level, resulting in the plunger to be magnetically attached to the bottom.

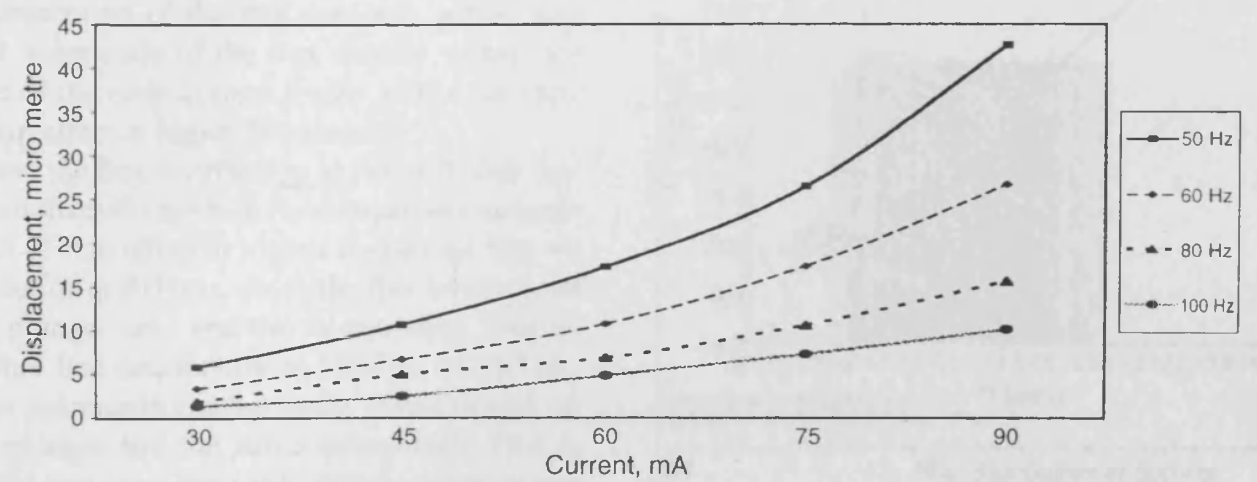

Fig. 2. Displacement profile of the solenoid actuator for 1120 turns for various frequency and current levels. 


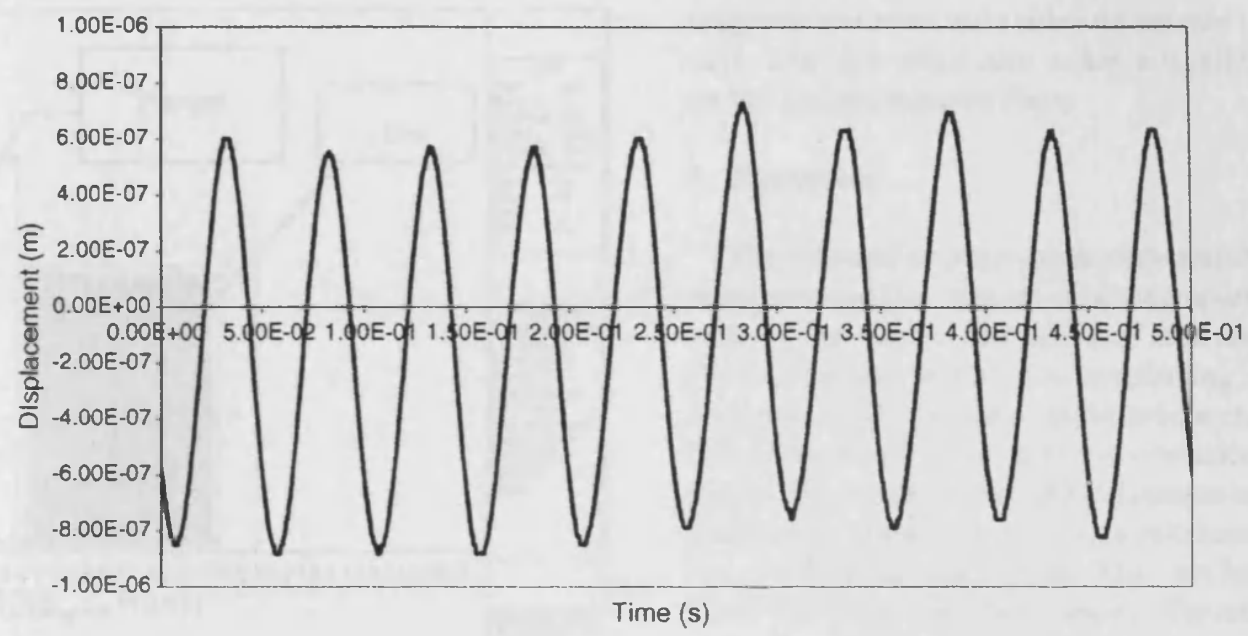

Fig. 3. Sinusoidal displacement of a solenoid ( 600 turns) for an excitation frequency of $300 \mathrm{~Hz}$.

Only during the negative input cycle, the base plate manages to demagnetise itself slightly and the spring returns slightly before the next positive cycle appear again and causes uni-directional movement of the plunger.

The next part of the experiment was to change the number of turns and observe how it affects the solenoid behaviour. For this, the number of turns was almost halved and reduced to 600 . The solenoid actuator, before and after reducing the number of turns, was tested for high frequency operation such as 200 and $300 \mathrm{~Hz}$. As expected, the 600 turns solenoid actuator could withstand the high frequencies and produce displacement (Fig. 3) whereas the 1120 turns actuator could hardly produce any displacement at these frequencies. This is obvious since fewer turns means less impedance and low impedance allows high frequency operation.

\section{FEM modelling of the solenoid actuator}

In recent days finite element modelling has widely been used for designing and understanding electromagnetic actuators. In our case, OPERA VECTOR FIELD software has been used to model the solenoid actuator and examine its behaviour. The solenoid actuator has been modelled with 1120 turns having $0.2913 \mathrm{~A} / \mathrm{mm}^{2}$ current density and the following analysis have been carried out: distribution of the flux contours within and around the solenoid. magnitude of the flux density within the solenoid, magnitude of the eddy current losses within the core and impact of the skin effect at higher frequencies.

Fig. $4 \mathrm{a}$ and $\mathrm{b}$ shows the flux distribution at two different frequencies. The simulation results are here for comparison purpose and show the impact of skin effect at higher frequency. Fig. 4a shows the flux distribution at $20 \mathrm{~Hz}$ in which the flux lines spread uniformly over the plunger area and the surrounding frames. Fig. $4 b$ shows the flux line distribution at $500 \mathrm{~Hz}$. At $500 \mathrm{~Hz}$. the flux lines are no more uniform but more concentrated on the edges over the plunger and the surrounding area. This is because the skin effect gets prominent at higher frequencies and non-uniform flux patterns start to rise.

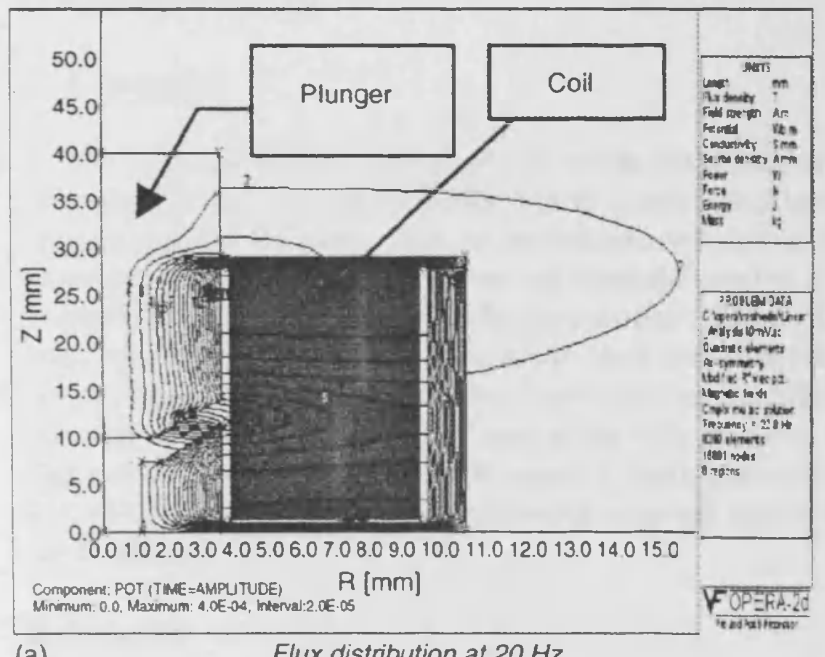

(a)

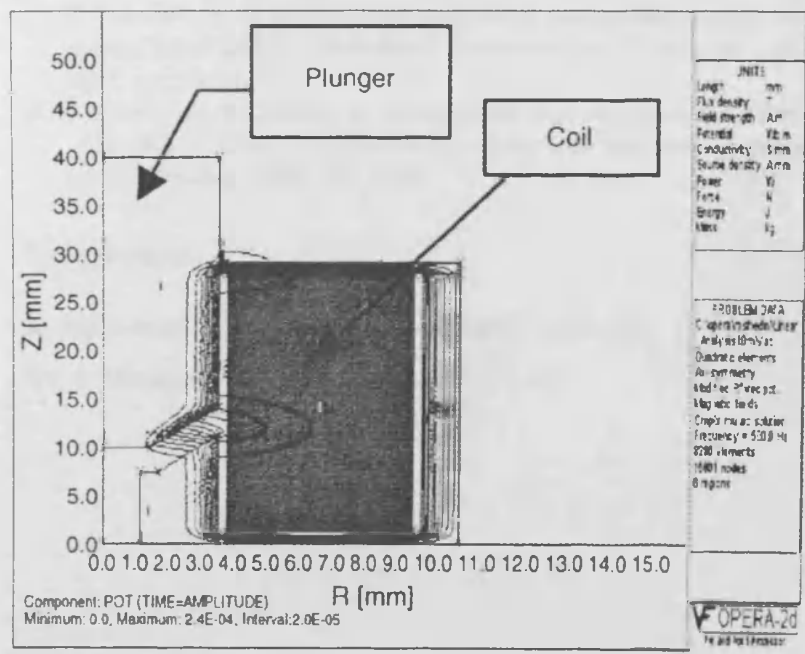

(b)

Flux distribution at $500 \mathrm{~Hz}$

Fig. 4. (a) Flux distribution at $20 \mathrm{~Hz}$, (b) flux distribution at $500 \mathrm{~Hz}$ 


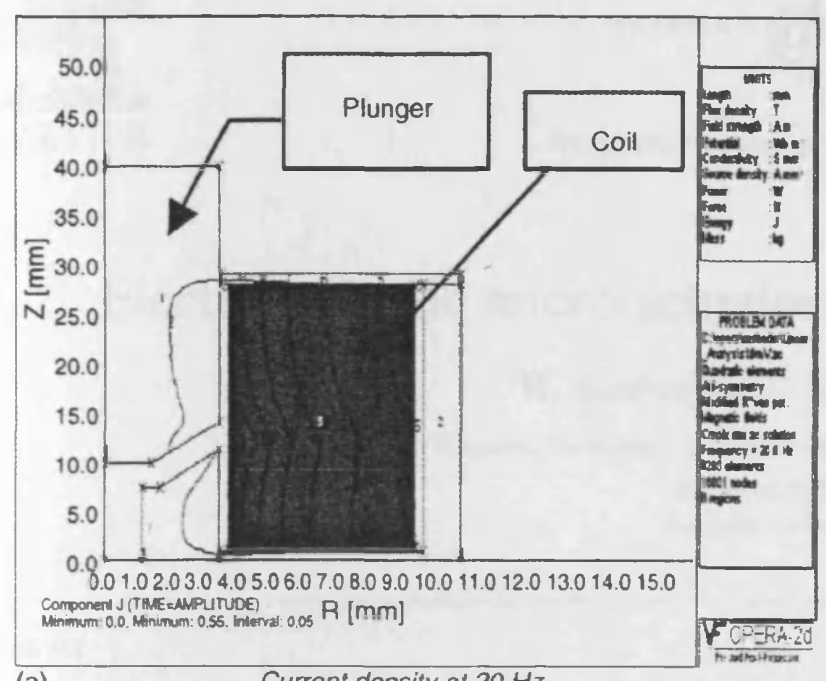

(a)

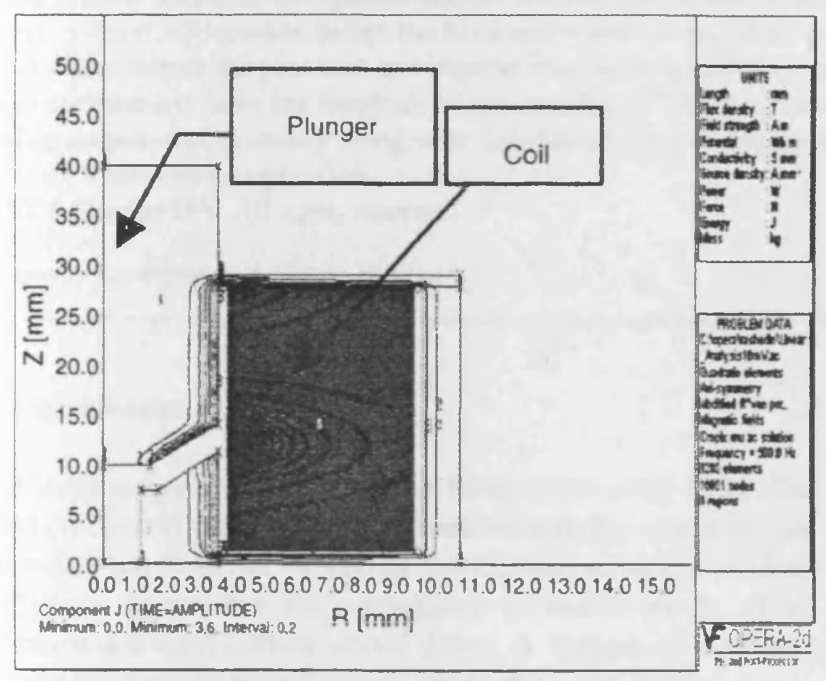

(b)

Current density at $500 \mathrm{~Hz}$

Fig. 5. (a) Current density at $20 \mathrm{~Hz}$ (b) current density at $500 \mathrm{~Hz}$.

Fig. 5a and $b$ shows the effect of eddy current as a result of the increase in frequency. At $20 \mathrm{~Hz}$ (Fig. 5a) the current density is highest in the coil and very little in the surrounding materials as expected but as the frequency is increased (Fig. 5b) current density in the magnetic materials start to increase and concentrates at the edges. The results from this FEM simulation depict the effect of eddy current and skin effect at higher frequency. The eddy current is prominent at high frequency and it reduces the overall force to drive the plunger. The eddy current loss at high frequency is a major problem in the operation of electro- magnetic actuators and a solenoid actuator is no exception in this case. The skin effect also makes a significant negative impact on the electromagnetic force.

\section{Discussion}

The solenoid actuator can be very useful in application where simplicity and low cost are of prime importance but the actuator suffers from various limitations as seen from practical tests and FEM simulation results. The surrounding iron frame introduces hysteresis in the magnetic circuit, produces eddy current loss and skin effect which are all acting as obstacles for producing linear output. Moreover, the variable reluctance of the magnetic circuit produces non-linear force-current relationship which is not ideal for a loudspeaker application. Also, not included in the simulation is the effect of the return spring. The return spring introduces instability in the system by producing unwanted oscillation. The resonant frequency of the spring, used in the commercial solenoid actuator, was $25 \mathrm{~Hz}$ and as a result the primary resonant frequency along with different harmonics of it causes oscillation in the plunger motion.

\section{Conclusion}

The simulation along with practical test on the commercial solenoid actuator reveals the limitations of a solenoid actuator operating at high frequency. Various modifications are necessary to improve the actuation behaviour if the solenoid actuator is to be used as a low frequency driver for loudspeakers. Employing materials with better magnetic properties, high saturation, high permeability, low remanence and low coercivity, laminating the material to combat eddy current loss along with applying an alternative to spring return are few possible approaches which will be considered in future for improving actuation behaviour for a solenoid.

\section{References}

[1] T. Roschke, G. Gerlach, Applied modelling and simulation, in: Proceedings of the IASTED International Conference on, 27 Jully to 1 August. 1997, pp. 241-244

[2] D. Devarajan, S. Stanton, B. Knorr, Behavioral modelling and simulation 2003 (BMAS 2003), in: Proceedings of the 2003 International Workshop on 7-8 October, 2003 , pp. $76-81$.

\section{Biographies}

R. Rashedin is a Research Student at Cardiff University.

Dr. T. Meydan is a Reader at Cardiff University. 


\title{
Electromagnetic micro-actuator array for loudspeaker application
}

\author{
R. Rashedin, T. Meydan*, F. Borza \\ Wolfson Centre for Magnetics Technology, Cardiff University, P.O. Box 925, Newport Road, Cardiff CF24 OYF, UK \\ Received 23 September 2005 \\ Available online 24 January 2006
}

\begin{abstract}
The present flat panel loudspeaker exploits the resonant modes whereas conventional cone type loudspeaker uses pistonic motion to reproduce sound. A novel loudspeaker design has been undertaken to introduce pistonic motion in flat panel loudspeakers. The whole body vibration of the panel will eliminate the poor bass and impulse response associated with the present flat panel speakers. Instead of one or two exciter, the proposed panel speakers will have few hundreds micro-transducers vibrating coherently to produce sound. A matrix of miniature electromagnetic actuators having magnet-coil assembly along with simulation using commercial simulation packages explores the possibilities of using micro-actuators array for loudspeaker application.
\end{abstract}

(ㄷ) 2005 Elsevier B.V. All rights reserved.

Keyuords: Loudspeaker; Actuator; Voice coil

\section{Introduction}

Electromagnetic actuators have been considered to be the most efficient in various actuation mechanisms but miniaturized operation has been an exception. Nevertheless, recent studies $[1,2]$ have shown that electromagnetic actuators can be more effective and useful when scaled down. A magnet of a certain polarization. when scaled down, exerts the same field at a distance reduced by the same amount as the magnet itself. This phenomenon has important consequences since the conductor that interacts with the magnet experiences the same force as before because of the constant field.

The current carrying conductors, on the other hand, are more efficient in terms of heat dissipation when scaled down. The joule losses that heat up the conductors are proportional to the volume but the heat dissipation is proportional to the surface area, so when a conductor is scaled down, the heat generation to heat dissipation ratio decreases. The decrease in heating results in the increase of current density of conductors within the admissible thermal limits.

A current-magnet combination for a miniaturized actuator appears to be the most capable for audio application after theoretical assumptions and practical experiments on a solenoid

\footnotetext{
- Corresponding author. Fax: +442920879538.

E-mail address: meydan@ cardiff.ac.uk (T. Meydan).
}

actuator (a current-magnetic material combination) and a moving voice coil (a current-magnet actuator) proves the latter to be more effective in audio frequency operation.

For proof of principle, a miniature electromagnetic actuator has been developed that can operate over the audible frequency range. The actuator is a moving magnet placed on a high permeability amorphous material which acts as the panel for the speaker. The other part of the actuator is a $7 \mathrm{~mm}$ diameter coil attached to the bottom layer made of amorphous material. The permanent magnet is a Neodymium Boron Iron magnet, $6 \mathrm{~mm}$ in diameter and is enclosed by a $0.12 \mathrm{~mm}$ diameter copper wire coil. The actuator was excited with a sine wave of frequency varying from $20 \mathrm{~Hz}$ to $20 \mathrm{kHz}$ and the resulting displacement of the panel was measured using a laser vibrometer. Opera, a Finite Element Modelling software [Vector Fields], was used to simulate and analyze the behaviours of the device. The simulation software allowed optimization of the geometry and of the materials to be used. A $2 \times 2$ array of micro-actuators was then developed for experimentation. Further improvements then followed in a $9 \times 2$ array loudspeaker.

\section{Experimentation}

The new actuator, described in Section 1, was tested for the audio frequency using a sine wave and the displacements of 


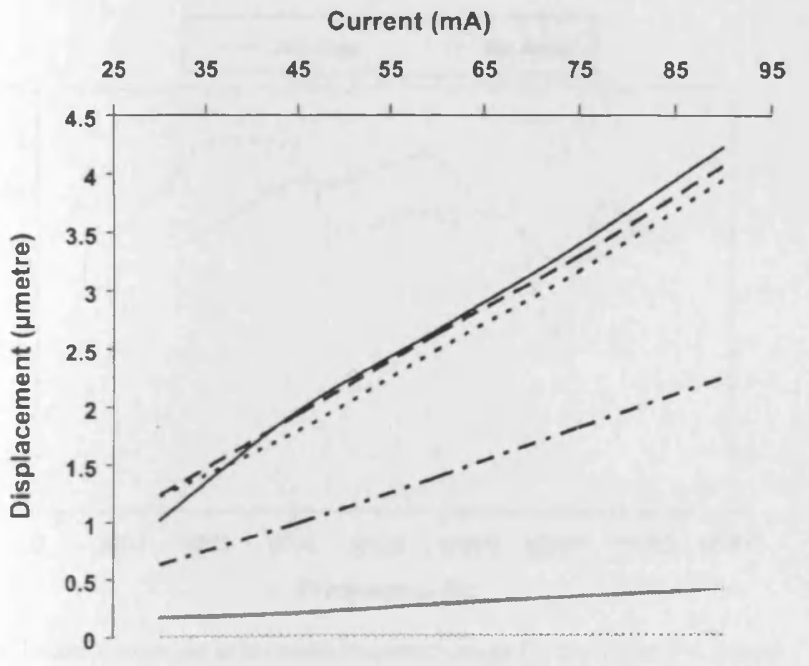

$-20 \mathrm{~Hz} \cdots 50 \mathrm{~Hz}--100 \mathrm{~Hz}-\cdot 500 \mathrm{~Hz}-1 \mathrm{kHz}-3 \mathrm{kHz}$

Fig. 1. Displacements at frequencies varying from $20 \mathrm{~Hz}$ to $3 \mathrm{kHz}$.

the front panel have been measured using an OFV-303 laser vibrometer.

The laser was pointed to the front panel to measure the displacement and the output from the vibrometer was connected to a digital oscilloscope. A function generator was used to supply specific voltage and frequency to the actuator through a power amplifier. A $0.1 \Omega$ resistor was connected in series with the amplifier to measure the current and an oscilloscope was connected in parallel to the amplifier output to measure voltage supply. Fig. 1 shows the displacements at various frequencies at different levels of currents. The frequencies have been varied from $20 \mathrm{~Hz}$ to $3 \mathrm{kHz}$ whereas for each frequency the current level was varied from 30 to $90 \mathrm{~mA}$. The actuator shows linear response at each of the frequencies for various currents. This linear displacement versus current response is ideal for a loudspeaker application.

Responses at high frequencies show similar linear characteristics for the actuator at different current levels. Fig. 2 shows the displacement profile with the frequency varying from $20 \mathrm{~Hz}$ to $20 \mathrm{kHz}$. The displacement gradually decreases as the frequency increases.

The results from the vibration measurement indicate that the actuator has a linear response over the whole audible frequency range. The peak displacement of the magnet-coil assembly reaches $4.25 \mu \mathrm{m}$ at $90 \mathrm{~mA}$. The sound radiating from the vibrating panel is clearly audible from $300 \mathrm{~Hz}$ to $15 \mathrm{kHz}$. There is a slight increase in impedance with the frequency as a result of the increase in the coil resistance due to skin effect at high frequency.

\section{Simulation}

The flux pattern of the designed electromagnetic actuator was observed using finite element modelling and simulation. Fig. 3 shows FEM generated flux line distribution for the actuator.

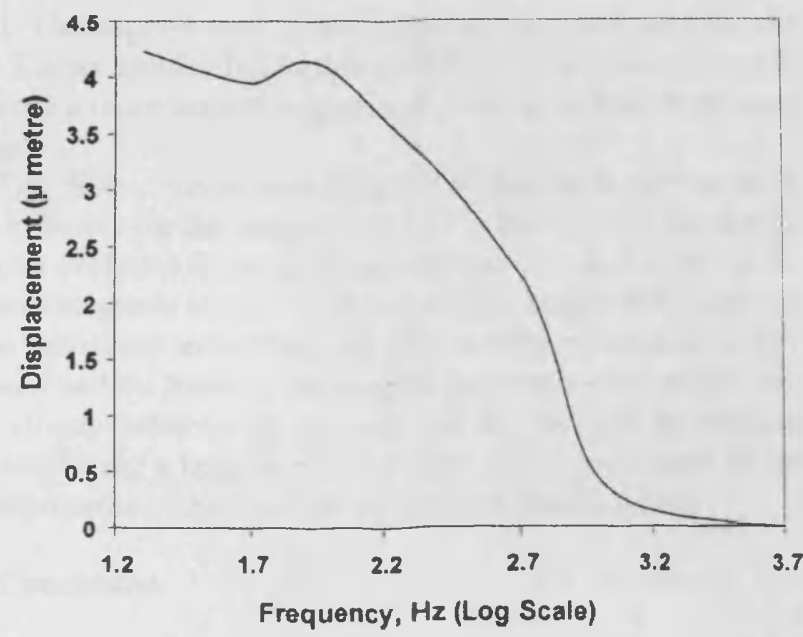

Fig. 2. Displacement profile for the actuator at the audible frequency range

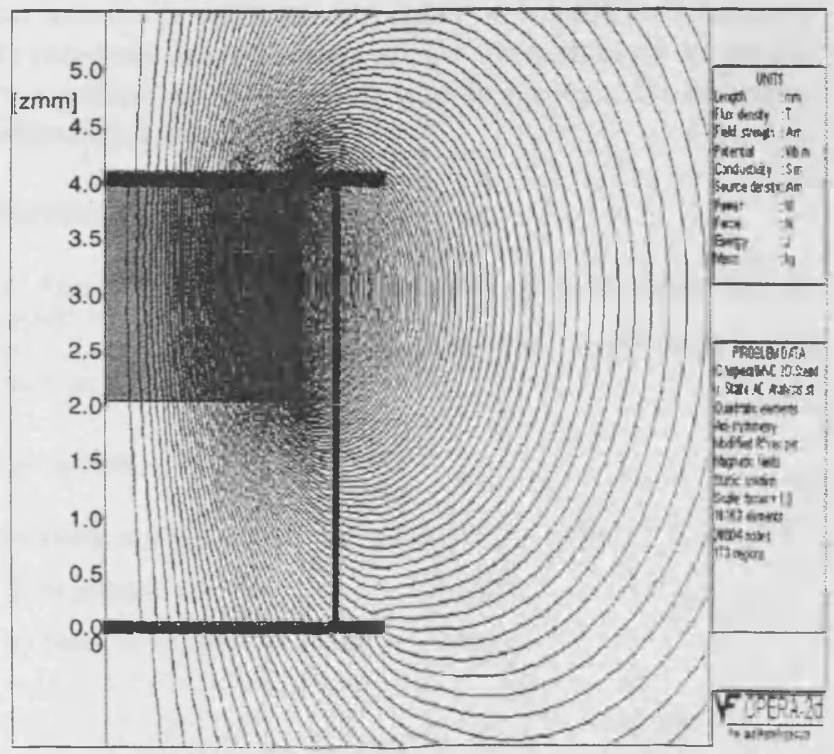

Fig. 3. Flux lines distribution using FEM

The simulation shows that the flux is concentrated in the upper right corner of the actuator. Most of the flux passes through the upper panel associated with the magnet and hence creates the maximum flux density on its return path at the right hand corner. The bottom panel is not very effective in terms of flux concentration; firstly because there is a large air gap bet ween the magnet and the bottom panel and secondly because the panel is only $0.02 \mathrm{~mm}$ thick and hence has a considerable electrical resistance associated with it.

\section{Measurement of pressure response}

The pressure response from both the $2 \times 2$ array and the $9 \times 2$ array have been measured using a high bandwidth microphone. Both the speakers were fed with a sine wave of frequency varying from $20 \mathrm{~Hz}$ to $20 \mathrm{kHz}$. The pressure response from the front panel was recorded by a microphone connected to an A/D card 


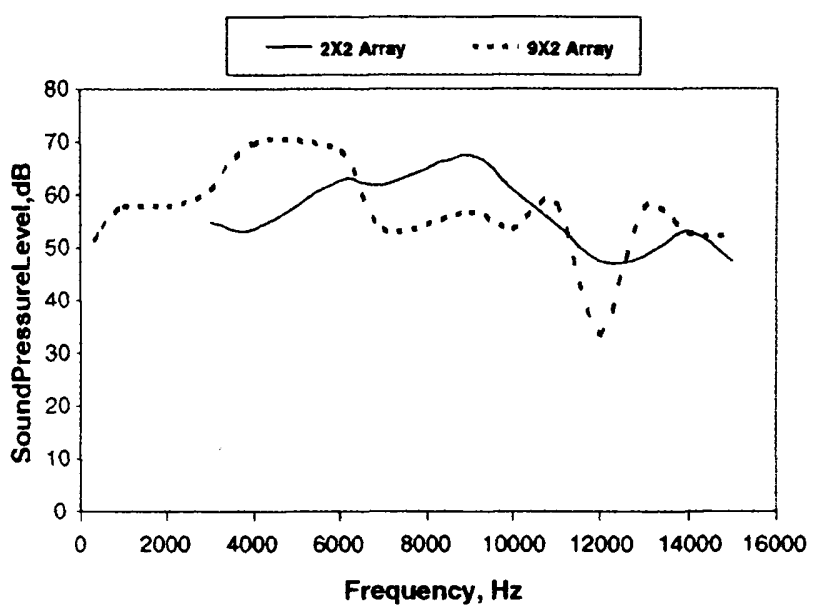

Fig. 4. Pressure response at the audio frequency range for $2 \times 2$ and $9 \times 2$ array speaker.

to the computer. The data was then acquired and processed using Labview Software. The pressure response in both cases reaches $70 \mathrm{~dB}$ peak but the frequency at which the maximum occurs shifts to the lower frequency region in the case of $9 \times 2$ array speaker. Also the $9 \times 2$ array speaker has better response in the bass region compared to the $2 \times 2$ array speaker at the same distance (Fig. 4).

\section{Discussion}

The initial results for the pressure response show the improvement in bass response for longer array speaker. The $9 \times 2$ array speaker was impedance matched at $8 \Omega$ for standard amplifiers whereas the $2 \times 2$ array was of $0.7 \Omega$ because of its parallel connection. Also because the panel of the $9 \times 2$ array speaker has a larger surface area and has more actuators attached to it than the $2 \times 2$ array speaker, it was able to drive more air mass in front of it. The improvement in bass response was expected from the $9 \times 2$ array speaker but further modification will be required to achieve a more smooth response all over the audible frequency range.

The finite element modelling shows that the bottom layer is not effective for the magnetic circuit in the actuator and hence can be excluded from the future design. The exclusion of the bottom magnetic material will result in the height of the actuator to be halved and reduced air-gap. This will ensure increase in flux density and the force on the magnet-panel assembly. Moreover, the air-gap between the magnet and the coil can be reduced by employing a large number of turns which will result in the improvement of the loudness of the loudspeaker system.

\section{Conclusion}

The novel transducer proposed in this paper has the desired characteristics for loudspeaker application. It was shown that the multi-actuator array as a loudspeaker can significantly improve bass response. Future design will accommodate all the necessary modifications to ensure a smooth response for the novel loudspeaker system.

\section{References}

[1] K. Kim, H. Yoon, O. Jeong, S. Yang, Sens. Actuators A: Phys. 117 (1) (2005) 241-244.

[2] O. Cugat. J. Delamare, G. Reyne, IEEE Trans. Magn. 39 (6) (2003) $3607-3612$.

\section{Biographies}

R. Rashedin is a Research Student at Cardiff University.

Dr. T. Meydan is a Reader at Cardiff University.

Dr. F. Borza is a Lecturer at Cardiff University. 


\title{
A Novel Miniature Matrix Array Transducer System for Loudspeakers
}

\author{
R. Rashedin, T. Meydan, and F. Borza \\ Wolfson Centre for Magnetics, School of Engineering, Cardiff University, Cardiff CF24 3AA, U.K.
}

\begin{abstract}
A breakthrough in novel loudspeaker design has been achieved using miniature actuators in a matrix configuration. Present flatpanel loudspeakers exploit resonant modes, whereas conventional cone-type loudspeakers use pistonic motion to reproduce sound. A new actuator has been designed so that it introduces pistonic motion in flat-panel loudspeakers. Instead of one or two exciters, the proposed panel speaker has numerous miniature transducers vibrating coherently to produce sound. In this device, the whole body vibration of the panel reduces the poor bass and impulse response associated with present flat-panel speakers. Frequency responses show that this novel transducer is suitable for loudspeaker application because of its linear and consistent output over the whole audible frequency range and for various excitation currents. Measurements on three different device configurations prove a moving magnet transducer to be the most suitable for loudspeaker application. Finite element modeling has been used to examine single transducer operation and the magnetic interaction between neighboring transducers in a matrix array format. Experimental results show the correct positioning of the transducers in a matrix configuration, as observed by the simulation, reduces the effects of interferences on the magnetic transd ucers. Also, results show that larger matrix array transducer speakers exhibit better low-frequency responses.
\end{abstract}

Index Terms-Arrays, electromagnetic actuators, frequency response, magnetic devices, magnetic materials, modeling.

\section{INTRODUCTION}

C ONVENTIONAL loudspeakers, by employing wholebody vibration of the diaphragm, can reproduce good quality sound at the low end of the audio spectrum, i.e., below $1 \mathrm{kHz}$. Flat-panel speakers, on the other hand, are better at high-frequency operation as the reproduced sound at high frequency from a flat-panel speaker is not omnidirectional as in the case of a conventional loudspeaker [1].

Although flat-panel speakers are compact, small, and have a better high-frequency response, the poor reproduction of bass sound limits its performance severely. Also, the flat-panel speakers have a poor impulse response. The reason for such poor bass and impulse response is that, unlike the whole-body movement of a conventional loudspeaker diaphragm, different parts of the panel in a flat-panel loudspeaker vibrate independently. Therefore, the new design aims to introduce whole-body motion in flat-panel speakers [2]. Instead of a single exciter, the proposed miniature matrix array transducer system for the loudspeaker will employ numerous miniature transducers to vibrate coherently and produce sound effectively at the low and high range of the audio spectrum. The multiactuator approach combines the advantages of conventional whole-body motion with that of modern flat-panel speakers. This new loudspeaker design will also provide improvement in impulse response.

Electromagnetic transducers, due to their high power output, have been the most effective in loudspeaker application, and recent studies [3] have shown that electromagnetic actuators can be even more effective and useful when scaled down. Also a current-magnet combination for a miniaturized electromagnetic actuator appears to be the most capable for audio applications after a theoretical analysis and practical experiments were undertaken on a solenoid actuator (a current-magnetic material

Digital Object Identifier 10.1109/TMAG.2006.879073 combination) and a moving voice coil (a current-magnet actuator) [4], where the latter seems to be more effective in audio frequency operation. Therefore, miniature novel electromagnetic transducers having a current-magnet combination have been used to construct a matrix of transducers that can work as a miniature matrix-array loudspeaker. Three different device configurations of this novel loudspeaker have been investigated in this paper for performance optimization.

\section{Design AND Development}

\section{A. Novel Transducer}

An innovative miniature actuator for the proposed loudspeaker has been designed, modeled, and built for analysis. This novel transducer speaker consists of a permanent magnet placed on a high-permeability amorphous magnetic ribbon that acts as the panel for the speaker. In addition, a 30-turn copper wire coil attached to another amorphous magnetic ribbon supplies the drive field and flux closure, respectively [2]. The permanent magnet is a Neodymium Boron Iron magnet, $6 \mathrm{~mm}$ in diameter and oriented normal to the attached surface, is enclosed by the 0.12 -mm-diameter copper wire coil. The overall size of each pixel is $8.5 \times 8.5 \times 4.5 \mathrm{~mm}$. The proposed new loudspeaker was developed using these novel actuators in a matrix configuration. A $2 \times 2$ and then a $9 \times 2$ array of miniature actuators were developed for experimentation. Measurements on a single transducer have been carried out on three different device configurations in order to optimize the device. The three different configurations were 1) moving magnet, 2) moving coil, and 3) moving magnet with a plastic "bottom layer" instead of the amorphous ribbon.

\section{B. Simulation}

The FEM simulation tool "MAGNET" was used to characterize the transducer's magnetic performance. For a single-cell transducer, under static condition, the simulation showed the flux distribution, flux density at various parts, and the force distribution (see Fig. 1). 


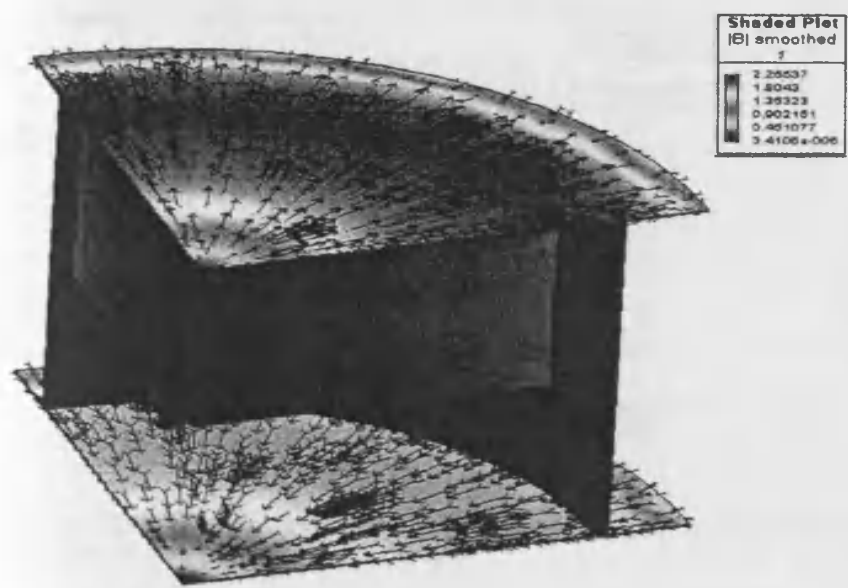

Fig. 1. Cross-sectional model of the transducer showing flux distribution.

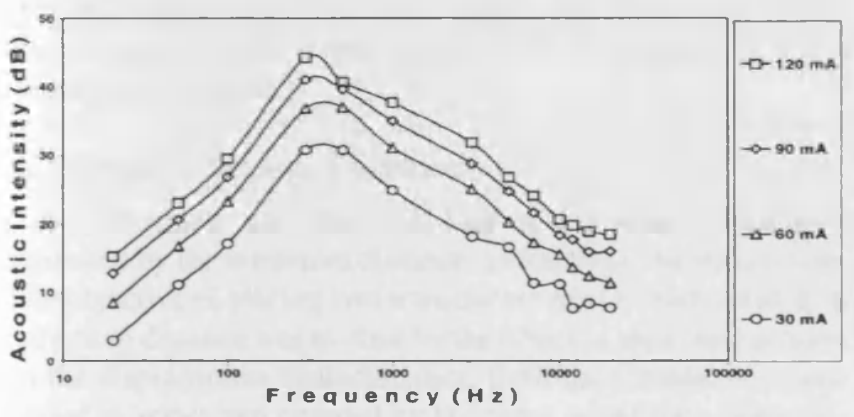

Fig. 2. Acoustic intensity levels for the moving magnet combination.

Two magnets when placed alongside repel each other, and this repelling force determines the minimum distance between them. Finite element modeling has been used to determine the force profile and the minimum distance between the transducers in the matrix array loudspeaker. The closest proximity achievable between any two transducers within the matrix array was found to be $0.5 \mathrm{~mm}$.

\section{RESULTS}

\section{A. Experimental Set-Up}

The new speaker was tested for audio frequency response using a sine wave signal output. The displacements of the front vibrating panel of a single transducer were measured using an OFV-303 laser Doppler vibrometer. The laser was incident on the vibrating panel to measure the displacement, and the output from the vibrometer was processed by a lock-in amplifier. The frequencies have been varied from $20 \mathrm{~Hz}$ to $20 \mathrm{kHz}$ with excitation current levels from 30 to $120 \mathrm{~mA}$.

\section{B. Acoustic Pressure Responses}

The results from displacement measurement indicate that the actuator has a linear displacement versus current relationship over the whole audible frequency range [2]. This linearity is essential if the device is to operate as a broad bandwidth loudspeaker. Based on the acoustic intensity (mean acoustic power transmitted through a unit area) measurements (see Figs. 2-4), the sound levels at high frequencies are higher for the moving

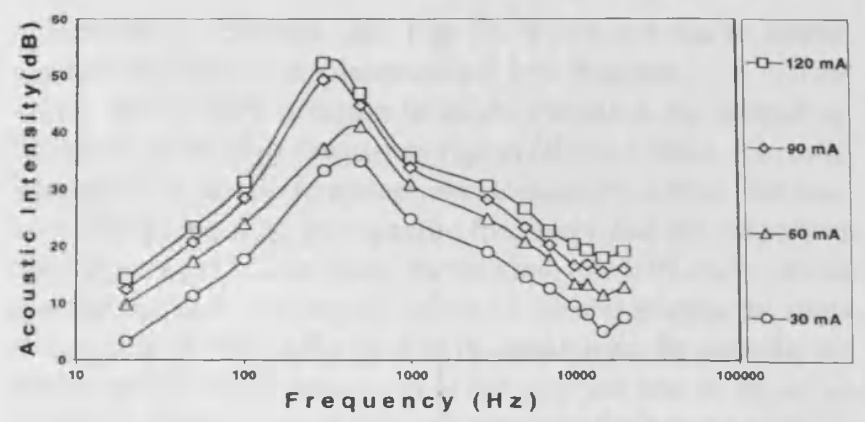

Fig. 3. Acoustic intensity levels for the moving magnet (plastic "bottom layer") combination.

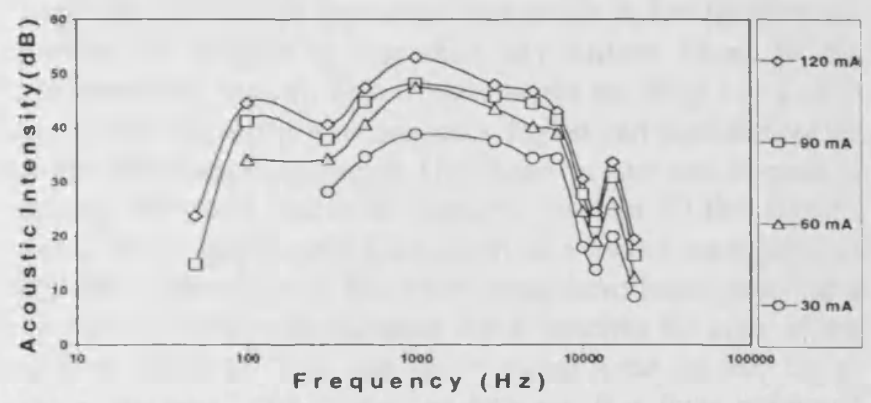

Fig. 4. Acoustic intensity levels for the moving coil combination.

coil arrangement than for the other two device configurations. The moving coil arrangement, however, has a low frequency cut-off at $300 \mathrm{~Hz}$ for lower excitation currents and resonance at $15 \mathrm{kHz}$ (see Fig. 4). The other two combinations operate better at as low as $20 \mathrm{~Hz}$. Based on the low-frequency operation, displacement amplitude, and mechanical stability, the moving magnet configuration is the most suitable for loudspeaker application.

\section{Impedance Measurement}

Impedance measurements of a single transducer show an increase in impedance as the frequency changes from $20 \mathrm{~Hz}$ to $20 \mathrm{kHz}$. The impedance increases by only $7 \%$ over the whole audible frequency range and hence has no significant effect on the performance of the loudspeaker. The inductance remains constant, and since the value of inductance is very low compared to the resistance of the coil, the reactance that increases with the frequency does not have an effect on the overall impedance.

\section{Improvement of Bass Response}

Pressure responses from a $2 \times 2$ array and a $9 \times 2$ array matrix speaker have been measured using a high bandwidth microphone, and the results were published elsewhere [2]. Both the speakers were driven with the same excitation current. For the $2 \times 2$ array speaker, the lowest frequency observed was $3 \mathrm{kHz}$, and below this frequency, the sound pressure was too low to be measured by a high bandwidth microphone. For the $9 \times 2$ array speaker, however, the lowest frequency at which sound pressure could be measured was around $50 \mathrm{~Hz}$. The $9 \times 2$ array speaker has better acoustic pressure response in the bass region compared to the $2 \times 2$ array speaker at the same distance. The results from the pressure response measurement show the improvement 


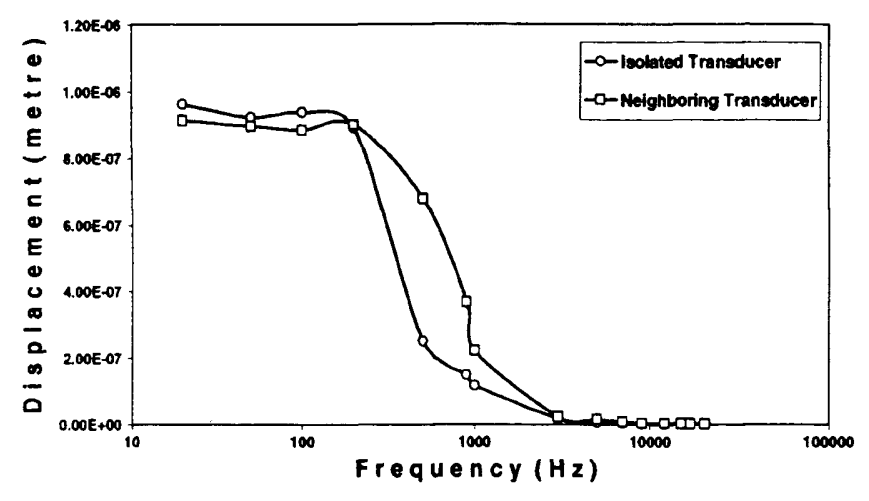

Fig. 5. Displacement characteristics for an isolated and a neighboring transducer for the audible frequency range.

in bass response for the $9 \times 2$ array speaker. A larger surface area and higher force obtained by employing more active transducers resulted in the improvement of the bass response for the longer array speaker.

\section{E. Interaction Between Transducers}

A loudspeaker was constructed using two novel transducers separated by the minimum distance, as found by the simulation. The objective of placing two transducers next to each other at a minimum distance was to observe the effect of their interactions on the displacement characteristics. Both the transducers, connected in series and coupled by the same membrane, were excited by an excitation current of $120 \mathrm{~mA}$. The displacements of a single transducer from this setting have been measured, and the results (see Fig. 5) show that the displacement profile matches closely to that of an isolated single transducer. The influence of the mechanical coupling is not significant since similar results have been obtained by placing alongside two separate transducers that are not coupled by a continuous membrane. Therefore, electromagnetic transducers separated by a minimum distance have no effect on their individual displacement characteristics. as is evident from the experimental results.

\section{ANALYSIS}

The results from the three different device configurations show that a moving magnet combination is the best arrangement for loudspeaker application because of its mechanical stability and higher output displacements. The lower amorphous panel and the magnet attract each other and hence give mechanical stability to the transducer. For a plastic bottom layer, mechanical instability rises because of the lack of a holding force between the vibrating diaphragm and the lower panel. The moving coil transducer, although having higher displacements at high frequencies, possesses relatively low mass, and the small size of the coil results in an acoustic impedance mismatch at low frequency and prevents it from producing sounds at the low end of the audio spectrum.
Frequency response (see Fig. 5) of the transducer shows gradual decrease in displacements at low frequency, i.e., below $1 \mathrm{kHz}$, and a sharp decrease in displacements as the frequency increases in the high-frequency region (above $1 \mathrm{kHz}$ ). Acoustic intensity at a certain frequency is calculated by a term that consists of a product of that specific frequency and the respective displacement [1]. Therefore, the acoustic intensity curve shows gradual increase in intensity below $1 \mathrm{kHz}$ and gradual decrease in intensity above $1 \mathrm{kHz}$. Due to the small size, the acoustic intensity of the single transducer at the very low end of the audio spectrum (below $300 \mathrm{~Hz}$ ) is not adequate for human hearing. The low-frequency limitation for a single transducer is evident from the actuator intensity levels (see Figs. 2-4) since, for a single transducer, the low sound intensities in low frequencies prevent the speaker to reproduce any audible sound in the low-frequency region. This is because the ear drum has a very low sensitivity at the low-frequency region and high sensitivity at the high-frequency region [1]. However, the low-frequency hearing threshold has been improved (around $50 \mathrm{~Hz}$ ) significantly by using the same transducers in a matrix configuration [2]. The transducers in the matrix array have been placed at a distance in which the repelling force matches the sum of the surface attraction force and the frictional force. At this "minimum distance," the interaction between flux lines produced by the neighboring transducers is not significant, as confirmed by the simulation result. Since minimization of independent movements in the diaphragm of a flat-panel speaker can only be achieved by placing numerous miniature transducers in the closest proximity possible, this minimum distance between the transducers will ensure maximum whole-body vibration.

\section{CONCLUSION}

The matrix configuration of miniature transducers improves the low-frequency response. The efficiency and sensitivity of miniature loudspeakers has been improved by means of loudspeakers with a large diaphragm area, high flux density, a high number of turns, a small voice coil resistance, and small mass. The large diaphragm in a larger array speaker has extended the low-frequency operation down to $50 \mathrm{~Hz}$. In a matrix array loudspeaker, electromagnetic transducers can operate without any mutual disturbance when separated by a minimum distance.

\section{REFERENCES}

[1] J. M. Eargle, Loudspeaker Handbook, 2nd ed. Norwell, MA: Kluwer, 2003.

[2] R. Rashedin, T. Meydan, and F. Borza, "Electromagnetic micro-actuator array for loudspeaker application," Sens. Actuat. A: Phys., to be published.

[3] O. Cugat, J. Delamare, and G. Reyne, "Magnetic micro-actuators and systems (MAGMAS)," IEEE Trans. Magn., vol. 39, no. 6, pp. 3607-3612, Nov. 2003

[4] R. Rashedin, T. Meydan, and F. Borza, "Solenoid actuator for loudspeaker application," Sens. Actuat. A: Phys., to be published.

Manuscript received March 12, 2006 (e-mail: RashedinR1@cardiff.ac.uk). 


\section{ANALYSIS OF A MOVING MAGNET MINIATURE TRANSDUCER ARRAY LOUDSPEAKER SYSTEM}

\author{
Razib Rashedin \\ Wolfson Centre for Magnetics \\ School of Engineering \\ Cardiff University \\ Cardiff, CF24 3AA \\ email: RashedinR1@cardiff.ac.uk
}

\author{
Dr. Turgut Meydan \\ Wolfson Centre for Magnetics \\ School of Engineering \\ Cardiff University \\ Cardiff, CF24 3AA \\ email: Meydan@cardiff.ac.uk
}

\begin{abstract}
A novel electromagnetic transducer speaker, that aims to employ whole body vibration of the panel to reduce the poor bass and impulse response associated with present flat panel speaker, have been tested and analyzed for the audible frequency range. Frequency response of a moving magnet configuration of this device shows consistent and linear output for various excitation current levels over the whole audible frequency range. Phase and magnitude measurement shows existence of resonance at the very low end i.e. below $1 \mathrm{kHz}$ of the audio spectrum.
\end{abstract}

Finite element modelling has been used to examine single transducer operation and the magnetic interaction between neighboring transducers in a matrix array format. Experimental results show the correct positioning of the transducers in a matrix configuration, as observed by the simulation, reduces the effects of interferences on the magnetic transducers. Also, results show that larger matrix array transducer speaker exhibit better low frequency response.

\section{INTRODUCTION}

Present flat panel loudspeakers exploit resonant modes whereas conventional cone type loudspeakers use pistonic motion to reproduce sound [1]. By employing pistonic or whole body vibration of the diaphragm, conventional speakers can produce low frequency sound (below $1 \mathrm{kHz}$ ) effectively. But since flat panel speaker uses resonant modes of operation, it suffers from poor bass response despite having better high frequency response than the conventional ones. Therefore, a separate sub-woofer is usually employed to boost the bass sound in a flat panel speaker. Moreover, because of the resonant mode, flat panel speakers have poor impulse response. The new miniature transducers speaker array is designed so that it introduces pistonic motion in flat panel loudspeakers. The multi-actuator approach combines the advantages of conventional whole body motion with that of modern flat panel speakers.

A novel electromagnetic transducer has been developed for the proposed matrix array loudspeaker [2]. Three different device configurations of this new device namely the moving magnet, the moving coil and the moving magnet with a plastic bottom layer instead of an amorphous one have been tested for acoustic intensity responses and mechanical stability and the results show that a moving magnet seems to be the most effective transduction mechanism for the loudspeaker application [3]. The frequency response, measurement of resonance and the analysis of magnetic interaction between neighboring transducers in a matrix array loudspeaker further proves the suitability of employing this moving magnet transducer in a matrix array speaker configuration.

\section{DESIGN AND DEVELOPMENT}

The novel electromagnetic transducer speaker has been designed and developed using the principle of two interacting magnetic fields. The vibrating panel of the transducer speaker is a high permeable amorphous ribbon attached to a permanent magnet $(\mathrm{Nd}-\mathrm{Fe}-\mathrm{B})$ that provides a static magnetic field. The other alternating magnetic field is produced by the coil which is also attached to another amorphous material [2]. The operation characteristics of this novel moving magnet transducer have been observed and analyzed both by simulation and practical experiments. Matrix array speakers developed from these transducers have also been tested for understanding the acoustic behaviour, magnetic interactions and the advantages of a multi actuator approach. Finite element modelling and experimental results explore various device configurations, frequency responses, resonance and the nature and effects of magnetic interactions between transducers.

\section{A. Operation Principle}

Simulation tool 'MAGNET' was used to characterize the transducer's magnetic performance. For a single cell transducer, under static condition, the simulation showed the flux distribution, flux density at various parts and the force distribution.

For the single cell transducer, the upper and the lower magnetic material together with the permanent magnet $(\mathrm{Nd}$ Fe-B) form a magnetic circuit. The excitation alternating current to the coil generates an alternating magnetic field that interacts with the static magnetic field of the permanent magnet. The interaction between the two fields causes an 'attract' and 'repel' action that translates to mechanical vibration of the panel. The instantaneous force that governs the displacement of the panel is given by the following equation. 


$$
F=B i l \sin \theta
$$

Here $B$ is the magnetic flux density in the air gap due to the permanent magnet, $i$ is the instantaneous excitation current, $l$ is the length of the coil and $\theta$ is the angle of interaction between the two magnetic fields.

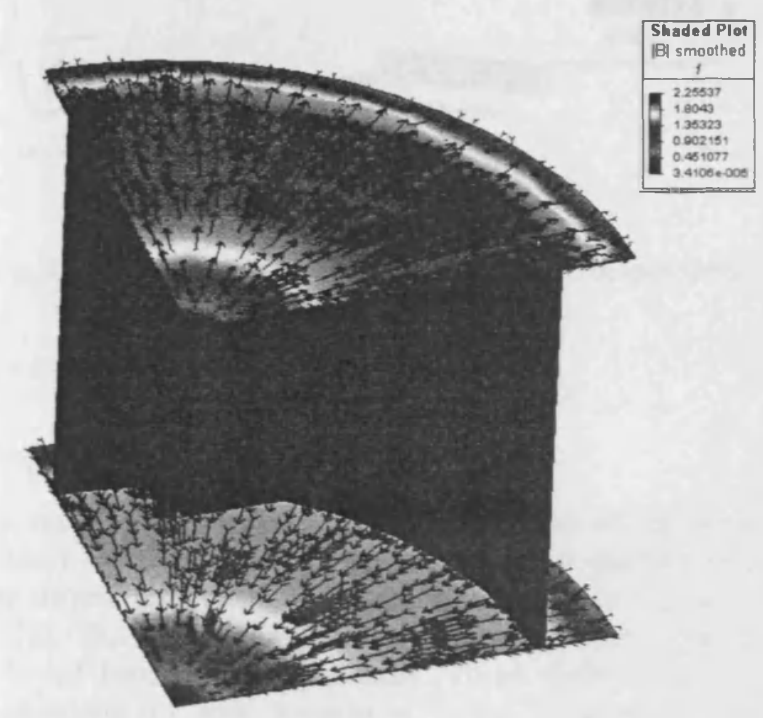

Fig. 1. A quarter model of the transducer showing flux density and flux distribution

The flux distribution of the transducer (Fig. 1) shows the flux circulating between the upper and the lower panel. The flux from the magnet flows through the upper panel and then returns via the lower panel of the transducer. There is a considerable amount of flux leakage due to the air gap present between the two panels. A minimum air-gap is required to separate the magnet from the lower panel. The simulation shows that the concentration of the flux on the upper panel increases from the centre towards the periphery of the magnet. This increase of flux density is due to the coil flux adding up to the flux from the permanent magnet.

The positioning of the permanent magnet within the magnetic circuit determines the magnitude of force that vibrates the diaphragm. Therefore the magnet was placed at different positions within the air-gap to observe the force profile. The simulation result (Fig.2) shows the ideal placement of the magnet for maximum force. It should be noted that at a certain position i.e. at the middle, the interacting fields cancel each other and there is a null force as seen in the following force profile.

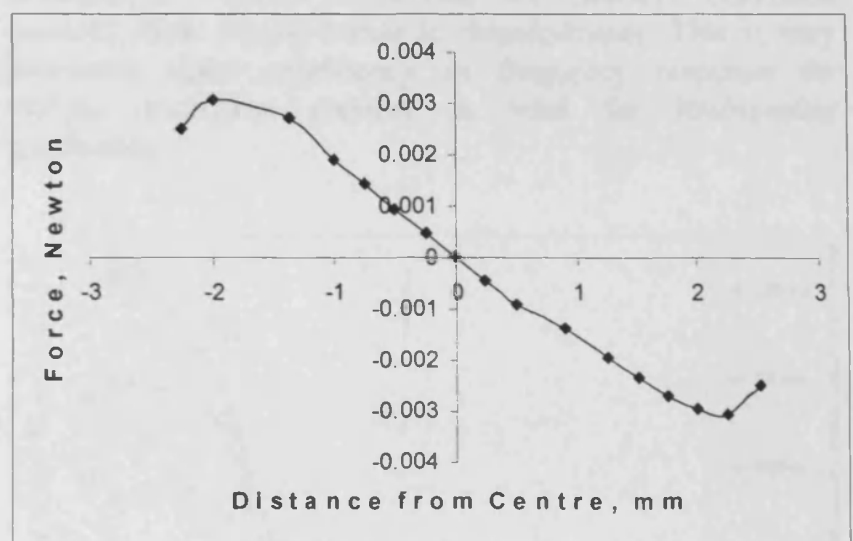

Fig. 2. Force profile in the air gap for different positioning of the permanent magnet

\section{B. Matrix Array}

Two magnets when placed alongside repel each other and this repelling force determines the minimum distance between them. Finite element modelling has been used to determine the force profile and the minimum distance between the transducers in the matrix array loudspeaker (Fig. 3). The repelling force between the permanent magnets limits the proximity between electromagnetic transducers. The maximum proximity achievable between any two transducers within the matrix array was found to be $0.5 \mathrm{~mm}$. This minimum distance between the transducers will ensure negligible repelling force and the maximum whole body vibration.

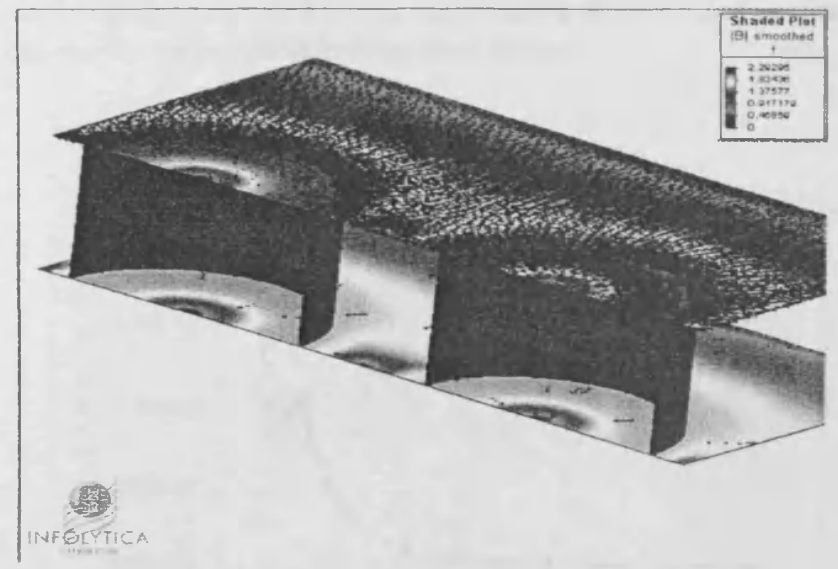

Fig. 3. Interaction between transducers in the matrix array

\section{Experimental Set-up}

The new transducer was tested in the audio frequency range by applying a sine wave current to the drive coil, with the resultant displacement of the front face measured using 
an OFV-303 laser vibrometer coupled to a digital lock-in amplifier. The frequency was then varied from $20 \mathrm{~Hz}$ to 20 $\mathrm{kHz}$ with excitation currents levels from $30 \mathrm{~mA}$ to $120 \mathrm{~mA}$.

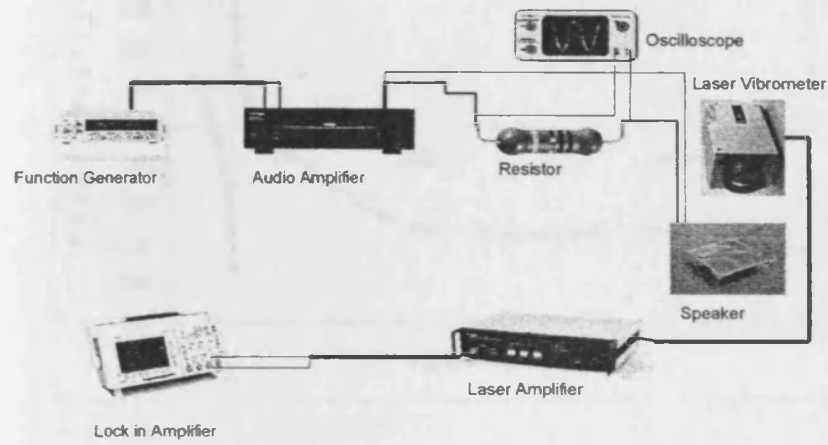

Fig. 4. Experimental set-up for the displacement measurement using laser vibrometer

\section{Frequency Responses}

The results from displacement measurement of the novel transducer indicate that the actuator has a linear displacement versus current relationship over the whole audible frequency range [2]. This linearity is essential if the device is to operate as a broad bandwidth loudspeaker. Three different device configurations of this transducer have been tested for acoustic intensity levels and the results indicate that a moving magnet combination is best suitable for loudspeaker application [3].

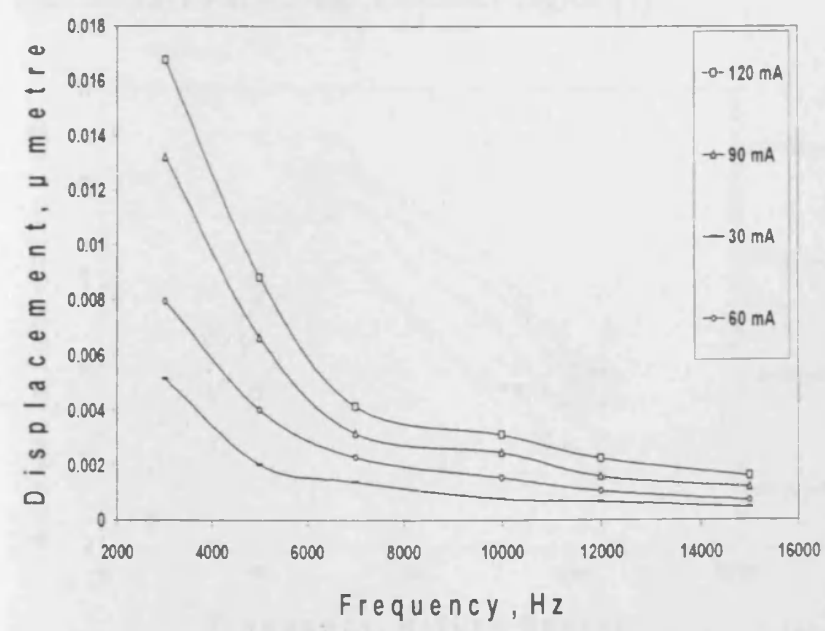

Fig. 5. High Frequency Response of a single transducer for various excitations current

For the moving magnet combination, frequency responses (Fig. 5 and 6) show gradual decrease in displacement as the frequency increases from low to high. Displacements recorded at various frequencies for different excitation currents show similar trends in displacements. This is very important since consistency in frequency response for various excitation currents is vital for loudspeaker application.

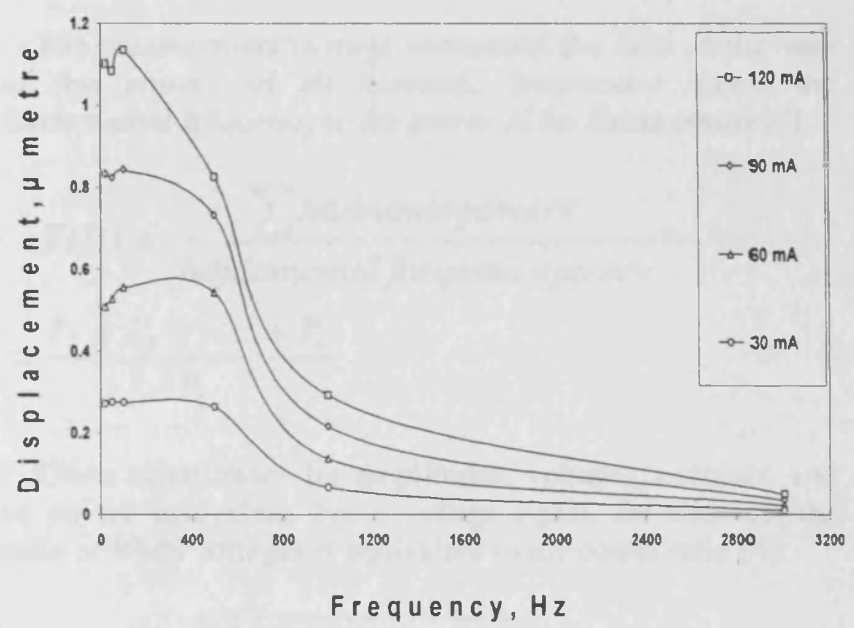

Fig. 6. High Frequency Response of a single transducer for various excitations current

\section{E. Resonance}

The magnitude of the displacement and the corresponding phase indicate that there are resonant peaks in the lowfrequency region. However, in the high-frequency region (> $2 \mathrm{kHz}$ ) no additional resonances were observed. Therefore, apart from the low-frequency end of the audio spectrum, the novel transducer speaker can reproduce a linear sound output across the whole audible frequency range.

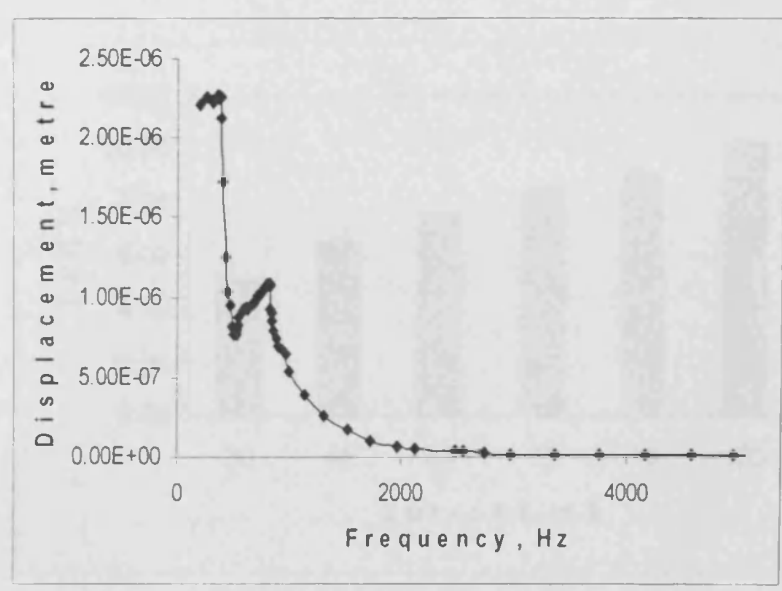


Figure 7: Magnitude curve showing resonance

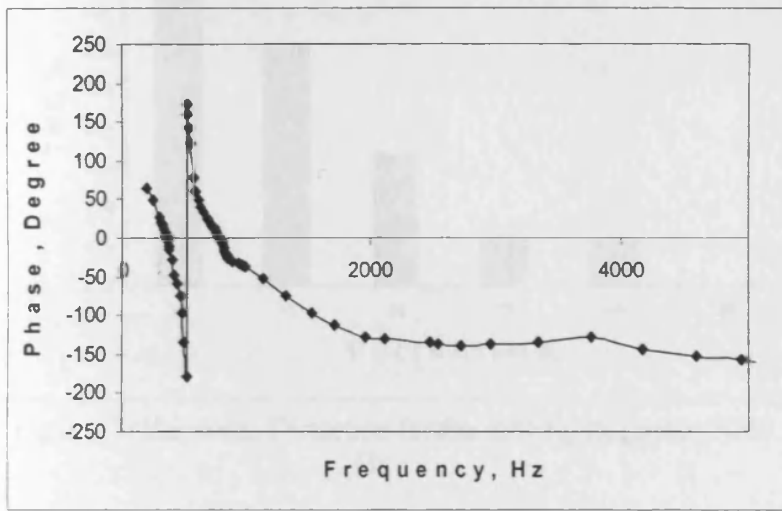

Figure 8: Phase curve showing resonance

\section{F. Acoustic Pressure Response}

The acoustic intensity curve shows gradual increase in intensity below $1 \mathrm{kHz}$ and gradual decrease in intensity above $1 \mathrm{kHz}$. Due to the small size, the acoustic intensity of the single transducer at the very low end of the audio spectrum (below $300 \mathrm{~Hz}$ ) is not adequate for human hearing. The low frequency limitation for a single transducer is evident from the actuator intensity levels (Fig. 11) since for a typical whole range audio loudspeaker, it is expected to have higher sound intensity at low frequencies and lower sound intensities at high frequencies. This is because the ear drum has a very low sensitivity at the low frequency region and high sensitivity at the high frequency region [1].

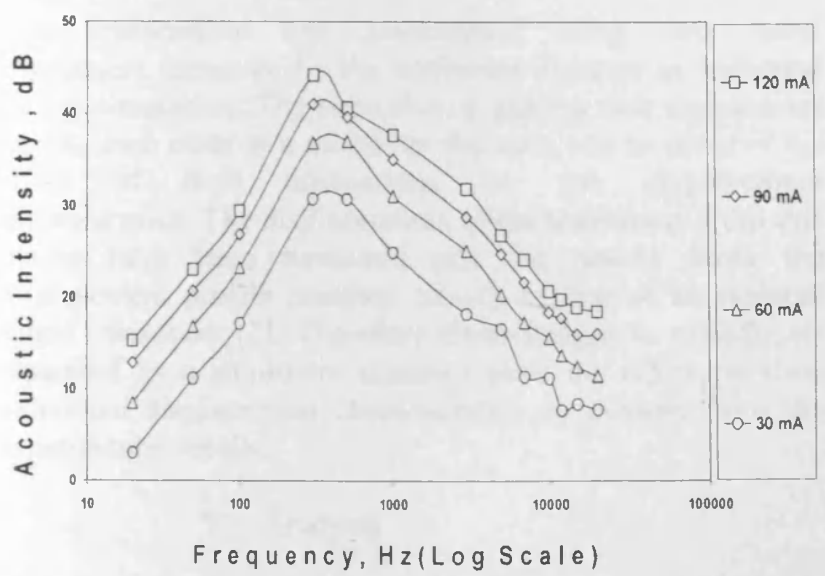

Figure 9: Acoustic intensity levels for the moving magnet combination

\section{G. Total Harmonic Distortion Measurement:}

The total harmonic distortion, or THD, of a signal is a measurement of the harmonic distortion present [4]. When a signal passes through a non-linear device, additional content is added at the harmonics of the original frequencies. This is a measurement of the extent of that distortion.

The measurement is most commonly the ratio of the sum of the powers of all harmonic frequencies above the fundamental frequency to the power of the fundamental [4]:

$$
\begin{aligned}
& T H D=\frac{\sum \text { harmonicpowers }}{\text { fundamental frequencypower }}= \\
= & \frac{P_{2}+P_{3}+\ldots \ldots+P_{n}}{P_{1}}
\end{aligned}
$$

Other calculations for amplitudes, voltages, currents, and so on are equivalent. For a voltage signal, for instance, the ratio of RMS voltages is equivalent to the power ratio [4]:

$$
T H D=\frac{\sqrt{V_{2}^{2}+V_{3}^{2}+\ldots \ldots . .+V_{n}^{2}}}{V_{1}}
$$

In this calculation, $V_{n}$ means the RMS voltage of harmonic $n$

The moving magnet configuration, as described before, is best suited for the loudspeaker system based on the frequency response, stability and intensity measurements. The following results (Fig. 12 and 13) at two different frequencies show the harmonic content for the moving magnet configuration at the low and range of the audio spectrum.

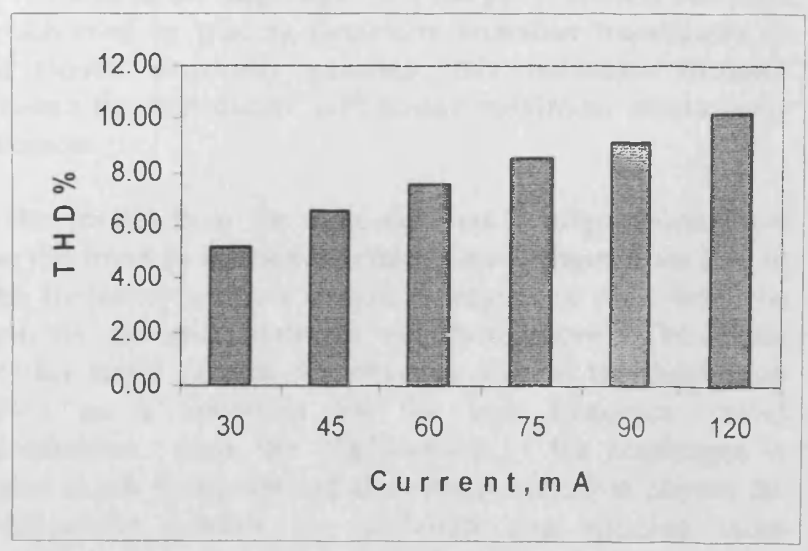

Figure 12: Harmonic Distortion for the moving magnet at $100 \mathrm{~Hz}$ 


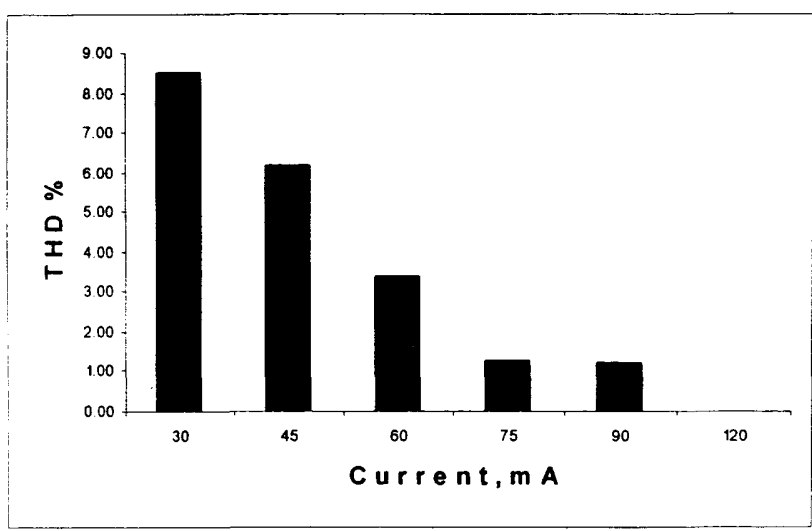

Figure 13: Harmonic Distortion for the moving magnet at 3000

$\mathrm{Hz}$

The harmonic distortion results show the amount of distortions in the reproduced sound. The single cell speaker shows two distinctive trends depending on the frequency range. At low frequency range, the THD increases with current but at high frequency, the THD decreases with current. Based on the overall THD results, the ideal operating current would be around $100 \mathrm{~mA}$. This exciting current level will ensure low THD and an efficient high frequency operation.

\section{Improvement of Bass Response}

Pressure responses from a $2 \times 2$ array and a $9 \times 2$ array matrix speaker have been measured using a high bandwidth microphone and the results were presented in a separate publication [2]. Acoustic pressure responses from the two different matrix arrays show the improvement in bass response for the larger array speaker.

\section{Interaction between transducers}

A loudspeaker was constructed using two novel transducers separated by the minimum distance as indicated by the simulation. The objective of placing two transducers next to each other at a minimum distance was to observe the effect of their interactions on the displacement characteristics. The displacements of the transducer from this setting have been measured and the results show the displacement profile matches closely to that of an isolated single transducer [3]. Therefore electromagnetic transducers separated by a minimum distance have no effect on their individual displacement characteristics as evident from the experimental results.

\section{Analysis}

The frequency responses of the moving magnet transducer show the linearity and consistent output of the transducer for various frequency and excitation currents. Also, the in terms of the mechanical structure of the moving magnet transducer, the lower amorphous panel and the magnet attract each other and hence give mechanical stability to the system. However, the acoustic intensity of the single transducer at the very low end of the audio spectrum (below $300 \mathrm{~Hz}$ ) is not adequate for human hearing. However, the low frequency hearing threshold has been improved (around $50 \mathrm{~Hz}$ ) significantly by using the same transducers in a matrix configuration [2].

Pressure responses from a $2 \times 2$ array and a 9x2 array matrix speaker have been measured using a high bandwidth microphone and the results were published elsewhere [2]. Both the speakers were driven with the same excitation current. For the $2 \times 2$ array speaker, the lowest frequency observed was $3 \mathrm{kHz}$ and below this frequency the sound pressure was too low to be measured by a high bandwidth microphone. For the $9 \times 2$ array speaker, however, the lowest frequency at which sound pressure could be measured was around $50 \mathrm{~Hz}$. The $9 \times 2$ array speaker has better acoustic pressure response in the bass region compared to the $2 \times 2$ array speaker at the same distance. The results from the pressure response measurement show the improvement in bass response for the $9 \times 2$ array speaker. A larger surface area and higher force obtained by employing more active transducers resulted in the improvement of the bass response for the longer array speaker.

Results predicted by FEM simulation has been reasonably agreed by experimental results. Minimum distance between transducers obtained by FEM simulation, to minimize the magnetic interaction have been implemented by placing two transducers next to each other and the results have proved that magnetic interactions and any effect on the individual displacement characteristics have been minimized. The transducers in the matrix array have been placed at a distance in which the repelling force matches the sum of the surface attraction force and the frictional force. At this 'minimum distance', the interaction between flux lines produced by the neighbouring transducers is not significant as confirmed by the simulation result. Since minimization of independent movements in the diaphragm of a flat panel speaker can only be achieved by placing numerous miniature transducers in the closest proximity possible, this minimum distance between the transducers will ensure maximum whole body vibration.

The results from the three different configurations show that the trend in harmonic distortions changes from low to high frequency. At low frequency region in each case, the harmonic distortion increases with current level. The reason for this could be that the physical size of the diaphragm serves as a limitation for the low frequency sound reproduction. Also, the displacement of the diaphragm is higher at low frequency and at a certain excitation current the displacement reaches its maximum and injecting more current results in higher harmonic distortion.

On the other hand, at high frequency, the limitation for diaphragm movement is the mass of the moving panel which is attached to the permanent magnet. The higher the load, the 
more force is needed to produce displacement and hence to get high acceleration from the panel more current is needed. Therefore at high frequency, harmonic distortion reduces with level of excitation current.

\section{Conclusion}

The matrix configuration of miniature transducers improves the low frequency response. The efficiency and sensitivity of miniature loudspeakers has been improved by means of loudspeakers with a large diaphragm area, high flux density, a high number of turns, a small voice coil resistance and small mass. The large diaphragm in a larger array speaker has extended the low frequency operation down to $50 \mathrm{~Hz}$. In a matrix array loudspeaker, electromagnetic transducers can operate without any mutual disturbance when separated by a minimum distance. The novel electromagnetic transducer can be implemented effectively for building matrix array speakers. Taking into consideration the consistent frequency response, improved bass response and minimized magnetic interaction the matrix array transducers can improve the existing loudspeaker technology.

\section{References}

1. J. M Eargle, Loudspeaker handbook, Kluwer Academic Publishers, $2^{\text {nd }}$ Edition, 2003

2. R. Rashedin, T. Meydan, F. Borza, "Electromagnetic Micro-actuator Array for Loudspeaker Application," Sensors and Actuators A: Physical, Vol.: 129, Issue: 1-2, 2006

3. R. Rashedin, T. Meydan, F. Borza," "A Novel Miniature Matrix Array Transducers System for Loudspeakers," IEEE Transactions on Magnetics, Vol.: 42, Issue: 10, 2006

4. O. Cugat, J. Delamare, G. Reyne, "Magnetic MicroActuators and Systems (MAGMAS)," IEEE Trans. on Magnetics, Vol.: 39, Issue: 6, 2003 


\title{
Harmonic Distortion Minimisation in Miniature Matrix Array Loudspeakers
}

\author{
R. Rashedin, T. Meydan*, and F. Borza \\ Wolfson Centre for Magnetics, School of Engineering, Cardiff University, Cardiff, CF243AA, UK
}

(Received: Xx Xxxx Xxxx. Accepted: Xx Xxxx Xxxx)

\begin{abstract}
A novel loudspeaker using miniature actuators in a matrix configuration has been designed to combine the advantages of conventional whole body motion with that of modern flat panel speakers. The proposed new loudspeaker was developed using these novel actuators in a matrix configuration. Measurements on single transducer have been carried out on three different device configurations in order to optimise the device. The three different configurations are: moving magnet, moving coil, and moving magnet with a plastic 'bottom layer' instead of the amorphous ribbon. Total harmonic distortions (THD) present in the three different device configurations were studied. At the low frequency region, typically in the range of $20 \mathrm{~Hz}$ to $500 \mathrm{~Hz}$, total harmonic distortion increases with current. At higher frequency, especially above $1 \mathrm{KHz}$, a current supply of $90 \mathrm{~mA}$ and over ensures high acceleration and hence a smooth high frequency response.
\end{abstract}

Keywords: Loudspeaker, Transducer, Total Harmonic Distortion.

\section{INTRODUCTION}

The novel loudspeaker having miniature transducers in a matrix array has been tested for three possible device configurations-Moving magnet, moving coil, and a moving magnet configuration with a plastic 'bottom layer' instead of an amorphous one. 'The results for the frequency response, sensitivity measurements and impedance have been explained' and the results indicate that the moving magnet configuration is best suited for loudspeaker application based on the low frequency response and mechanical stability. Now a new set of experiments for analyzing harmonic distortions in three different device configurations have been carried out. The results for the total harmonic distortion (THD) measurement show the variations in harmonic distortion for each configuration of the loudspeaker due to the change in frequency and excitation current.

\subsection{Total Harmonic Distortion}

The total harmonic distortion, or THD, of a signal is a measurement of the harmonic distortion present. ${ }^{2}$ When a signal passes through a non-linear devicc, additional content is added at the harmonics of the original

*Corresponding author: E-mail: meydan@cardiff.ac.uk frequencies. This is a measurement of the extent of that distortion.

The measurement is most commonly the ratio of the sum of the powers of all harmonic frequencies above the fundamental frequency to the pow'er of the fundamental:

$$
\begin{aligned}
\text { THD } & =\frac{\sum \text { Harmonic Powers }}{\text { Fundamental Frequency Power }} \\
& =\frac{P_{2}+P_{3}+\ldots \ldots+P_{n}}{P_{1}}
\end{aligned}
$$

Other calculations for amplitudes, voltages, currents, and so on are equivalent. For a voltage signal. for instance, the ratio of RMS voltages is equivalent to the power ratio: ${ }^{3}$

$$
\mathrm{THD}=\frac{\sqrt{V_{2}^{2}+V_{3}^{2}+\ldots \ldots \ldots+V_{n}^{2}}}{V_{1}}
$$

In this calculation, $V_{n}$ means the RMS voltage of harmonic $n$

\section{EXPERIMENTAL SET-UP}

The new transducer was tested in the audio frequency range by applying a sine wave current to the drive coil, with the resultant displacement of the front face measured using an OFV-303 laser vibrometer coupled to a digital lock-in amplifier (Fig. 1). The frequency was varied from 


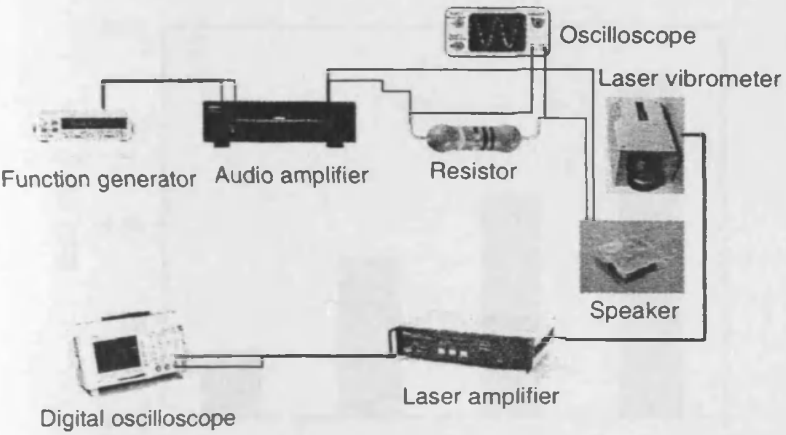

Fig. 1. Experimental set-up for displacement measurement.

$20 \mathrm{~Hz}$ to $20 \mathrm{kHz}$ with excitation currents levels from $30 \mathrm{~mA}$ to $120 \mathrm{~mA}$. The output voltages from the oscilloscope, which correspond to the displacements, were then analyzed for harmonic contents using the 'SIMPLORA' software. The software, using Fuurier analysis, derived the magnitudes of different harmonics present in the output voltage.

\subsection{Moving Magnet Configuration}

Based on the frequency response. mechanical stability, and intensity measurements, the moving magnet configuration was found to be best suited for matrix array loudspeaker system. ${ }^{1}$ The results for total harmonic distortion (Figs. 2 and 3) at two different frequencies show the harmonic content for the moving magnet configuration at the low and high range of the audio spectium.

\subsection{Moving Coil Configuration}

The moving coil configuration, as described in a previous paper. ${ }^{\prime}$ produces greater displacements at high frequencies but for low excitation currents it cannot produce any displacement at the low end of the audio spectrum. The harmonic distortion analysis for the moving coil configuration shows similar trends as the moving magnet arrangement. The following sets of results (Figs. 4 and 5) show the total harmonic distortions at low and high frequency regions.

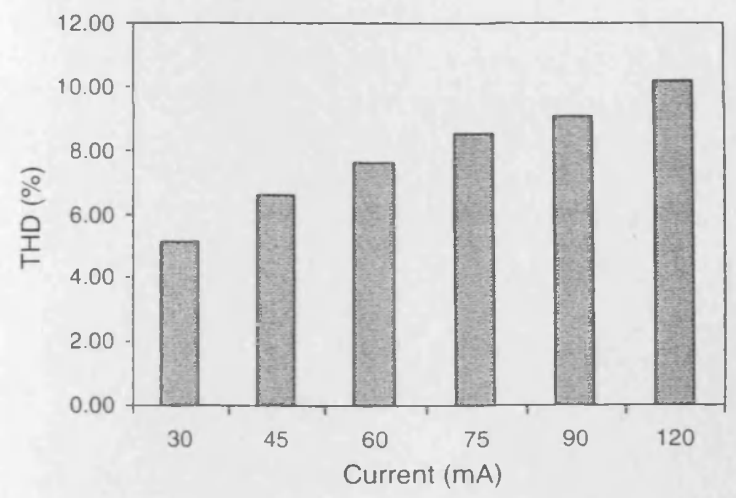

Fig. 2. Harmonic distortion for the moving magnet at $100 \mathrm{~Hz}$.

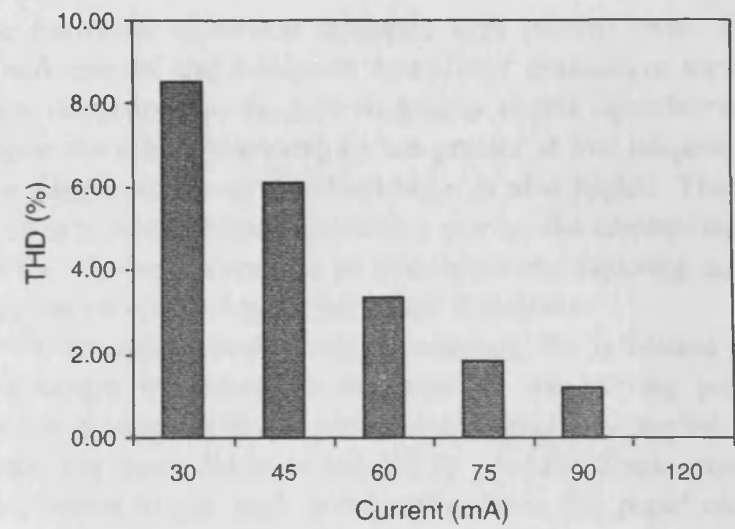

Fig. 3. Harmonic distortion for the moving magnet at $3000 \mathrm{~Hz}$

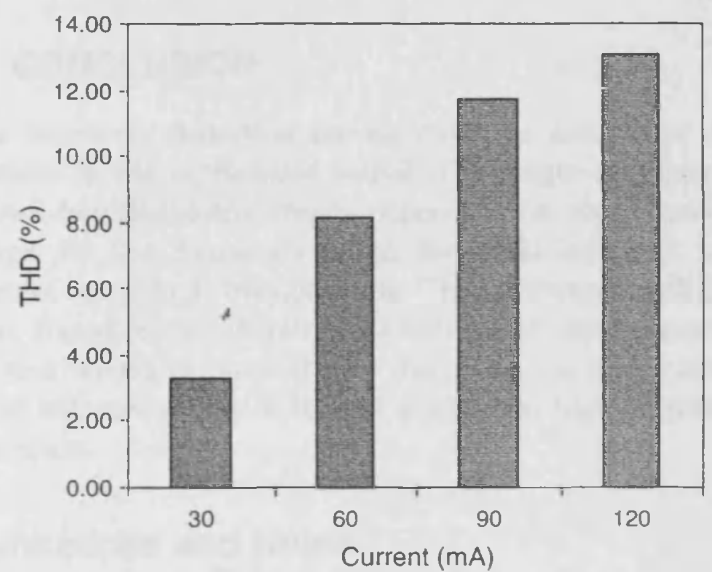

Fig. 4. Harmonic disturtion for the muving coil at $1000 \mathrm{~Hz}$.

\subsection{Moving Magnet with Plastic Bottom Laver}

The moving magnet configuartion. as described in a previous publicaion, ${ }^{1}$ is best suited for loudspeaker application. This moving magnet configuration with plastic "bottom layer' can also produce low frequency sound with low distortion. The following sets of results (Figs. 6 and 7) show the harmonic distortion levels for this configuration.

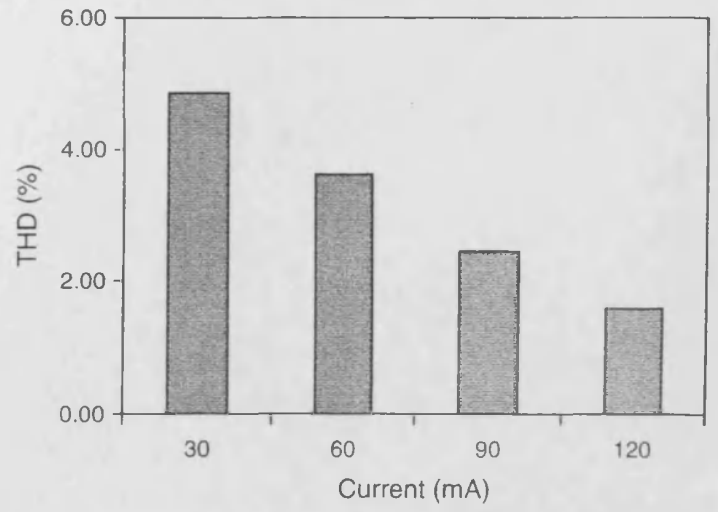

Fig. 5. Harmonic distortion for the moving coil at $3000 \mathrm{~Hz}$ 


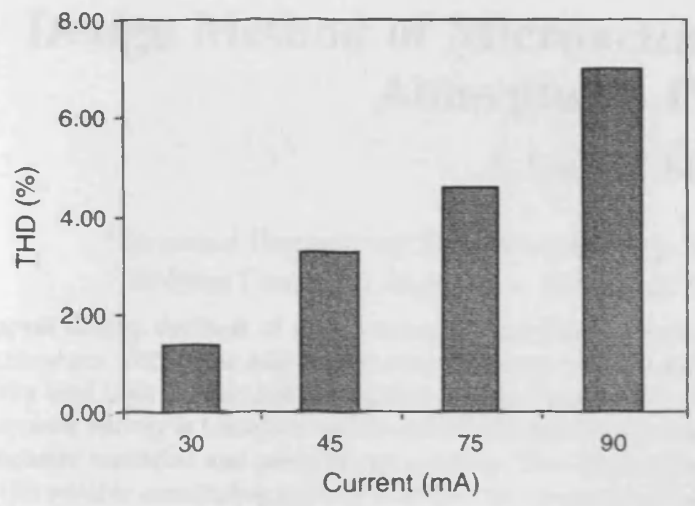

Fig. 6. Harmonic distortion for the moving magnet at $50 \mathrm{~Hz}$.

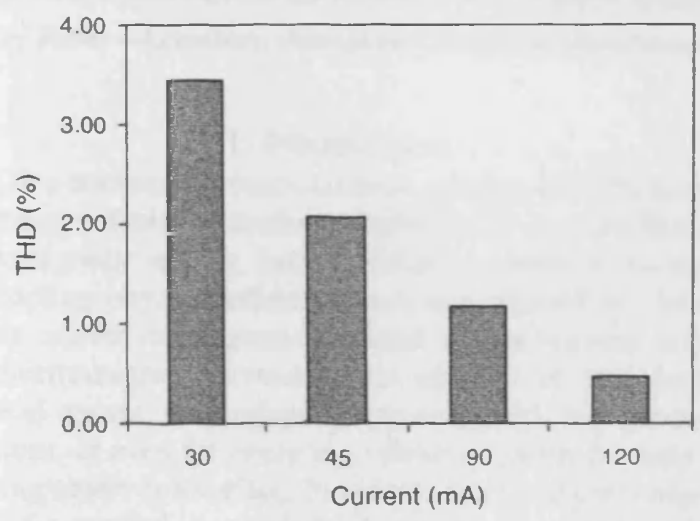

Fig. 7. Harmonic distortion for the moving magnet at $5000 \mathrm{~Hz}$.

\section{DISCUSSION}

The results from the three different configurations show that the trend in harmonic distortions changes from low to high frequency. At low frequency region in each case, the harmonic distortion increases with current level. The small size of the miniature transducer diaphragm serves as a limitation for the low frequency sound reproduction. Since the sound wavelengths are greater at low frequency, the displacement of the diaphragm is also higher. Therefore at a certain higher excitation current the displacement of the diaphragm reaches its maximum and injecting more current results in higher harmonic distortion.

On the other hand, at high frequency, the limitation for diaphragm movement is the mass of the moving panel which is attached to the permanent magnet. The higher the load, the more force is needed to produce displacement and hence to get high acceleration from the panel more current is needed. Therefore at high frequency, harmonic distortion reduces with level of excitation current.

\section{CONCLUSION}

The harmonic distortion results show the amount of distortions in the reproduced sound. The single cell speaker shows two distinctive trends depending on the frequency range. At low frequency range, the THD increases with current but at high frequency, the THD decreases with current. Based on the overall THD results, the ideal operating current would be around $100 \mathrm{~mA}$. This exciting current level will ensure low THD and an efficient high frequency operation.

\section{References and Notes}

1. R. Rashedin, T. Meydan, and Borza, IEEE Trans. Magn. (2006), in press

2. Total Harmonic Distortion, Wikipedia; http://en.wikipedia.org/ wiki/Total harmonic_distortion.

3. Shmilovit7. DIEEE Transactions on Power Delivery 20, 526 (2005). 


\title{
Design Method of Microactuator With Magnetic Alloy Iron-Based Amorphous Plates for Loudspeaker
}

\author{
T. Lin ${ }^{1}$, T. Meydan ${ }^{2}$, and R. Rashedin ${ }^{2}$ \\ ${ }^{1}$ Electrical Engineering Department, Yung-Ta Institute of Technology and Commerce, Taiwan, R.O.C. \\ ${ }^{2}$ Wolfson Centre for Magnetics, School of Engineering, Cardiff University, Cardiff, CF24 3AA, U.K.
}

A novel design method of microactuator has been developed and results show that this transducer could produce sinusoidal wave displacements within the audible frequency range for the miniature electromagnetic loudspeaker. The proposed actuator has high conductivity and high permeability magnetic alloy iron-based amorphous plates as diaphragms. In this novel transducer speaker, the electromac netic energy is transformed to mechanical pressure waves as a result of the coupling physical effects between time varying current on magnetic material and permanent magnet. The prototype actuator, based on the proposed model, has been characterized using the laser vibrometer measuring system to detect the amplitudes of displacement at all octave bands. This new miniature transducer speaker aims to provide a solution to the miniaturization of loudspeaker with flat panel speaker technology, which operates under the bending wave principle, and exploits the resonance of the panel instead of the traditional piston motion to produce sound.

Index Terms-Actuators, amorphous materials, electroacoustic transducers, electromagnetic forces.

\section{INRODUCTION}

$\mathbf{F}$ OR a traditional voice-coil cone speaker, the fundamental theory of piston motion diaphragm is to transform the electromagnetic energy into mechanical pressure waves by the coupling physical effects, which are induced by the time varying current on magnetic material and permanent magnet. The electromagnetic transducer is capable of transforming electrical power to mechanical power, used for producing vibrations, or even for canceling vibrations, with the help of a vibrating object or a surface. In order to have sufficient expanse or area for producing sound, the diaphragm in a loudspeaker is usually designed as a shaped cone connected with the moving coil which is located at the gap of magnetic circuit and thus obtaining orthogonal magnetic flux to produce mechanical resonance and longitudinal acoustic waveforms [1]-[4]. The performance of the dynamic cone drivers are distorted by nonlin ar mechanical characteristics and the driving forces, which ire from assembling parts and nonuniform magnetic flux distribution on magnetic materials [5]. At present, the development of panel speaker technology, different from the earlier approaches, concentrates mainly on less space, less directional sound. and preferably less weight by using loudspeaker configuration employing thin panels. The approach involves use of materials capable of sustaining bending waves and generating sound from action of those bending waves [5]-[13]

The voltage equation [2] for the magnetic circuit of the proposed transducer shows that if the $B_{g} l$ is constant in (1), the active mechanical pressure on the diaphragm in voice-coil type will be proportional to $i$, and the distortion will arise due to the nonuniform magnetic flux distribution in the moving voice coil; meanwhile, the time varying vibrating distance of voice-coil will also be affected by $B_{g}$

$$
e=R i+L \frac{d i}{d t}+B_{g} l \frac{d x}{d t}
$$

Digital Object Identifier 10.1109/TMAG.2007.893780

Color versions of one or more of the figures in this paper are available online at http:/ eeexplore.ieee.org. where

$$
\begin{array}{ll}
e & =\text { electric sources; } \\
R & =\text { resistance; } \\
L & =\text { inductance of voice-coil; } \\
i & =\text { exciting currents of voice-coil; } \\
B_{g} & =\text { magnetic flux density at air gap; } \\
l & =\text { the effective distance of voice-coil; } \\
x & =\text { vibrating distance of voice-coil. }
\end{array}
$$

Sound does not normally consist of single-frequency tone, but a highly complex combination of tones and, therefore, it is necessary to know the bands of frequencies present in a sound spectrum. In most cases, it is sufficient to know the magnitude of the sound waves contained within the octave bands: 75-150, $150-300,300-600,600-1200,1200-2400,2400-4800$, and $4800-9600 \mathrm{~Hz}$. It can be seen that one octave band consists of all sounds from any frequency to twice that same frequency. In each case, it is convenient to refer only to the center frequency within each band; e.g., 125, 250, 500, $1 \mathrm{k}, 2 \mathrm{k}, 4 \mathrm{k}, 8 \mathrm{k} \mathrm{Hz}$ for octave [1]. In order to observe the property of the magnetic alloy iron-based amorphous material which is used as the panel for the proposed novel flat panel speaker, the displacements of the thin amorphous panel was used as an index parameter to evaluate the velocity bending waves. Velocity bending waves $V_{b}$, given by (2), are dependent within the designed frequencies, e.g., 16, 31.5, 63, 125, 250, 500, 1000, 2000, 4000, 8000, 10k $20 \mathrm{k} \mathrm{Hz}$ for octave [1] on the amorphous plate

$$
V b=\left[\frac{E I}{A \rho}\right]^{1 / 4} \cdot \omega^{1 / 2}
$$

where

$$
\begin{aligned}
& E=\text { Young's modulus of elasticity; } \\
& I=\text { second moment of area; } \\
& A=\text { cross-sectional area of plate; } \\
& \rho=\text { density; } \\
& \omega \quad=\text { exciting frequency in radians/s. }
\end{aligned}
$$




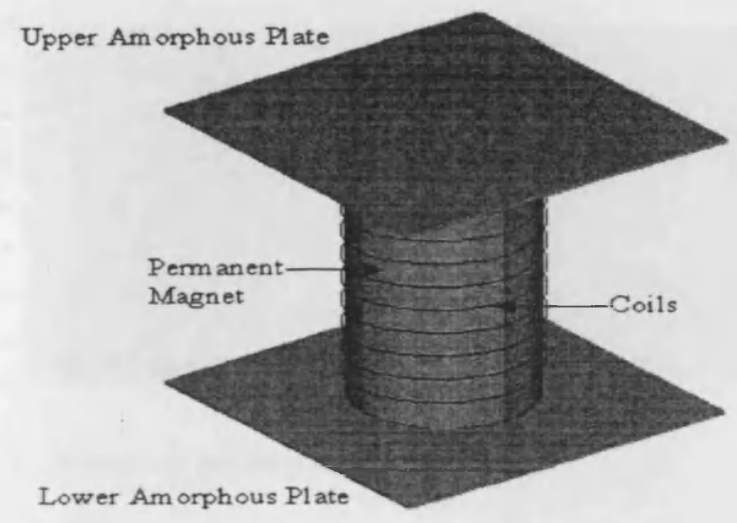

Fig. 1. The 3-D FEM magnetic model.

\section{Physical Model Description}

According to the proposed physical model, the magnetic force on the amorphous plates that develops in the microactuator depends on the magnetic field and the current in the coils. When the magnetic field is constant, the magnetic force depends only on the current density distribution. As a time-varying excitation current induces a varying magnetic field, the field induces currents in the neighboring magnetic materials. This phenomenon is illustrated by a time-varying field simulation as well as electromagnetic quasi-static analysis. In the physical model, the current-carrying coils are placed between the two amorphous plates. The coils are surrounded by air, and there is a small air gap between the coils and amorphous plates. The total current density in the coils is obtained by taking the induced currents into account. The Lorentz force on the plate caused by the eddy currents can also be computed by the finite-element method (FEM) using this magnetic circuit model. The 3-D FEM magnetic model is shown in Fig. 1 and the magnetic flux density distribution, as indicated by the streamlines, is shown in Fig. 2.

The physical model includes of a 3-mm diameter and 2-mm length $\mathrm{N} 30 \mathrm{H}$ disc which is a sintered neodymium-iron-boron perma ient magnet axially magnetized through thickness with remence $B_{r}=11.2 \mathrm{kG}$ and maximum energy product $(B H)_{\max }=30$, coils, and $36-\mathrm{mm}$ square panels of 20 - $\mu$ m-thick magnetic alloy iron-based amorphous plate.

The displacements from this transducer have been obtained by using the laser vibrometer measuring system, as shown in Fig. 3. The motion of the top diaphragm was measured by the laser vibrometer (model Polytec OFV 303 Signal Head), and the signal head was controlled by a Polytec OFV 3001 vibrometer controller. The noncontact laser vibration measurement system has displacement resolution down to $0.002 \mu \mathrm{m}$. The output from the laser vibrometer was processed using a digital oscilloscope. The experimental results (Figs. 4-6) were marginally affected by the inherent low-frequency component present in the vibration system and the measuring environment.

\section{EXPERIMENTAL INVESTIGATIONS AND CONCLUSION}

In the experiments, the magneto-mechanical resonant responses have been obtained at $16 \mathrm{~Hz}, 31.5 \mathrm{~Hz}, 63 \mathrm{~Hz}, 125 \mathrm{~Hz}$, $250 \mathrm{~Hz}, 500 \mathrm{~Hz}, 1 \mathrm{kHz}, 2 \mathrm{kHz}, 4 \mathrm{kHz}, 8 \mathrm{kHz}, 10 \mathrm{kHz}$, and

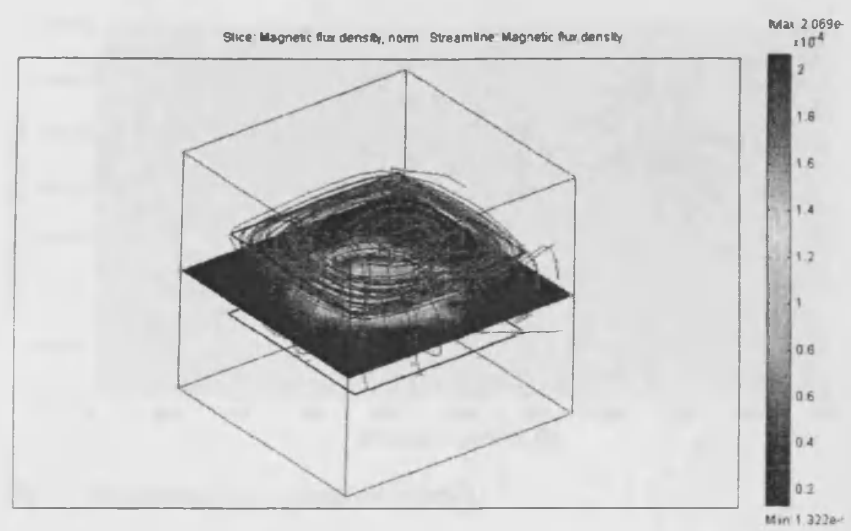

Fig. 2. FEM magnetic flux density streamline distribution.

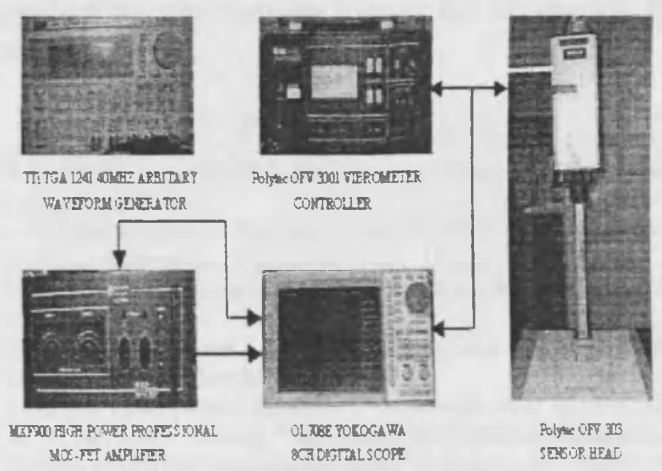

Fig. 3. Laser vibrometer measuring system.

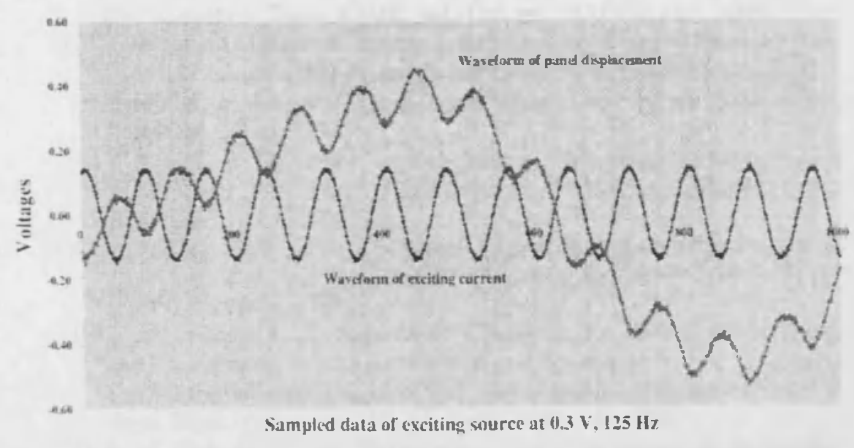

Fig. 4. Displacement measuring waveforms at $125 \mathrm{~Hz}$.

at $20 \mathrm{kHz}$. At each frequency, the experiment was conducted using an excitation voltage of $0.3 \mathrm{~V}$. From the experimental results, the magneto-mechanical resonances are apparent at low frequencies. For the prototype model of the proposed loudspeaker, Figs. 4-6 showed the comparison between the waveforms of the time-varying excitation currents and plate displacements at $125 \mathrm{~Hz}, 500 \mathrm{~Hz}$, and $2 \mathrm{kHz}$, respectively. The magnitudes of the displacements obtained are from 0.795 to $0.011 \mu \mathrm{m}$, respectively, from $16 \mathrm{~Hz}$ to $20 \mathrm{kHz}$. These characteristics can be applied to improve the low frequency response of a flat panel speaker using the proposed model, and the characteristics of bending waves can be also estimated clearly. The experimental results show that the proposed model can produce 


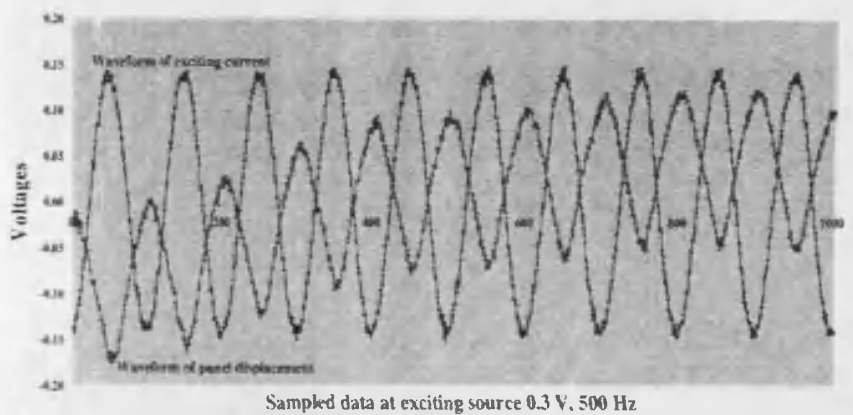

Fig. 5. Displacement measuring waveforms at $500 \mathrm{~Hz}$.

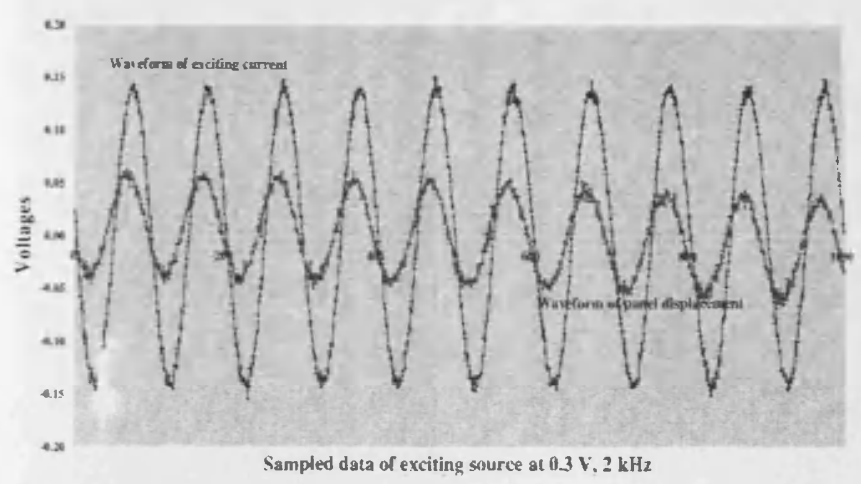

Fig. 6. Displacement measuring waveforms at $2 \mathrm{kHz}$.

an active and real energy source and can engage the mechanical pressure with bending waves on the high conductivity and high permeability magnetic alloy iron-based thin amorphous plates. Also the magneto-mechanical resonant responses have been obtained for excitation voltages of $0.3,0.35,0.4$, and $0.5 \mathrm{~V}$. For the prototype model of the proposed loudspeaker, the displacement profile showed an exponential decrease from low to high frequency, as shown in Fig. 7.

In this paper, the novel design method of the miniature actuator using magnetic energy provides a potential solution of miniaturization for the flat panel speakers. This novel miniature actuator having thin amorphous ribbons as diaphragms is demonstrated to improve at low fundamental frequency with high quality acoustic output for flat panel speaker technology.

\section{ACKNOWLEDGMENT}

This work was supported in part by Wolfson Centre for Magnetics, School of Engineering, Cardiff University, U.K. The work was initiated at Wolfson Centre for Magnetics, School

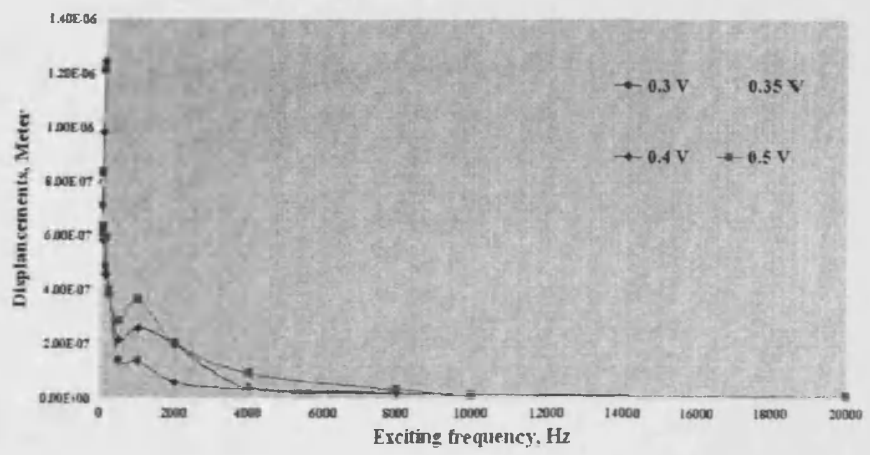

Fig. 7. Measuring displacement distribution.

in Engineering, Cardiff University, U.K. The work of T. Lin was supported by the Wolfson Centre for Magnetics, Cardiff University.

\section{REFERENCES}

[1] B. J. Smith, ACOUSTICS- Environment Physics. White Plains, NY: Longman, 1971.

[2] J. D. Turner and A. J. Pretlove, ACOUSTICS for ENGINEERS. Basingstoke, Hampshire: Macmilan, 1991, Houdmills

[3] M. L. Gayford, Electroacoustics. London: Newnes-Butter Worths, 1970.

[4] R. W. B. Stephens and A. E. Bate, Acoustics and Vibrational Pyhsics. London: Edward Arnold Ltd., 1966

[5] J. Eargle, Loudspeaker Handbook. Norwell, MA: Kluwer, 2003

[6] M. R. Bai and T. Huang, "Development of panel loudspeaker system: Design, evaluation and enhancement,", J. Acoust. Soc. Amer., vol. 109. no. 6 , pp. 2751-2761, Jun. 2001

[7] M. R. Baiand and J. Kuo, "Optimal design and implementation of an omnidirectional panel speaker array using the genetic algorithm." $J$. Vib. Acoustics, Trans. ASME, vol. 126, pp. 553-560, Oct. 2004.

[8] C. Divoux, O. Cugat, G. Reyne, J. Boussey-Sai'd. and S. Basrour. "Deformable mirror using magnetic membranes: Application to adaptive optics in astrophysics," IEEE Trans. Magn., vol. 34, no. 5, pt. 1. pp. 3564-3567, Sep. 1998.

[9] O. Cugat, J. Delamare, and G. Reyne, "Magnetic micro-actuators and systems (MAGMAS)," IEEE Trans. on Mag., vol. 39, no. 6, pp. 3607-3612, Nov. 2003.

[10] M. R. Bai and K. Chung, "Optimal design of panel speaker array with omnidirectional characteristics," J. Acoust. Soc. Amer., vol. 112, pp. 1944-1952, Nov. 2002.

[11] G. Y. Hwang, K. T. Kim, S. U. Chung, S. M. Hwang, B. S. Kang, and I. C. Hwang, "Analysis of a dynamic speaker in mobile phones by considering mechanical, electrical, and magnetic coupling effects.," $j$. Appl. Phys., vol. 91, no. 10, pp. 6979-6981, May 2002.

[12] R. M. Aarts and A. J. E. M. Janssen, "On analytic design of loudspeaker arrays with uniform characteristics.," J. Acoust. Soc. Amer., vol. 107. no. 1, pp. 287-292, Jan. 2000.

[13] C. K. Campbell, Surface Acoustic Wave Devices. New York: Academic, 1998.

Manuscript received October 29,2006 (e-mail: tklin@mail.ytit.edu.tw).

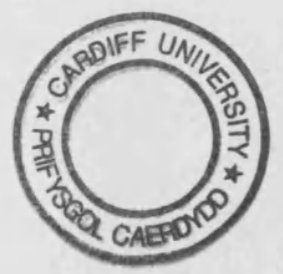

